\title{
Stereodivergent Synthesis of $\alpha$-Quaternary Serine and Cysteine Derivatives Containing Two Contiguous Stereogenic Centers via Synergistic Cu/Ir Catalysis
}

\footnotetext{
Hui-Min Wu, ${ }^{\mathrm{a},}$ Zongpeng Zhang, ${ }^{\mathrm{a}}{ }^{\S}$ Fan Xiao, ${ }^{\mathrm{a}}$ Liang Wei, ${ }^{\mathrm{a}}{ }^{\mathrm{X}}$ Xiu-Qin Dong, ${ }^{* a}$ and Chun-Jiang Wang*a,b

a Engineering Research Center of Organosilicon Compounds \& Materials, Ministry of Education, College of Chemistry and Molecular Sciences, Wuhan University, Wuhan, 430072, China

${ }^{\mathrm{b}}$ State Key Laboratory of Elemento-organic Chemistry, Nankai University, Tianjin, 300071, China
}

\section{Contents}

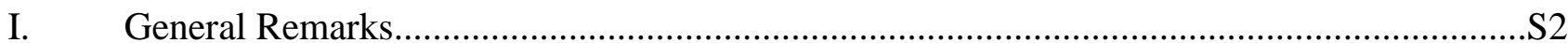

II. General Procedure for Dual $\mathrm{Cu} / \mathrm{Ir}$ Catalyzed Allylic Alkylation Reaction of Cyclic Imine Esters

III. Gram Scale Reaction and Synthetic Transformation

IV. Absolute Configuration Determination of $(S, S)-3$ he

V. Reference S23

VI. $\quad{ }^{1} \mathrm{H}$ NMR and ${ }^{13} \mathrm{C}$ NMR Spectra. S24

VII. HPLC Spectra. S68 


\section{General Remarks}

${ }^{1} \mathrm{H}$ NMR spectra were recorded on a Bruker $400 \mathrm{MHz}$ spectrometer in $\mathrm{CDCl}_{3}$. Chemical shifts are reported in ppm with the internal TMS signal at $0.0 \mathrm{ppm}$ as a standard. The data are reported as (s $=$ single, $\mathrm{d}=$ double, $\mathrm{t}=$ triple, $\mathrm{q}=$ quarte, $\mathrm{m}=$ multiple or unresolved, brs $=$ broad single, coupling constant(s) in $\mathrm{Hz}$, integration). ${ }^{13} \mathrm{C}$ NMR spectra were recorded on a Bruker $100 \mathrm{MHz}$ spectrometer in $\mathrm{CDCl}_{3}$. Chemical shifts are reported in ppm with the internal chloroform signal at $77.0 \mathrm{ppm}$ as a standard. Commercially obtained reagents were used without further purification. Solvents were purified prior to use according to the standard methods. Unless otherwise noted, all reactions were carried out under nitrogen atmosphere. All reactions were monitored by TLC with silica gel coated plates. Enantiomeric ratios were determined by chiral-phase HPLC analysis in comparison with authentic racemic materials using a chiralpak AD-H, OD-H and IE column with hexane and $i-\mathrm{PrOH}$ as solvents. High Resolution Mass Spectra were recorded using ESI-TOF technique. 2-Oxazoline and 2-thiazoline-4-carboxylate, ${ }^{1}$ allylcarbonates, ${ }^{2} \mathbf{L 1 - L 4}{ }^{3}$ and $\mathbf{L 5}^{4}$ were prepared according to the literature procedure. The absolute configuration of 3he was determined by X-ray analysis, and those of other adducts were deduced on the basis of this result. The racemic products were obtained by running reactions with a racemic catalyst or blending equal amount of two enantiomers.

\section{General Procedure for Dual Cu/Ir Catalyzed Allylic Alkylation Reaction of Cyclic Imine} Esters

A flame dried Schlenk tube was cooled to $\mathrm{rt}$ and filled with $\mathrm{N}_{2}$. To this flask were added $[\operatorname{Ir}(\mathrm{COD}) \mathrm{Cl}]_{2}(0.0025 \mathrm{mmol}, 1.25 \mathrm{~mol} \%)$, phosphoramidite ligand $(S, S, S)-\mathbf{L 5}(0.005 \mathrm{mmol}, 2.5$ mol \%), degassed THF $(0.5 \mathrm{~mL})$ and degassed $n$-propylamine $(0.5 \mathrm{~mL})$. The reaction mixture was heated at $50{ }^{\circ} \mathrm{C}$ in oil bath for $30 \mathrm{~min}$ and then the volatile solvents were removed under vacuum to give a pale-yellow solid. Meanwhile, in a separated Schlenk tube, $\left(R, R_{p}\right){ }^{i}{ }^{i}$ Pr-Phosferrox-L1 $(0.011$ mmol, $5.5 \mathrm{~mol} \%)$ and $\mathrm{Cu}(\mathrm{MeCN})_{4} \mathrm{BF}_{4}(0.01 \mathrm{mmol}, 5 \mathrm{~mol} \%)$ were dissolved in $1.0 \mathrm{~mL}$ of $\mathrm{THF}$, and stirred at room temperature for about $0.5 \mathrm{~h}$. The $\mathrm{Cu}(\mathrm{I}) / \mathbf{L} \mathbf{1}$ complex solution was then transformed to the Schlenk tube containing iridium complex via syringe. Allylic carbonates $(0.30 \mathrm{mmol})$, 2-oxazoline or 2-thiazoline-4-carboxylate $(0.20 \mathrm{mmol}), \mathrm{Et}_{3} \mathrm{~N}(0.20 \mathrm{mmol})$, THF $(1.0 \mathrm{~mL})$ were then added. Once starting material was consumed, the organic solvent was removed by rotary evaporation. 
The dr value was determined by ${ }^{1} \mathrm{H}$ NMR analysis of the crude mixture and the residue was purified by column chromatography to give the allylation product, which was then directly analyzed by HPLC to determine the enantiomeric excess.

\section{Methyl (S)-2-phenyl-4-((S)-1-phenylallyl)-4,5-dihydrooxazole-4-carboxylate (3aa):}<smiles>C=CC(c1ccccc1)[C@@]1(C(C)=O)COC(c2ccccc2)=N1</smiles>

Yield (70\%); $45.0 \mathrm{mg}$; yellow oil; it was purified by column chromatography (silica gel, eluent: petroleum ether: ethyl acetate $=10: 1) .[\alpha]^{20} \mathrm{D}=+167.7\left(c 1.1, \mathrm{CHCl}_{3}\right) ;{ }^{1} \mathrm{H} \mathrm{NMR}\left(400 \mathrm{MHz}, \mathrm{CDCl}_{3}\right) \delta$ $7.99-7.90(\mathrm{~m}, 2 \mathrm{H}), 7.52-7.45(\mathrm{~m}, 1 \mathrm{H}), 7.42-7.37(\mathrm{~m}, 2 \mathrm{H}), 7.25-7.16(\mathrm{~m}, 5 \mathrm{H}), 6.34-6.25(\mathrm{~m}, 1 \mathrm{H})$, 5.20-5.11 (m, 2H), $4.71(\mathrm{~d}, J=9.2 \mathrm{~Hz}, 1 \mathrm{H}), 4.49(\mathrm{~d}, J=9.2 \mathrm{~Hz}, 1 \mathrm{H}), 4.05(\mathrm{~d}, J=8.4 \mathrm{~Hz}, 1 \mathrm{H}), 3.71$ (s, 3H). ${ }^{13} \mathrm{C}$ NMR $\left(100 \mathrm{MHz}, \mathrm{CDCl}_{3}\right) \delta 172.9,165.3,137.9,136.0,131.7,129.2,128.5,128.4,128.2$, 127.3, 127.0, 118.5, 82.1, 71.7, 56.4, 52.5. HRMS (ESI-TOF) Calcd. For $\mathrm{C}_{20} \mathrm{H}_{19} \mathrm{NO}_{3}\left([\mathrm{M}+\mathrm{H}]^{+}\right)$: 322.1438, found: 322.1436. The product was analyzed by HPLC to determine the enantiomeric excess: $>99 \%$ ee (Chiralpak AD-H, $i$-propanol/hexane $=5 / 95$, flow rate $1.0 \mathrm{~mL} / \mathrm{min}, \lambda=254 \mathrm{~nm})$; $\mathrm{t}_{\mathrm{r}}$ $=6.13$ and $10.57 \mathrm{~min}$.

\section{Methyl (S)-2-(4-(tert-butyl) phenyl)-4-((S)-1-phenylallyl)-4,5-dihydrooxazole-4-carboxylate} (3ba):

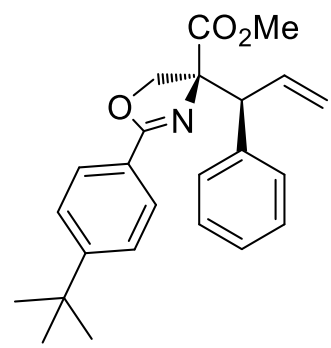

Yield (40\%); $30.2 \mathrm{mg}$; yellow oil; it was purified by column chromatography (silica gel, eluent: petroleum ether: ethyl acetate $=25: 1-10: 1) .[\alpha]^{20} \mathrm{D}=+154.5\left(c 1.0, \mathrm{CHCl}_{3}\right) ;{ }^{1} \mathrm{H}_{\mathrm{NMR}}(400 \mathrm{MHz}$, $\left.\mathrm{CDCl}_{3}\right) \delta 7.91-7.88(\mathrm{~m}, 2 \mathrm{H}), 7.42(\mathrm{~d}, J=8.7 \mathrm{~Hz}, 2 \mathrm{H}), 7.27-7.17(\mathrm{~m}, 5 \mathrm{H}), 6.30-6.23(\mathrm{~m}, 1 \mathrm{H})$, 5.20-5.11 (m, 2H), $4.72(\mathrm{~d}, J=9.1 \mathrm{~Hz}, 1 \mathrm{H}), 4.47(\mathrm{~d}, J=9.1 \mathrm{~Hz}, 1 \mathrm{H}), 4.07(\mathrm{~d}, J=8.4 \mathrm{~Hz}, 1 \mathrm{H}), 3.70$ 
(s, 3H), $1.33(\mathrm{~s}, 9 \mathrm{H}) .{ }^{13} \mathrm{C} \mathrm{NMR}\left(100 \mathrm{MHz}, \mathrm{CDCl}_{3}\right) \delta 173.0,165.3,155.2,138.0,136.0,129.1,128.39$, 128.35, 127.3, 125.2, 124.1, 118.5, 82.0, 71.5, 56.2, 52.5, 34.9, 31.1. HRMS (ESI-TOF) Calcd. For $\mathrm{C}_{24} \mathrm{H}_{27} \mathrm{NO}_{3}\left([\mathrm{M}+\mathrm{H}]^{+}\right): 378.2064$, found: 378.2056 . The product was analyzed by HPLC to determine the enantiomeric excess: $>99 \%$ ee (Chiralpak AD-H, $i$-propanol $/$ hexane $=5 / 95$, flow rate $1.0 \mathrm{~mL} / \mathrm{min}$, $\lambda=254 \mathrm{~nm}) ; \mathrm{t}_{\mathrm{r}}=5.32$ and $10.62 \mathrm{~min}$.

Methyl (S)-2-(4-methoxyphenyl)-4(S)-(1-phenylallyl)-4,5-dihydrooxazole-4-carboxylate (3ca):<smiles>C=CC(c1ccccc1)C(C)(C)C1=NC(c2ccc(OC)cc2)=NC1</smiles>

Yield (63\%); $44.2 \mathrm{mg}$; yellow oil; it was purified by column chromatography (silica gel, eluent: petroleum ether: ethyl acetate $=10: 1) .[\alpha]^{20} \mathrm{D}=+185.8\left(c 1.0, \mathrm{CHCl}_{3}\right) ;{ }^{1} \mathrm{H} \mathrm{NMR}\left(400 \mathrm{MHz}, \mathrm{CDCl}_{3}\right) \delta$ $7.89(\mathrm{~d}, J=8.8 \mathrm{~Hz}, 2 \mathrm{H}), 7.26-7.16(\mathrm{~m}, 5 \mathrm{H}), 6.89(\mathrm{~d}, J=8.8 \mathrm{~Hz}, 2 \mathrm{H}), 6.33-6.24(\mathrm{~m}, 1 \mathrm{H}), 5.19-5.09$ (m, 2H), $4.66(\mathrm{~d}, J=9.1 \mathrm{~Hz}, 1 \mathrm{H}), 4.45(\mathrm{~d}, J=9.1 \mathrm{~Hz}, 1 \mathrm{H}), 4.02(\mathrm{~d}, J=8.4 \mathrm{~Hz}, 1 \mathrm{H}), 3.83(\mathrm{~s}, 3 \mathrm{H})$, 3.70 (s, 3H). ${ }^{13} \mathrm{C}$ NMR $\left(100 \mathrm{MHz}, \mathrm{CDCl}_{3}\right) \delta 173.1,165.1,162.3,137.9,136.1,130.3,129.2,128.3$, 127.2, 119.4, 118.4, 113.5, 82.0, 71.6, 56.4, 55.3, 52.5. HRMS (ESI-TOF) Calcd. For $\mathrm{C}_{21} \mathrm{H}_{21} \mathrm{NO}_{4}$ $\left([\mathrm{M}+\mathrm{H}]^{+}\right): 352.1543$, found: 352.1538 The product was analyzed by HPLC to determine the enantiomeric excess: $>99 \%$ ee (Chiralpak AD-H, $i$-propanol/hexane $=5 / 95$, flow rate $1.0 \mathrm{~mL} / \mathrm{min}, \lambda$ $=254 \mathrm{~nm}) ; \mathrm{t}_{\mathrm{r}}=8.74$ and $22.45 \mathrm{~min}$.

Methyl (S)-4-((S)-1-phenylallyl)-2-(3,4,5-trimethoxyphenyl)-4,5-dihydrooxazole-4-carboxylate (3da):

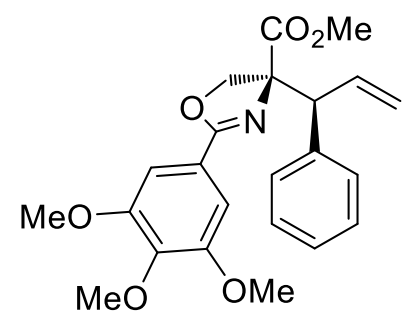

Yield (54\%); $44.4 \mathrm{mg}$; yellow oil; it was purified by column chromatography (silica gel, eluent: petroleum ether: ethyl acetate $=10: 1-8: 1) .[\alpha]^{20} \mathrm{D}=+310.7\left(c 0.6, \mathrm{CHCl}_{3}\right) ;{ }^{1} \mathrm{H} \mathrm{NMR}(400 \mathrm{MHz}$, 
$\left.\mathrm{CDCl}_{3}\right) \delta$ 7.29-7.20 (m, 7H), 6.31-6.22 (m, 1H), 5.23-5.11 (m, 2H), $4.72(\mathrm{~d}, J=8.8 \mathrm{~Hz}, 1 \mathrm{H}), 4.49(\mathrm{~d}$, $J=9.1 \mathrm{~Hz}, 1 \mathrm{H}), 4.08(\mathrm{~d}, J=8.4 \mathrm{~Hz}, 1 \mathrm{H}), 3.91(\mathrm{~s}, 6 \mathrm{H}), 3.90(\mathrm{~s}, 3 \mathrm{H}), 3.72(\mathrm{~s}, 3 \mathrm{H}) .{ }^{13} \mathrm{C} \mathrm{NMR}(100$ $\left.\mathrm{MHz}, \mathrm{CDCl}_{3}\right) \delta 172.7,165.0,152.9,141.0,137.8,135.8,129.1,128.5,127.3,122.2,118.7,105.7$, 82.2, 71.5, 60.8, 56.3, 56.1, 52.6. HRMS (ESI-TOF) Calcd. For $\mathrm{C}_{23} \mathrm{H}_{25} \mathrm{NO}_{6}\left([\mathrm{M}+\mathrm{H}]^{+}\right): 412.1755$, found: 412.1748 . The product was analyzed by HPLC to determine the enantiomeric excess: $>99 \%$ ee $($ Chiralpak AD-H, $i$-propanol/hexane $=5 / 95$, flow rate $1.0 \mathrm{~mL} / \mathrm{min}, \lambda=220 \mathrm{~nm}) ; \mathrm{t}_{\mathrm{r}}=11.97$ and $13.13 \mathrm{~min}$.

\section{Methyl}

(S)-2-([1,1'-biphenyl]-2-yl)-4-((S)-1-phenylallyl)-4,5-dihydrooxazole-4-carboxylate

(3ea):

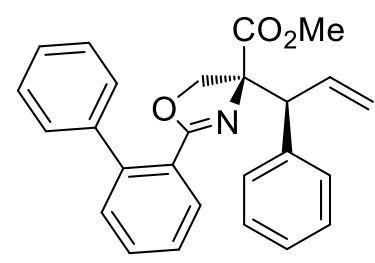

Yield (56\%); $44.5 \mathrm{mg}$; yellow oil; it was purified by column chromatography (silica gel, eluent: petroleum ether: ethyl acetate $=20: 1-10: 1) .[\alpha]^{20} \mathrm{D}=+60.3\left(c 0.30, \mathrm{CHCl}_{3}\right) ;{ }^{1} \mathrm{H}$ NMR $(400 \mathrm{MHz}$, $\left.\mathrm{CDCl}_{3}\right) \delta 7.85(\mathrm{dd}, J=7.7,1.0 \mathrm{~Hz}, 1 \mathrm{H}), 7.51(\mathrm{dt}, J=7.6,1.4 \mathrm{~Hz}, 1 \mathrm{H}), 7.43-7.35(\mathrm{~m}, 2 \mathrm{H}), 7.34-7.26$ (m, 5H), 7.26-7.19 (m, 5H), 6.34-6.25 (m, 1H), 5.22-5.12 (m, 2H), 4.37 (d, J = 9.2 Hz, 1H), 4.15 (d, $J=9.2 \mathrm{~Hz}, 1 \mathrm{H}), 3.98(\mathrm{~d}, J=8.4 \mathrm{~Hz}, 1 \mathrm{H}), 3.71(\mathrm{~s}, 3 \mathrm{H}) .{ }^{13} \mathrm{C} \mathrm{NMR}\left(100 \mathrm{MHz}, \mathrm{CDCl}_{3}\right) \delta 173.1,167.4$, $141.8,138.1,136.2,130.9,130.6,130.2,129.4,128.5,128.3,127.9,127.4,127.2,126.8,118.4,81.5$, 72.3, 55.7, 52.5. HRMS (ESI-TOF) Calcd. For $\mathrm{C}_{26} \mathrm{H}_{23} \mathrm{NO}_{3}\left([\mathrm{M}+\mathrm{H}]^{+}\right): 398.1751$, found: 398.1743 The product was analyzed by HPLC to determine the enantiomeric excess: $>99 \%$ ee (Chiralpak AD-H, $i$-propanol $/$ hexane $=5 / 95$, flow rate $1.0 \mathrm{~mL} / \mathrm{min}, \lambda=220 \mathrm{~nm}) ; \mathrm{t}_{\mathrm{r}}=6.10$ and $8.50 \mathrm{~min}$.

\section{Methyl (S)-2-(naphthalen-2-yl)-4-((S)-1-phenylallyl)-4,5-dihydrooxazole-4-carboxylate (3fa):}

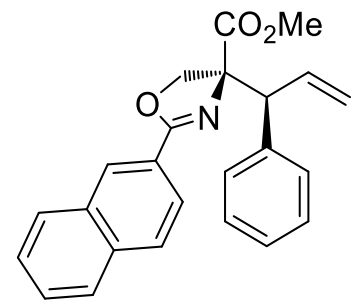

Yield (97\%); $72.0 \mathrm{mg}$; white solid; $\mathrm{mp} 100-102{ }^{\circ} \mathrm{C}$; it was purified by column chromatography (silica 
gel, eluent: petroleum ether: ethyl acetate $=25: 1-10: 1) .[\alpha]^{20} \mathrm{D}=+190.3\left(c 1.0, \mathrm{CHCl}_{3}\right) ;{ }^{1} \mathrm{H} \mathrm{NMR}$ $\left(400 \mathrm{MHz}, \mathrm{CDCl}_{3}\right) \delta 8.45(\mathrm{~s}, 1 \mathrm{H}), 8.05(\mathrm{dd}, J=8.6,1.6 \mathrm{~Hz}, 1 \mathrm{H}), 7.90-7.84(\mathrm{~m}, 3 \mathrm{H}), 7.56-7.49(\mathrm{~m}$, 2H), 7.26-7.18 (m, 5H), 6.38-6.29 (m, 1H), 5.22-5.13 (m, 2H), $4.75(\mathrm{~d}, J=9.2 \mathrm{~Hz}, 1 \mathrm{H}), 4.54(\mathrm{~d}, J=$ $9.2 \mathrm{~Hz}, 1 \mathrm{H}), 4.07(\mathrm{~d}, J=8.4 \mathrm{~Hz}, 1 \mathrm{H}), 3.73(\mathrm{~s}, 3 \mathrm{H}) .{ }^{13} \mathrm{C} \mathrm{NMR}\left(100 \mathrm{MHz}, \mathrm{CDCl}_{3}\right) \delta 173.0,165.4$, $137.8,136.0,134.8,132.5,129.2,129.1,128.9,128.4,128.0,127.69,127.66,127.3,126.5,124.9$, 124.3, 118.5, 82.2, 71.8, 56.5, 52.6. HRMS (ESI-TOF) Calcd. For $\mathrm{C}_{24} \mathrm{H}_{21} \mathrm{NO}_{3}\left([\mathrm{M}+\mathrm{H}]^{+}\right): 372.1594$, found: 372.1589 . The product was analyzed by HPLC to determine the enantiomeric excess: $>99 \%$ ee $($ Chiralpak AD-H, $i$-propanol/hexane $=5 / 95$, flow rate $1.0 \mathrm{~mL} / \mathrm{min}, \lambda=254 \mathrm{~nm}) ; \mathrm{t}_{\mathrm{r}}=8.28$ and $15.02 \mathrm{~min}$.

Methyl (S)-2-(4-fluorophenyl)-4-((S)-1-phenylallyl)-4,5-dihydrooxazole-4-carboxylate (3ga):

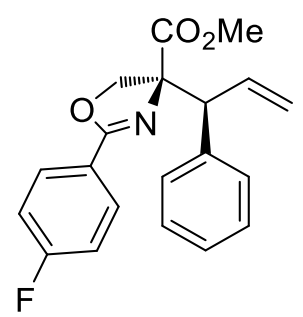

Yield (60\%); $40.7 \mathrm{mg}$; yellow oil; it was purified by column chromatography (silica gel, eluent: petroleum ether: ethyl acetate $=30: 1-20: 1) .[\alpha]^{20}{ }_{\mathrm{D}}=+76.2\left(c 0.7, \mathrm{CHCl}_{3}\right) ;{ }^{1} \mathrm{H} \mathrm{NMR}(400 \mathrm{MHz}$, $\left.\mathrm{CDCl}_{3}\right) \delta$ 7.97-7.93 (m, 2H), 7.25-7.21 (m, 5H), 7.11-7.04 (m, 2H), 6.33-6.24 (m, 1H), 5.20-5.10 (m, 2H), $4.68(\mathrm{~d}, J=9.2 \mathrm{~Hz}, 1 \mathrm{H}), 4.47(\mathrm{~d}, J=9.2 \mathrm{~Hz}, 1 \mathrm{H}), 4.02(\mathrm{~d}, J=8.4 \mathrm{~Hz}, 1 \mathrm{H}), 3.71(\mathrm{~s}, 3 \mathrm{H}) .{ }^{13} \mathrm{C}$ NMR $\left(100 \mathrm{MHz}, \mathrm{CDCl}_{3}\right) \delta 172.9,164.9(\mathrm{~d}, J=250.9 \mathrm{~Hz}), 164.4,137.8,135.9,130.85(\mathrm{~d}, J=8.9$ Hz), 129.2, 128.4, 127.4, 123.2 (d, $J=3.0 \mathrm{~Hz}), 118.5,115.4(\mathrm{~d}, J=21.8 \mathrm{~Hz}), 82.1,71.9,56.4,52.6$.

${ }^{19} \mathrm{~F}$ NMR (376 MHz, $\left.\mathrm{CDCl}_{3}\right) \delta$-107.38. HRMS (ESI-TOF) Calcd. For $\mathrm{C}_{20} \mathrm{H}_{18} \mathrm{FNO}_{3}\left([\mathrm{M}+\mathrm{H}]^{+}\right)$: 340.1343, found: 340.1337. The product was analyzed by HPLC to determine the enantiomeric excess: $95 \%$ ee (Chiralpak AD-H, $i$-propanol/hexane $=5 / 95$, flow rate $1.0 \mathrm{~mL} / \mathrm{min}, \lambda=254 \mathrm{~nm}) ; \mathrm{t}_{\mathrm{r}}=$ 6.50 and $9.67 \mathrm{~min}$.

Methyl (S)-4-((S)-1-phenylallyl)-2-(4-(trifluoromethyl) phenyl)-4,5-dihydrooxazo-le-4carboxylate (3ha): 


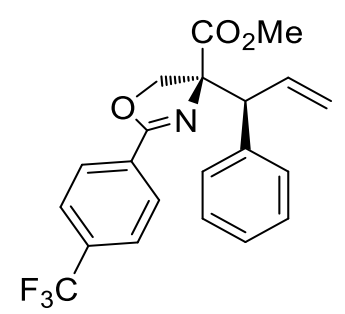

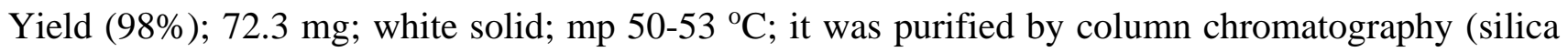
gel, eluent: petroleum ether: ethyl acetate $=15: 1) \cdot[\alpha]^{20} \mathrm{D}=+123.0\left(c\right.$ 1.1, $\left.\mathrm{CHCl}_{3}\right) ;{ }^{1} \mathrm{H}$ NMR $(400$ $\left.\mathrm{MHz}, \mathrm{CDCl}_{3}\right) \delta 8.06(\mathrm{~d}, J=8.0 \mathrm{~Hz}, 2 \mathrm{H}), 7.66(\mathrm{~d}, J=8.0 \mathrm{~Hz}, 2 \mathrm{H}), 7.23-7.20(\mathrm{~m}, 5 \mathrm{H}), 6.33-6.24(\mathrm{~m}$, $1 \mathrm{H}), 5.21-5.12(\mathrm{~m}, 2 \mathrm{H}), 4.72(\mathrm{~d}, J=9.2 \mathrm{~Hz}, 1 \mathrm{H}), 4.51(\mathrm{~d}, J=9.2 \mathrm{~Hz}, 1 \mathrm{H}), 4.03(\mathrm{~d}, J=8.4 \mathrm{~Hz}, 1 \mathrm{H})$, $3.73(\mathrm{~s}, 3 \mathrm{H}) .{ }^{13} \mathrm{C} \mathrm{NMR}\left(100 \mathrm{MHz}, \mathrm{CDCl}_{3}\right) \delta 172.7,164.1,137.7,135.8,133.4(\mathrm{q}, J=29.5 \mathrm{~Hz}), 130.4$, 129.2, 129.0, 128.5, 127.5, 125.3 (q, $J=3.6 \mathrm{~Hz}), 123.7(\mathrm{q}, J=271.0 \mathrm{~Hz}), 118.7,82.3,72.2,56.5$, 52.7. ${ }^{19} \mathrm{~F}$ NMR (376 MHz, $\left.\mathrm{CDCl}_{3}\right) \delta-63.00$. HRMS (ESI-TOF) Calcd. For $\mathrm{C}_{21} \mathrm{H}_{18} \mathrm{~F}_{3} \mathrm{NO}_{3}\left([\mathrm{M}+\mathrm{H}]^{+}\right)$: 390.1312, found: 390.1303. The product was analyzed by HPLC to determine the enantiomeric excess: $>99 \%$ ee $($ Chiralpak ID-H, $i$-propanol/hexane $=5 / 95$, flow rate $1.0 \mathrm{~mL} / \mathrm{min}, \lambda=254 \mathrm{~nm})$; $\mathrm{t}_{\mathrm{r}}=$ 5.10 and $7.10 \mathrm{~min}$.

Methyl $(R)-4-((R)-1-p h e n y l a l l y l)-2-(4-(t r i f l u o r o m e t h y l)$ phenyl)-4,5-dihydrooxazole-4-carboxy -late (3ha):<smiles>C=CC(c1ccccc1)C(COC(C)=O)(N=C(C)C)c1ccc(C(F)(F)F)cc1</smiles>

Yield (92\%); $71.5 \mathrm{mg}$; white solid; $\mathrm{mp} 50-52{ }^{\circ} \mathrm{C}$; it was purified by column chromatography (silica gel, eluent: petroleum ether: ethyl acetate $=15: 1) \cdot[\alpha]^{20} \mathrm{D}=-125.0\left(c\right.$ 1.2, $\left.\mathrm{CHCl}_{3}\right)$; The product was analyzed by HPLC to determine the enantiomeric excess: $96 \%$ ee (Chiralpak ID-H, $i$-propanol/hexane $=5 / 95$, flow rate $1.0 \mathrm{~mL} / \mathrm{min}, \lambda=254 \mathrm{~nm}) ; \mathrm{t}_{\mathrm{r}}=5.51$ and $7.37 \mathrm{~min}$.

Methyl (S)-4-((R)-1-phenylallyl)-2-(4-(trifluoromethyl) phenyl)-4,5-dihydrooxazole-4-carboxy -late (3ha): 


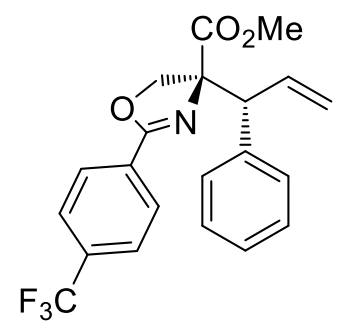

Yield (97\%); $75.4 \mathrm{mg}$; white solid; mp 50-52 ${ }^{\circ} \mathrm{C}$; it was purified by column chromatography (silica gel, eluent: petroleum ether: ethyl acetate $=15: 1) .{ }^{1} \mathrm{H} \mathrm{NMR}\left(400 \mathrm{MHz}, \mathrm{CDCl}_{3}\right) \delta 8.07(\mathrm{~d}, J=8.2 \mathrm{~Hz}$, 2H), $7.66(\mathrm{~d}, J=8.4 \mathrm{~Hz}, 2 \mathrm{H}), 7.40-7.38(\mathrm{~m}, 2 \mathrm{H}), 7.29-7.25(\mathrm{~m}, 2 \mathrm{H}), 7.21-7.19(\mathrm{~m}, 1 \mathrm{H})$, 6.06-5.96 (m, 1H), 5.26-5.22 (m, 2H), $4.79(\mathrm{~d}, J=9.2 \mathrm{~Hz}, 1 \mathrm{H}), 4.56(\mathrm{~d}, J=9.2 \mathrm{~Hz}, 1 \mathrm{H}), 3.97(\mathrm{~d}, J=9.4 \mathrm{~Hz}, 1 \mathrm{H})$, $3.64(\mathrm{~s}, 3 \mathrm{H}) .{ }^{13} \mathrm{C} \mathrm{NMR}\left(100 \mathrm{MHz}, \mathrm{CDCl}_{3}\right) \delta 172.7,164.1,137.7,135.8,133.3$ (q, $\left.J=32.4 \mathrm{~Hz}\right), 130.4$, 129.2, 129.0, 128.5, 127.5, 125.4 (q, $J=3.6 \mathrm{~Hz}), 123.7$ (q, $J=271.0 \mathrm{~Hz}), 118.7,82.3,72.2,56.6$, 52.7. ${ }^{19} \mathrm{~F}$ NMR $\left(376 \mathrm{MHz}, \mathrm{CDCl}_{3}\right) \delta-63.00$. The product was analyzed by HPLC to determine the enantiomeric excess: $>99 \%$ ee (Chiralpak AD-H, $i$-propanol $/$ hexane $=5 / 95$, flow rate $1.0 \mathrm{~mL} / \mathrm{min}, \lambda$ $=254 \mathrm{~nm}) ; \mathrm{t}_{\mathrm{r}}=5.43$ and $9.21 \mathrm{~min}$.

\section{Methyl (R)-4-((S)-1-phenylallyl)-2-(4-(trifluoromethyl) phenyl)-4,5-dihydrooxazole-4-carboxy} -late (3ha)<smiles>C=C[C@H](c1ccccc1)C(COC)(N=C(C)C)c1ccc(C(F)(F)F)cc1</smiles>

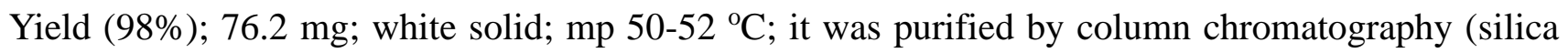
gel, eluent: petroleum ether: ethyl acetate $=15: 1$ ). The product was analyzed by HPLC to determine the enantiomeric excess: $94 \%$ ee (Chiralpak AD-H, $i$-propanol $/$ hexane $=5 / 95$, flow rate $1.0 \mathrm{~mL} / \mathrm{min}$, $\lambda=254 \mathrm{~nm}) ; \mathrm{t}_{\mathrm{r}}=5.53$ and $9.90 \mathrm{~min}$.

\section{Methyl (R)-2-phenyl-4-((S)-1-phenylallyl)-4,5-dihydrothiazole-4-carboxylate (3ia):}<smiles>C=CC(c1ccccc1)[C@](CS)(CSc1ccccc1)C(C)=O</smiles> 
Yield (96\%); $64.7 \mathrm{mg}$; colorless oil; it was purified by column chromatography (silica gel, eluent: petroleum ether: ethyl acetate $=25: 1-10: 1) .[\alpha]^{20} \mathrm{D}=+120.7\left(c\right.$ 0.9, $\left.\mathrm{CHCl}_{3}\right) ;{ }^{1} \mathrm{H}$ NMR $(400 \mathrm{MHz}$, $\left.\mathrm{CDCl}_{3}\right) \delta$ 7.80-7.77 (m, 2H), 7.42-7.36 (m, 1H), 7.36-7.30 (m, 2H), 7.18-7.16 (m, 5H), 6.42-6.33 (m, 1H), $5.11(\mathrm{~s}, 1 \mathrm{H}), 5.09-5.06(\mathrm{~m}, 1 \mathrm{H}), 4.00(\mathrm{~d}, J=8.8 \mathrm{~Hz}, 1 \mathrm{H}), 3.72(\mathrm{~d}, J=11.6 \mathrm{~Hz}, 1 \mathrm{H}), 3.63(\mathrm{~s}, 3 \mathrm{H})$, $3.39(\mathrm{~d}, J=11.6 \mathrm{~Hz}, 1 \mathrm{H}) .{ }^{13} \mathrm{C} \mathrm{NMR}\left(100 \mathrm{MHz}, \mathrm{CDCl}_{3}\right) \delta 173.4,170.0,138.5,137.4,133.0,131.5$, 129.6, 128.55, 128.49, 128.4, 127.3, 117.8, 92.5, 57.9, 52.7, 38.9. HRMS (ESI-TOF) Calcd. For $\mathrm{C}_{20} \mathrm{H}_{19} \mathrm{NO}_{2} \mathrm{~S}\left([\mathrm{M}+\mathrm{H}]^{+}\right)$: 338.1209 , found: 338.1201 . The product was analyzed by HPLC to determine the enantiomeric excess: $98 \%$ ee (Chiralpak ID-H, $i$-propanol/hexane $=2 / 98$, flow rate 1.0 $\mathrm{mL} / \mathrm{min}, \lambda=254 \mathrm{~nm}) ; \mathrm{t}_{\mathrm{r}}=6.62$ and $7.53 \mathrm{~min}$.

\section{Methyl (S)-2-phenyl-4-((R)-1-phenylallyl)-4,5-dihydrothiazole-4-carboxylate (3ia):}

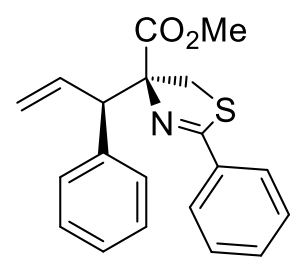

Yield (84\%); $56.6 \mathrm{mg}$; colorless oil; it was purified by column chromatography (silica gel, eluent: petroleum ether: ethyl acetate $=25: 1-10: 1) \cdot[\alpha]^{20}{ }_{\mathrm{D}}=-122.7\left(c\right.$ 1.3, $\left.\mathrm{CHCl}_{3}\right)$; The product was analyzed by HPLC to determine the enantiomeric excess: $>99 \%$ ee (Chiralpak ID-H, $i$-propanol/hexane $=2 / 98$, flow rate $1.0 \mathrm{~mL} / \mathrm{min}, \lambda=254 \mathrm{~nm}$ ); $\mathrm{t}_{\mathrm{r}}=6.62$ and $7.53 \mathrm{~min}$.

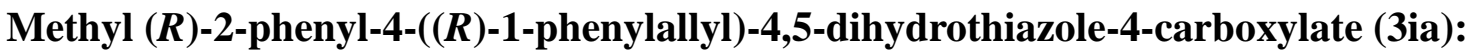

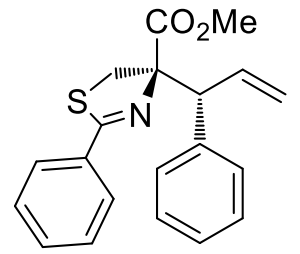

Yield (84\%); $56.6 \mathrm{mg}$; colorless oil; it was purified by column chromatography (silica gel, eluent: petroleum ether: ethyl acetate $=25: 1-10: 1) .{ }^{1} \mathrm{H}$ NMR $\left(400 \mathrm{MHz}, \mathrm{CDCl}_{3}\right) \delta$ 7.90-7.87 (m, 2H), 7.47-7.41 (m, 5H), 7.30-7.18 (m, 3H), 6.15-6.06 (m, 1H), 5.24-5.18 (m, 2H), $4.01(\mathrm{~d}, J=9.6 \mathrm{~Hz}, 1 \mathrm{H})$, $3.92(\mathrm{~d}, J=11.6 \mathrm{~Hz}, 1 \mathrm{H}), 3.62(\mathrm{~d}, J=11.6 \mathrm{~Hz}, 1 \mathrm{H}), 3.53(\mathrm{~s}, 3 \mathrm{H}) .{ }^{13} \mathrm{C} \mathrm{NMR}\left(100 \mathrm{MHz}, \mathrm{CDCl}_{3}\right) \delta$ $173.0,169.6,139.4,135.9,132.9,131.5,129.2,128.5,128.4,128.1,127.0,118.6,91.9,58.1,52.5$, 
39.0. The product was analyzed by HPLC to determine the enantiomeric excess: $95 \%$ ee (Chiralpak ID-H, $i$-propanol $/$ hexane $=2 / 98$, flow rate $1.0 \mathrm{~mL} / \mathrm{min}, \lambda=254 \mathrm{~nm}$ ); $\mathrm{t}_{\mathrm{r}}=6.66$ and $7.89 \mathrm{~min}$.

\section{Methyl (S)-2-phenyl-4-((S)-1-phenylallyl)-4,5-dihydrothiazole-4-carboxylate (3ia):}

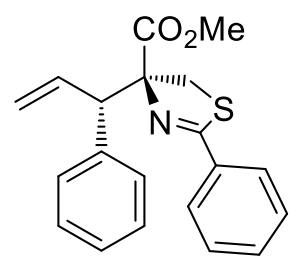

Yield (96\%); $64.7 \mathrm{mg}$; colorless oil; it was purified by column chromatography (silica gel, eluent: petroleum ether: ethyl acetate $=25: 1-10: 1)$. The product was analyzed by HPLC to determine the enantiomeric excess: $>99 \%$ ee (Chiralpak ID-H, $i$-propanol $/$ hexane $=2 / 98$, flow rate $1.0 \mathrm{~mL} / \mathrm{min}, \lambda=$ $254 \mathrm{~nm}) ; \mathrm{t}_{\mathrm{r}}=6.26$ and $6.92 \mathrm{~min}$.

\section{Methyl}

(S)-4-((S)-1-(p-tolyl)

allyl)-2-(4-(trifluoromethyl)

phenyl)-4,5-dihydrooxazole-4-carboxylate (3hb):

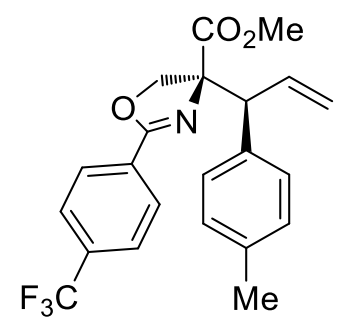

Yield (90\%); $72.5 \mathrm{mg}$; yellow oil; it was purified by column chromatography (silica gel, eluent: petroleum ether: ethyl acetate $=25: 1) .[\alpha]^{20} \mathrm{D}=+118.0\left(c 0.7, \mathrm{CHCl}_{3}\right) ;{ }^{1} \mathrm{H} \mathrm{NMR}\left(400 \mathrm{MHz}, \mathrm{CDCl}_{3}\right) \delta$ $8.07(\mathrm{~d}, J=8.0 \mathrm{~Hz}, 2 \mathrm{H}), 7.66(\mathrm{~d}, J=8.4 \mathrm{~Hz}, 2 \mathrm{H}), 7.10-7.04(\mathrm{~m}, 4 \mathrm{H}), 6.31-6.22(\mathrm{~m}, 1 \mathrm{H}), 5.19-5.11$ $(\mathrm{m}, 2 \mathrm{H}), 4.72(\mathrm{~d}, J=9.2 \mathrm{~Hz}, 1 \mathrm{H}), 4.51(\mathrm{~d}, J=9.2 \mathrm{~Hz}, 1 \mathrm{H}), 4.00(\mathrm{~d}, J=8.4 \mathrm{~Hz}, 1 \mathrm{H}), 3.73(\mathrm{~s}, 3 \mathrm{H})$, $2.27(\mathrm{~s}, 3 \mathrm{H}) .{ }^{13} \mathrm{C} \mathrm{NMR}\left(100 \mathrm{MHz}, \mathrm{CDCl}_{3}\right) \delta 172.7,164.0,137.0,135.9,134.6,133.3$ (q, $\left.J=32.4 \mathrm{~Hz}\right)$, 130.4, 129.2, 129.0, 128.9, 125.2 (q, $J=3.6 \mathrm{~Hz}), 123.7$ (q, $J=273.6 \mathrm{~Hz}$ ), 118.4, 82.3, 72.2, 56.0, 52.6, 21.0. ${ }^{19} \mathrm{~F}$ NMR (376 MHz, $\left.\mathrm{CDCl}_{3}\right) \delta$-62.99. HRMS (ESI-TOF) Calcd. For $\mathrm{C}_{22} \mathrm{H}_{20} \mathrm{~F}_{3} \mathrm{NO}_{3}$ $\left([\mathrm{M}+\mathrm{H}]^{+}\right): 404.1468$, found: 404.1459 . The product was analyzed by HPLC to determine the enantiomeric excess: $>99 \%$ ee (Chiralpak ID-H, $i$-propanol $/$ hexane $=2 / 98$, flow rate $1.0 \mathrm{~mL} / \mathrm{min}, \lambda=$ $254 \mathrm{~nm}) ; \mathrm{t}_{\mathrm{r}}=5.61$ and $8.48 \mathrm{~min}$. 


\section{phenyl)-4,5-dihydrooxazole-4-carboxylate (3hc):}<smiles>C=CC(c1ccc(Cl)cc1)C(C)(C)C(C)=NC(=O)c1ccc(C(F)(F)F)cc1</smiles>

Yield (97\%); $82.1 \mathrm{mg}$; white solid; $\mathrm{mp} 90-92{ }^{\circ} \mathrm{C}$; it was purified by column chromatography (silica gel, eluent: petroleum ether: ethyl acetate $=25: 1)$. $[\alpha]^{20} \mathrm{D}=+130.2\left(c 1.0, \mathrm{CHCl}_{3}\right) ;{ }^{1} \mathrm{H}$ NMR $(400$ $\left.\mathrm{MHz} \mathrm{CDCl}_{3}\right) \delta 8.06(\mathrm{~d}, J=8.4 \mathrm{~Hz}, 2 \mathrm{H}), 7.67(\mathrm{~d}, J=8.4 \mathrm{~Hz}, 2 \mathrm{H}), 7.22(\mathrm{~d}, J=8.8 \mathrm{~Hz}, 2 \mathrm{H}), 7.14(\mathrm{~d}, J$ $=8.4 \mathrm{~Hz}, 2 \mathrm{H}), 6.29-6.20(\mathrm{~m}, 1 \mathrm{H}), 5.23-5.11(\mathrm{~m}, 2 \mathrm{H}), 4.70(\mathrm{~d}, J=9.2 \mathrm{~Hz}, 1 \mathrm{H}), 4.44(\mathrm{~d}, J=9.2 \mathrm{~Hz}$, $1 \mathrm{H}), 3.99(\mathrm{~d}, J=8.4 \mathrm{~Hz}, 1 \mathrm{H}), 3.73(\mathrm{~s}, 3 \mathrm{H}) .{ }^{13} \mathrm{C} \mathrm{NMR}\left(100 \mathrm{MHz}, \mathrm{CDCl}_{3}\right) \delta 172.6,164.3,136.3,135.4$, $133.5(\mathrm{q}, J=32.8 \mathrm{~Hz}), 133.4,130.5,130.2,129.0,128.7,125.4(\mathrm{q}, J=3.5 \mathrm{~Hz}), 123.7$ (q, $J=273.7$ $\mathrm{Hz}), 119.0,82.1,72.3,55.9,52.8 .{ }^{19} \mathrm{~F}$ NMR (376 MHz, $\left.\mathrm{CDCl}_{3}\right) \delta$-62.99. HRMS (ESI-TOF) Calcd. For $\mathrm{C}_{21} \mathrm{H}_{17} \mathrm{ClF}_{3} \mathrm{NO}_{3}\left([\mathrm{M}+\mathrm{H}]^{+}\right)$: 424.0922, found: 424.0915 . The product was analyzed by HPLC to determine the enantiomeric excess: $>99 \%$ ee (Chiralpak AD-H, $i$-propanol/hexane $=5 / 95$, flow rate $1.0 \mathrm{~mL} / \mathrm{min}, \lambda=254 \mathrm{~nm}) ; \mathrm{t}_{\mathrm{r}}=7.19$ and $7.94 \mathrm{~min}$.

Methyl

(S)-4-((S)-1-(4-methoxyphenyl)

allyl)-2-(4-(trifluoromethyl)

phenyl)-4,5-dihydrooxazole-4-carboxylate (3hd):

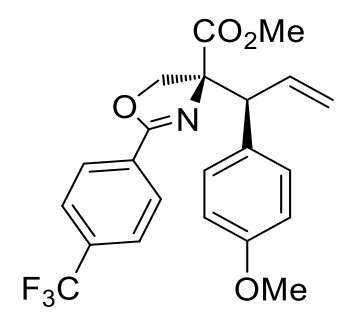

Yield (70\%); $58.7 \mathrm{mg}$; yellow oil; it was purified by column chromatography (silica gel, eluent: petroleum ether: ethyl acetate $=25: 1)$. $[\alpha]^{20}{ }_{\mathrm{D}}=+109.6\left(\right.$ c 2.2, $\left.\mathrm{CHCl}_{3}\right) ;{ }^{1} \mathrm{H} \mathrm{NMR}\left(400 \mathrm{MHz}, \mathrm{CDCl}_{3}\right) \delta$ $8.06(\mathrm{~d}, J=8.4 \mathrm{~Hz}, 2 \mathrm{H}), 7.66(\mathrm{~d}, J=8.4 \mathrm{~Hz}, 2 \mathrm{H}), 7.12(\mathrm{~d}, J=8.8 \mathrm{~Hz}, 2 \mathrm{H}), 6.77(\mathrm{~d}, J=8.8 \mathrm{~Hz}, 2 \mathrm{H})$, 6.30-6.21 (m, 1H), 5.19-5.10 (m, 2H), $4.70(\mathrm{~d}, J=9.2 \mathrm{~Hz}, 1 \mathrm{H}), 4.48(\mathrm{~d}, J=9.2 \mathrm{~Hz}, 1 \mathrm{H}), 3.97(\mathrm{~d}, J=$ $8.4 \mathrm{~Hz}, 1 \mathrm{H}), 3.74(\mathrm{~s}, 3 \mathrm{H}), 3.73(\mathrm{~s}, 3 \mathrm{H}) .{ }^{13} \mathrm{C} \mathrm{NMR}\left(100 \mathrm{MHz}, \mathrm{CDCl}_{3}\right) \delta 172.8,164.0,158.8,136.0$, $133.3(\mathrm{q}, J=32.4 \mathrm{~Hz}), 130.4,130.2,129.6,128.9,125.3$ (q, $J=3.7 \mathrm{~Hz}), 123.7$ (q, $J=273.6 \mathrm{~Hz})$, 
118.3, 113.9, 82.4, 72.2, 55.7, 55.1, 52.6. ${ }^{19} \mathrm{~F}$ NMR (376 MHz, $\left.\mathrm{CDCl}_{3}\right) \delta-62.99$. HRMS (ESI-TOF) Calcd. For $\mathrm{C}_{22} \mathrm{H}_{20} \mathrm{~F}_{3} \mathrm{NO}_{4}\left([\mathrm{M}+\mathrm{H}]^{+}\right)$: 420.1417, found: 420.1412. The product was analyzed by HPLC to determine the enantiomeric excess: $>99 \%$ ee (Chiralpak AD-H, $i$-propanol/hexane $=5 / 95$, flow rate $1.0 \mathrm{~mL} / \mathrm{min}, \lambda=254 \mathrm{~nm}) ; \mathrm{t}_{\mathrm{r}}=9.22$ and $11.59 \mathrm{~min}$.

\section{Methyl}

(S)-4-((S)-1-(4-bromophenyl)

allyl)-2-(4-(trifluoromethyl)

phenyl)-4,5-dihydrooxazole-4-carboxylate (3he):<smiles>C=CC(c1ccc(Br)cc1)C(C)(C)c1nc(-c2ccc(C(F)(F)F)cc2)co1</smiles>

Yield (75\%); $70.1 \mathrm{mg}$; white solid; $\mathrm{mp} 102-105^{\circ} \mathrm{C}$; it was purified by column chromatography (silica gel, eluent: petroleum ether: ethyl acetate $=25: 1) .[\alpha]^{20} \mathrm{D}=+124.1\left(c 1.1, \mathrm{CHCl}_{3}\right) ;{ }^{1} \mathrm{H}$ NMR $(400$ $\left.\mathrm{MHz} \mathrm{CDCl}_{3}\right) \delta 8.06(\mathrm{~d}, J=8.0 \mathrm{~Hz}, 2 \mathrm{H}), 7.67(\mathrm{~d}, J=8.2 \mathrm{~Hz}, 2 \mathrm{H}), 7.38-7.36(\mathrm{~m}, 2 \mathrm{H}), 7.10-7.08(\mathrm{~m}$, 2H), 6.28-6.19 (m, 1H), 5.22-5.11 (m, 2H), $4.70(\mathrm{~d}, J=9.2 \mathrm{~Hz}, 1 \mathrm{H}), 4.43(\mathrm{~d}, J=9.2 \mathrm{~Hz}, 1 \mathrm{H}), 3.98(\mathrm{~d}$, $J=8.4 \mathrm{~Hz}, 1 \mathrm{H}), 3.73(\mathrm{~s}, 3 \mathrm{H}) .{ }^{13} \mathrm{C} \mathrm{NMR}\left(100 \mathrm{MHz}, \mathrm{CDCl}_{3}\right) \delta 172.5,164.3,136.8,135.4,133.5(\mathrm{q}, J$ = 32.7 Hz), 131.6, 130.9, 130.2, 129.0, 125.4 (q, $J=3.7 \mathrm{~Hz}), 123.7$ (q, $J=273.7 \mathrm{~Hz}), 121.6,119.0$, 82.0, 72.3, 55.9, 52.7. ${ }^{19} \mathrm{~F}$ NMR $\left(376 \mathrm{MHz}, \mathrm{CDCl}_{3}\right) \delta$-63.02. HRMS (ESI-TOF) Calcd. For $\mathrm{C}_{21} \mathrm{H}_{17} \mathrm{BrF}_{3} \mathrm{NO}_{3}\left([\mathrm{M}+\mathrm{H}]^{+}\right): 468.0417$, found: 468.0407 . The product was analyzed by HPLC to determine the enantiomeric excess: $>99 \%$ ee (Chiralpak ID-H, $i$-propanol/hexane $=2 / 98$, flow rate $1.0 \mathrm{~mL} / \mathrm{min}, \lambda=254 \mathrm{~nm}$ ); $\mathrm{t}_{\mathrm{r}}=5.48$ and $9.85 \mathrm{~min}$.

Methyl

(S)-4-((S)-1-(3,4-dichlorophenyl)

allyl)-2-(4-(trifluoromethyl)phenyl)-4,5-

dihydrooxazole-4-carboxylate (3hf):<smiles>C=CC(c1ccc(Cl)c(Cl)c1)[C@]1(C)COC(c2ccc(C(F)(F)F)cc2)=N[C@@H]1C(C)=O</smiles>

Yield (69\%); $63.1 \mathrm{mg}$; yellow oil; it was purified by column chromatography (silica gel, eluent: 
petroleum ether: ethyl acetate $=25: 1) \cdot[\alpha]^{20} \mathrm{D}=+118.4\left(c 1.0, \mathrm{CHCl}_{3}\right) ;{ }^{1} \mathrm{H} \mathrm{NMR}\left(400 \mathrm{MHz}, \mathrm{CDCl}_{3}\right) \delta$ $8.06(\mathrm{~d}, J=8.0 \mathrm{~Hz}, 2 \mathrm{H}), 7.67(\mathrm{~d}, J=8.4 \mathrm{~Hz}, 2 \mathrm{H}), 7.33-7.30(\mathrm{~m}, 2 \mathrm{H}), 7.06(\mathrm{dd}, J=8.3,2.1 \mathrm{~Hz}, 1 \mathrm{H})$, 6.28-6.19 (m, 1H), 5.24-5.13 (m, 2H), 4.68 (d, $J=9.6 \mathrm{~Hz}, 1 \mathrm{H}), 4.41$ (d, $J=9.6 \mathrm{~Hz}, 1 \mathrm{H}), 3.94(\mathrm{~d}, J=$ $8.4 \mathrm{~Hz}, 1 \mathrm{H}), 3.75(\mathrm{~s}, 3 \mathrm{H}) .{ }^{13} \mathrm{C} \mathrm{NMR}\left(100 \mathrm{MHz}, \mathrm{CDCl}_{3}\right) \delta 172.3,164.5,138.1,134.9,133.6(\mathrm{q}, J=$ $32.6 \mathrm{~Hz}), 132.5,131.6,131.3,130.3,130.0,129.0,128.4,125.4$ (q, $J=3.7 \mathrm{~Hz}), 123.6$ (q, $J=273.5$ $\mathrm{Hz}), 119.4,81.9,72.5,55.7,52.8 .{ }^{19} \mathrm{~F}$ NMR $\left(376 \mathrm{MHz}, \mathrm{CDCl}_{3}\right) \delta-63.03$. HRMS (ESI-TOF) Calcd. For $\mathrm{C}_{21} \mathrm{H}_{16} \mathrm{Cl}_{2} \mathrm{~F}_{3} \mathrm{NO}_{3}\left([\mathrm{M}+\mathrm{H}]^{+}\right): 458.0532$, found: 458.0522 . The product was analyzed by HPLC to determine the enantiomeric excess: $>99 \%$ ee (Chiralpak ID-H, $i$-propanol/hexane $=2 / 98$, flow rate $1.0 \mathrm{~mL} / \mathrm{min}, \lambda=254 \mathrm{~nm}) ; \mathrm{t}_{\mathrm{r}}=5.47$ and $7.99 \mathrm{~min}$.

Methyl

(S)-4-((S)-1-(3-chlorophenyl)

allyl)-2-(4-(trifluoromethyl)

phenyl)-4,5-dihydrooxazole-4-carboxylate (3hg):

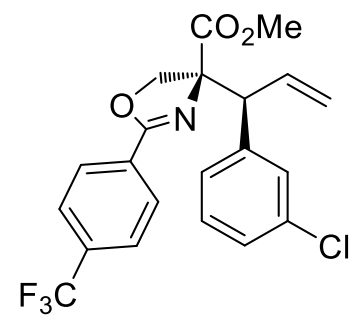

Yield (75\%); $63.5 \mathrm{mg}$; yellow oil; it was purified by column chromatography (silica gel, eluent: petroleum ether: ethyl acetate $=25: 1) \cdot[\alpha]^{20} \mathrm{D}=+122.2\left(c 0.8, \mathrm{CHCl}_{3}\right) ;{ }^{1} \mathrm{H} \mathrm{NMR}\left(400 \mathrm{MHz}, \mathrm{CDCl}_{3}\right) \delta$ $8.06(\mathrm{~d}, J=8.4 \mathrm{~Hz}, 2 \mathrm{H}), 7.66(\mathrm{~d}, J=8.4 \mathrm{~Hz}, 2 \mathrm{H}), 7.23-7.22(\mathrm{~m}, 1 \mathrm{H}), 7.20-7.14(\mathrm{~m}, 2 \mathrm{H}), 7.11-7.08$ (m, 1H), 6.27-6.22 (m, 1H), 5.23-5.13 (m, 2H), 4.69 (d, J = 9.2 Hz, 1H), $4.46(\mathrm{~d}, J=9.2 \mathrm{~Hz}, 1 \mathrm{H})$, $3.98(\mathrm{~d}, J=8.8 \mathrm{~Hz}, 1 \mathrm{H}), 3.74(\mathrm{~s}, 3 \mathrm{H}) .{ }^{13} \mathrm{C} \mathrm{NMR}\left(100 \mathrm{MHz}, \mathrm{CDCl}_{3}\right) \delta 172.4,164.4,139.8,135.2$, $134.2,133.3(\mathrm{q}, J=32.7 \mathrm{~Hz}), 130.2,129.6,129.5,129.0,127.7(\mathrm{~d}, J=9.3 \mathrm{~Hz}), 127.2,125.3(\mathrm{q}, J=$ $3.7 \mathrm{~Hz}), 123.7$ (q, $J=273.4 \mathrm{~Hz}), 119.2,82.0,72.3,56.2,52.7,{ }^{19} \mathrm{~F} \mathrm{NMR}\left(376 \mathrm{MHz}, \mathrm{CDCl}_{3}\right) \delta-63.02$. HRMS (ESI-TOF) Calcd. For $\mathrm{C}_{21} \mathrm{H}_{17} \mathrm{ClF}_{3} \mathrm{NO}_{3}\left([\mathrm{M}+\mathrm{H}]^{+}\right)$: 424.0922, found: 424.0916. The product was analyzed by HPLC to determine the enantiomeric excess: >99\% ee (Chiralpak ID-H, $i$-propanol $/$ hexane $=2 / 98$, flow rate $1.0 \mathrm{~mL} / \mathrm{min}, \lambda=254 \mathrm{~nm}$ ); $\mathrm{t}_{\mathrm{r}}=5.67$ and $7.72 \mathrm{~min}$.

\footnotetext{
Methyl

(S)-4-((S)-1-(m-tolyl)

allyl)-2-(4-(trifluoromethyl)

phenyl)-4,5-dihydrooxa

-zole-4-carboxylate (3hh):
} 


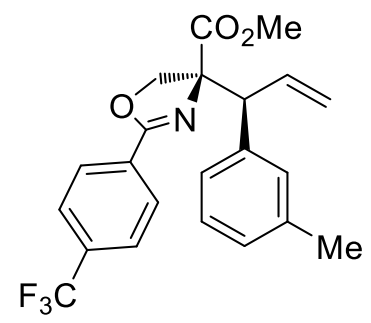

Yield (65\%); $52.4 \mathrm{mg}$; yellow oil; it was purified by column chromatography (silica gel, eluent: petroleum ether: ethyl acetate $=25: 1) .[\alpha]^{20}{ }_{\mathrm{D}}=+64.5\left(c 1.0, \mathrm{CHCl}_{3}\right) ;{ }^{1} \mathrm{H} \mathrm{NMR}\left(400 \mathrm{MHz}, \mathrm{CDCl}_{3}\right) \delta$ $8.06(\mathrm{~d}, J=8.0 \mathrm{~Hz}, 2 \mathrm{H}), 7.66(\mathrm{~d}, J=8.4 \mathrm{~Hz}, 2 \mathrm{H}), 7.16-7.08(\mathrm{~m}, 1 \mathrm{H}), 7.02-7.00(\mathrm{~m}, 3 \mathrm{H}), 6.30-6.24$ $(\mathrm{m}, 1 \mathrm{H}), 5.19-5.13(\mathrm{~m}, 2 \mathrm{H}), 4.69(\mathrm{~d}, J=9.2 \mathrm{~Hz}, 1 \mathrm{H}), 4.50(\mathrm{~d}, J=9.2 \mathrm{~Hz}, 1 \mathrm{H}), 3.98(\mathrm{~d}, J=8.8 \mathrm{~Hz}$, 1H), $3.74(\mathrm{~s}, 3 \mathrm{H}), 2.21(\mathrm{~s}, 3 \mathrm{H}) .{ }^{13} \mathrm{C} \mathrm{NMR}\left(100 \mathrm{MHz}, \mathrm{CDCl}_{3}\right) \delta 172.7,164.0,137.9,137.5,135.9$, $133.3(\mathrm{q}, J=32.6 \mathrm{~Hz}), 130.0,128.9,128.3,128.1,127.4,126.1,125.2(\mathrm{q}, J=3.7 \mathrm{~Hz}), 123.7(\mathrm{q}, J=$ 273.6 Hz), 118.5, 82.3, 72.2, 56.5, 52.6, 21.3. $\left.{ }^{19} \mathrm{~F} \mathrm{NMR} \mathrm{(376} \mathrm{MHz,} \mathrm{CDCl}_{3}\right) \delta$-62.99. HRMS (ESI-TOF) Calcd. For $\mathrm{C}_{22} \mathrm{H}_{20} \mathrm{~F}_{3} \mathrm{NO}_{3}\left([\mathrm{M}+\mathrm{H}]^{+}\right)$: 404.1468, found: 404.1462. The product was analyzed by HPLC to determine the enantiomeric excess: >99\% ee (Chiralpak ID-H, $i$-propanol $/$ hexane $=2 / 98$, flow rate $1.0 \mathrm{~mL} / \mathrm{min}, \lambda=254 \mathrm{~nm}) ; \mathrm{t}_{\mathrm{r}}=5.77$ and $8.27 \mathrm{~min}$.

\section{Methyl}

phenyl)-4,5-dihydrooxazole-4-carboxylate (3hi):

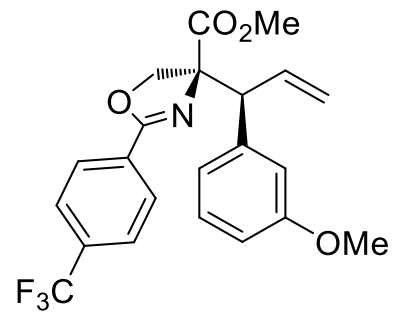

(S)-4-((S)-1-(3-methoxyphenyl)

allyl)-2-(4-(trifluoromethyl)

Yield (87\%); $75.5 \mathrm{mg}$; yellow oil; it was purified by column chromatography (silica gel, eluent: petroleum ether: ethyl acetate $=20: 1) .[\alpha]^{20} \mathrm{D}=+113.2\left(c 2.0, \mathrm{CHCl}_{3}\right) ;{ }^{1} \mathrm{H} \mathrm{NMR}\left(400 \mathrm{MHz}, \mathrm{CDCl}_{3}\right) \delta$ $8.07(\mathrm{~d}, J=8.0 \mathrm{~Hz}, 2 \mathrm{H}), 7.66(\mathrm{~d}, J=8.2 \mathrm{~Hz}, 2 \mathrm{H}), 7.18-7.14(\mathrm{~d}, J=0.8 \mathrm{~Hz}, 1 \mathrm{H}), 6.80-6.74(\mathrm{~m}, 3 \mathrm{H})$, 6.31-6.22 (m, 1H), 5.20-5.13 (m, 2H), $4.71(\mathrm{~d}, J=9.2 \mathrm{~Hz}, 1 \mathrm{H}), 4.51(\mathrm{~d}, J=9.2 \mathrm{~Hz}, 1 \mathrm{H}), 4.00(\mathrm{~d}, J=$ $8.4 \mathrm{~Hz}, 1 \mathrm{H}), 3.74(\mathrm{~s}, 3 \mathrm{H}), 3.65$ (s, 3H). ${ }^{13} \mathrm{C} \mathrm{NMR}\left(100 \mathrm{MHz}, \mathrm{CDCl}_{3}\right) \delta 172.7,164.1,159.5,139.2$, $135.7,133.3(\mathrm{q}, J=32.8 \mathrm{~Hz}), 130.4,129.4,128.9,125.3$ (q, $J=3.8 \mathrm{~Hz}), 123.7$ (q, $J=273.4 \mathrm{~Hz})$, $121.5,118.6,114.9,112.9,82.272 .2,56.4,54.9,52.6 .{ }^{19} \mathrm{~F} \mathrm{NMR}\left(376 \mathrm{MHz}, \mathrm{CDCl}_{3}\right) \delta-63.01 . \mathrm{HRMS}$ (ESI-TOF) Calcd. For $\mathrm{C}_{22} \mathrm{H}_{20} \mathrm{~F}_{3} \mathrm{NO}_{4}\left([\mathrm{M}+\mathrm{H}]^{+}\right)$: 420.1417, found: 420.1410. The product was 
analyzed by HPLC to determine the enantiomeric excess: $>99 \%$ ee (Chiralpak ID-H, $i$-propanol $/$ hexane $=2 / 98$, flow rate $1.0 \mathrm{~mL} / \mathrm{min}, \lambda=254 \mathrm{~nm}) ; \mathrm{t}_{\mathrm{r}}=7.49$ and $9.70 \mathrm{~min}$.

Methyl

(S)-4-((S)-1-(2-fluorophenyl)

allyl)-2-(4-(trifluoromethyl)

phenyl)-4,5-dihydrooxazole-4-carboxylate $(3 \mathrm{hj})$ :

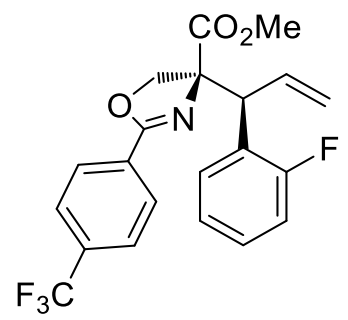

Yield (80\%); $65.1 \mathrm{mg}$; yellow oil; it was purified by column chromatography (silica gel, eluent: petroleum ether: ethyl acetate $=30: 1) .[\alpha]^{20}{ }_{\mathrm{D}}=+98.7\left(c 1.03, \mathrm{CHCl}_{3}\right) ;{ }^{1} \mathrm{H} \mathrm{NMR}\left(400 \mathrm{MHz}, \mathrm{CDCl}_{3}\right) \delta$ $8.05(\mathrm{~d}, J=8.0 \mathrm{~Hz}, 2 \mathrm{H}), 7.66(\mathrm{~d}, J=8.4 \mathrm{~Hz}, 2 \mathrm{H}), 7.21-7.19(\mathrm{~m}, 2 \mathrm{H}), 7.07-7.02(\mathrm{~m}, 1 \mathrm{H}), 6.98-6.94$ (m, 1H), 6.32-6.23 (m, 1H), 5.22-5.15 (m, 2H), $4.71(\mathrm{~d}, J=9.2 \mathrm{~Hz}, 1 \mathrm{H}), 4.50-4.46(\mathrm{~m}, 2 \mathrm{H}), 3.73$ (s, $3 \mathrm{H}) .{ }^{13} \mathrm{C} \mathrm{NMR}\left(100 \mathrm{MHz}, \mathrm{CDCl}_{3}\right) \delta 172.4,164.3,160.6(\mathrm{~d}, J=246.0 \mathrm{~Hz}), 134.8,133.4(\mathrm{q}, J=32.7$ Hz), $130.7(\mathrm{~d}, J=3.9 \mathrm{~Hz}), 130.3,129.0,128.9,125.3(\mathrm{q}, J=3.8 \mathrm{~Hz}), 124.8$ (d, $J=14.4 \mathrm{~Hz}), 124.0(\mathrm{~d}$, $J=3.5 \mathrm{~Hz}), 123.7(\mathrm{q}, J=273.6 \mathrm{~Hz}), 119.1,115.6(\mathrm{~d}, J=23.5 \mathrm{~Hz}), 82.1,72.6(\mathrm{~d}, J=4.4 \mathrm{~Hz}), 52.7$, $48.6(\mathrm{~d}, J=1.8 \mathrm{~Hz}) .{ }^{19} \mathrm{~F}$ NMR $\left(376 \mathrm{MHz}, \mathrm{CDCl}_{3}\right) \delta-63.01,-116.39$. HRMS (ESI-TOF) Calcd. For $\mathrm{C}_{21} \mathrm{H}_{17} \mathrm{~F}_{4} \mathrm{NO}_{3}\left([\mathrm{M}+\mathrm{H}]^{+}\right)$: 408.1217, found: 408.1209. The product was analyzed by HPLC to determine the enantiomeric excess: $>99 \%$ ee (Chiralpak ID-H, $i$-propanol/hexane $=2 / 98$, flow rate $1.0 \mathrm{~mL} / \mathrm{min}, \lambda=254 \mathrm{~nm}) ; \mathrm{t}_{\mathrm{r}}=5.27$ and $9.32 \mathrm{~min}$.

Methyl

(S)-4-((S)-1-(naphthalen-2-yl)

allyl)-2-(4-(trifluoromethyl)

\section{phenyl)-4,5-dihydrooxazole-4-carboxylate (3hk):}<smiles>C=CC(c1ccc2ccccc2c1)C(C)(C)C(C)=NC(=O)c1ccc(C(F)(F)F)cc1</smiles>

Yield (78\%); $68.5 \mathrm{mg}$; white solid; mp 98-100 ${ }^{\circ} \mathrm{C}$; it was purified by column chromatography (silica gel, eluent: petroleum ether: ethyl acetate $=25: 1) .[\alpha]^{20} \mathrm{D}=+102.3\left(c 0.7, \mathrm{CHCl}_{3}\right) ;{ }^{1} \mathrm{H} \mathrm{NMR}(400$ 
$\left.\mathrm{MHz}, \mathrm{CDCl}_{3}\right) \delta 8.07(\mathrm{~d}, J=8.0 \mathrm{~Hz}, 2 \mathrm{H}), 7.78-7.66(\mathrm{~m}, 6 \mathrm{H}), 7.45-7.43(\mathrm{~m}, 2 \mathrm{H}), 7.37(\mathrm{dd}, J=8.4$, $1.6 \mathrm{~Hz}, 1 \mathrm{H}), 6.41-6.35(\mathrm{~m}, 1 \mathrm{H}), 5.26-5.17(\mathrm{~m}, 2 \mathrm{H}), 4.77$ (d, J=9.2 Hz, 1H), 4.58 (d, J = 9.2 Hz, 1H), $4.22(\mathrm{~d}, J=8.4 \mathrm{~Hz}, 1 \mathrm{H}), 3.74(\mathrm{~s}, 3 \mathrm{H}) .{ }^{13} \mathrm{C} \mathrm{NMR}\left(100 \mathrm{MHz}, \mathrm{CDCl}_{3}\right) \delta 172.7,164.2,135.8,135.3$, $133.3(\mathrm{q}, J=32.7 \mathrm{~Hz}), 132.6,130.3,128.9,128.3,128.1,127.6,127.5,126.9,126.1,125.9,125.3$ (q, $J=3.7 \mathrm{~Hz}), 123.7(\mathrm{q}, J=273.6 \mathrm{~Hz}), 118.8,82.3,72.3,56.5,52.7 .{ }^{19} \mathrm{~F} \mathrm{NMR}\left(376 \mathrm{MHz}, \mathrm{CDCl}_{3}\right) \delta$ -62.97. HRMS (ESI-TOF) Calcd. For $\mathrm{C}_{25} \mathrm{H}_{20} \mathrm{~F}_{3} \mathrm{NO}_{3}\left([\mathrm{M}+\mathrm{H}]^{+}\right)$: 440.1468, found: 440.1461.The product was analyzed by HPLC to determine the enantiomeric excess: 94\% ee (Chiralpak ID-H, $i$-propanol/hexane $=2 / 98$, flow rate $1.0 \mathrm{~mL} / \mathrm{min}, \lambda=254 \mathrm{~nm}) ; \mathrm{t}_{\mathrm{r}}=7.60$ and $12.14 \mathrm{~min}$.

Methyl

(S)-4-((R)-1-(furan-2-yl)

allyl)-2-(4-(trifluoromethyl)

phenyl)-4,5-dihydrooxazole-4-carboxylate (3hl):

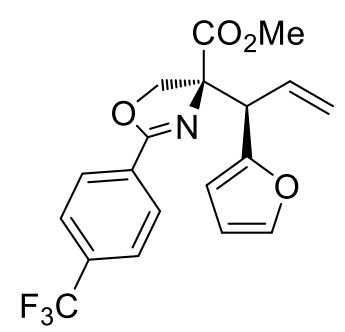

Yield (86\%); $65.1 \mathrm{mg}$; white solid; $\mathrm{mp} 53-55^{\circ} \mathrm{C}$; it was purified by column chromatography (silica gel, eluent: petroleum ether: ethyl acetate $=30: 1) .[\alpha]^{20} \mathrm{D}=+181.69\left(c 0.8, \mathrm{CHCl}_{3}\right) ;{ }^{1} \mathrm{H}$ NMR $(400$ $\left.\mathrm{MHz}, \mathrm{CDCl}_{3}\right) \delta 8.08(\mathrm{~d}, J=8.0 \mathrm{~Hz}, 2 \mathrm{H}), 7.66(\mathrm{~d}, J=8.0 \mathrm{~Hz}, 2 \mathrm{H}), 7.34-7.33(\mathrm{~m}, 1 \mathrm{H}), 6.29-6.28(\mathrm{~m}$, $1 \mathrm{H}), 6.10(\mathrm{~d}, J=3.2 \mathrm{~Hz}, 1 \mathrm{H}), 6.01-5.92(\mathrm{~m}, 1 \mathrm{H}), 5.23-5.14(\mathrm{~m}, 2 \mathrm{H}), 4.90(\mathrm{~d}, J=9.6 \mathrm{~Hz}, 1 \mathrm{H}), 4.65(\mathrm{~d}$, $J=9.2 \mathrm{~Hz}, 1 \mathrm{H}), 4.36(\mathrm{~d}, J=8.0 \mathrm{~Hz}, 1 \mathrm{H}), 3.76(\mathrm{~s}, 3 \mathrm{H}) .{ }^{13} \mathrm{C} \mathrm{NMR}\left(100 \mathrm{MHz}, \mathrm{CDCl}_{3}\right) \delta 171.6,164.2$, 151.6, 142.3, $133.4(\mathrm{q}, J=32.7 \mathrm{~Hz}), 132.4,130.3,129.0,125.3(\mathrm{q}, J=3.8 \mathrm{~Hz}), 123.7$ (q, $J=273.6$ $\mathrm{Hz}), 120.3,110.3,108.0,81.1,71.4,52.9,49.0 .{ }^{19} \mathrm{~F}$ NMR (376 $\left.\mathrm{MHz}, \mathrm{CDCl}_{3}\right) \delta$-63.03. HRMS (ESI-TOF) Calcd. For $\mathrm{C}_{19} \mathrm{H}_{16} \mathrm{~F}_{3} \mathrm{NO}_{4}\left([\mathrm{M}+\mathrm{H}]^{+}\right)$: 380.1104, found: 380.1109 . The product was analyzed by HPLC to determine the enantiomeric excess: $>99 \%$ ee (Chiralpak ID-H, $i$-propanol/hexane $=2 / 98$, flow rate $1.0 \mathrm{~mL} / \mathrm{min}, \lambda=254 \mathrm{~nm}) ; \mathrm{t}_{\mathrm{r}}=5.84$ and $6.59 \mathrm{~min}$.

Methyl (R)-2-phenyl-4-((S)-1-(p-tolyl)allyl)-4,5-dihydrothiazole-4-carboxylate (3ib): 


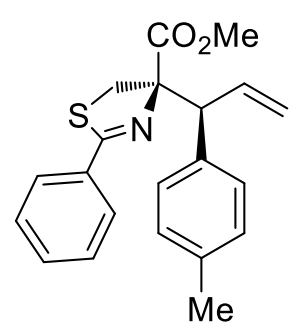

Yield (96\%); $67.4 \mathrm{mg}$; yellow oil; it was purified by column chromatography (silica gel, eluent: petroleum ether: ethyl acetate $=25: 1) .[\alpha]^{20} \mathrm{D}=+152.7\left(c 1.1, \mathrm{CHCl}_{3}\right) ;{ }^{1} \mathrm{H} \mathrm{NMR}\left(400 \mathrm{MHz}, \mathrm{CDCl}_{3}\right) \delta$ 7.89-7.87 (m, 2H), 7.51-7.46 (m, 1H), 7.46-7.39 (m, 2H), $7.16(\mathrm{~d}, J=8.4 \mathrm{~Hz}, 2 \mathrm{H}), 7.07(\mathrm{~d}, J=8.0$ $\mathrm{Hz}, 2 \mathrm{H}), 6.50-6.41(\mathrm{~m}, 1 \mathrm{H}), 5.19-5.14(\mathrm{~m}, 2 \mathrm{H}), 4.07(\mathrm{~d}, J=8.8 \mathrm{~Hz}, 1 \mathrm{H}), 3.82(\mathrm{~d}, J=11.6 \mathrm{~Hz}, 1 \mathrm{H})$, $3.73(\mathrm{~s}, 3 \mathrm{H}), 3.50(\mathrm{~d}, J=11.6 \mathrm{~Hz}, 1 \mathrm{H}), 2.29(\mathrm{~s}, 3 \mathrm{H}) .{ }^{13} \mathrm{C} \mathrm{NMR}\left(100 \mathrm{MHz}, \mathrm{CDCl}_{3}\right) \delta$ 173.3, 169.7, 137.4, 136.8, 135.4, 133.0, 131.4, 129.3, 129.1, 128.5, 128.4, 117.5, 92.5, 57.3, 52.6, 38.7, 21.0. HRMS (ESI-TOF) Calcd. For $\mathrm{C}_{21} \mathrm{H}_{21} \mathrm{NO}_{2} \mathrm{~S}\left([\mathrm{M}+\mathrm{H}]^{+}\right)$: 352.1366 , found: 352.1362 . The product was analyzed by HPLC to determine the enantiomeric excess: $>99 \%$ ee (Chiralpak ID-H, $i$-propanol/hexane $=2 / 98$, flow rate $1.0 \mathrm{~mL} / \mathrm{min}, \lambda=254 \mathrm{~nm}$ ); $\mathrm{t}_{\mathrm{r}}=5.95$ and $6.71 \mathrm{~min}$.

Methyl (R)-4-((S)-1-(4-chlorophenyl) allyl)-2-phenyl-4,5-dihydrothiazole -4-carboxylate (3ic):

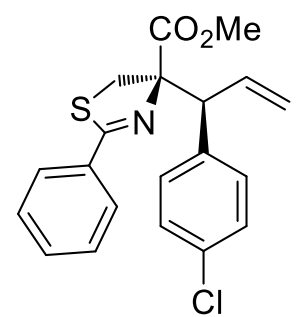

Yield (79\%); $58.6 \mathrm{mg}$; yellow oil; it was purified by column chromatography (silica gel, eluent: petroleum ether: ethyl acetate $=25: 1) .[\alpha]^{20}{ }_{\mathrm{D}}=+147.3\left(c\right.$ 0.9, $\left.\mathrm{CHCl}_{3}\right) ;{ }^{1} \mathrm{H} \mathrm{NMR}\left(400 \mathrm{MHz}, \mathrm{CDCl}_{3}\right) \delta$ 7.88-7.85 (m, 2H), 7.52-7.47 (m, 1H), 7.45-7.39 (m, 2H), 7.22-7.21 (m, 4H), 6.45-6.38 (m, 1H), 5.21-5.14 (m, 2H), $4.04(\mathrm{~d}, J=8.8 \mathrm{~Hz}, 1 \mathrm{H}), 3.79(\mathrm{~d}, J=11.6 \mathrm{~Hz}, 1 \mathrm{H}), 3.73(\mathrm{~s}, 3 \mathrm{H}), 3.42(\mathrm{~d}, J=11.6$ $\mathrm{Hz}, 1 \mathrm{H}) .{ }^{13} \mathrm{C}$ NMR $\left(100 \mathrm{MHz}, \mathrm{CDCl}_{3}\right) \delta 173.2,170.2,137.1,137.0,133.1,132.8,131.6,130.8$, 128.5, 118.1, 92.1, 57.1, 52.7, 39.0, HRMS (ESI-TOF) Calcd. For $\mathrm{C}_{20} \mathrm{H}_{18} \mathrm{ClNO}_{2} \mathrm{~S}\left([\mathrm{M}+\mathrm{H}]^{+}\right)$: 372.0820, found: 372.0815. The product was analyzed by HPLC to determine the enantiomeric excess: $>99 \%$ ee (Chiralpak ID-H, $i$-propanol/hexane $=2 / 98$, flow rate $1.0 \mathrm{~mL} / \mathrm{min}, \lambda=254 \mathrm{~nm}) ; \mathrm{t}_{\mathrm{r}}=$ 5.64 and $6.75 \mathrm{~min}$. 
Methyl (R)-4-((S)-1-(3-chlorophenyl) allyl)-2-phenyl-4,5-dihydrothiazole-4-carboxylate (3ig):

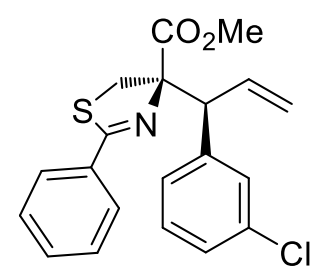

Yield (94\%); $69.8 \mathrm{mg}$; yellow oil; it was purified by column chromatography (silica gel, eluent: petroleum ether: ethyl acetate $=25: 1) .[\alpha]^{20}{ }_{\mathrm{D}}=+137.8\left(c 1.2, \mathrm{CHCl}_{3}\right) ;{ }^{1} \mathrm{H} \mathrm{NMR}\left(400 \mathrm{MHz}, \mathrm{CDCl}_{3}\right) \delta$ 7.87-7.85 (m, 2H), 7.51-7.47 (m, 1H), 7.44-7.40 (m, 2H), 7.29-7.28 (m, 1H), 7.21-7.15 (m, 3H), 6.47-6.38 (m, 1H), 5.23-5.17 (m, 2H), $4.03(\mathrm{~d}, J=8.8 \mathrm{~Hz}, 1 \mathrm{H}), 3.80(\mathrm{~d}, J=11.6 \mathrm{~Hz}, 1 \mathrm{H}), 3.73(\mathrm{~s}$, $3 \mathrm{H}), 3.44(\mathrm{~d}, J=11.6 \mathrm{~Hz}, 1 \mathrm{H}) .{ }^{13} \mathrm{C} \mathrm{NMR}\left(100 \mathrm{MHz}, \mathrm{CDCl}_{3}\right) \delta 173.0,170.3,140.5136 .6,134.0$, $132.8,131.6,129.7,129.5,128.44,128.43,127.6,127.4,118.3,92.0,57.4,52.7,39.0$. HRMS (ESI-TOF) Calcd. For $\mathrm{C}_{20} \mathrm{H}_{18} \mathrm{ClNO}_{2} \mathrm{~S}\left([\mathrm{M}+\mathrm{H}]^{+}\right)$: 372.0820, found: 372.0816. The product was analyzed by HPLC to determine the enantiomeric excess: $>99 \%$ ee (Chiralpak ID-H, $i$-propanol $/$ hexane $=2 / 98$, flow rate $1.0 \mathrm{~mL} / \mathrm{min}, \lambda=254 \mathrm{~nm}) ; \mathrm{t}_{\mathrm{r}}=6.61$ and $7.20 \mathrm{~min}$.

\section{Methyl (R)-4-((R)-1-(furan-2-yl) allyl)-2-phenyl-4,5-dihydrothiazole-4-carboxylate (3il):}

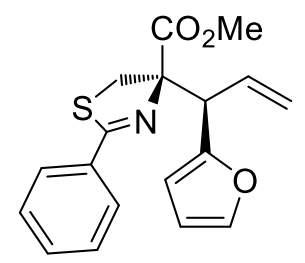

Yield (94\%); $64.5 \mathrm{mg}$; yellow oil; it was purified by column chromatography (silica gel, eluent: petroleum ether: ethyl acetate $=25: 1) .[\alpha]^{20} \mathrm{D}=+131.0\left(c 1.4, \mathrm{CHCl}_{3}\right) ;{ }^{1} \mathrm{H} \mathrm{NMR}\left(400 \mathrm{MHz}, \mathrm{CDCl}_{3}\right) \delta$ 7.86-7.83 (m, 2H), 7.49-7.44 (m, 1H), 7.42-7.37 (m, 2H), 7.34-7.33 (m, 1H), 6.29-6.28 (m, 1H), 6.16-6.08 (m, 2H), 5.22-5.18 (m, 2H), $4.45(\mathrm{~d}, J=8.4 \mathrm{~Hz}, 1 \mathrm{H}), 3.96(\mathrm{~d}, J=11.6 \mathrm{~Hz}, 1 \mathrm{H}), 3.76$ (s, $3 \mathrm{H}), 3.62(\mathrm{~d}, J=11.6 \mathrm{~Hz}, 1 \mathrm{H}) .{ }^{13} \mathrm{C} \mathrm{NMR}\left(100 \mathrm{MHz}, \mathrm{CDCl}_{3}\right) \delta 172.1,170.1,152.0,142.0,133.4$, 132.8, 131.5, 128.5, 128.4, 119.2, 110.4, 108.1, 91.9, 52.9, 50.9, 36.8. HRMS (ESI-TOF) Calcd. For $\mathrm{C}_{18} \mathrm{H}_{17} \mathrm{NO}_{3} \mathrm{~S}\left([\mathrm{M}+\mathrm{H}]^{+}\right)$: 328.1002, found: 328.0997. The product was analyzed by HPLC to determine the enantiomeric excess: >99\% ee (Chiralpak ID-H, $i$-propanol/ hexane $=2 / 98$, flow rate $1.0 \mathrm{~mL} / \mathrm{min}, \lambda=254 \mathrm{~nm}) ; \mathrm{t}_{\mathrm{r}}=6.78$ and $7.60 \mathrm{~min}$.

Methyl (R)-4-((R)-but-3-en-2-yl)-2-phenyl-4,5-dihydrothiazole-4-carboxylate (3im): 


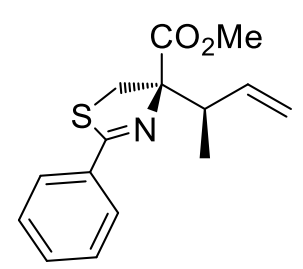

Yield (88\%); $48.4 \mathrm{mg}$; yellow oil; it was purified by column chromatography (silica gel, eluent: petroleum ether: ethyl acetate $=30: 1) .[\alpha]^{20} \mathrm{D}=+43.5\left(c 0.7, \mathrm{CHCl}_{3}\right) ;{ }^{1} \mathrm{H} \mathrm{NMR}\left(400 \mathrm{MHz}, \mathrm{CDCl}_{3}\right) \delta$ 7.88-7.85 (m, 2H), 7.47-7.38 (m, 3H), 5.95-5.86 (m, 1H), 5.16-5.10 (m, 2H), $3.90(\mathrm{~d}, J=11.6 \mathrm{~Hz}$, 1H), $3.79(\mathrm{~s}, 3 \mathrm{H}), 3.38(\mathrm{~d}, J=11.6 \mathrm{~Hz}, 1 \mathrm{H}), 3.13-3.06(\mathrm{~m}, 1 \mathrm{H}), 1.09(\mathrm{~d}, J=7.2 \mathrm{~Hz}, 3 \mathrm{H}) .{ }^{13} \mathrm{C} \mathrm{NMR}$ $\left(100 \mathrm{MHz}, \mathrm{CDCl}_{3}\right) \delta 172.8,168.6,138.3,132.9,131.4,128.5,128.4,116.6,92.4,52.7,45.1,36.6$, 14.3, HRMS (ESI-TOF) Calcd. For $\mathrm{C}_{15} \mathrm{H}_{17} \mathrm{NO}_{2} \mathrm{~S}\left([\mathrm{M}+\mathrm{H}]^{+}\right)$: 276.1053, found: 276.1046. The product was analyzed by HPLC to determine the enantiomeric excess: $>99 \%$ ee (Chiralcel OD-H, $i$-propanol $/$ hexane $=1 / 99$, flow rate $1.0 \mathrm{~mL} / \mathrm{min}, \lambda=254 \mathrm{~nm}) ; \mathrm{t}_{\mathrm{r}}=5.39$ and $6.97 \mathrm{~min}$.

\section{Gram Scale Reaction and Synthetic Transformation}
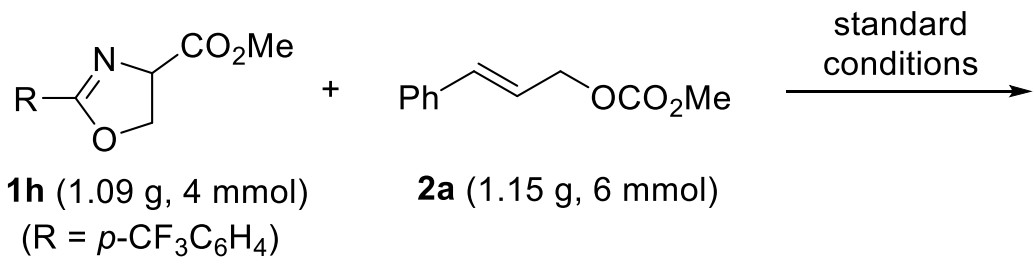

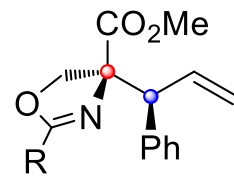

$3 \mathrm{ha},>20: 1 \mathrm{dr}$ $90 \%$ yield, $99 \%$ ee

A flame dried Schlenk tube was cooled to $\mathrm{rt}$ and filled with $\mathrm{N}_{2}$. To this flask were added $[\operatorname{Ir}(\mathrm{COD}) \mathrm{Cl}]_{2}(0.05 \mathrm{~mol}, 1.25 \mathrm{~mol} \%)$, phosphoramidite ligand $(S, S, S)-\mathbf{L 5}(0.1 \mathrm{~mol}, 2.5 \mathrm{~mol} \%)$, degassed THF $(2 \mathrm{~mL})$ and degassed $n$-propylamine $(2 \mathrm{~mL})$. The reaction mixture was heated at $50{ }^{\circ} \mathrm{C}$ in oil bath for $30 \mathrm{~min}$ and then the volatile solvents were removed under vacuum to give a pale-yellow solid. Meanwhile, in a separated Schlenk tube, $\left(R, R_{p}\right){ }^{i}$ Pr-Phosferrox-L1 $(0.22 \mathrm{mmol}, 5.5$ mol \%) and $\mathrm{Cu}(\mathrm{MeCN})_{4} \mathrm{BF}_{4}(0.2 \mathrm{mmol}, 5 \mathrm{~mol} \%)$ were dissolved in $4 \mathrm{~mL}$ of $\mathrm{THF}$, and stirred at room temperature for about $0.5 \mathrm{~h}$. The $\mathrm{Cu} / \mathrm{L} \mathbf{1}$ complex solution was then transformed to the Schlenk tube containing iridium complex via syringe. Allylic carbonates 2a $(6$ mmol), 2-oxazoline-4-carboxylate $\mathbf{1 h}(4 \mathrm{mmol}), \mathrm{Et}_{3} \mathrm{~N}(4 \mathrm{mmol})$ and $\mathrm{THF}(6 \mathrm{~mL})$ were then added. Once starting material was consumed (monitored by TLC), the organic solvent was removed by rotary 
evaporation and the residue was purified by column chromatography to give the allylation product 3ha $(1.40 \mathrm{~g}, 90 \%)$.

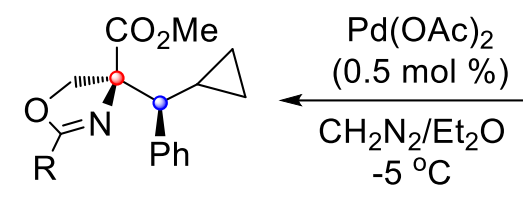

$4,75 \%$ yield $98 \%$ ee

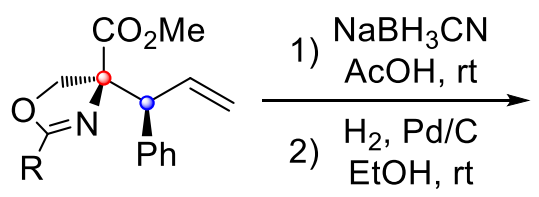

3ha $99 \%$ ee<smiles>CCC(=O)[C@](N)(CO)C(=O)OC</smiles>

$5,60 \%$ yield $99 \%$ ee

3ha $(78 \mathrm{mg}, 0.2 \mathrm{mmol}$ ) was added to a dry solution of diazomethane (prepare from $0.1 \mathrm{~g}$ $N$-methyl- $N$-nitrosourea, $2 \mathrm{~mL}$ ethyl ether) at $-5{ }^{\circ} \mathrm{C}$. Then $\mathrm{Pd}(\mathrm{OAc})_{2}(0.22 \mathrm{mg}, 0.01 \mathrm{mmol})$ was added in one portion and gas evolution was observed. After $1 \mathrm{~h}$ vigorous stirring at $-5{ }^{\circ} \mathrm{C}$, the residue was filtered by Buchner funnel and the organic solvent was removed by rotary evaporation and then purified by column chromatography to give the desired product 4 (75\% yield).

\section{Methyl (S)-4-((S)-cyclopropyl(phenyl)methyl)-2-(4-(trifluoromethyl)phenyl)-4,5-dihydro} oxazole-4-carboxylate (4):<smiles>CC(=O)[C@](CO)(N=C(O)c1ccc(C(F)(F)F)cc1)C(c1ccccc1)C1CC1</smiles>

Yield (75\%); white solid; mp $80-82{ }^{\circ} \mathrm{C}$; it was purified by column chromatography (silica gel, eluent: petroleum ether: ethyl acetate $=15: 1) .[\alpha]^{20} \mathrm{D}=+84.9\left(c 0.8, \mathrm{CHCl}_{3}\right) ;{ }^{1} \mathrm{H} \mathrm{NMR}\left(400 \mathrm{MHz}, \mathrm{CDCl}_{3}\right) \delta$ $7.96(\mathrm{~d}, J=8.0 \mathrm{~Hz}, 2 \mathrm{H}), 7.62(\mathrm{~d}, J=8.0 \mathrm{~Hz}, 2 \mathrm{H}), 7.35-7.33(\mathrm{~m}, 2 \mathrm{H}), 7.22-7.15(\mathrm{~m}, 3 \mathrm{H}), 4.88(\mathrm{~d}, J=$ $9.2 \mathrm{~Hz}, 1 \mathrm{H}), 4.62(\mathrm{~d}, J=9.2 \mathrm{~Hz}, 1 \mathrm{H}), 3.79(\mathrm{~s}, 3 \mathrm{H}), 2.74(\mathrm{~d}, J=10.0 \mathrm{~Hz}, 1 \mathrm{H}), 1.07-1.01(\mathrm{~m}, 1 \mathrm{H})$, 0.74-0.69 (m, 1H), 0.59-0.52 (m, 1H), 0.45-0.39 (m, 1H), 0.08-0.01 (m, 1H). ${ }^{13} \mathrm{C}$ NMR (100 MHz, $\left.\mathrm{CDCl}_{3}\right) \delta 172.7,163.2,138.9,133.1(\mathrm{q}, J=35.7 \mathrm{~Hz}), 130.5129 .5,128.8,127.6,127.0,125.2(\mathrm{q}, J=$ $3.7 \mathrm{~Hz}), 123.7(\mathrm{q}, J=273.5 \mathrm{~Hz}), 82.2,70.9,55.5,52.5,11.0,7.7,3.4 .{ }^{19} \mathrm{~F} \mathrm{NMR}\left(376 \mathrm{MHz}, \mathrm{CDCl}_{3}\right) \delta$ -63.00. HRMS (ESI-TOF) Calcd. For $\mathrm{C}_{22} \mathrm{H}_{20} \mathrm{~F}_{3} \mathrm{NO}_{3}\left([\mathrm{M}+\mathrm{H}]^{+}\right)$: 404.1468, found: 404.1461. The product was analyzed by HPLC to determine the enantiomeric excess: 98\% ee (Chiralpak ID-H, 
$i$-propanol/hexane $=5 / 95$, flow rate $1.0 \mathrm{~mL} / \mathrm{min}, \lambda=254 \mathrm{~nm}) ; \mathrm{t}_{\mathrm{r}}=5.32$ and $6.98 \mathrm{~min}$.

To a solution of $\mathrm{NaBH}_{3} \mathrm{CN}(37.7 \mathrm{mg}, 0.6 \mathrm{mmol})$ in $\mathrm{AcOH}(3.5 \mathrm{~mL})$ at $0{ }^{\circ} \mathrm{C}$ was added $3 \mathrm{ha}(194$ $\mathrm{mg}, 0.5 \mathrm{mmol})$. The mixture was stirred for $17 \mathrm{~h}$ at room temperature, the reaction solvent was then removed. The residue was partitioned between EtOAc $(3 \mathrm{~mL})$ and sat. $\mathrm{Na}_{2} \mathrm{CO}_{3}$ solution $(5 \mathrm{~mL})$. The organic layer was treated with $10 \% \mathrm{NaOH}$ until $\mathrm{pH}>12$. The aqueous layer was washed with EtOAc $(2 \times 10 \mathrm{~mL})$. The combined organic layers were dried $\left(\mathrm{Na}_{2} \mathrm{SO}_{4}\right)$ and concentrated in vacuo. The crude product was used in the next step without purification. $\mathrm{Pd} / \mathrm{C}(21 \mathrm{~g})$ was added to a solution of the obtained crude product and $\mathrm{H}_{2}$ was introduced using a $\mathrm{H}_{2}$ balloon. After stirring at $\mathrm{rt}$ for $24 \mathrm{~h}$, the mix was filtered through Celite to remove the $\mathrm{Pd} / \mathrm{C}$, washed with $\mathrm{EtOH}$ and concentrated in vacuo. The residue was purified by chromatography (silica gel, $\mathrm{CH}_{2} \mathrm{Cl}_{2}: \mathrm{MeOH}=95: 5$ with $0.1 \% \mathrm{NH}_{4} \mathrm{OH}$ ) to afford 5 (60\% yield) as a colorless liquid.

\section{Methyl (2S, 3S)-2-amino-2-(hydroxymethyl)-3-phenylpentanoate (5):}

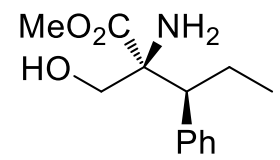

Yield (60\%); yellow oil; it was purified by column chromatography (silica gel, eluent: $\mathrm{CH}_{2} \mathrm{Cl}_{2}$ : $\mathrm{MeOH}=95: 5) .[\alpha]^{20} \mathrm{D}=-17.8\left(c 1.3, \mathrm{CHCl}_{3}\right) ;{ }^{1} \mathrm{H}$ NMR $\left(400 \mathrm{MHz}, \mathrm{CDCl}_{3}\right) \delta$ 7.30-7.21 (m, 3H), 7.14-7.12 (m, 2H), $3.90(\mathrm{~d}, J=10.4 \mathrm{~Hz}, 1 \mathrm{H}), 3.65(\mathrm{~d}, J=10.8 \mathrm{~Hz}, 1 \mathrm{H}), 3.56(\mathrm{~s}, 3 \mathrm{H}), 2.89$ (dd, $J=$ 11.6, $4.0 \mathrm{~Hz}, 1 \mathrm{H}), 2.14$ (brs, 3H), 1.83-1.75 (m, 2H), 0.72 (t, $J=7.2 \mathrm{~Hz}, 3 \mathrm{H}) .{ }^{13} \mathrm{C} \mathrm{NMR}(100 \mathrm{MHz}$, $\left.\mathrm{CDCl}_{3}\right) \delta 175.3,138.9,128.8,128.3,127.1,66.8,66.5,52.7,52.0,21.2,12.3$. HRMS (ESI-TOF) Calcd. For $\mathrm{C}_{13} \mathrm{H}_{19} \mathrm{NO}_{3}\left([\mathrm{M}+\mathrm{H}]^{+}\right)$: 238.1438 , found: 238.1435 . It was not separated well to obtain the ee value by the HPLC, this transformation wasn't involved the change of stereoselectivity. Therefore, the ee value was deduced on the basis of compound 3 ha. 


\section{Absolute Configuration Determination of $(S, S)-3$ he}
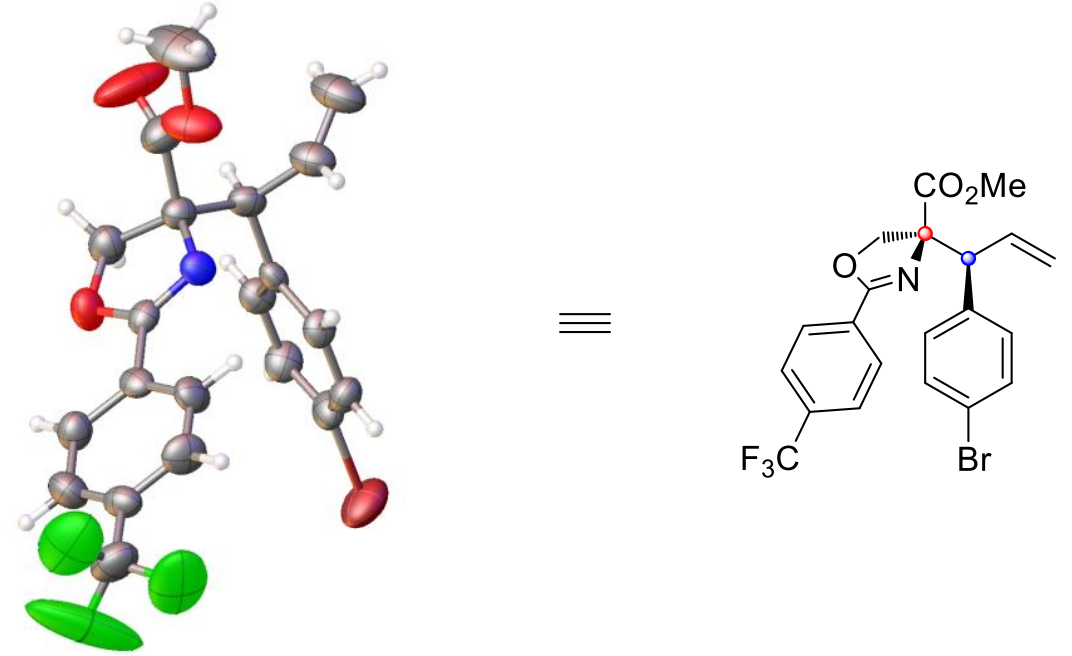

Figure S1. X-ray structure of $(S, S)$-3he at 30\% probability for the drawing of thermal ellipsoids.

To a $10 \mathrm{~mL}$ oven-dried glass sample bottle was added $10 \mathrm{mg}$ pure 3he with $0.1 \mathrm{~mL}$ EtOAc to get clear solution, then $7 \mathrm{~mL} n$-hexane was slowly added. The mixture solution was sealed with filter paper to slowly grow crystals at room temperature. Crystal data for $(S, S)-3$ he: $\mathrm{C}_{21} \mathrm{H}_{17} \mathrm{BrF}_{3} \mathrm{NO}_{3}, M_{\mathrm{r}}=$ 468.27, $T=298 \mathrm{~K}$, Monoclinic, space group P2(1)2(1)2(1), $a=10.320(2), b=17.933(4), c=$ 22.201(5) $\AA, V=4108.6(16) \AA^{3}, Z=8,4860$ unique reflections, final $R_{1}=0.0538$ and $w R_{2}=0.1205$ for 7728 observed $[I>2 \sigma(I)]$ reflections, Flack $=0.050(7)$. CCDC 1999192 contains the supplementary crystallographic data for this paper. The data can be obtained free of charge via www.ccdc.cam.ac.uk/conts/retrieving.html (or from the Cambridge Crystallographic Data Centre, 12, Union Road, Cambridge CB21EZ, UK; fax: (+44) 1223-336-033; or deposit@ ccdc.cam.ac.uk). 
V. Reference

1. Diéguez, M.; Pàmies, O. Chem. Eur. J. 2008, 14, 3653.

2. Stanley, L. M.; Hartwig, J. F. Angew. Chem. Int. Ed. 2009, 48, 7841.

3. Richards, C. J.; Mulvaney, A. W. Tetrahedron: Asymmetry 1996, 7, 1419.

4. Smith, C. R.; Mans, D. J.; Rajanbabu, T. V. Org. Synth. 2008, 85, 238. 
VI. ${ }^{1} \mathrm{H}$ NMR and ${ }^{13} \mathrm{C}$ NMR Spectra

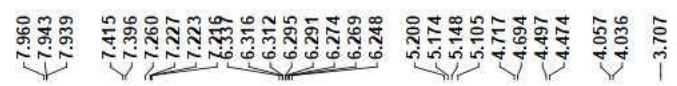
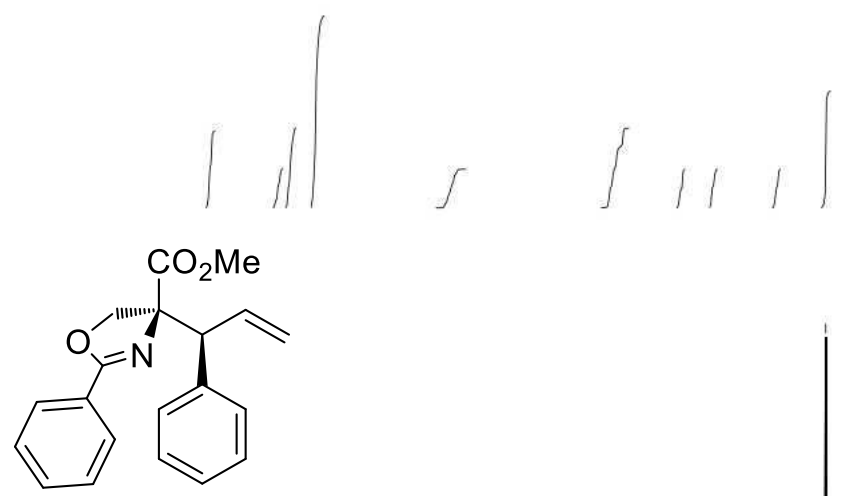

$(S, S)-3 a a$

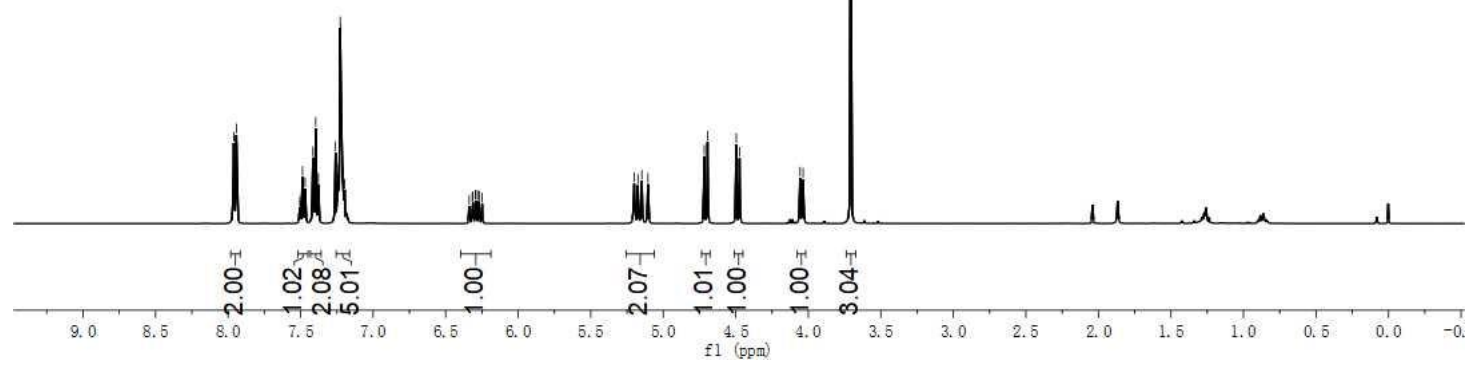

${ }^{1} \mathbf{H}$ NMR $\left(400 \mathrm{MHz}, \mathrm{CDCl}_{3}\right)$

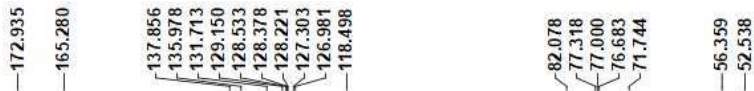

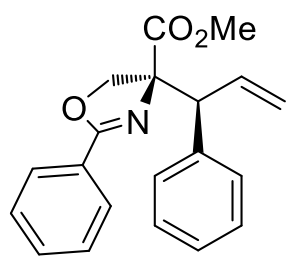

(S, S)-3aa
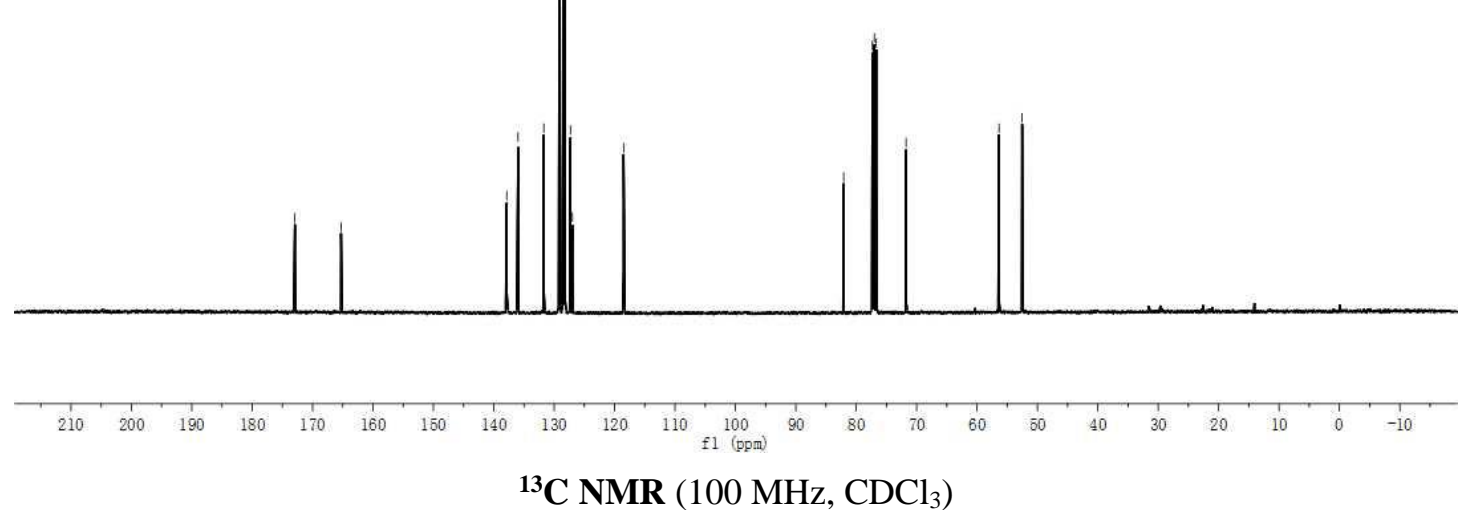


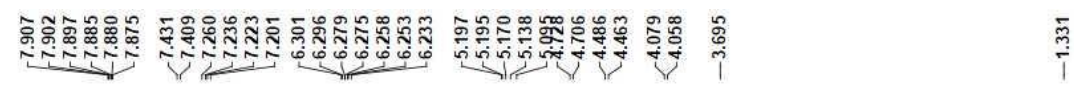

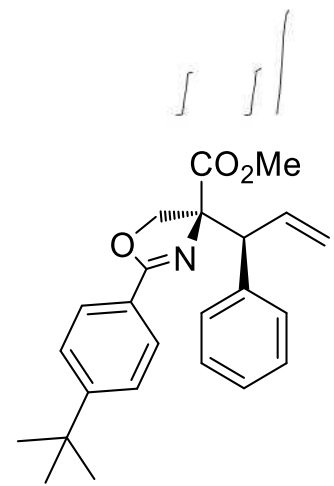

(S, S)-3ba

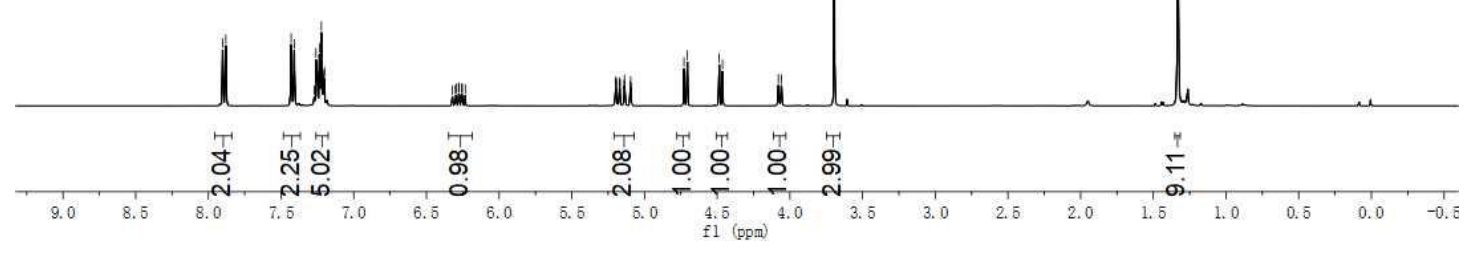

${ }^{1} \mathbf{H} \mathbf{N M R}\left(400 \mathrm{MHz}, \mathrm{CDCl}_{3}\right)$

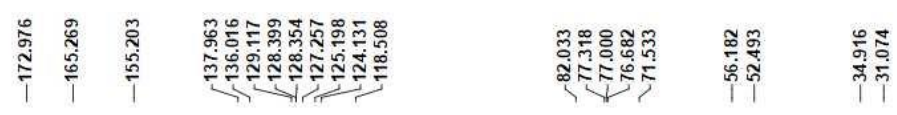

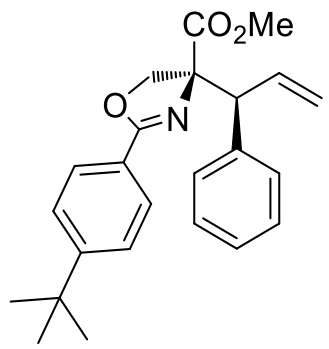

$(S, S)-3 \mathrm{ba}$
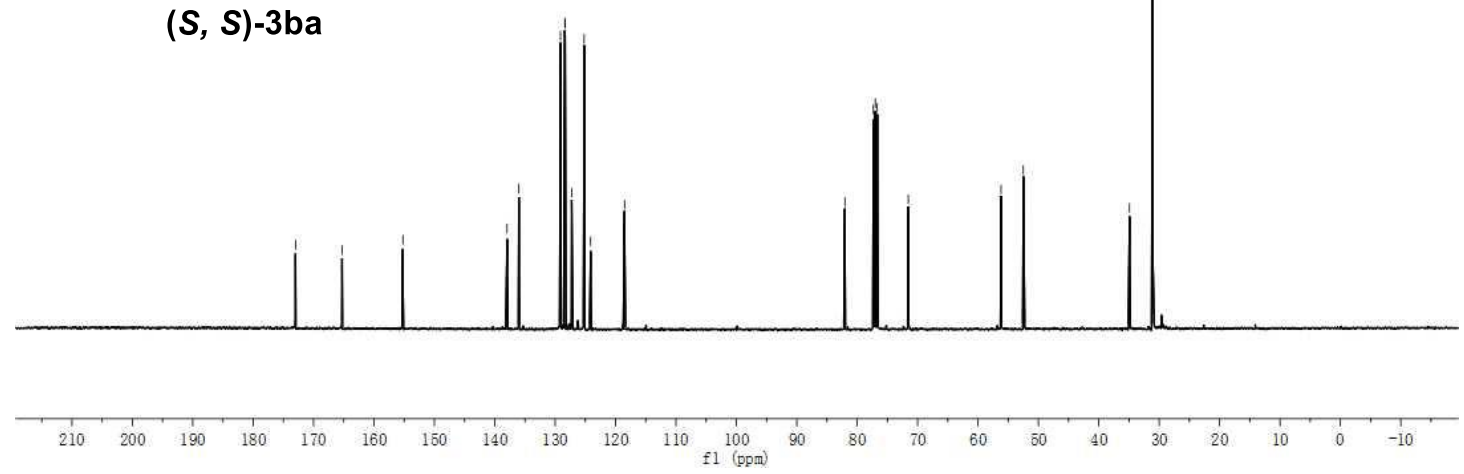

${ }^{13} \mathbf{C}$ NMR (100 MHz, $\left.\mathrm{CDCl}_{3}\right)$ 


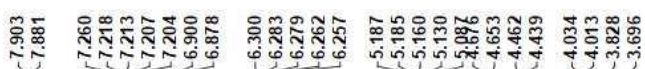

$1 \mid 1+11111$<smiles>C=CC(c1ccccc1)[C@](C)(CO)NC(=O)c1ccc(OC)cc1</smiles>

$(S, S)-3 c a$

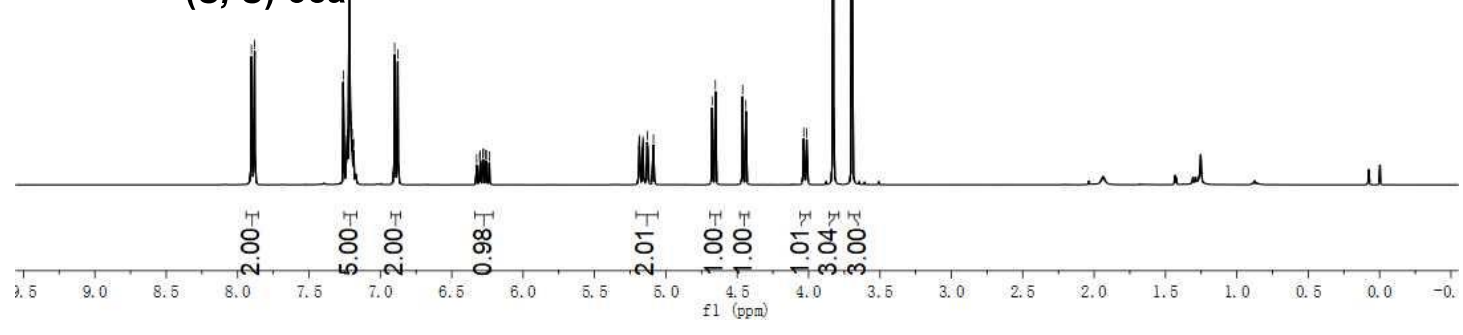

${ }^{1} \mathbf{H}$ NMR $\left(400 \mathrm{MHz}, \mathrm{CDCl}_{3}\right)$

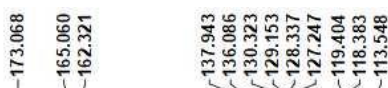

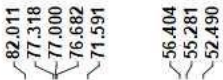

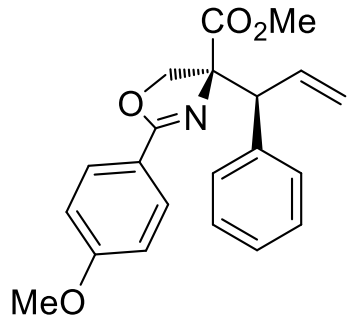

$(S, S)-3 c a$
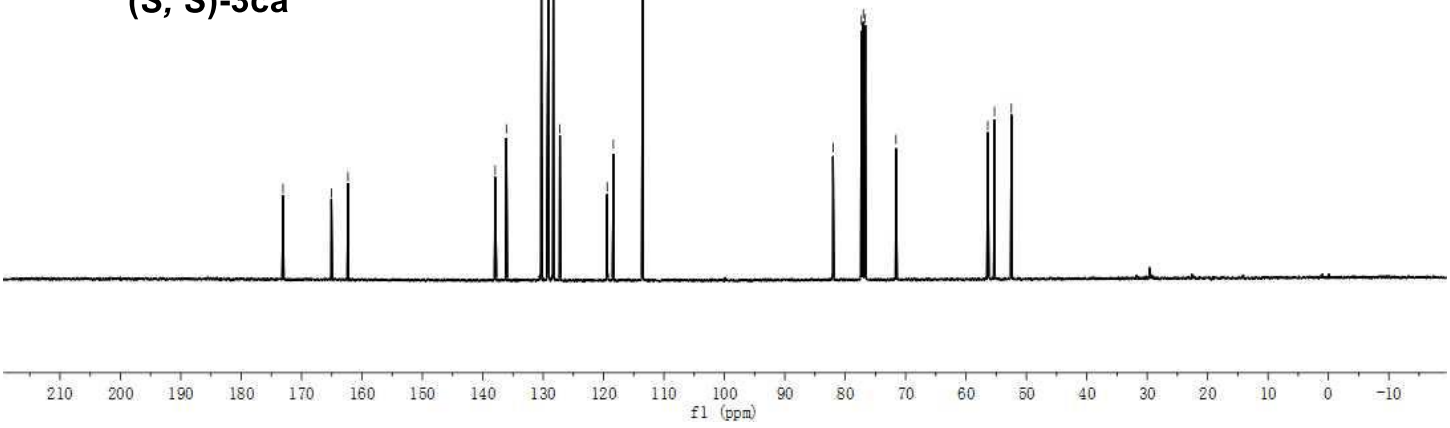

${ }^{13} \mathbf{C}$ NMR $\left(100 \mathrm{MHz}, \mathrm{CDCl}_{3}\right)$ 


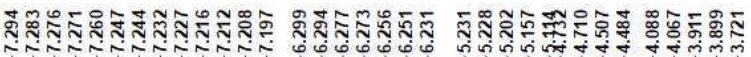

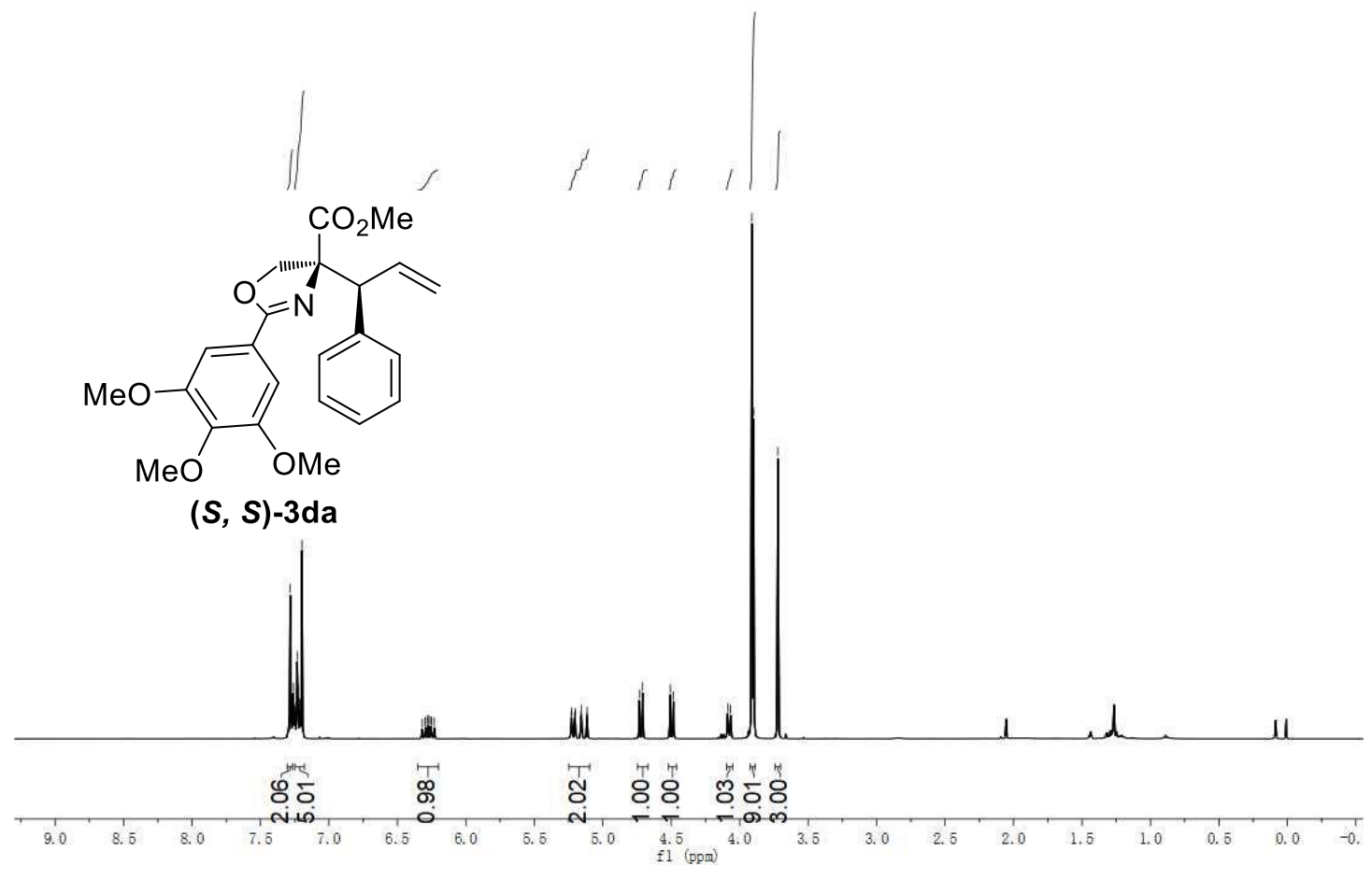

${ }^{1} \mathbf{H}$ NMR $\left(400 \mathrm{MHz}, \mathrm{CDCl}_{3}\right)$

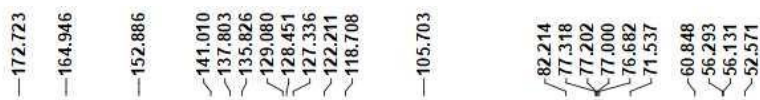

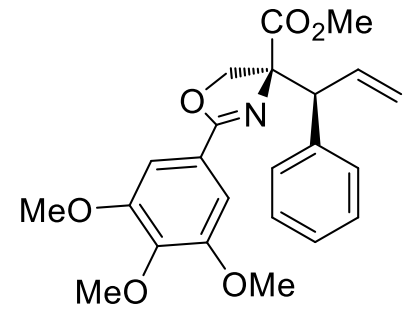

$(S, S)-3 d a$
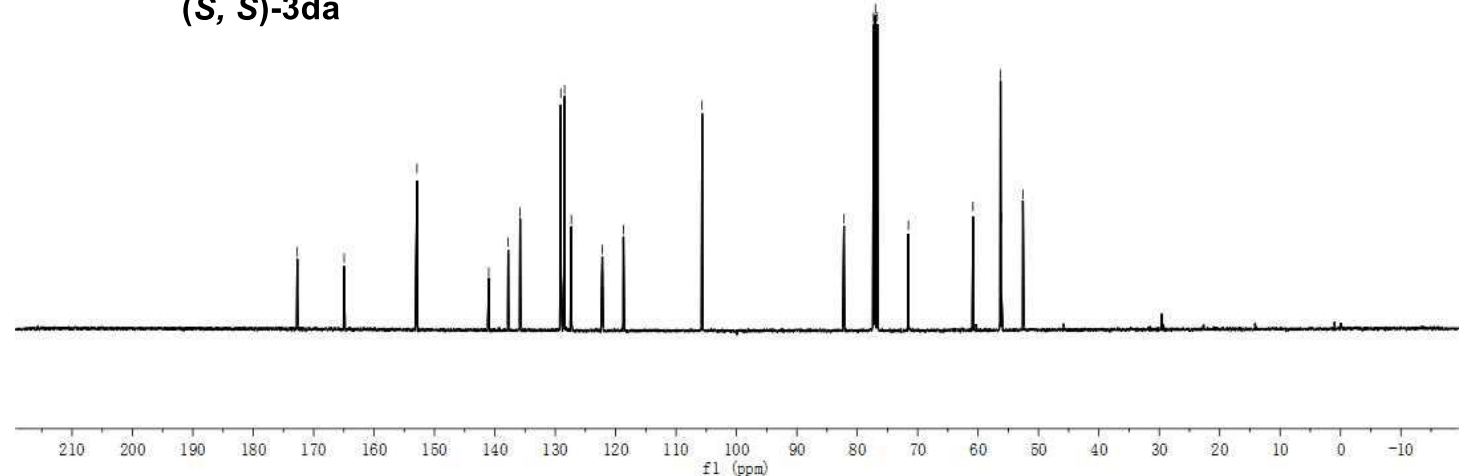

${ }^{13} \mathbf{C}$ NMR $\left(100 \mathrm{MHz}, \mathrm{CDCl}_{3}\right)$ 


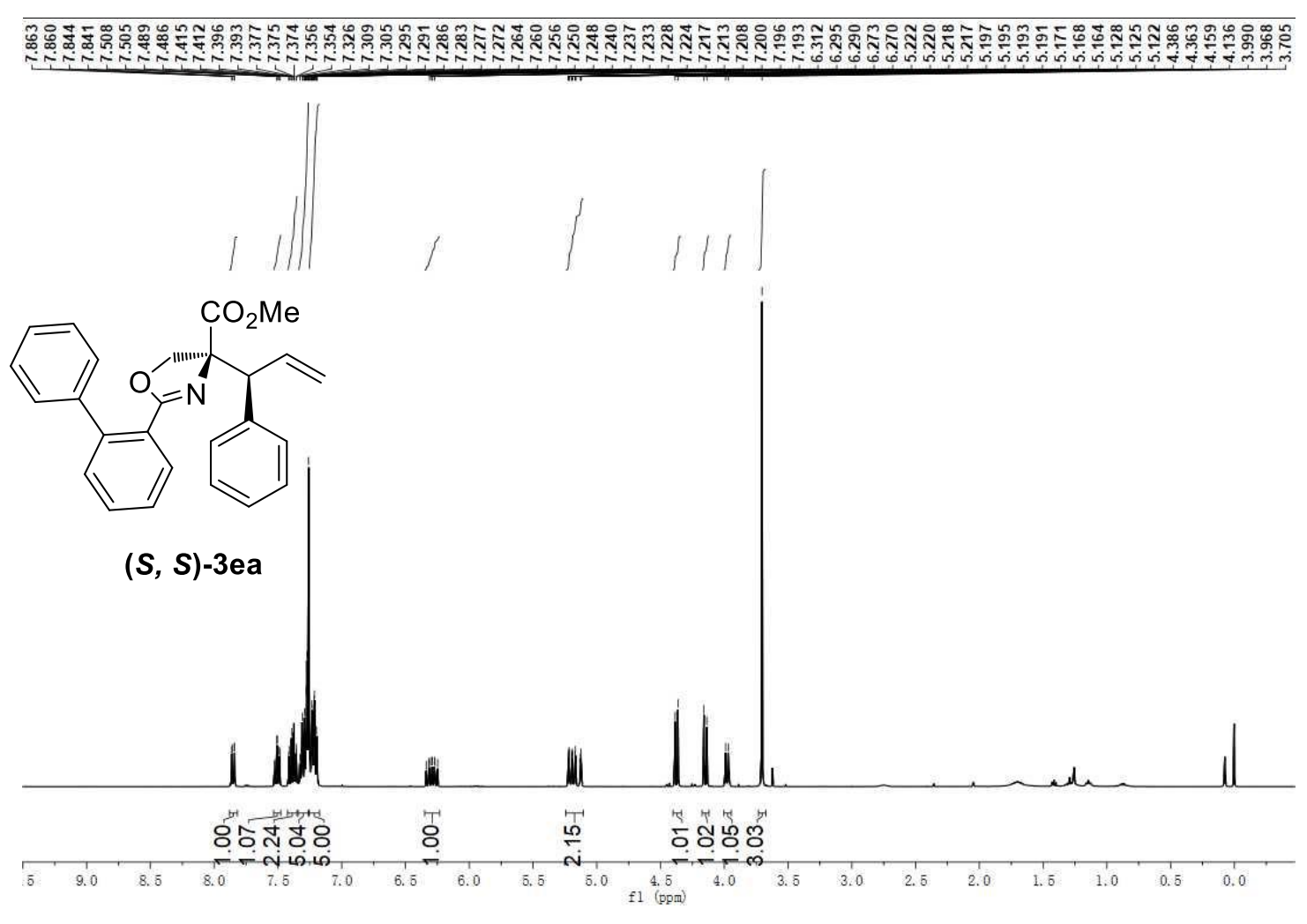

${ }^{1} \mathbf{H}$ NMR $\left(400 \mathrm{MHz}, \mathrm{CDCl}_{3}\right)$

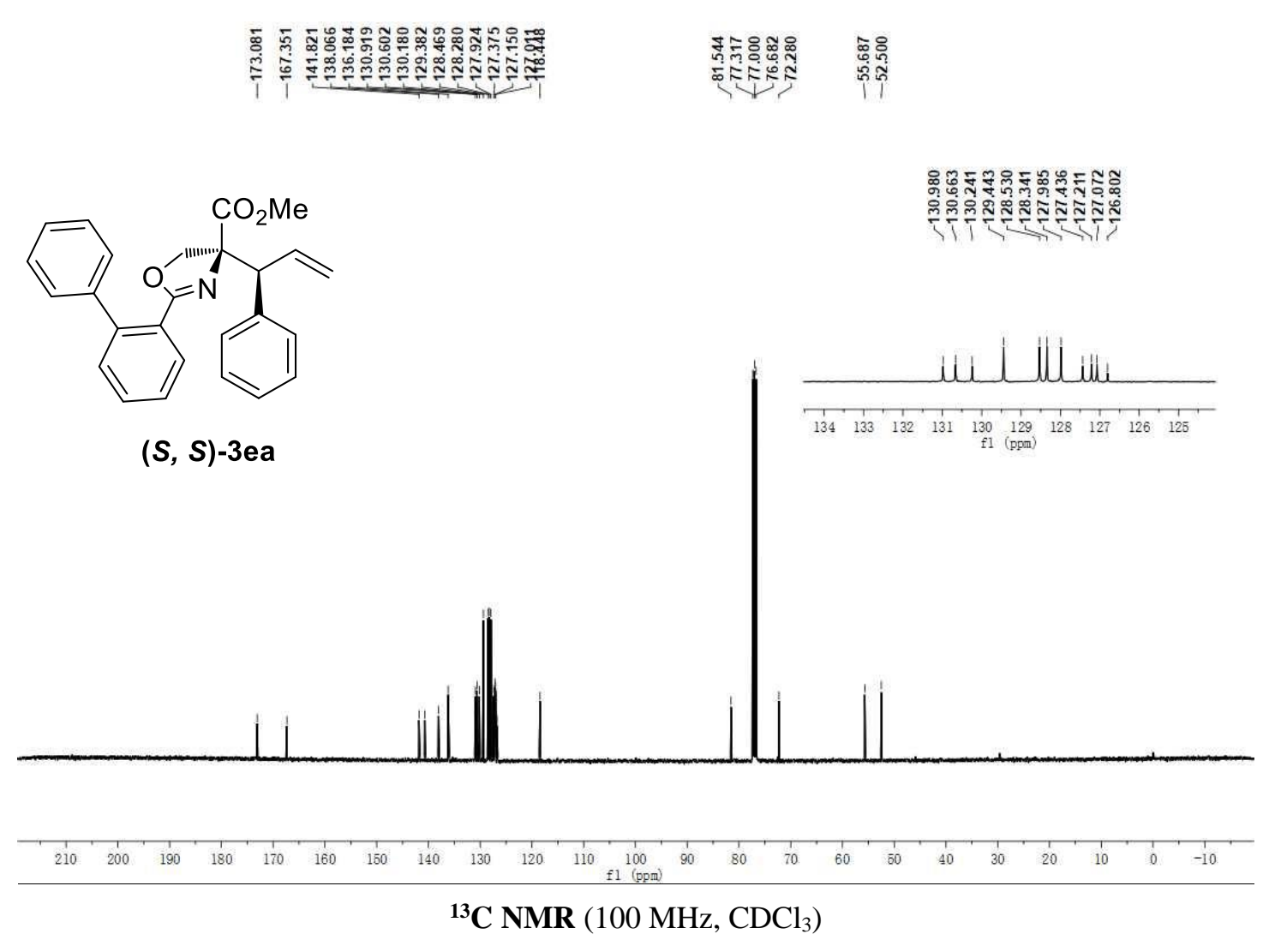




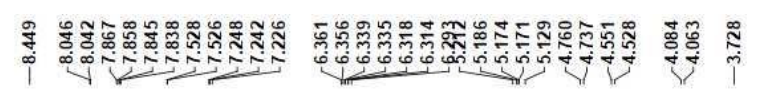
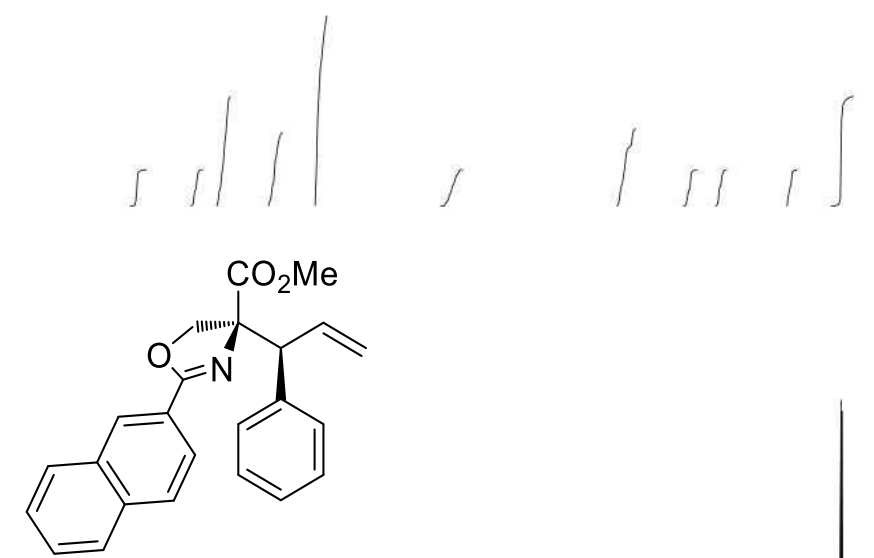

$(S, S)-3 f a$

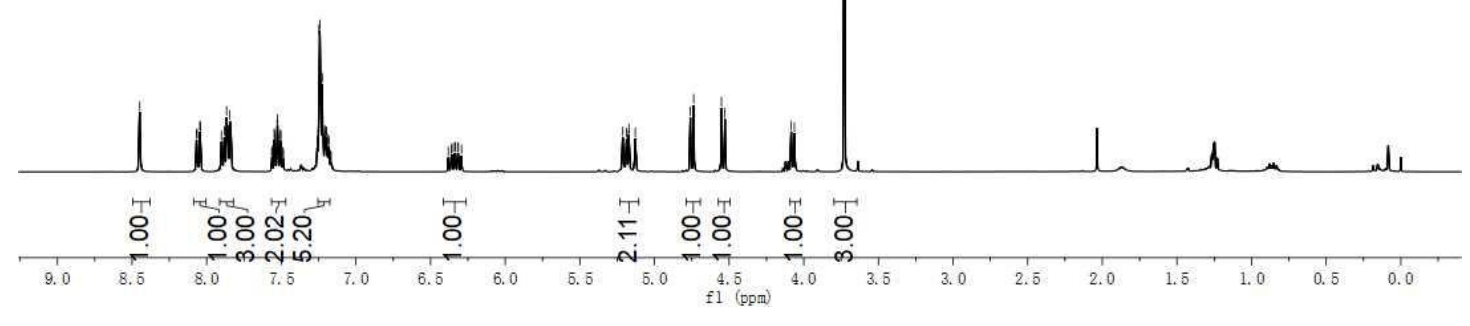

${ }^{1} \mathbf{H}$ NMR $\left(400 \mathrm{MHz}, \mathrm{CDCl}_{3}\right)$

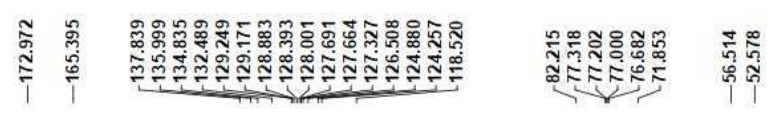
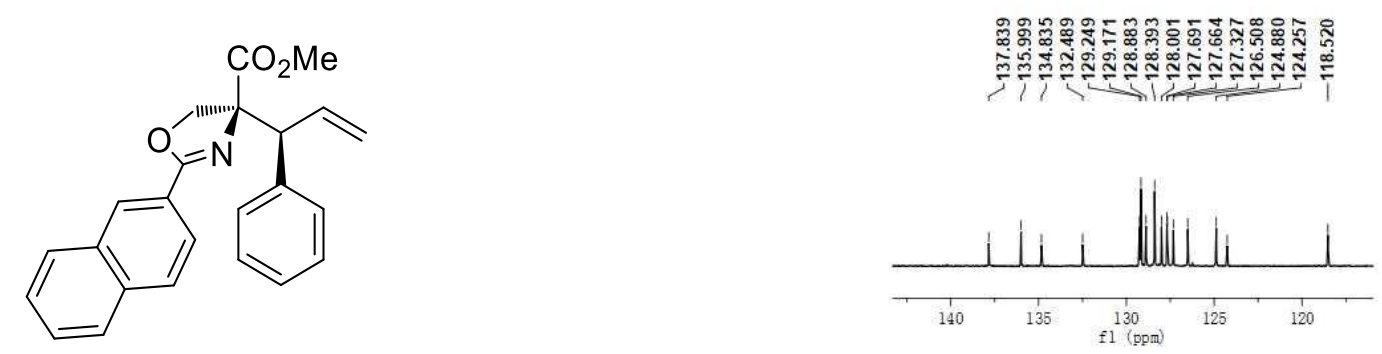

$(S, S)-3 f a$

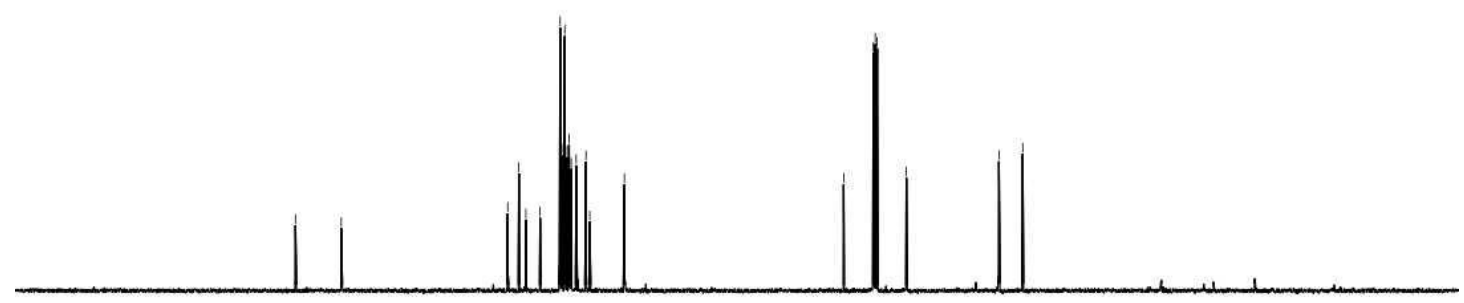

$\begin{array}{llllllllllllllllllllllllllll}210 & 200 & 190 & 180 & 170 & 160 & 150 & 140 & 130 & 120 & 110 & 100 & 90 & 80 & 70 & 60 & 50 & 40 & 30 & 20 & 10 & 0 & -10\end{array}$

${ }^{13}$ C NMR (100 MHz, $\left.\mathrm{CDCl}_{3}\right)$ 


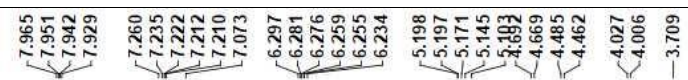
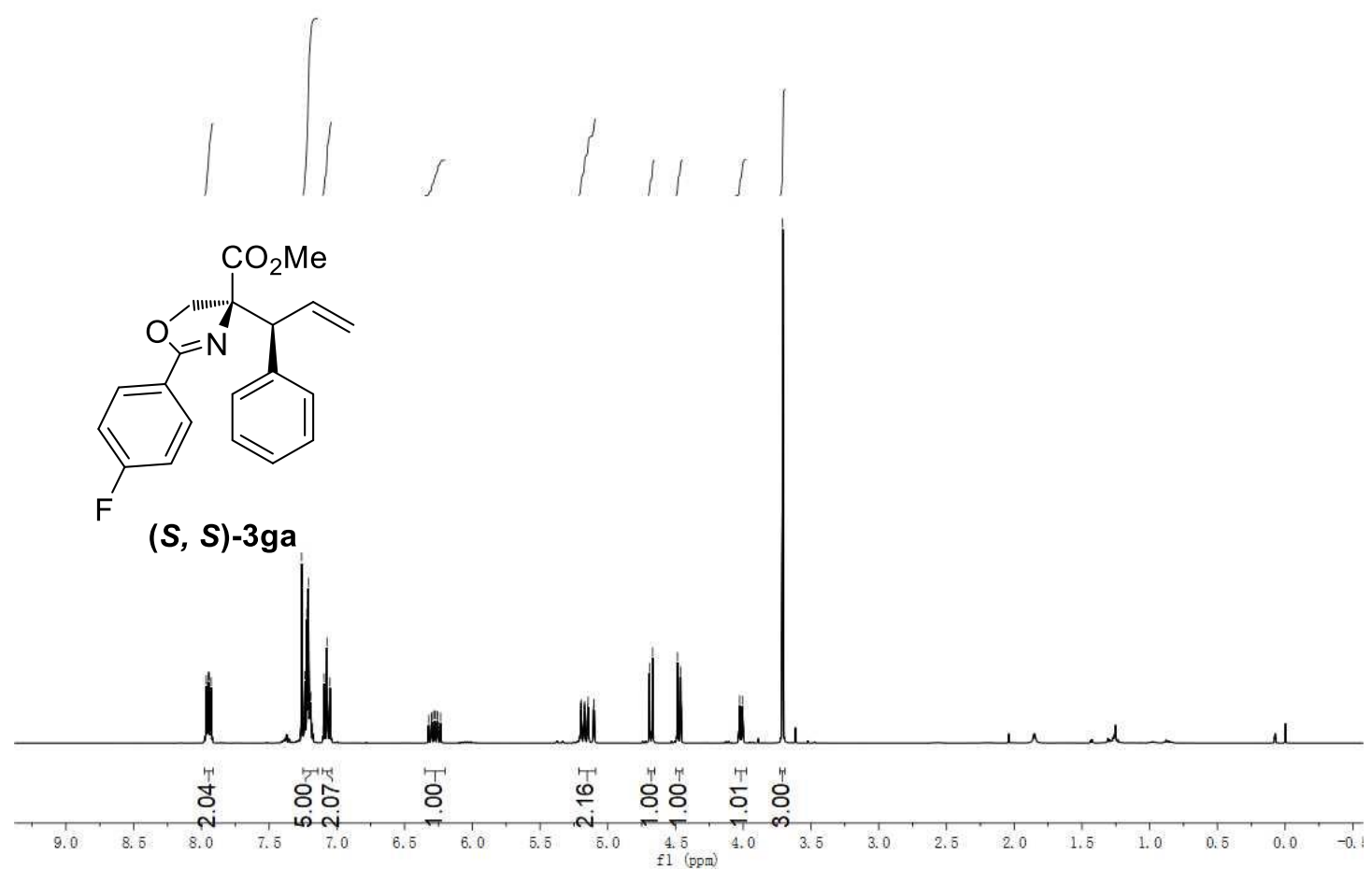

${ }^{1} \mathbf{H}$ NMR $\left(400 \mathrm{MHz}, \mathrm{CDCl}_{3}\right)$
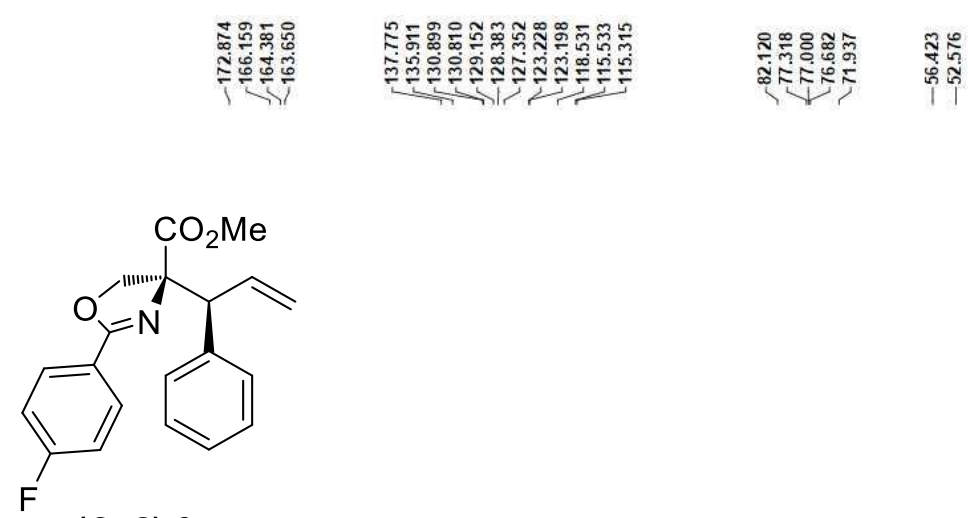

$(S, S)-3 g a$
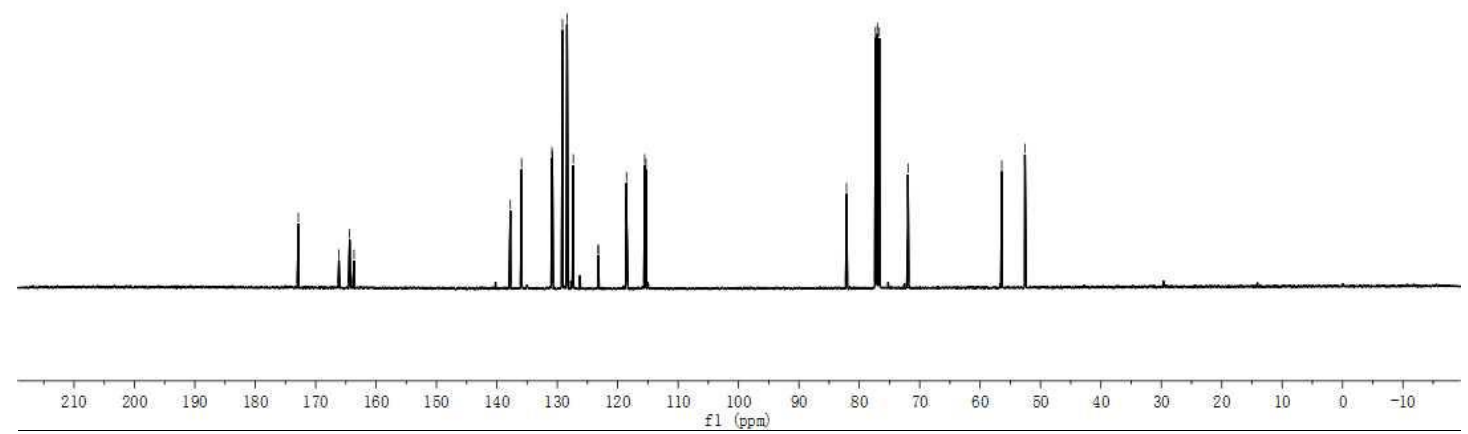

${ }^{13}$ C NMR (100 MHz, $\left.\mathrm{CDCl}_{3}\right)$ 


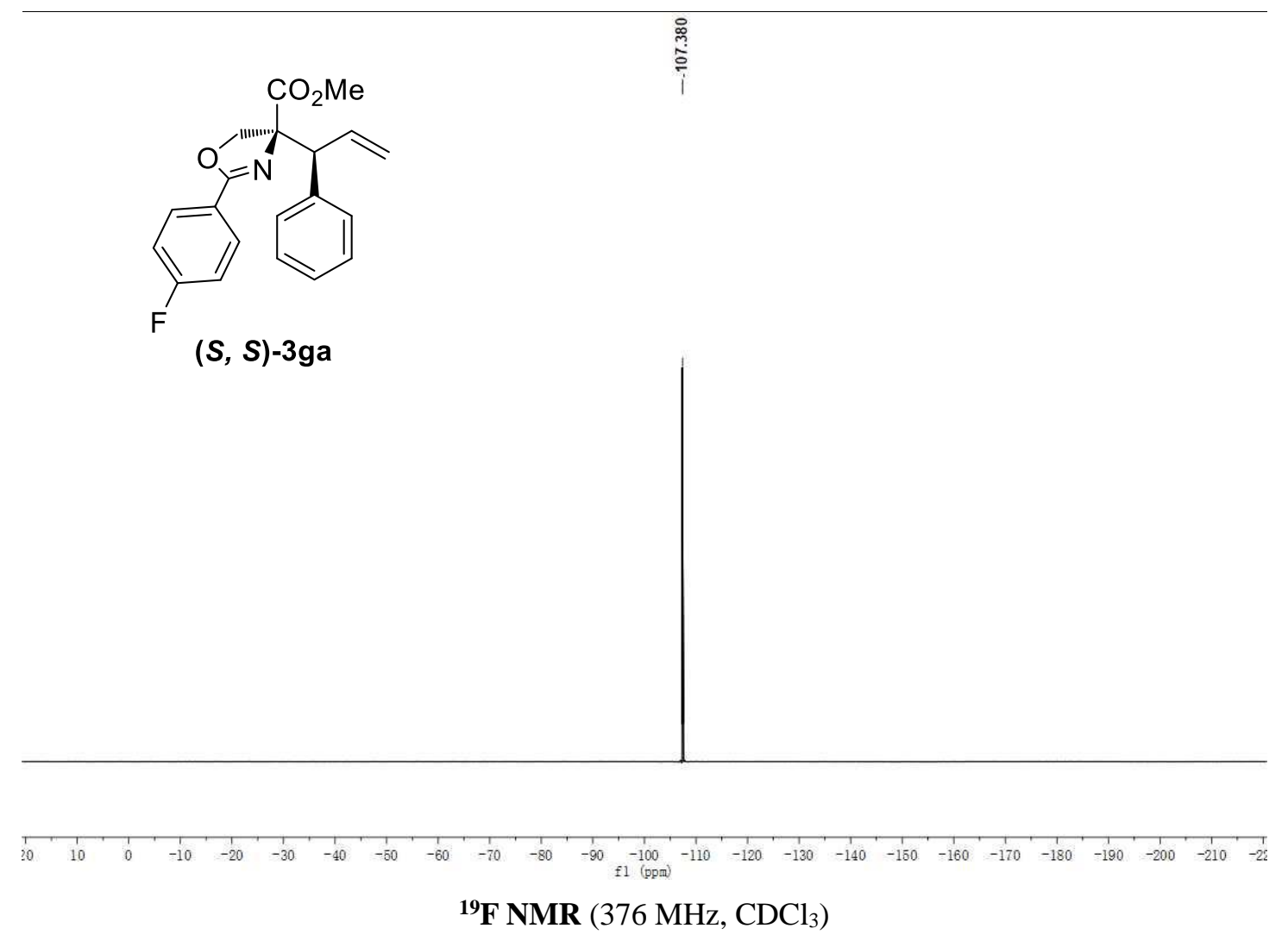




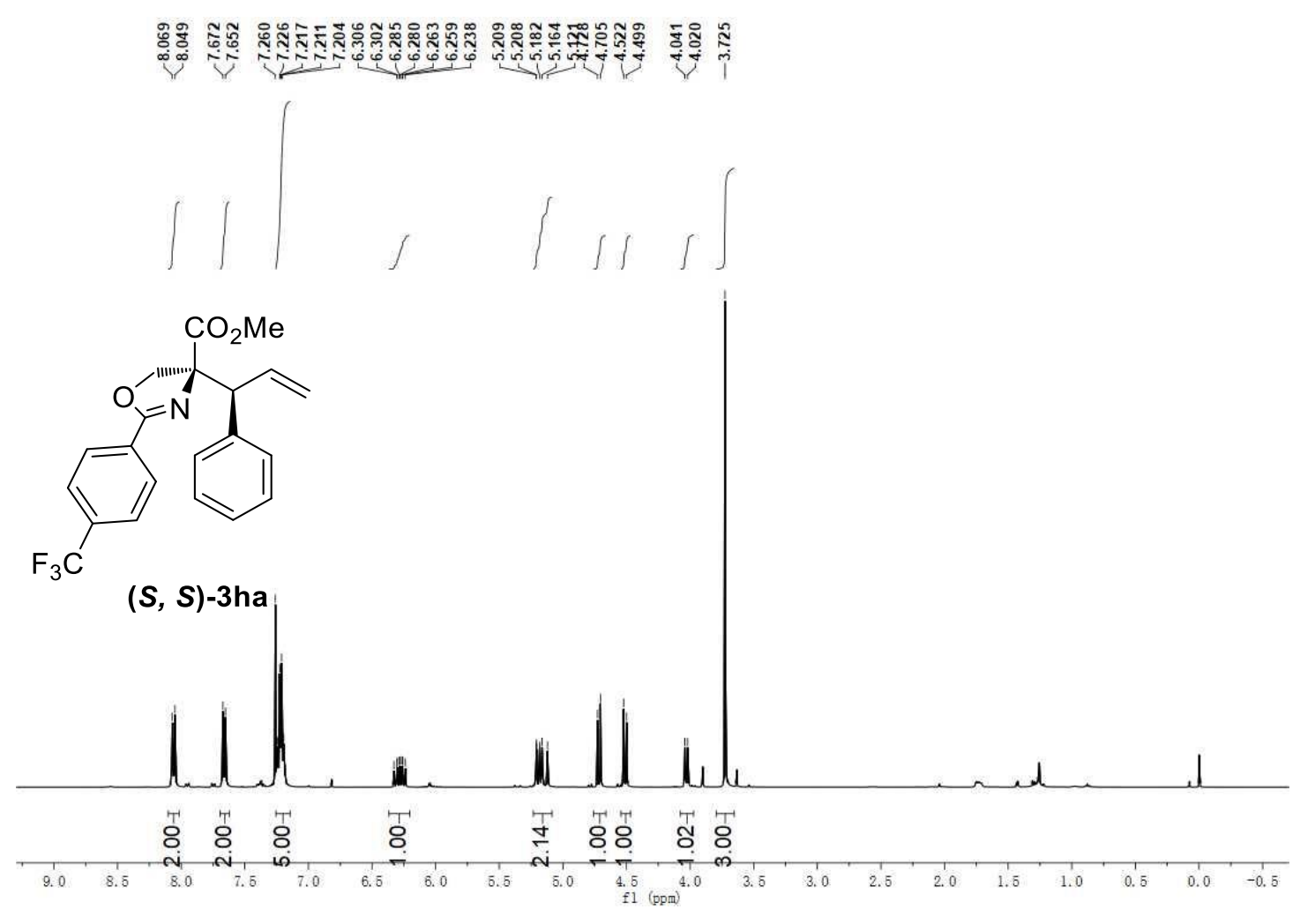

${ }^{1} \mathbf{H}$ NMR $\left(400 \mathrm{MHz}, \mathrm{CDCl}_{3}\right)$

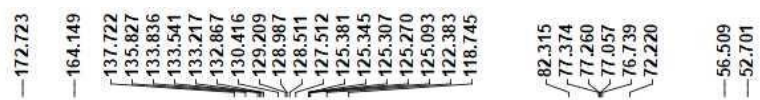

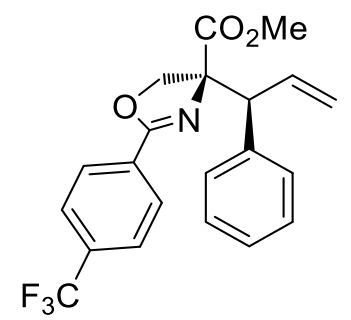

$(S, S)-3$ ha
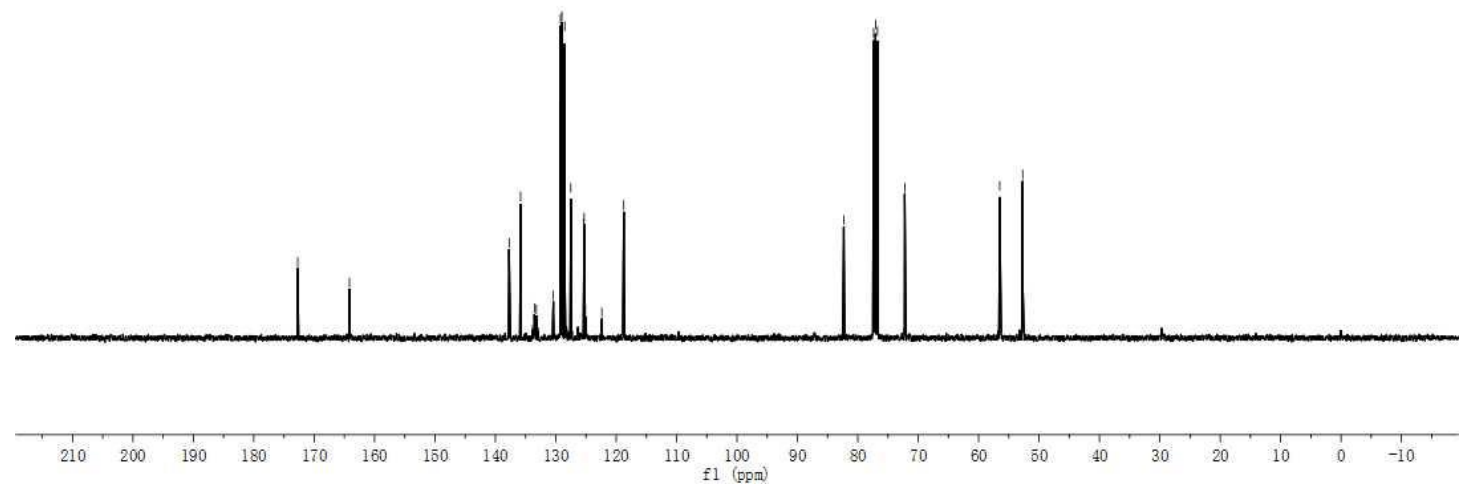

${ }^{13} \mathbf{C}$ NMR $\left(100 \mathrm{MHz}, \mathrm{CDCl}_{3}\right)$ 


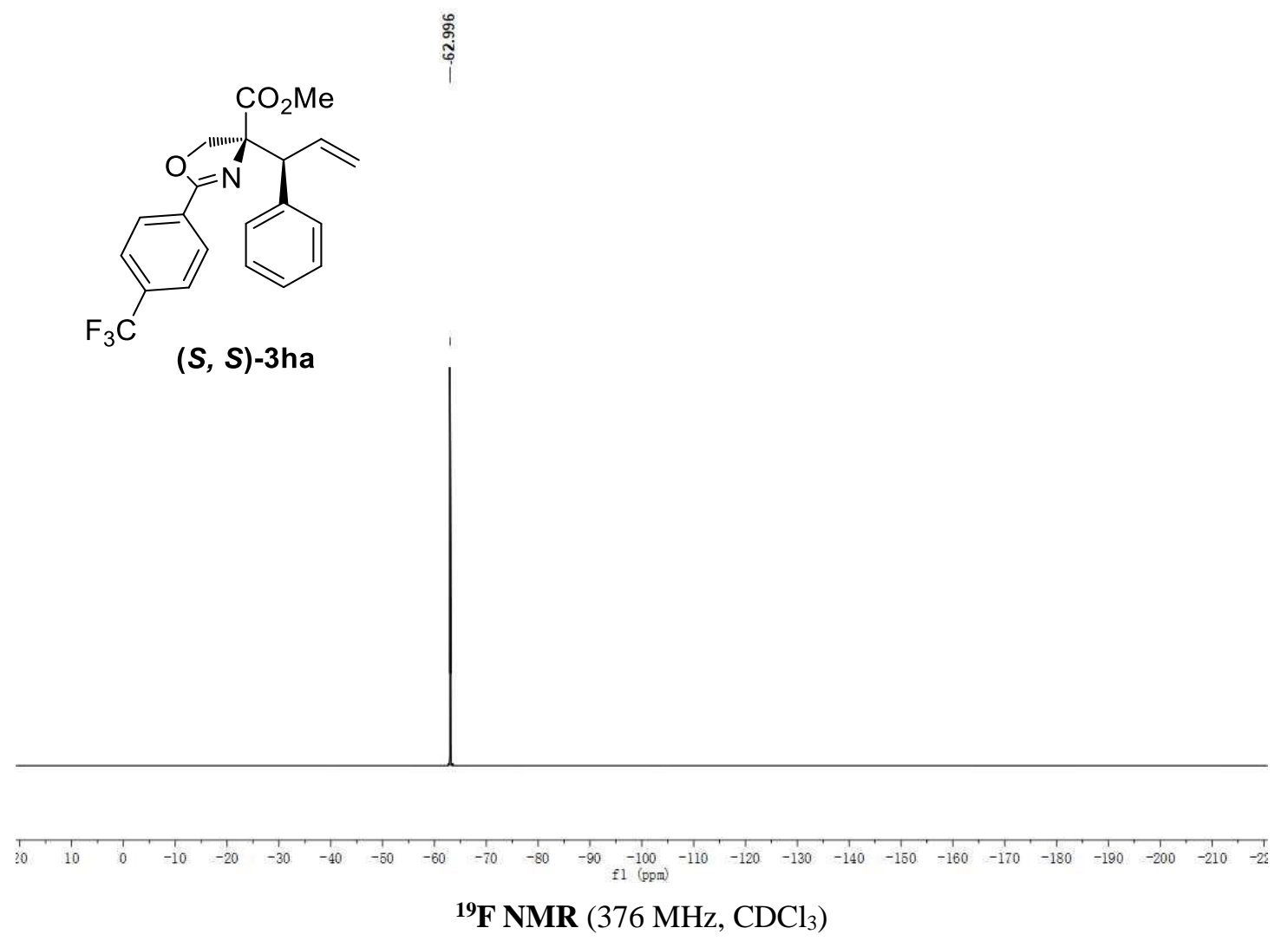




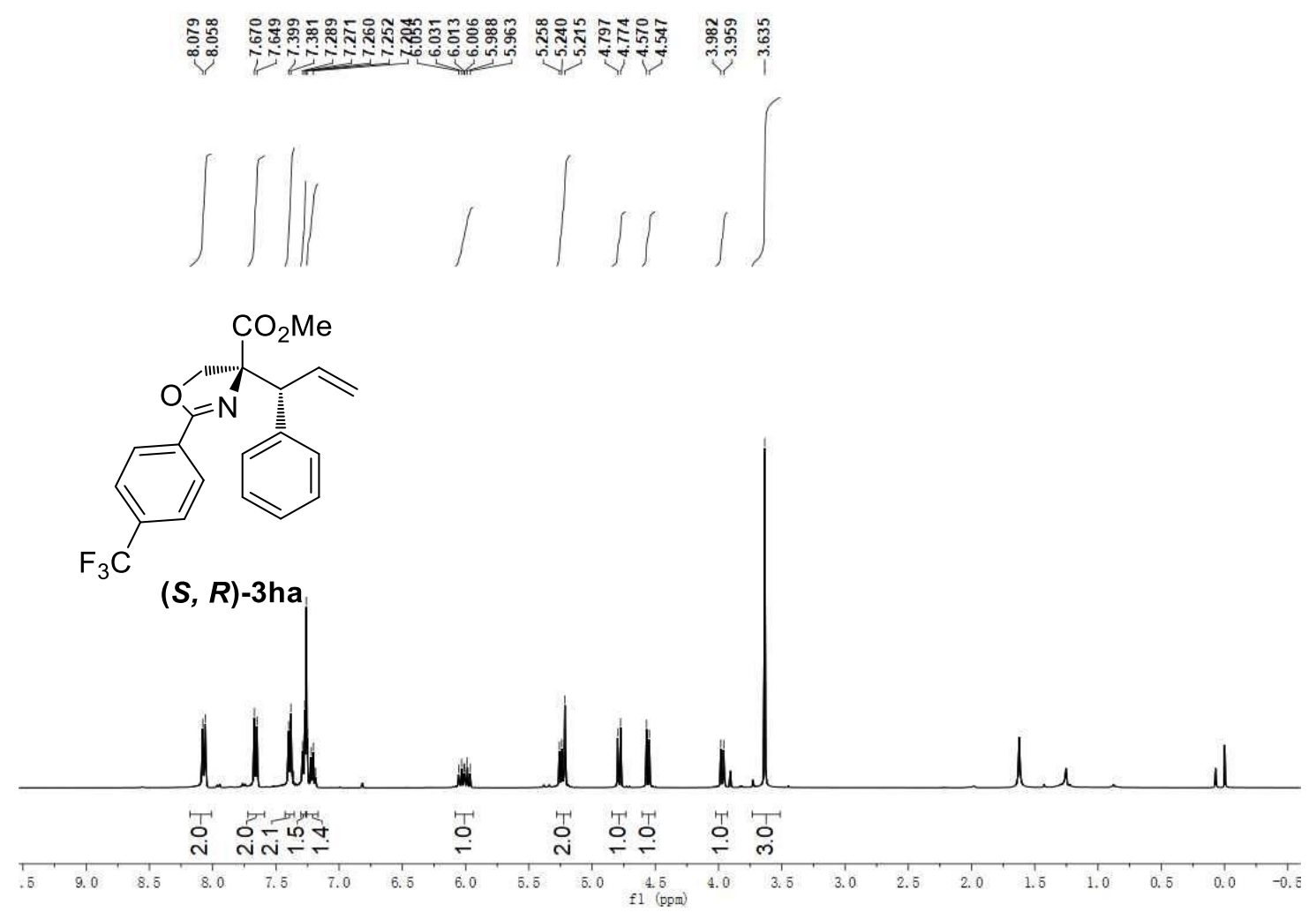

${ }^{1} \mathbf{H}$ NMR $\left(400 \mathrm{MHz}, \mathrm{CDCl}_{3}\right)$

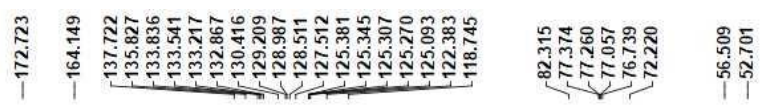

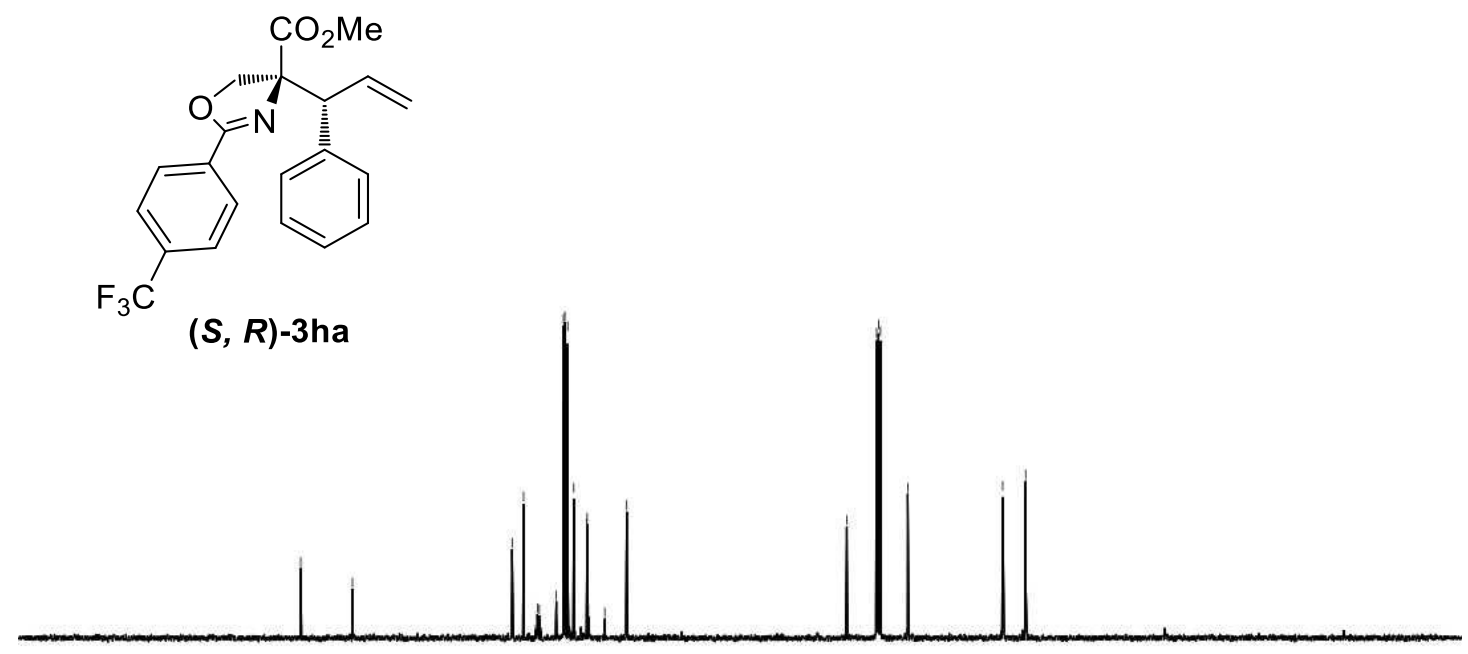

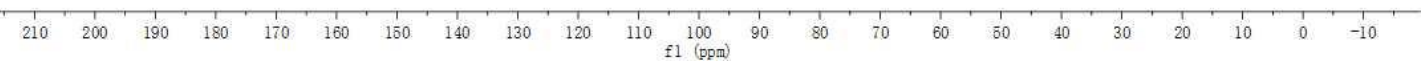

${ }^{13}$ C NMR (100 MHz, $\left.\mathrm{CDCl}_{3}\right)$ 

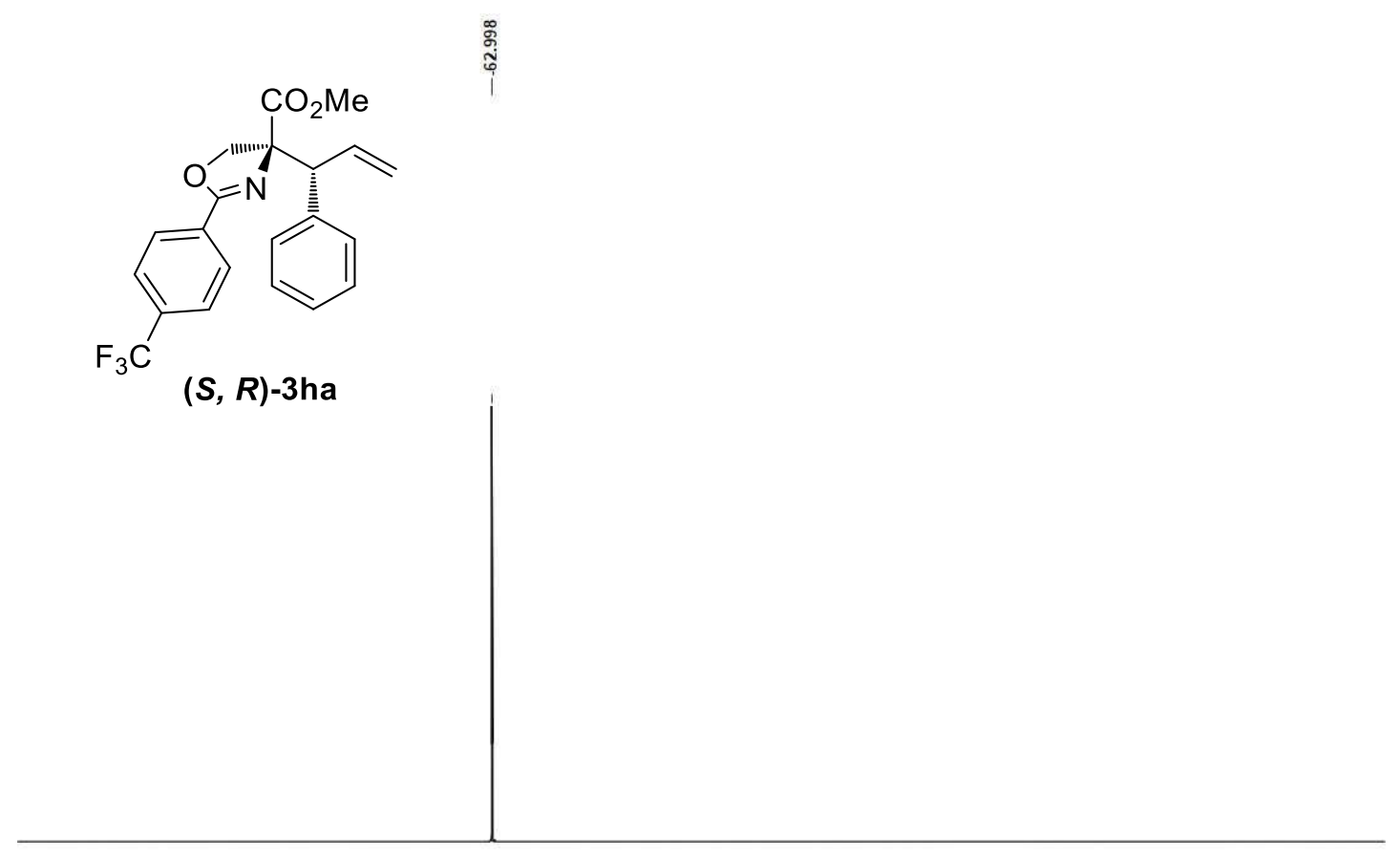

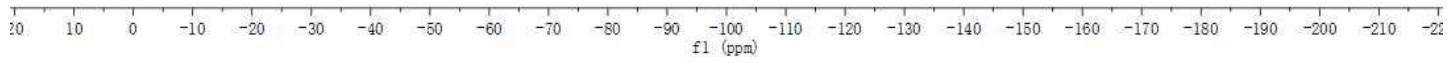

${ }^{19}$ F NMR $\left(376 \mathrm{MHz}, \mathrm{CDCl}_{3}\right)$ 


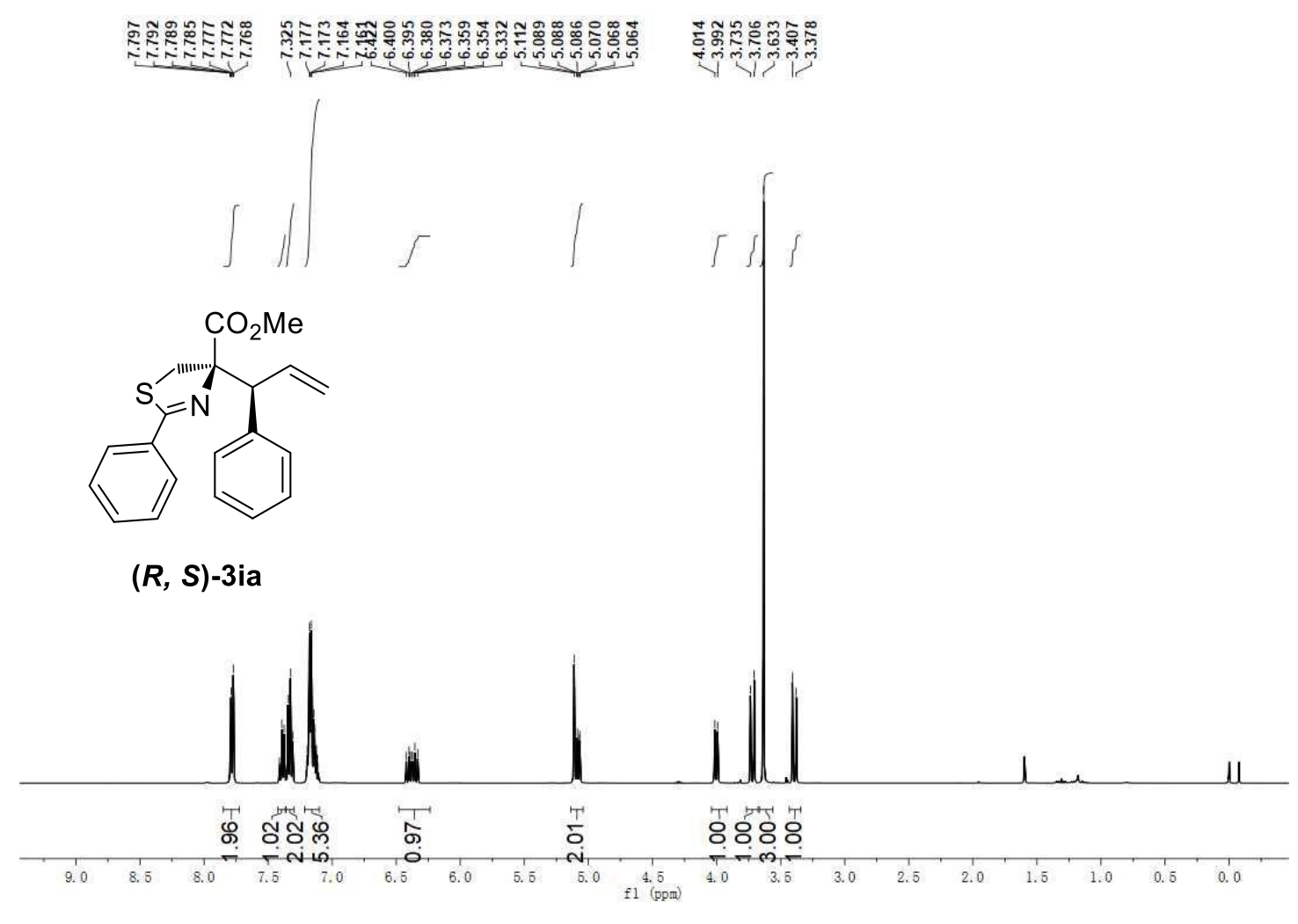

${ }^{1} \mathbf{H}$ NMR $\left(400 \mathrm{MHz}, \mathrm{CDCl}_{3}\right)$

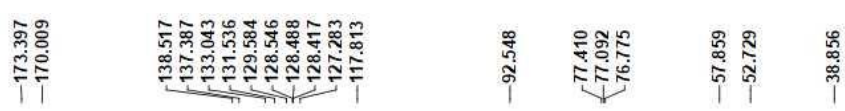
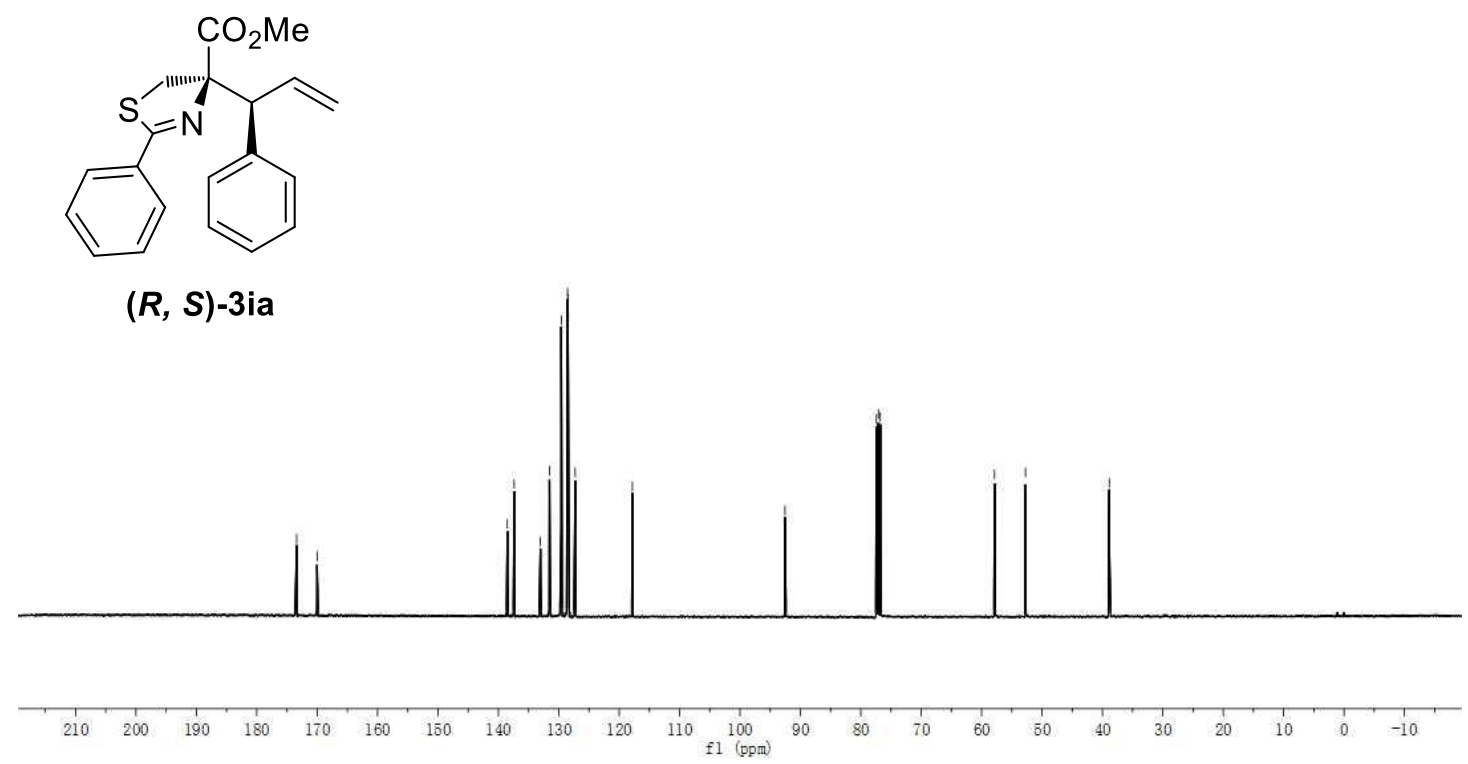

${ }^{13}$ C NMR (100 MHz, $\left.\mathrm{CDCl}_{3}\right)$ 

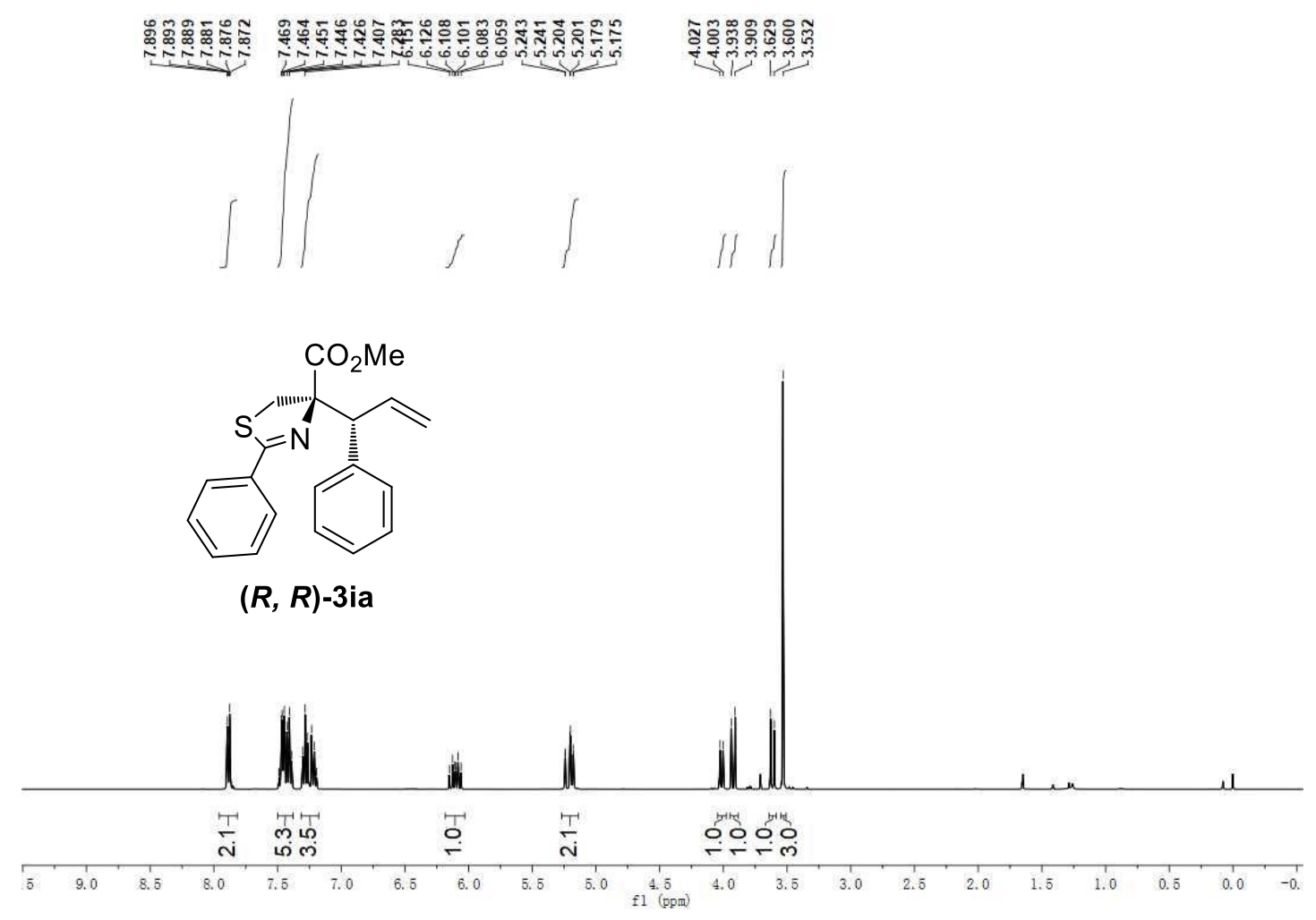

${ }^{1} \mathbf{H}$ NMR $\left(400 \mathrm{MHz}, \mathrm{CDCl}_{3}\right)$

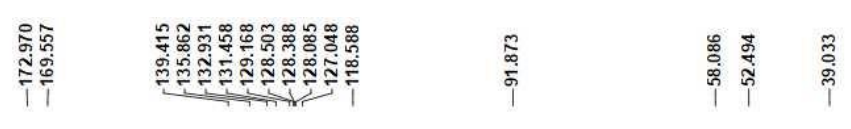

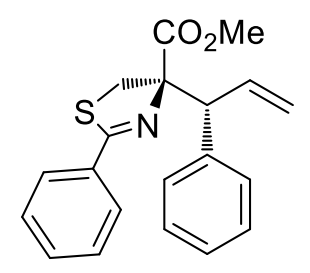

$(R, R)$-3ia
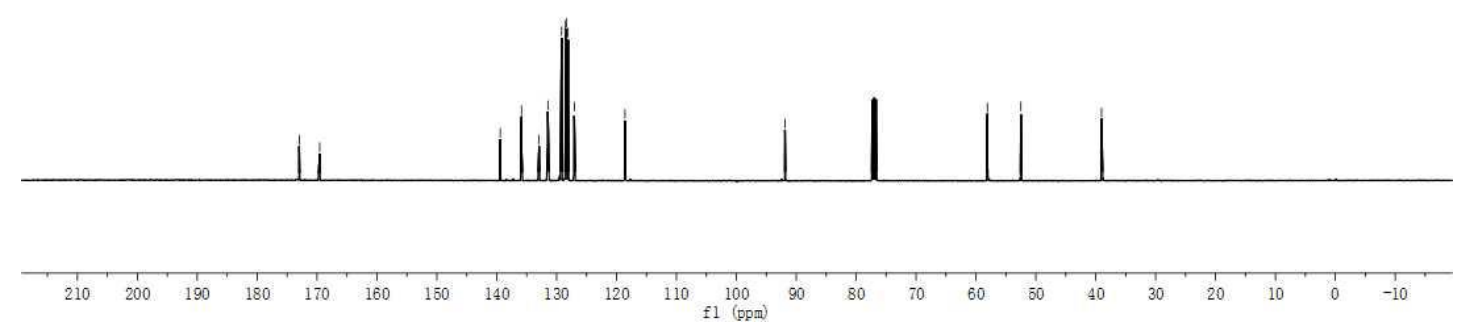

${ }^{13}$ C NMR (100 MHz, $\left.\mathrm{CDCl}_{3}\right)$ 


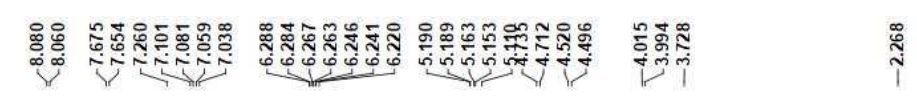
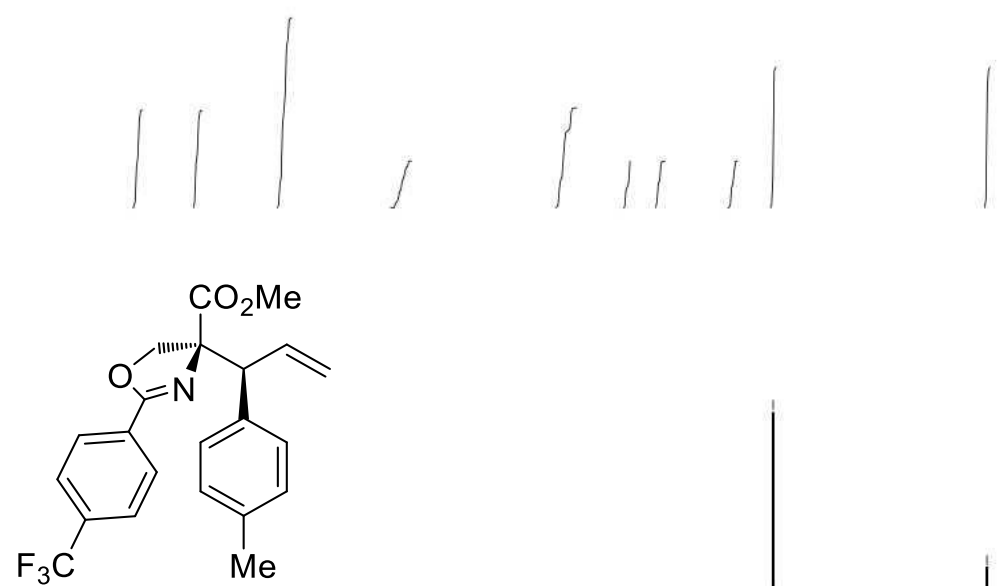

$(S, S)-3 h b$

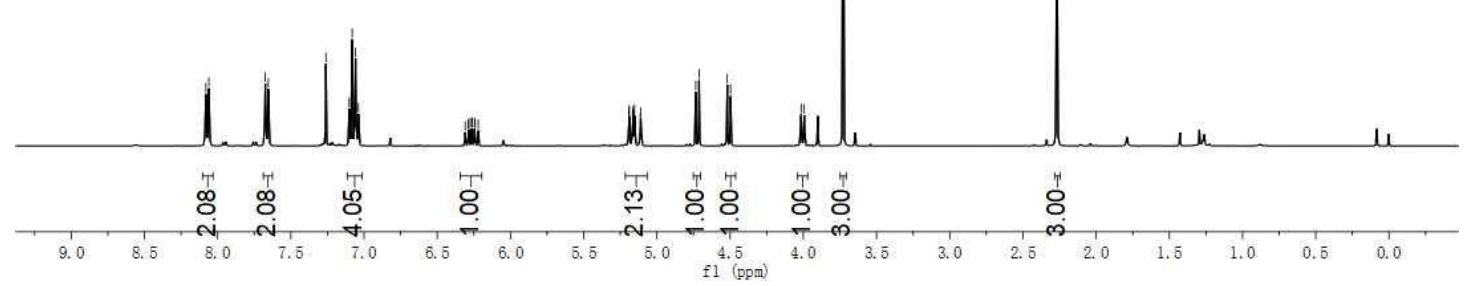

${ }^{1} \mathbf{H}$ NMR $\left(400 \mathrm{MHz}, \mathrm{CDCl}_{3}\right)$
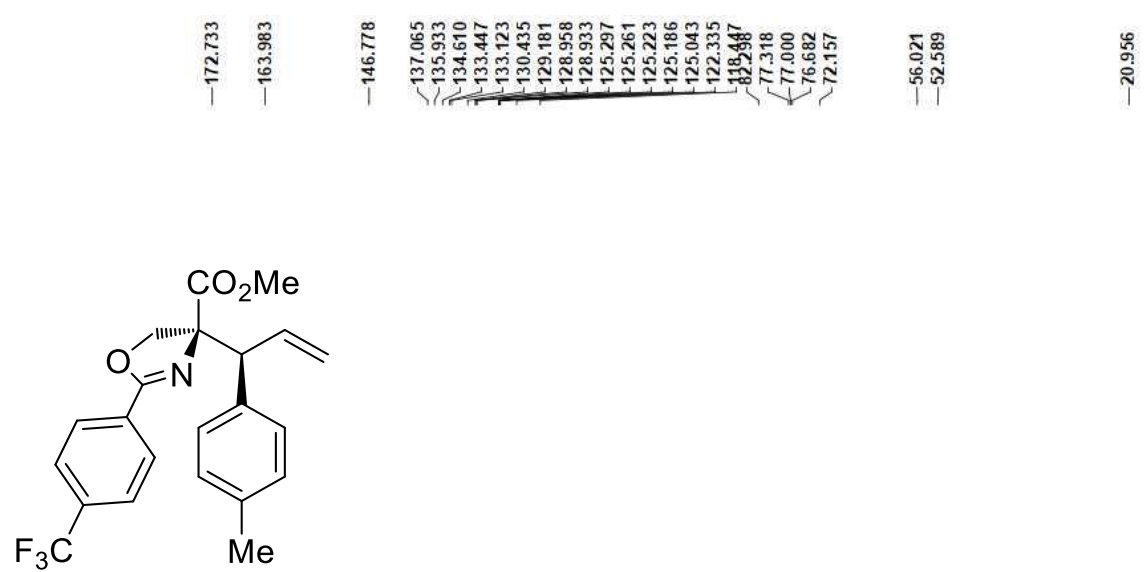

$(S, S)-3 h b$

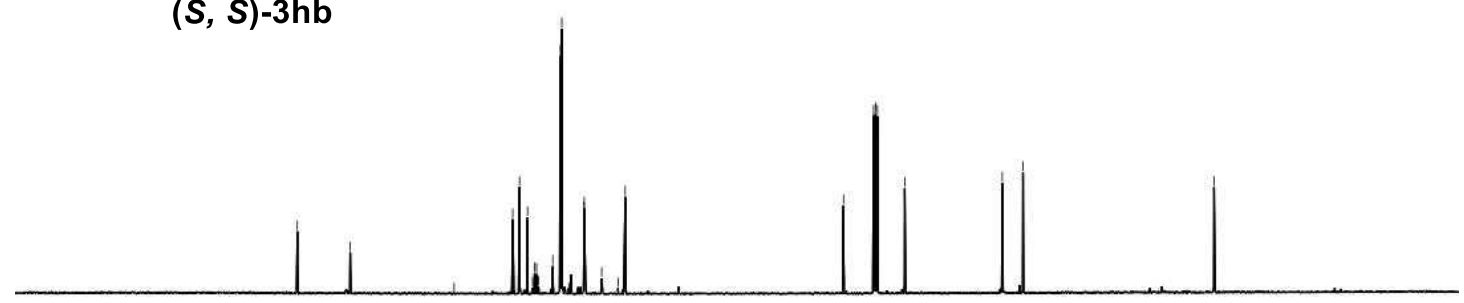

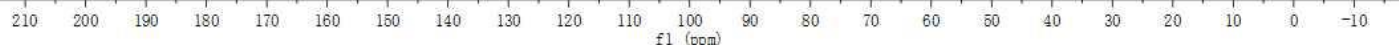

${ }^{13} \mathbf{C}$ NMR $\left(100 \mathrm{MHz}, \mathrm{CDCl}_{3}\right)$ 
ֻ

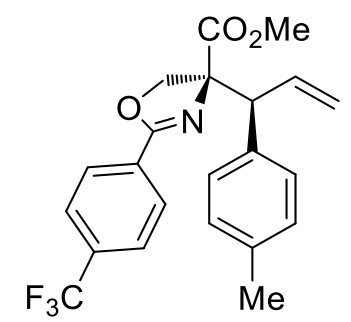

$(S, S)-3 h b$

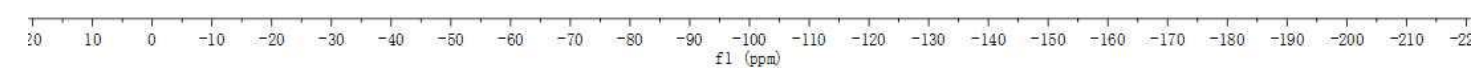

${ }^{19} \mathbf{F}$ NMR $\left(376 \mathrm{MHz}, \mathrm{CDCl}_{3}\right)$ 


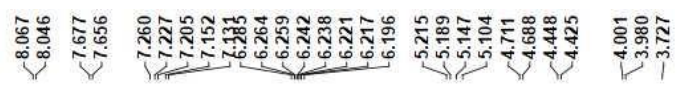
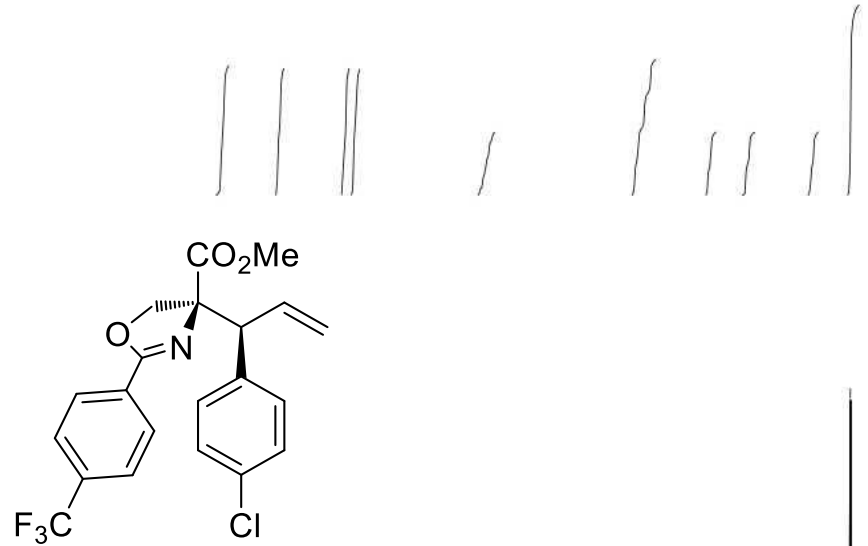

(S, S)-3hc

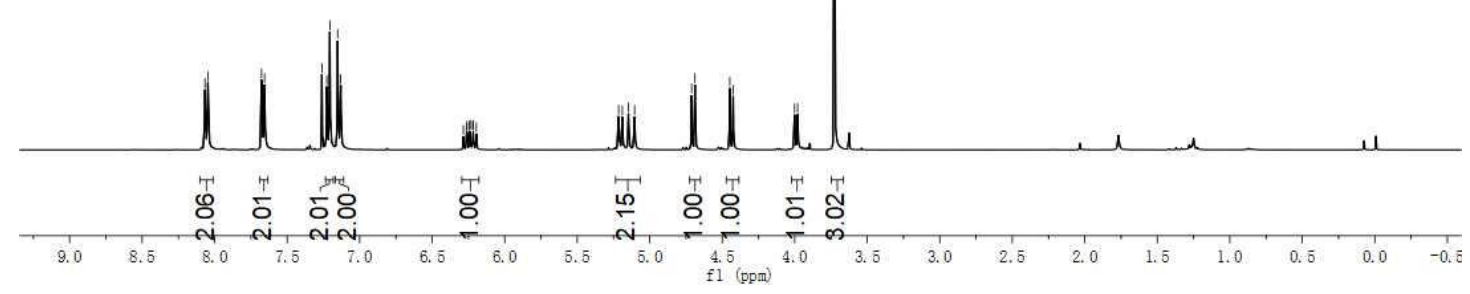

${ }^{1} \mathbf{H}$ NMR $\left(400 \mathrm{MHz}, \mathrm{CDCl}_{3}\right)$
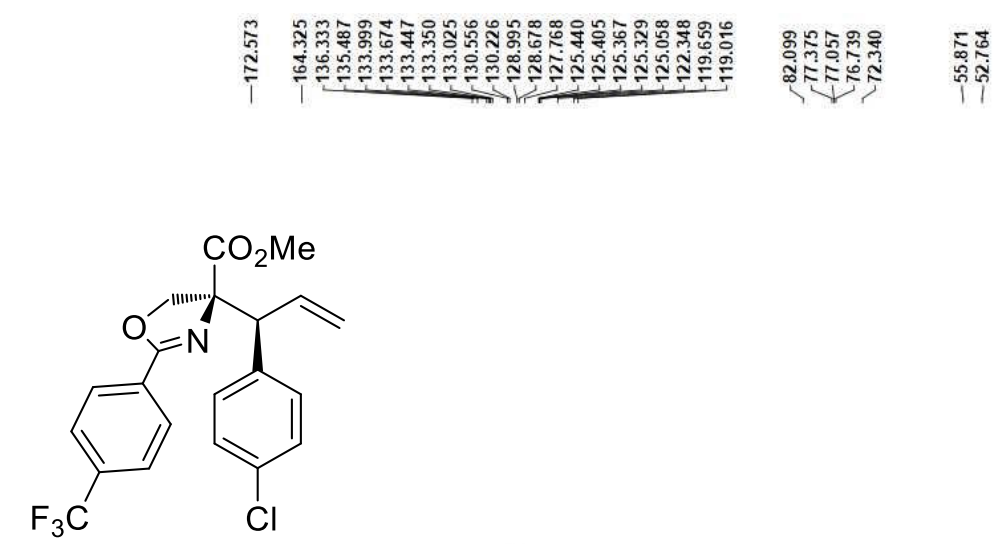

(S, S)-3hc
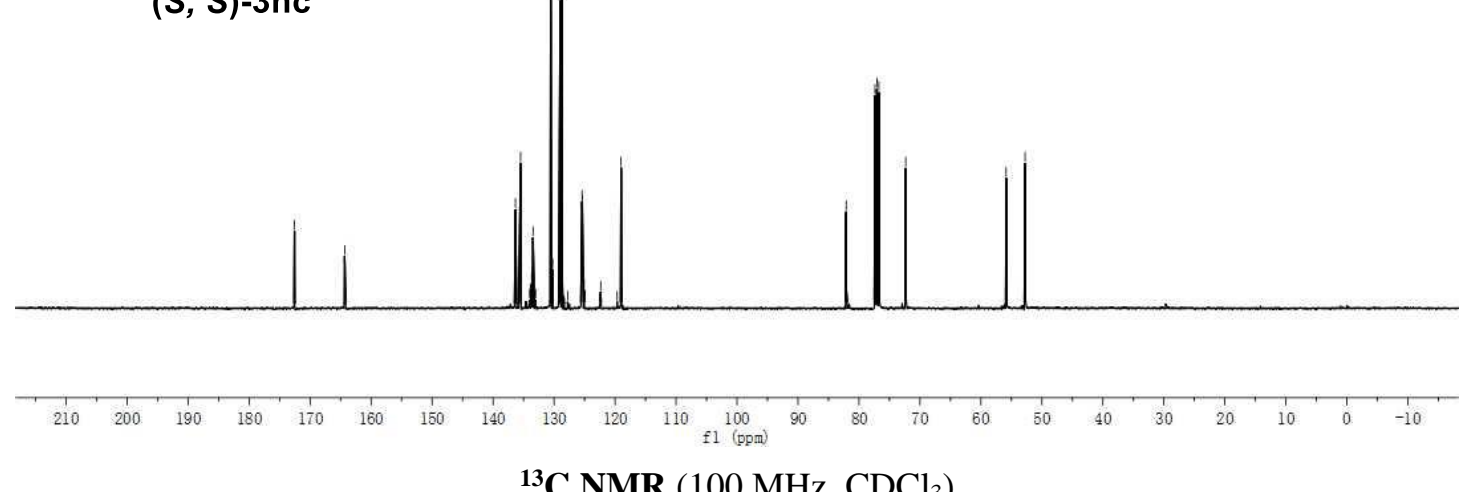

${ }^{13} \mathbf{C}$ NMR $\left(100 \mathrm{MHz}, \mathrm{CDCl}_{3}\right)$ 


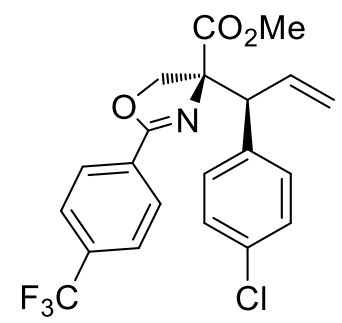

$(S, S)-3 \mathrm{hc}$

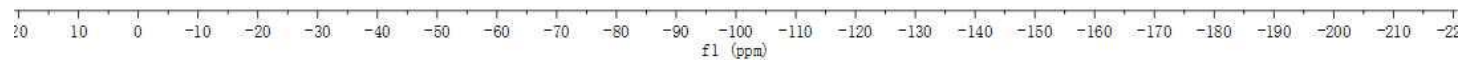

${ }^{19} \mathbf{F}$ NMR $\left(376 \mathrm{MHz}, \mathrm{CDCl}_{3}\right)$ 


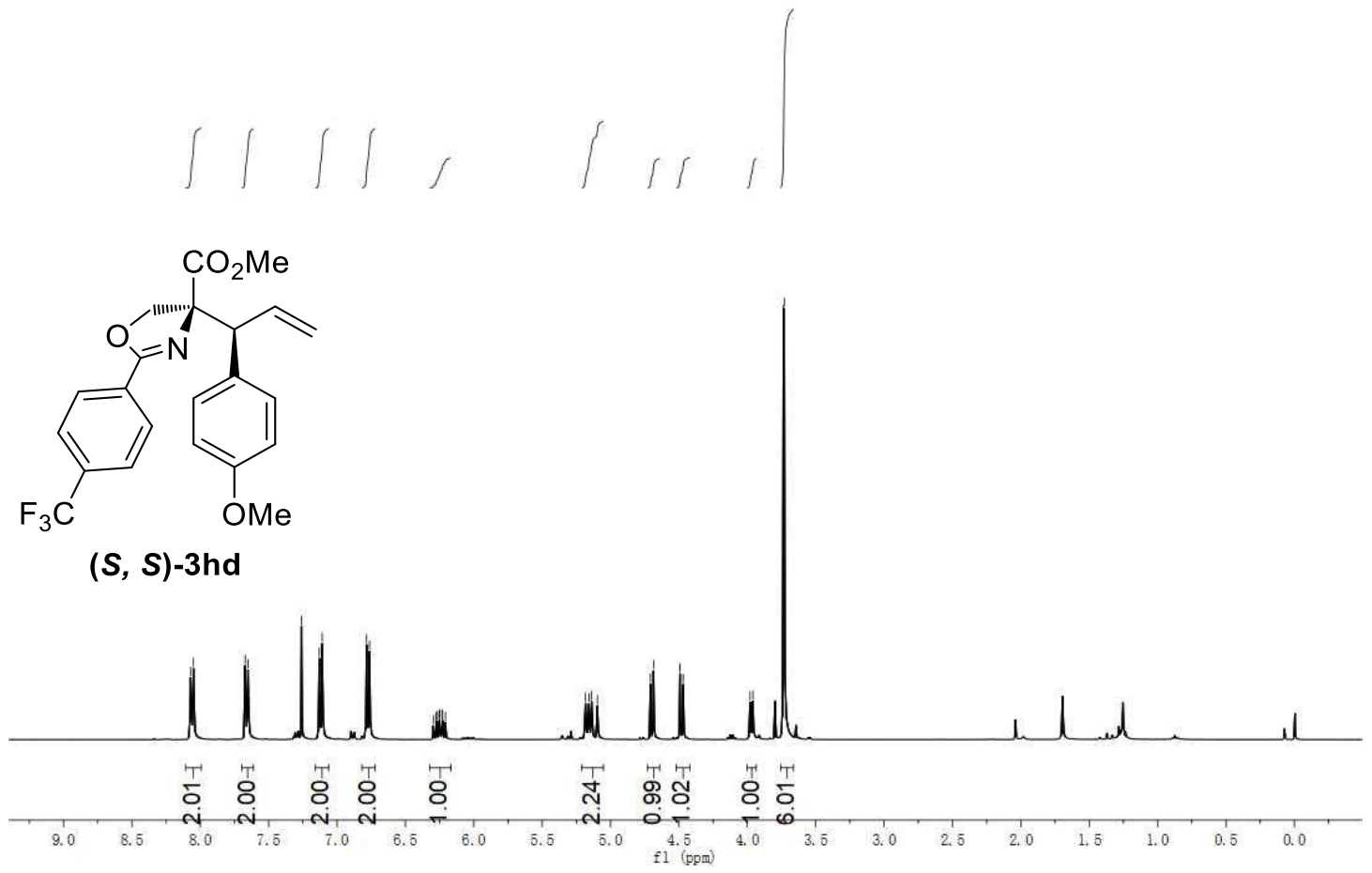

${ }^{1} \mathbf{H}$ NMR $\left(400 \mathrm{MHz}, \mathrm{CDCl}_{3}\right)$

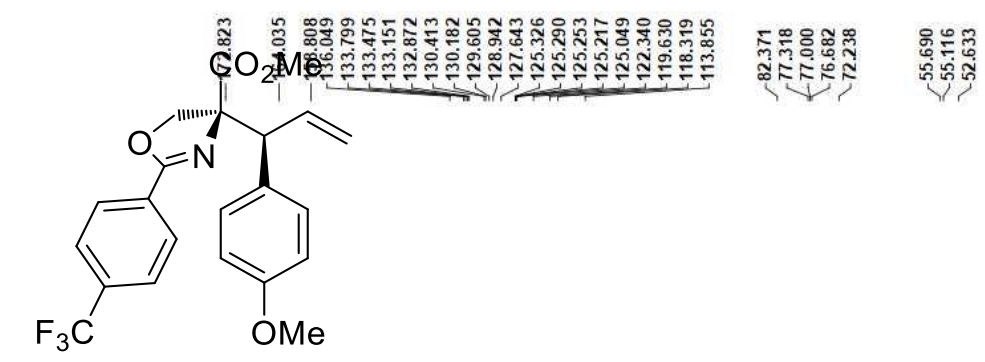

$(S, S)-3 h d$
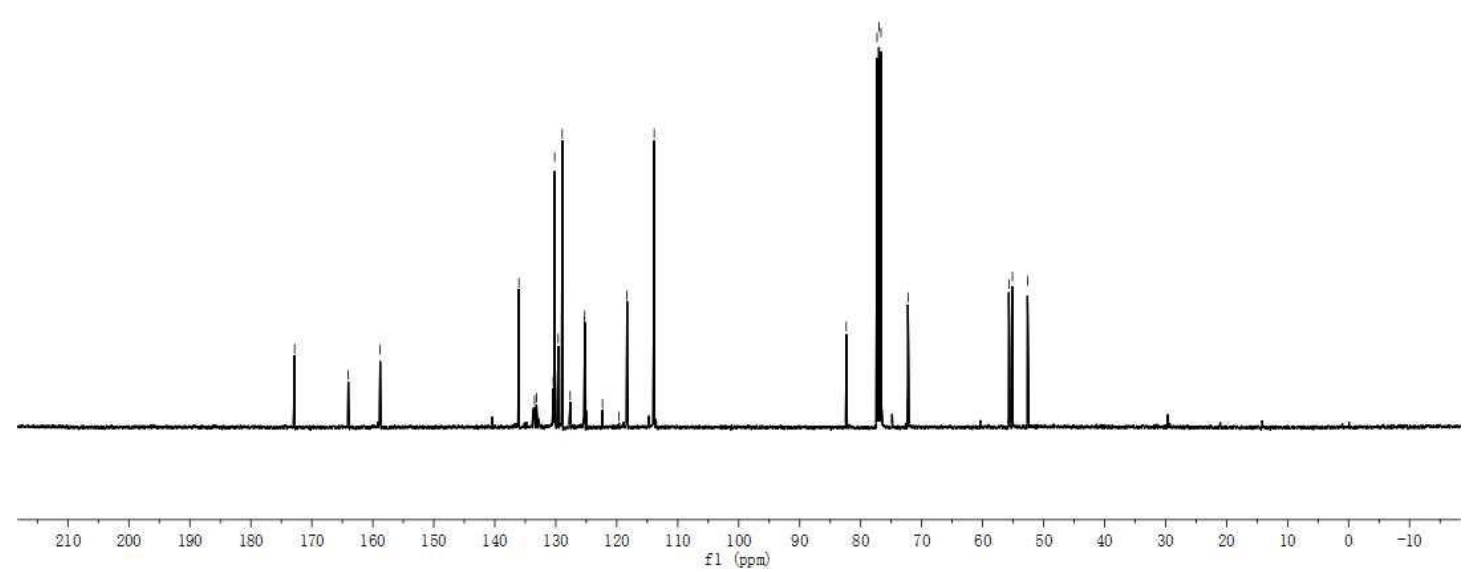

${ }^{13} \mathbf{C}$ NMR $\left(100 \mathrm{MHz}, \mathrm{CDCl}_{3}\right)$ 


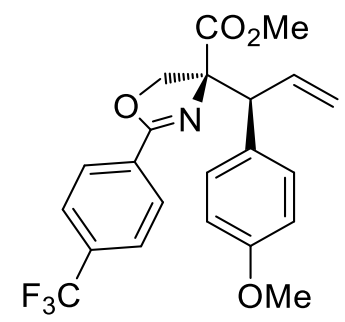

(S, S)-3hd

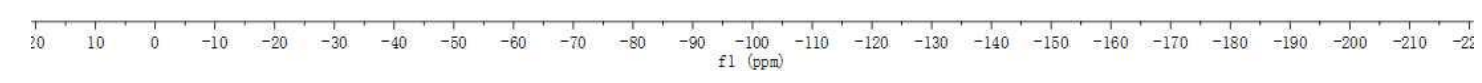

${ }^{19}$ F NMR (376 MHz, $\left.\mathrm{CDCl}_{3}\right)$ 


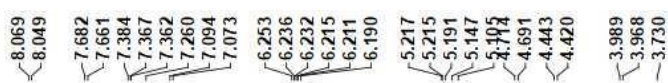

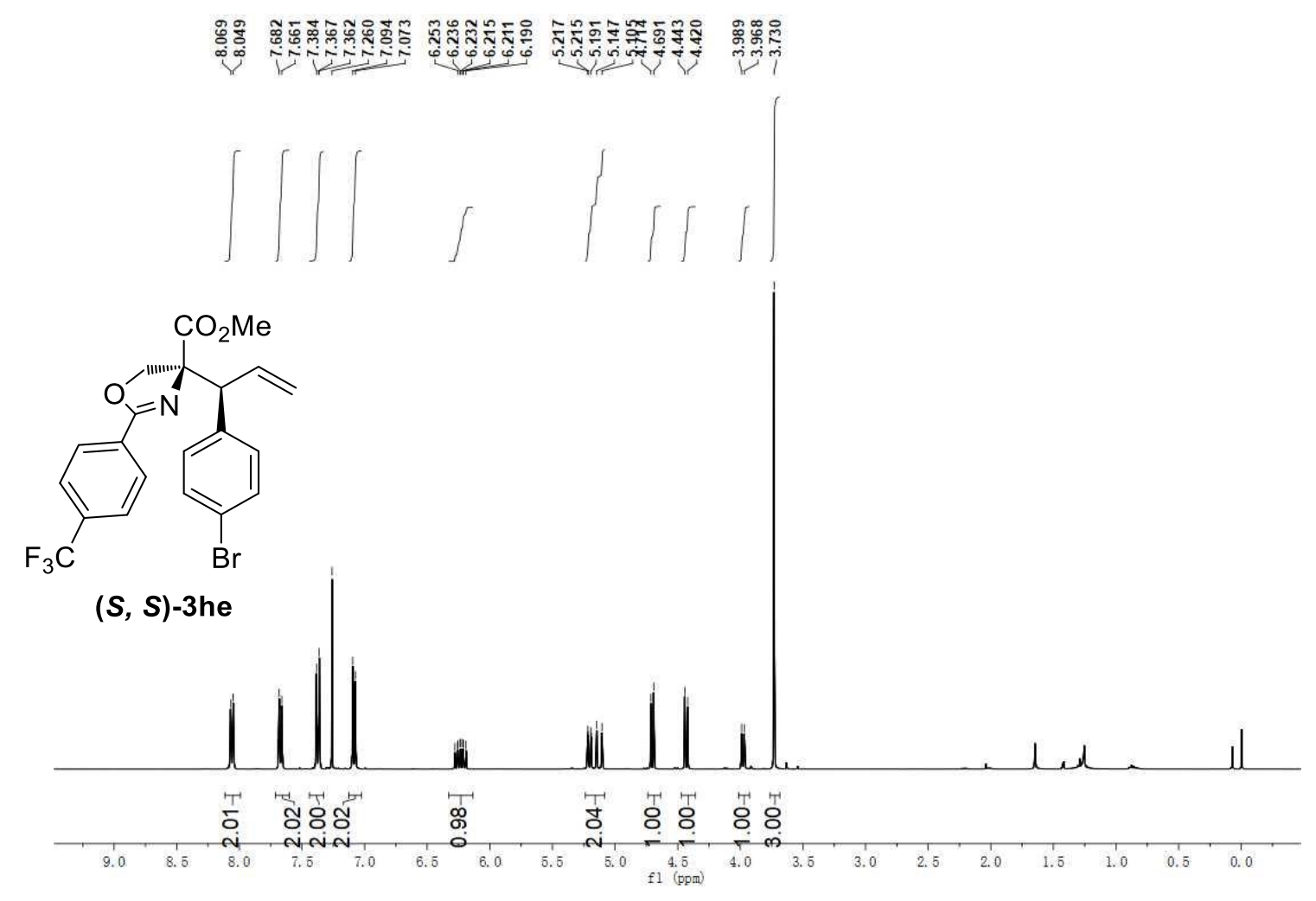

${ }^{1} \mathbf{H}$ NMR $\left(400 \mathrm{MHz}, \mathrm{CDCl}_{3}\right)$
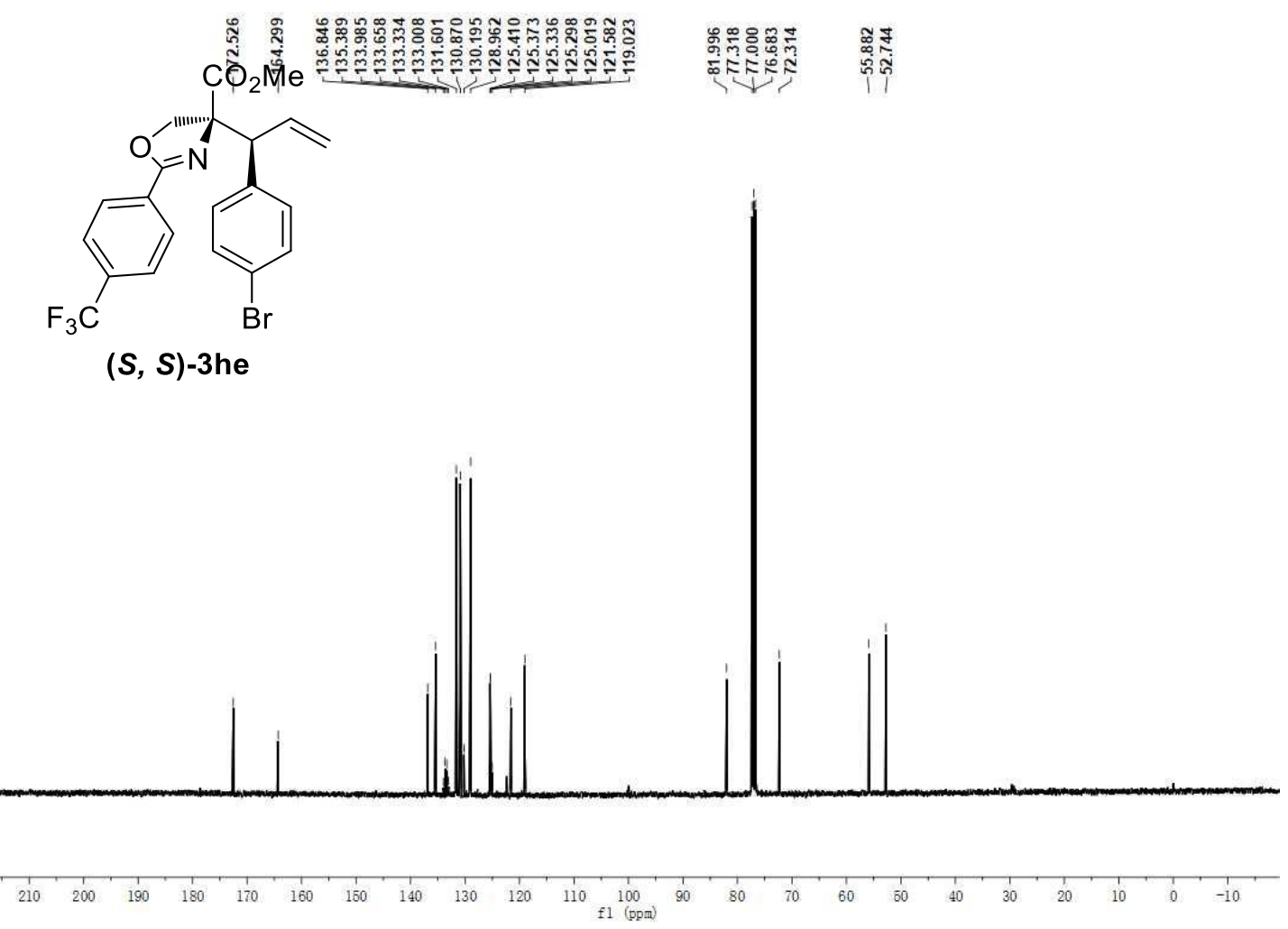

${ }^{13}$ C NMR (100 MHz, $\left.\mathrm{CDCl}_{3}\right)$ 
$\stackrel{0}{\circ}$
ஸீ
$i$<smiles>C=CC(c1ccc(Br)cc1)C(C)(C)N1C=C(c2ccc(C(F)(F)F)cc2)OC1</smiles>

$(S, S)$-3he

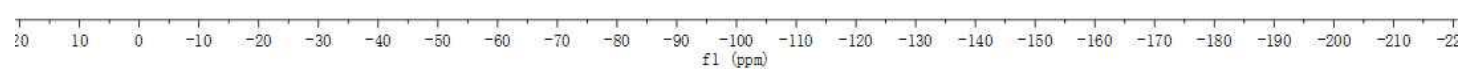

${ }^{19} \mathbf{F}$ NMR $\left(376 \mathrm{MHz}, \mathrm{CDCl}_{3}\right)$ 

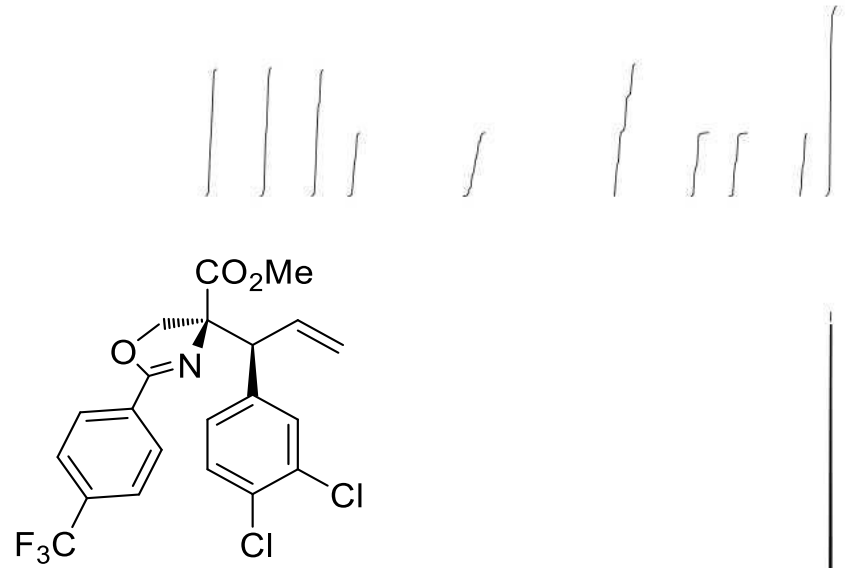

(S, S)-3hf

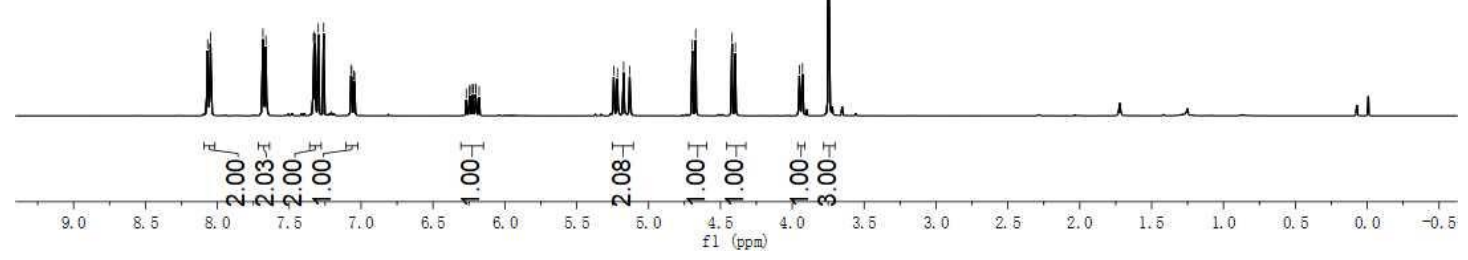

${ }^{1} \mathbf{H}$ NMR $\left(400 \mathrm{MHz}, \mathrm{CDCl}_{3}\right)$
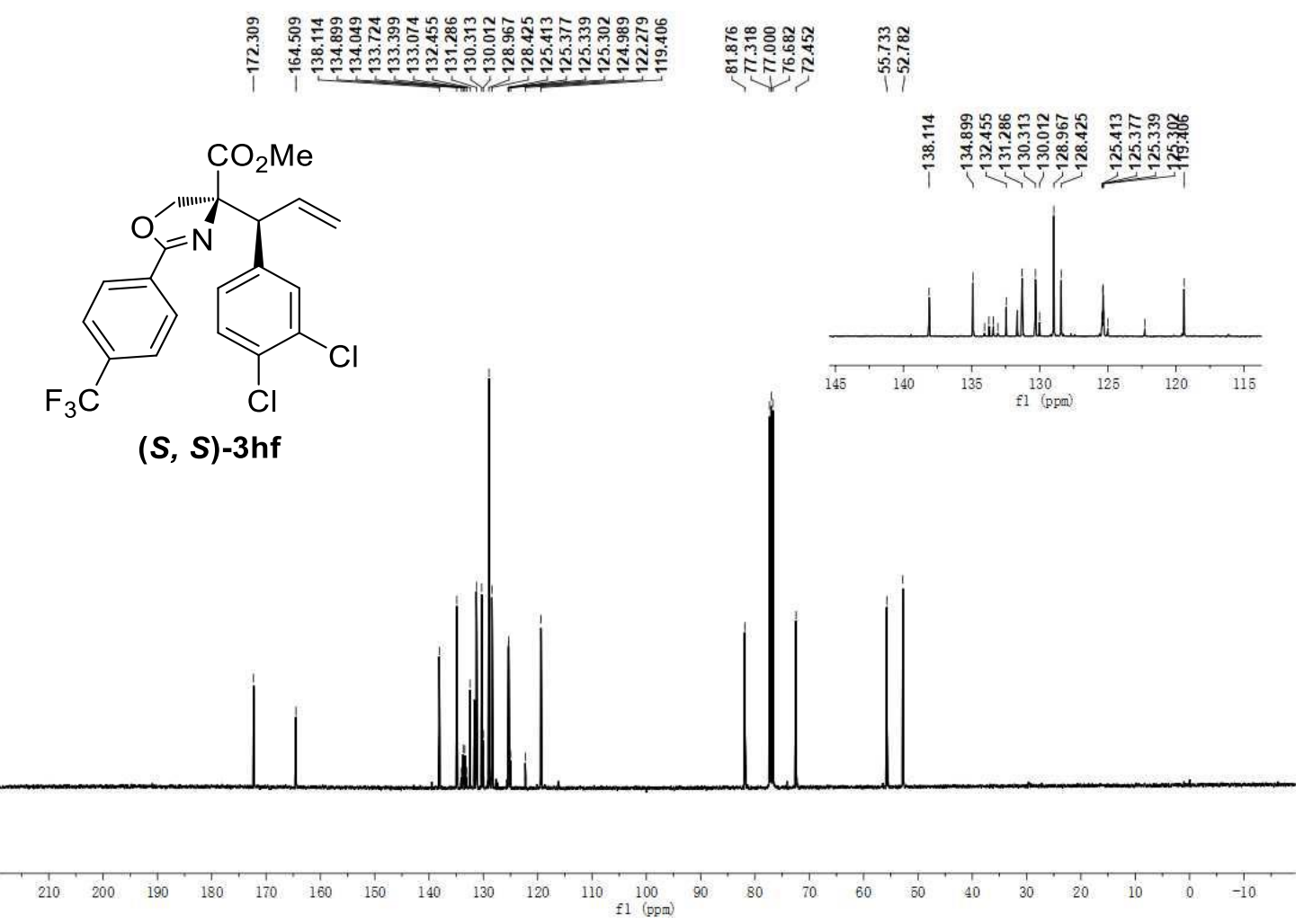

${ }^{13} \mathbf{C}$ NMR (100 MHz, $\left.\mathrm{CDCl}_{3}\right)$ 


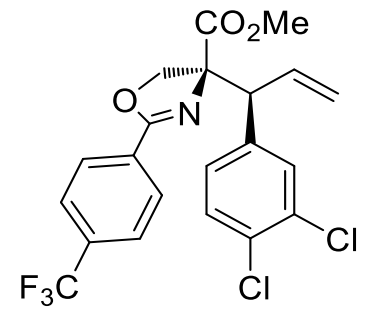

$(S, S)-3 h f$

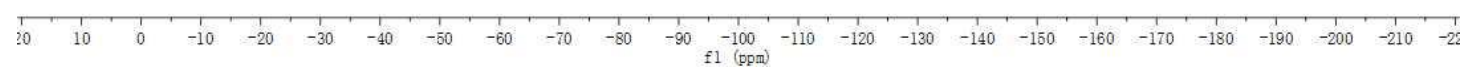

${ }^{19} \mathbf{F}$ NMR $\left(376 \mathrm{MHz}, \mathrm{CDCl}_{3}\right)$ 


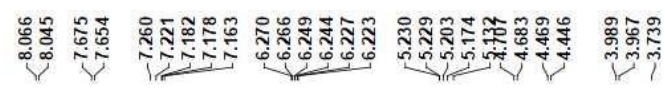
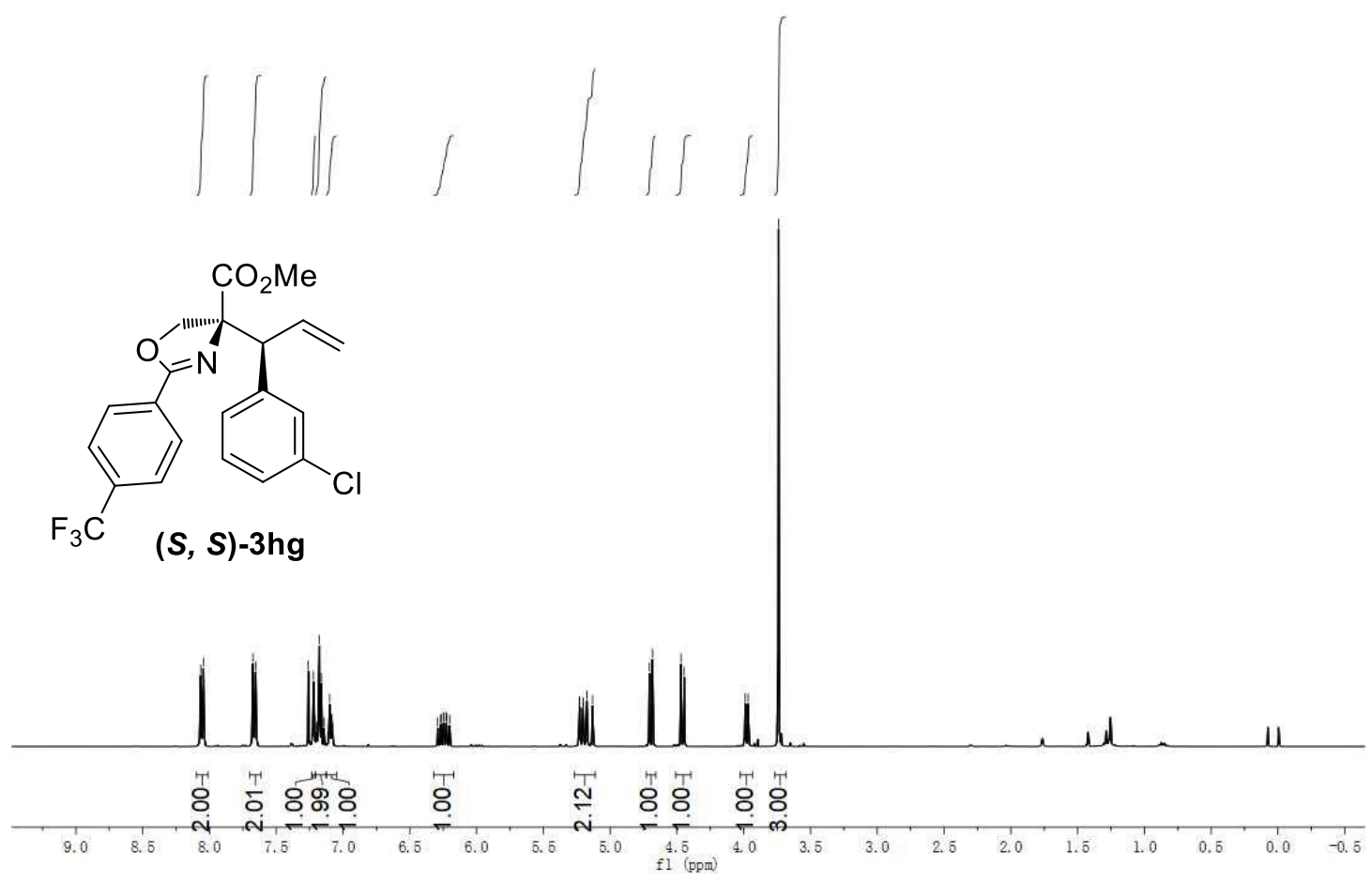

${ }^{1} \mathbf{H}$ NMR $\left(400 \mathrm{MHz}, \mathrm{CDCl}_{3}\right)$
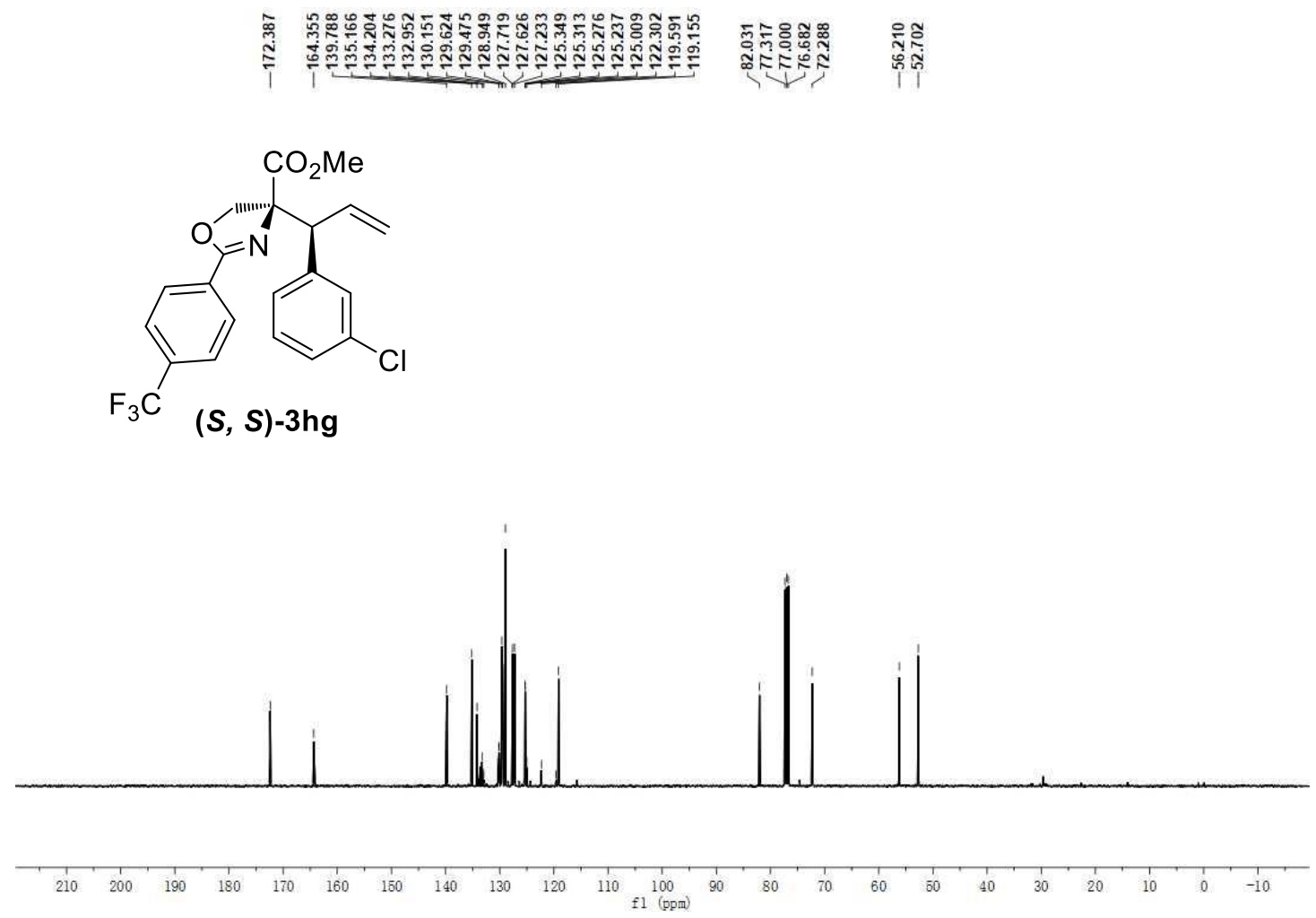

${ }^{13} \mathbf{C}$ NMR (100 MHz, $\left.\mathrm{CDCl}_{3}\right)$ 


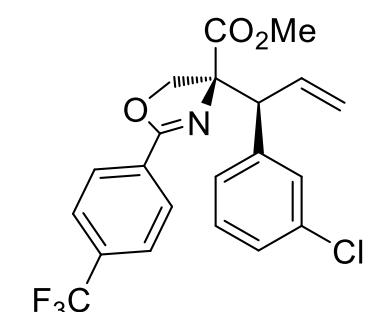

(S, S)-3hg

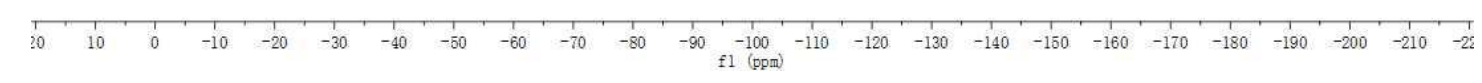

${ }^{19}$ F NMR (376 MHz, $\mathrm{CDCl}_{3}$ ) 

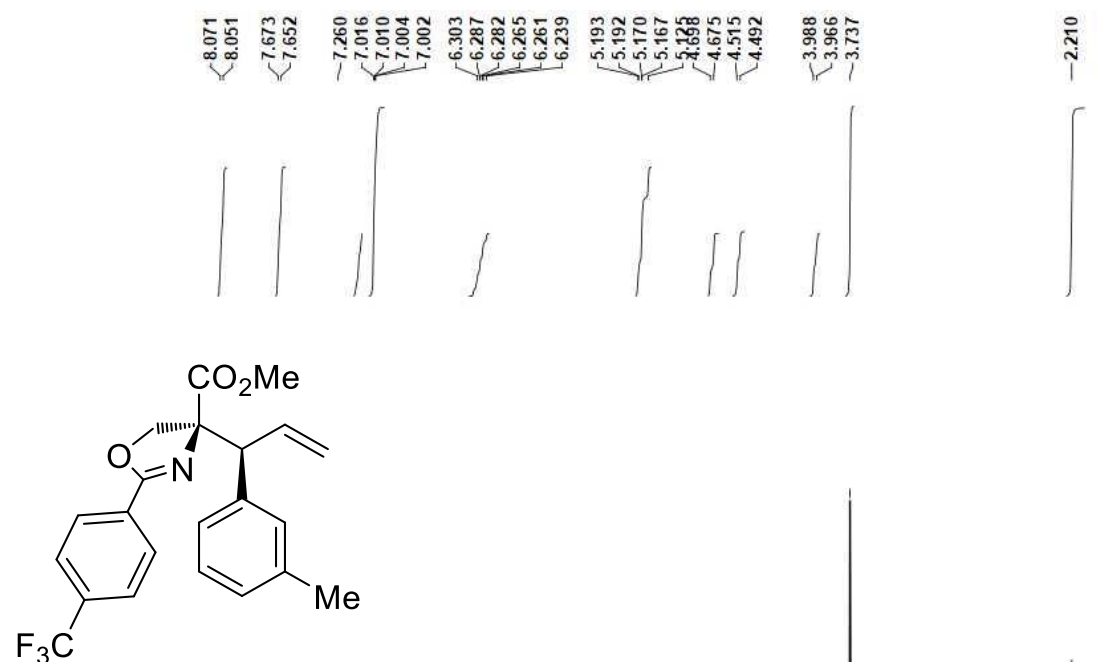

$(S, S)-3 \mathrm{hh}$

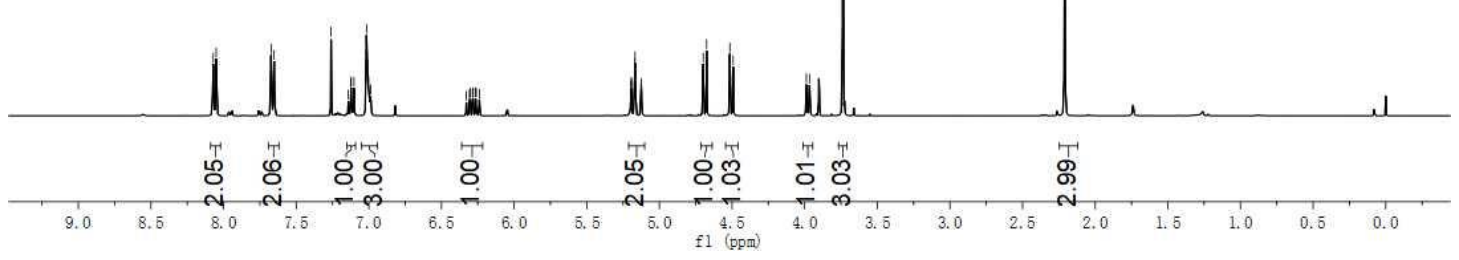

${ }^{1} \mathbf{H}$ NMR $\left(400 \mathrm{MHz}, \mathrm{CDCl}_{3}\right)$
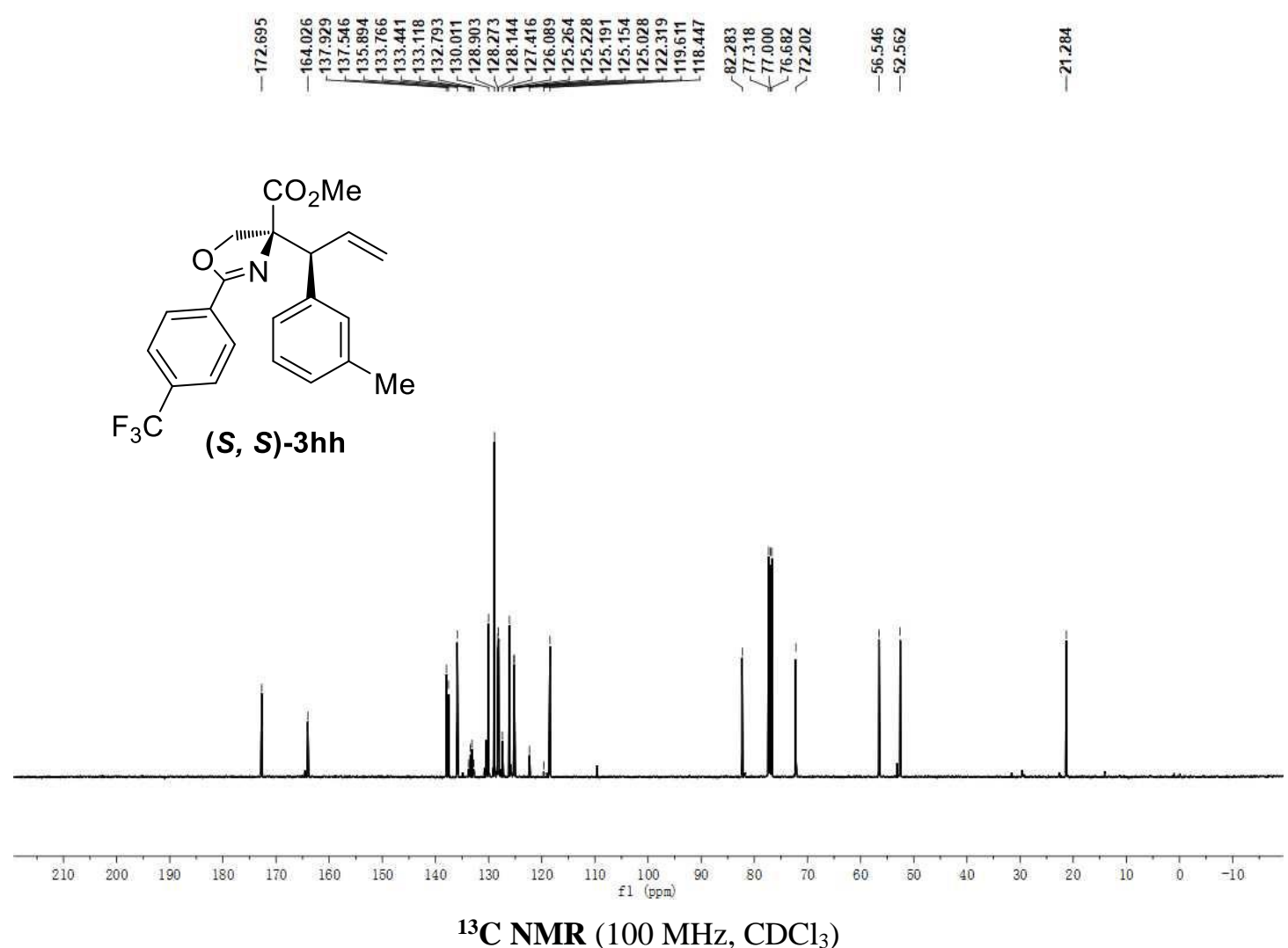

${ }^{13} \mathbf{C}$ NMR $\left(100 \mathrm{MHz}, \mathrm{CDCl}_{3}\right)$ 

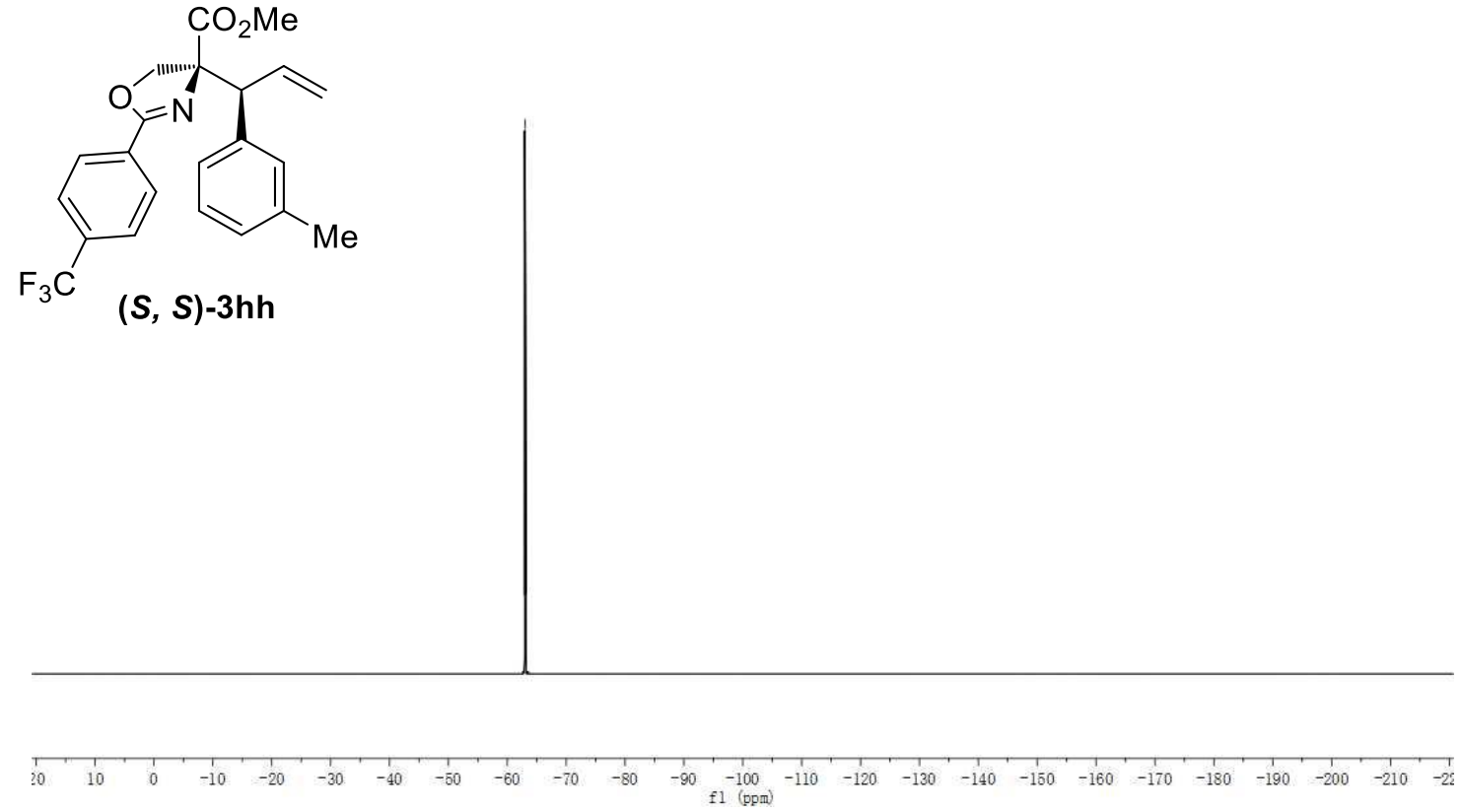

${ }^{19}$ F NMR $\left(376 \mathrm{MHz}, \mathrm{CDCl}_{3}\right)$ 


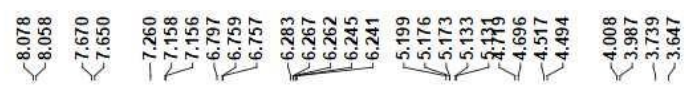
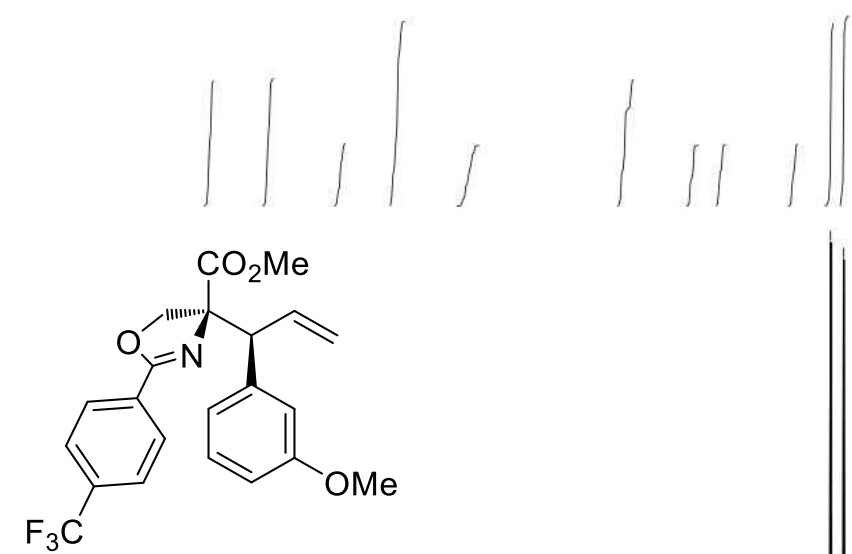

$(S, S)-3 h i$
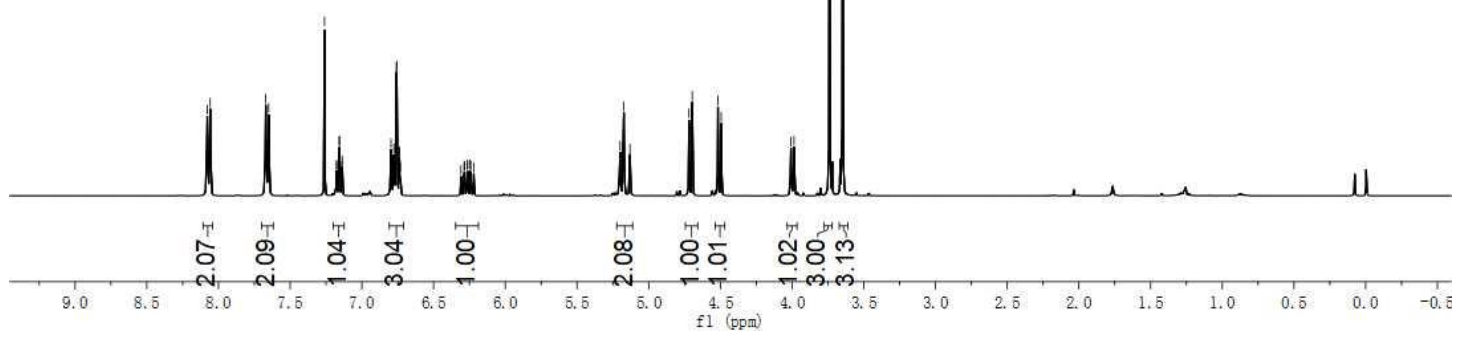

${ }^{1} \mathbf{H}$ NMR $\left(400 \mathrm{MHz}, \mathrm{CDCl}_{3}\right)$

象<smiles>C=CC(c1cccc(OC)c1)C(C)(COC)N1COC(c2ccc(C(F)(F)F)cc2)=C1C</smiles>

$(S, S)-3 h i$

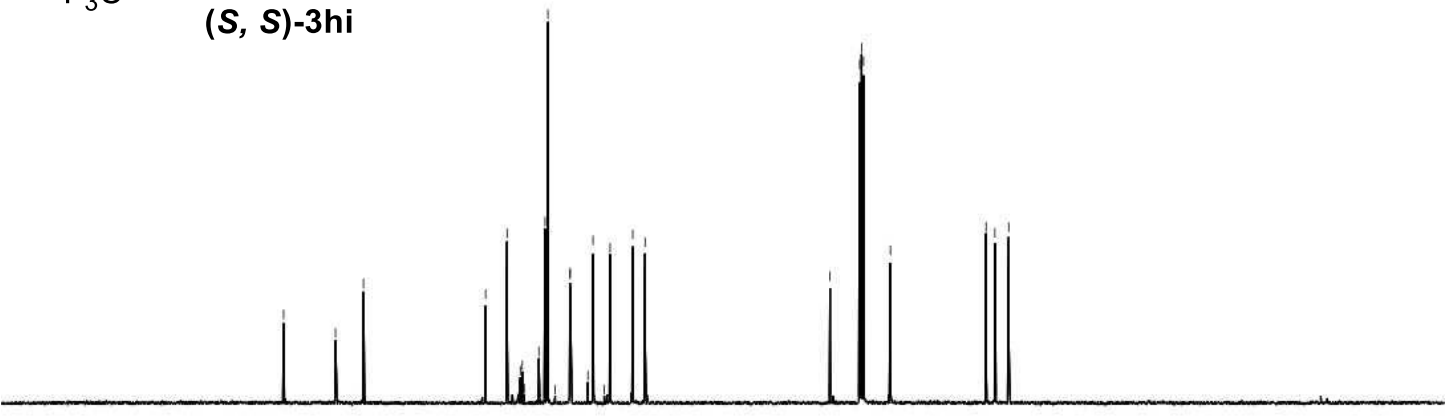

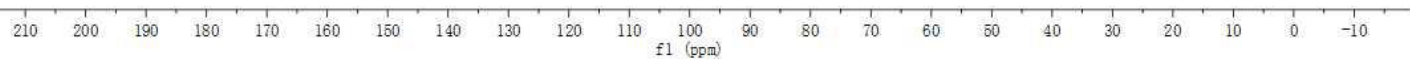

${ }^{13} \mathbf{C}$ NMR $\left(100 \mathrm{MHz}, \mathrm{CDCl}_{3}\right)$ 
<smiles>C=C[C@H](c1cccc(OC)c1)[C@@]1(C(C)=O)COC(c2ccc(C(F)(F)F)cc2)=N1</smiles>

(S, S)-3hi

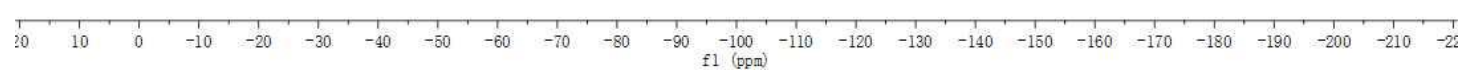

${ }^{19}$ F NMR (376 MHz, $\left.\mathrm{CDCl}_{3}\right)$ 


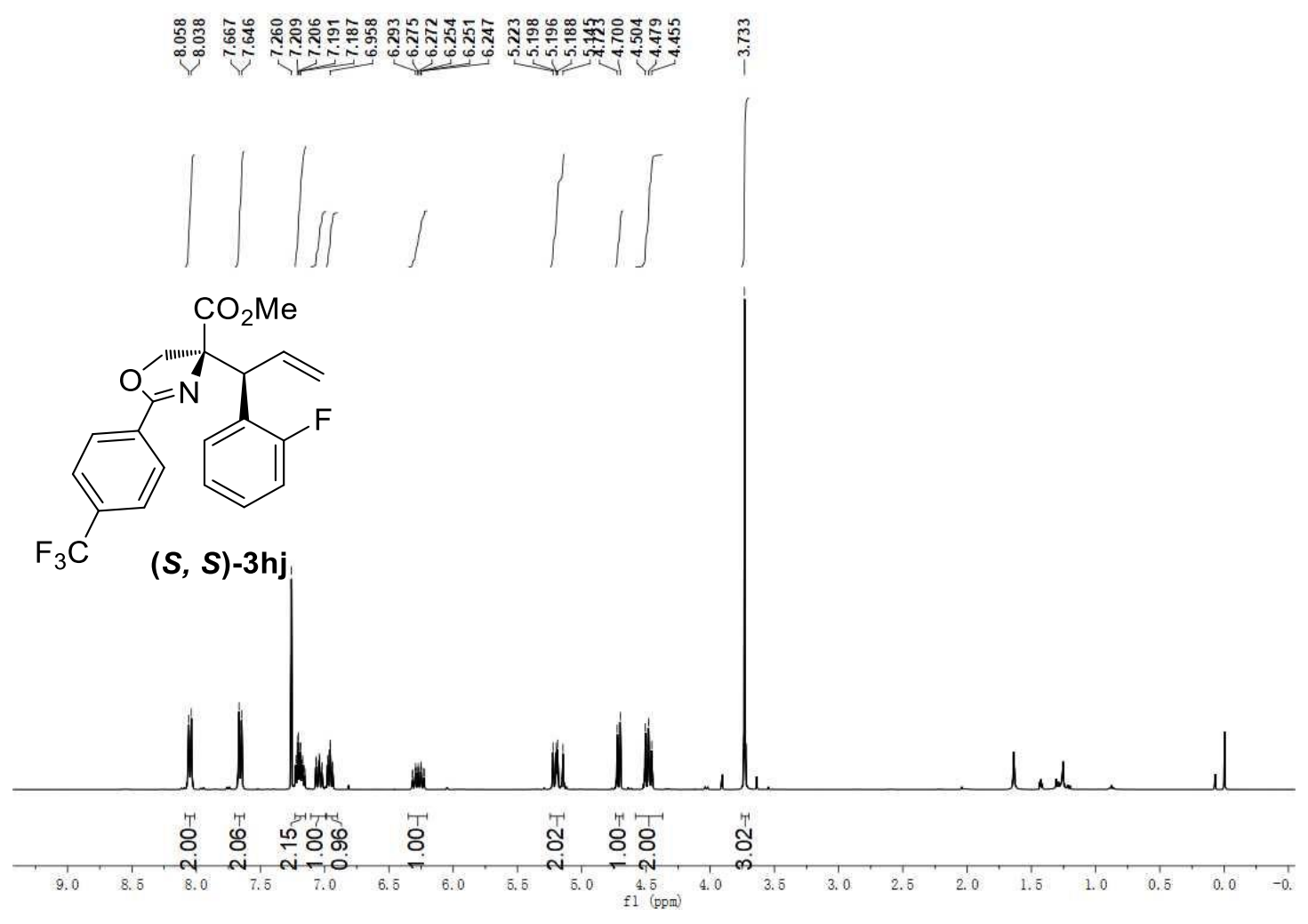

${ }^{1} \mathbf{H}$ NMR $\left(400 \mathrm{MHz}, \mathrm{CDCl}_{3}\right)$

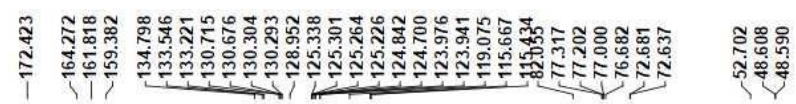
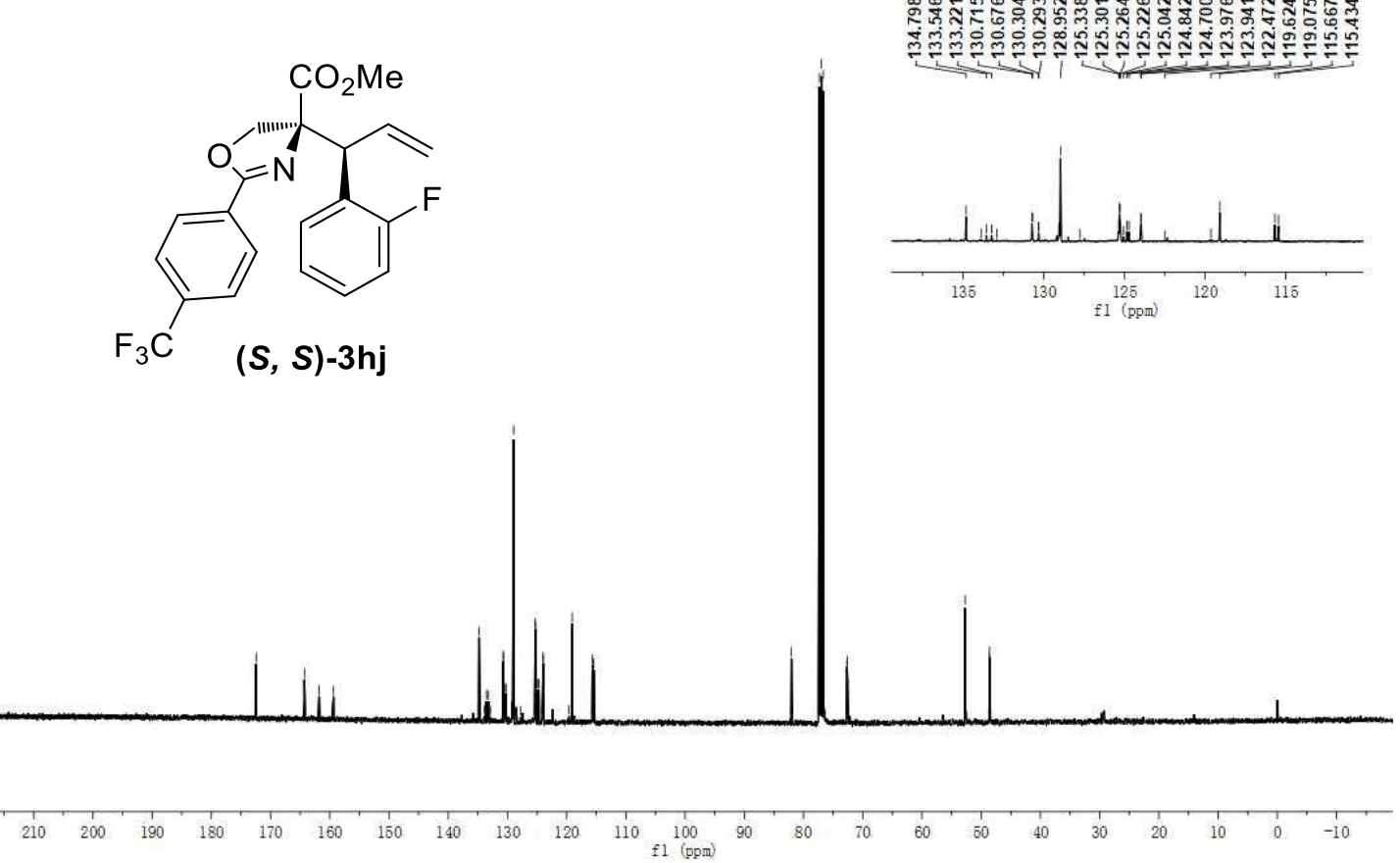

${ }^{13} \mathbf{C}$ NMR $\left(100 \mathrm{MHz}, \mathrm{CDCl}_{3}\right)$ 
<smiles>C=CC(c1ccccc1F)[C@@]1(C(C)=O)COC(c2ccc(C(F)(F)F)cc2)=N1</smiles>

$(S, S)-3 h j$

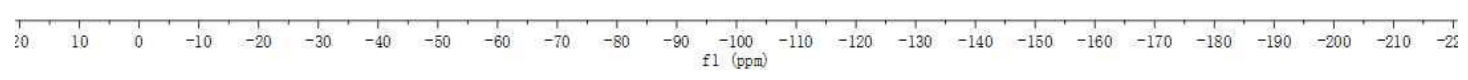

${ }^{19} \mathbf{F}$ NMR $\left(376 \mathrm{MHz}, \mathrm{CDCl}_{3}\right)$ 


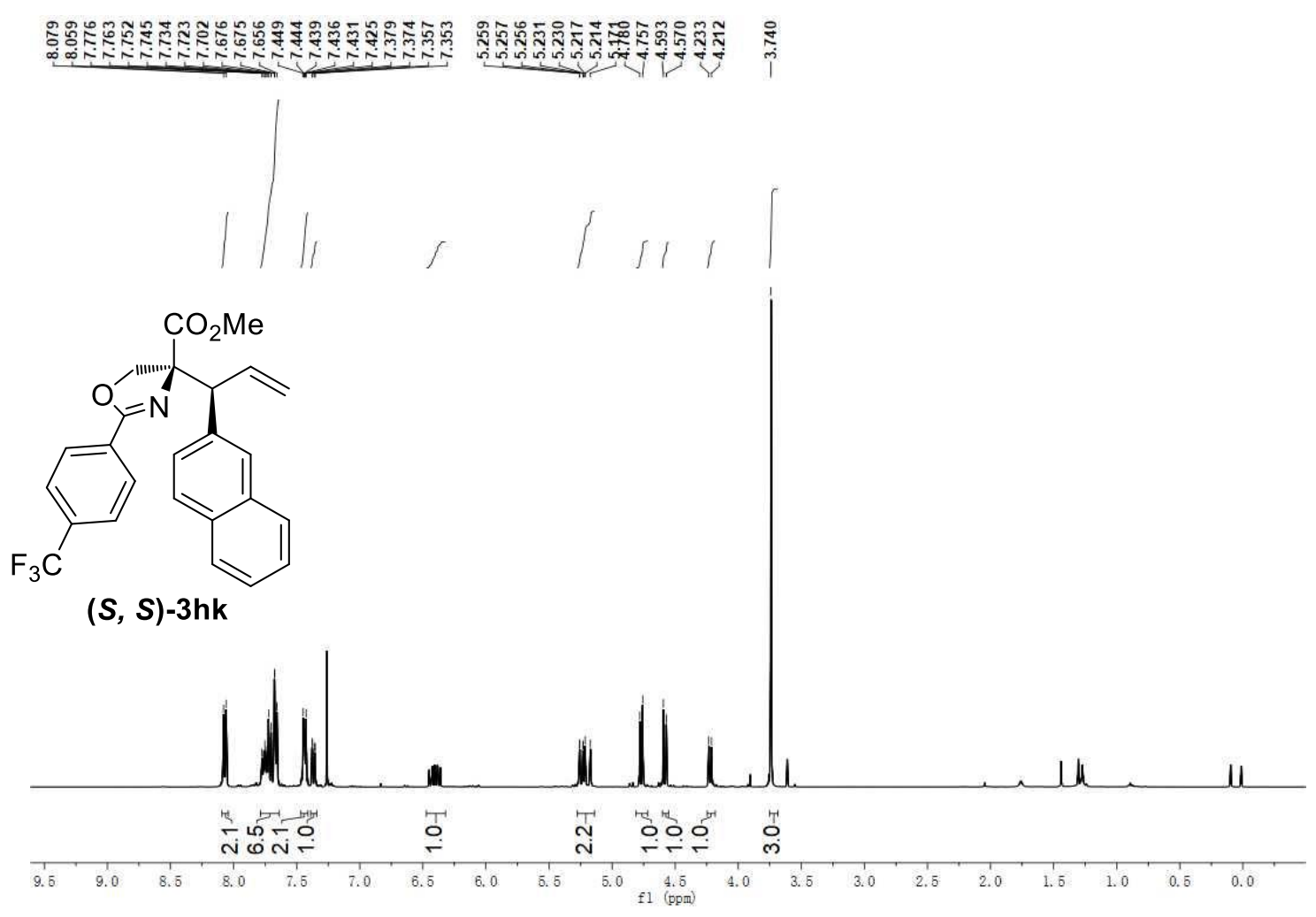

${ }^{1} \mathbf{H}$ NMR $\left(400 \mathrm{MHz}, \mathrm{CDCl}_{3}\right)$
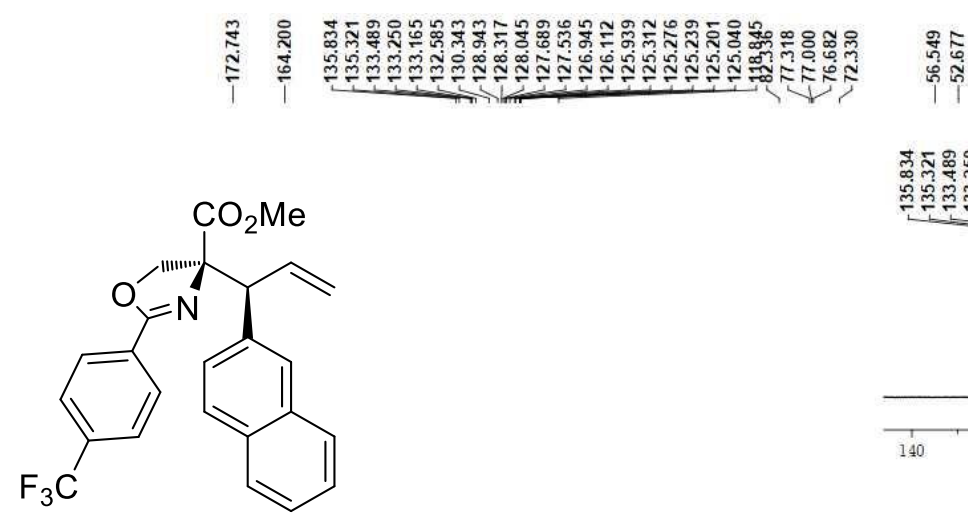

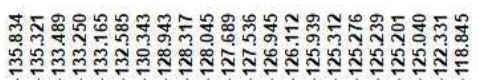

$(S, S)-3 \mathrm{hk}$
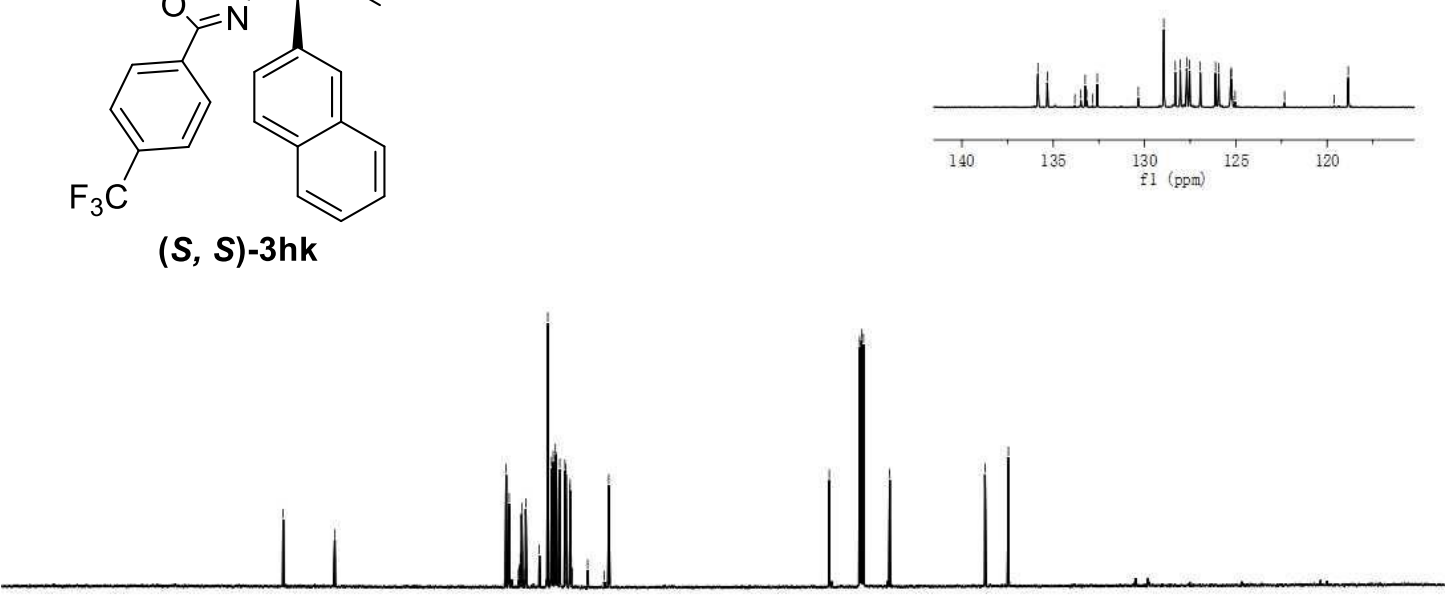

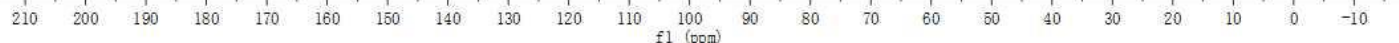

${ }^{13}$ C NMR (100 MHz, $\left.\mathrm{CDCl}_{3}\right)$ 
ֻ屯<smiles>C=CC(c1ccc2ccccc2c1)C(C)(NC(=O)c1ccc(C(F)(F)F)cc1)C(C)=O</smiles>

(S, S)-3hk

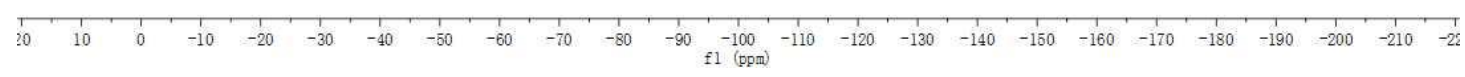

${ }^{19} \mathbf{F}$ NMR $\left(376 \mathrm{MHz}, \mathrm{CDCl}_{3}\right)$ 


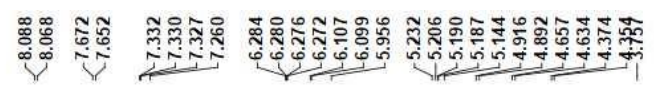
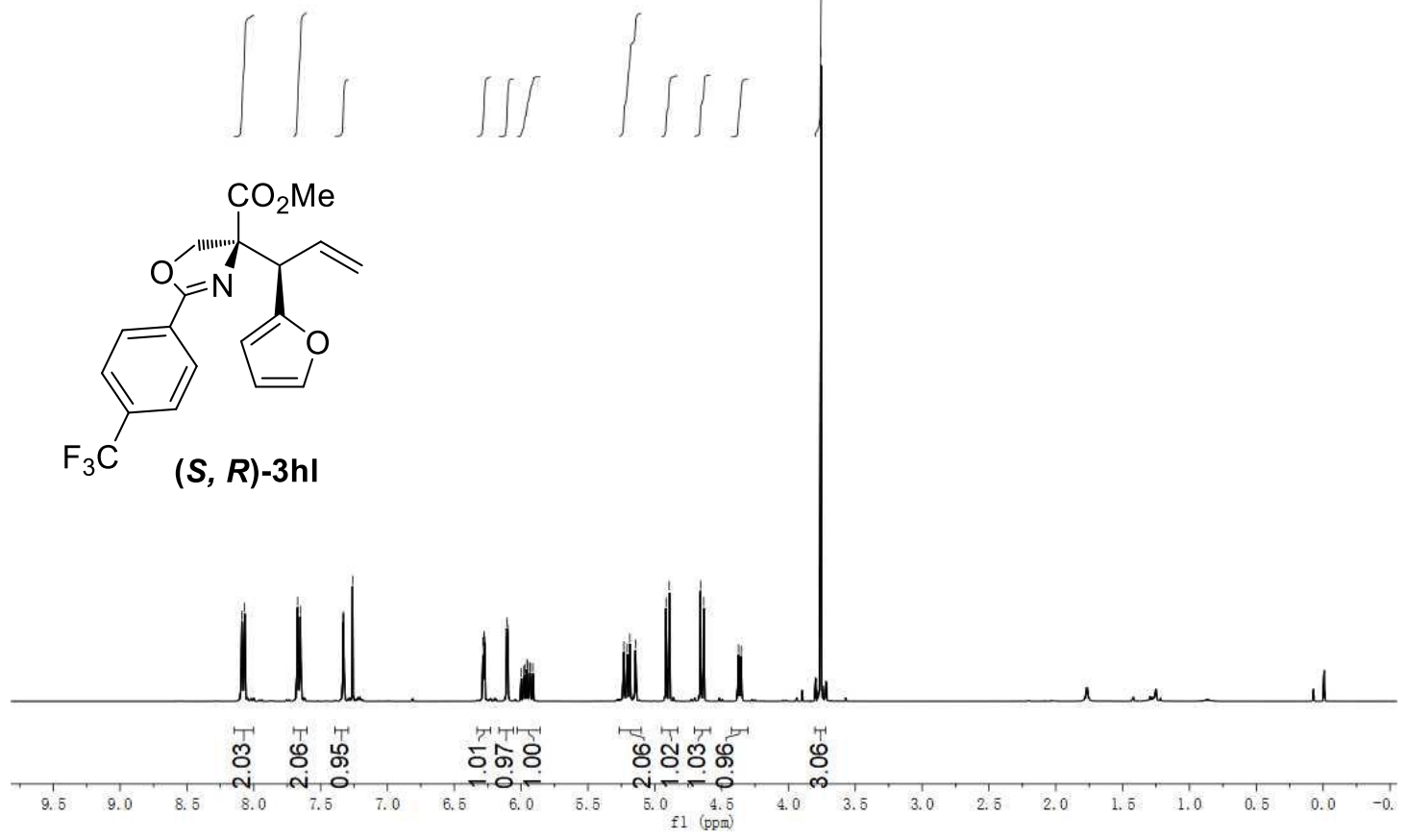

${ }^{1} \mathbf{H}$ NMR $\left(400 \mathrm{MHz}, \mathrm{CDCl}_{3}\right)$
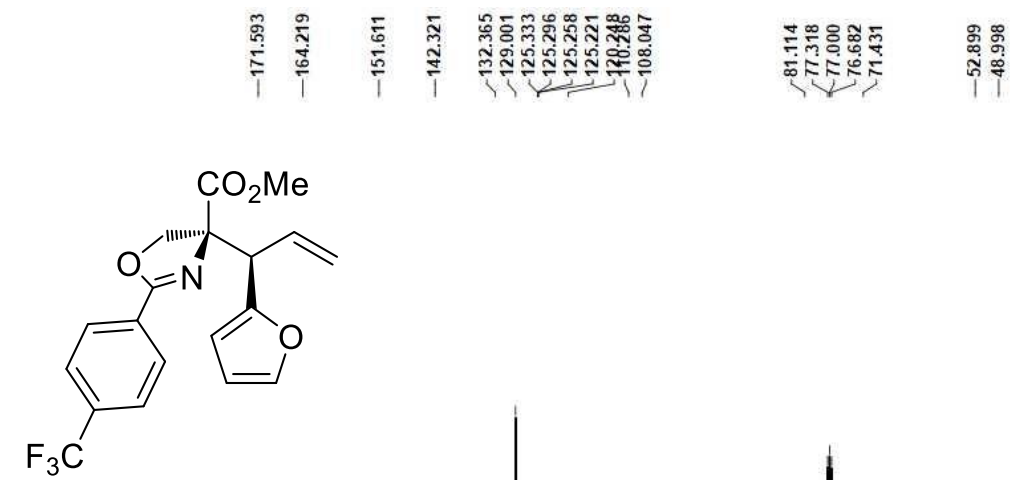

$(S, R)-3 \mathrm{hl}$

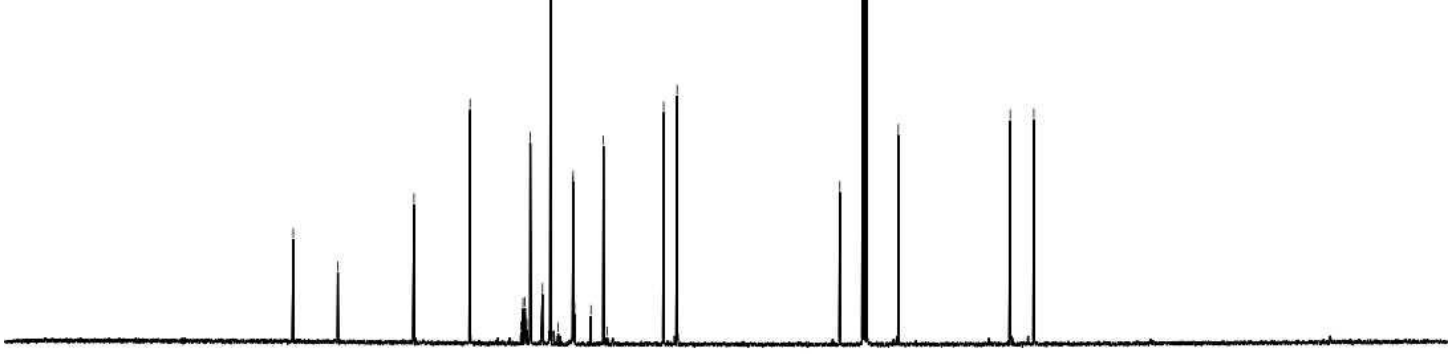

$\begin{array}{lllllllllllllllllllllllllllllllllllll}210 & 200 & 190 & 180 & 170 & 160 & 150 & 140 & 130 & 120 & 110 & 100 & 90 & 80 & 70 & 60 & 50 & 40 & 30 & 20 & 10 & 0 & -10\end{array}$

${ }^{13}$ C NMR (100 MHz, $\left.\mathrm{CDCl}_{3}\right)$ 

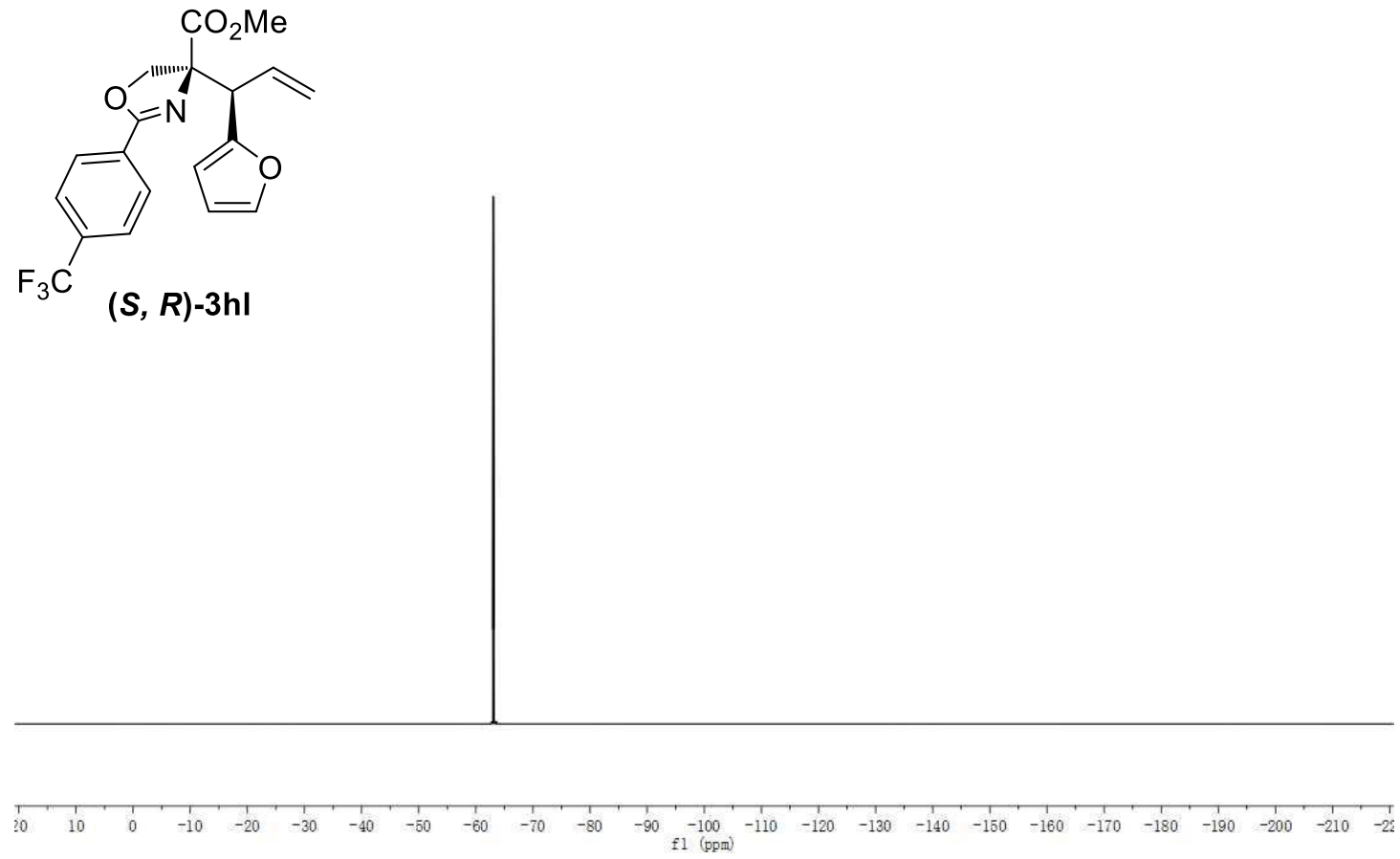

${ }^{19} \mathbf{F}$ NMR $\left(376 \mathrm{MHz}, \mathrm{CDCl}_{3}\right)$ 

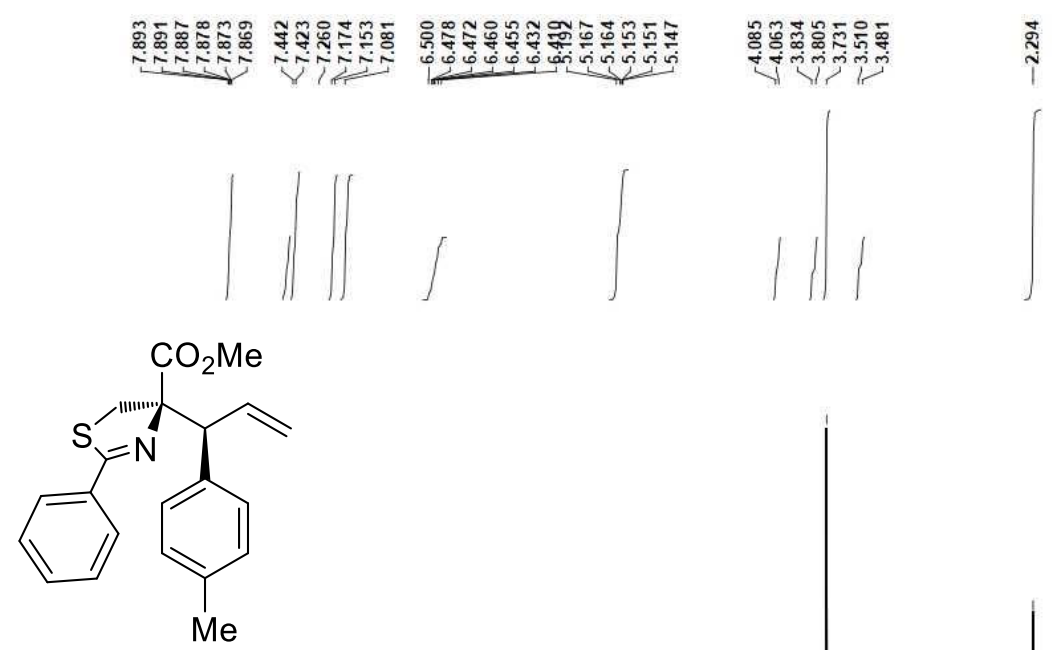

$(R, S)$-3ib

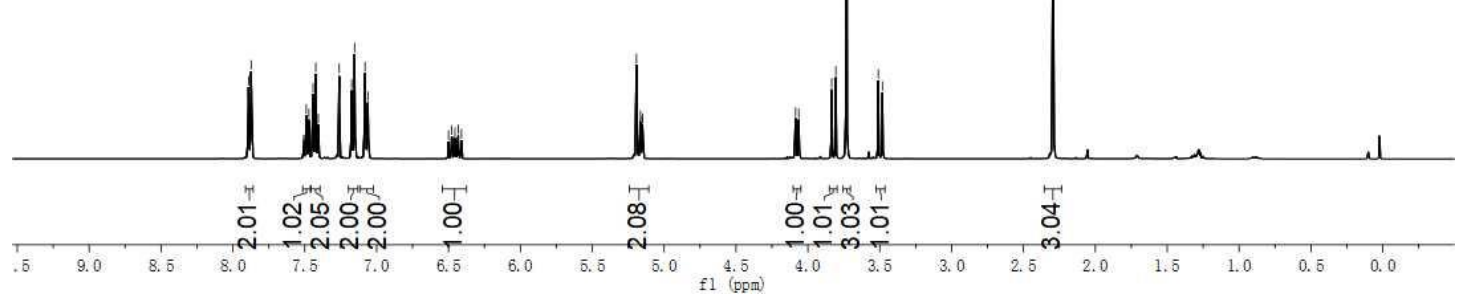

${ }^{1} \mathbf{H}$ NMR $\left(400 \mathrm{MHz}, \mathrm{CDCl}_{3}\right)$
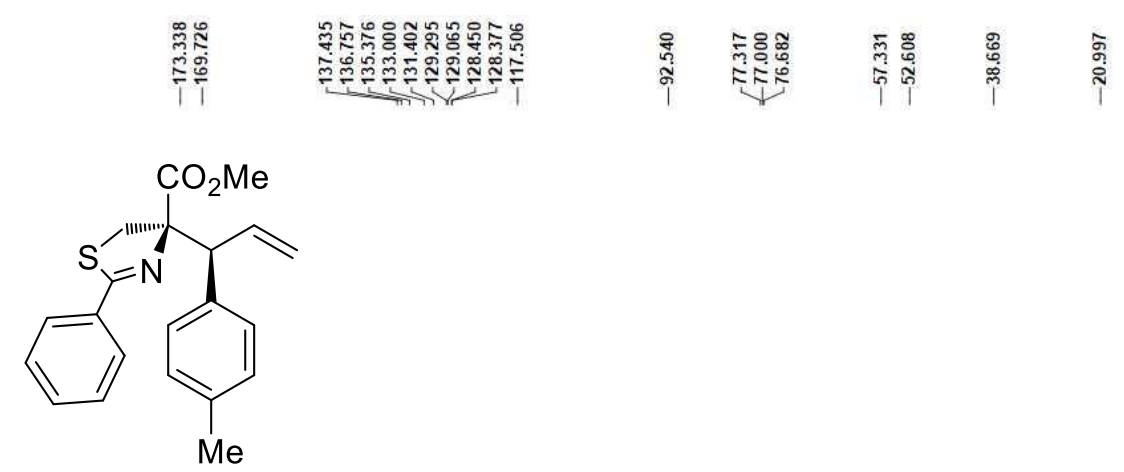

$(R, S)-3 i b$
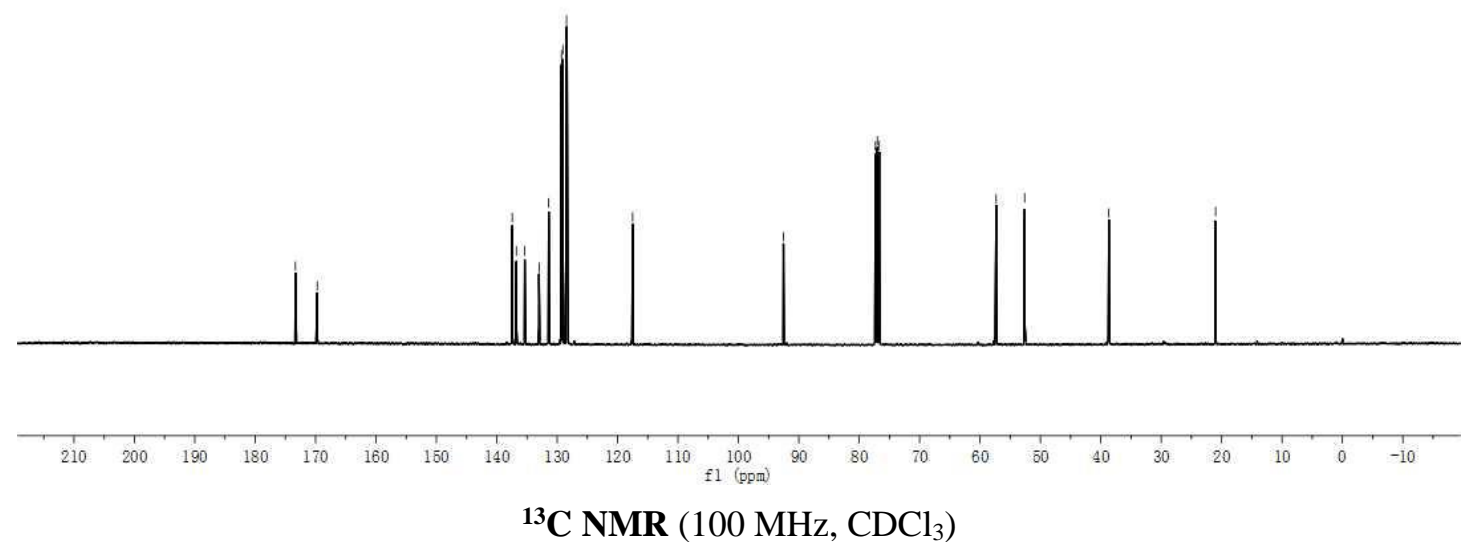


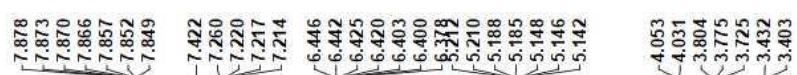
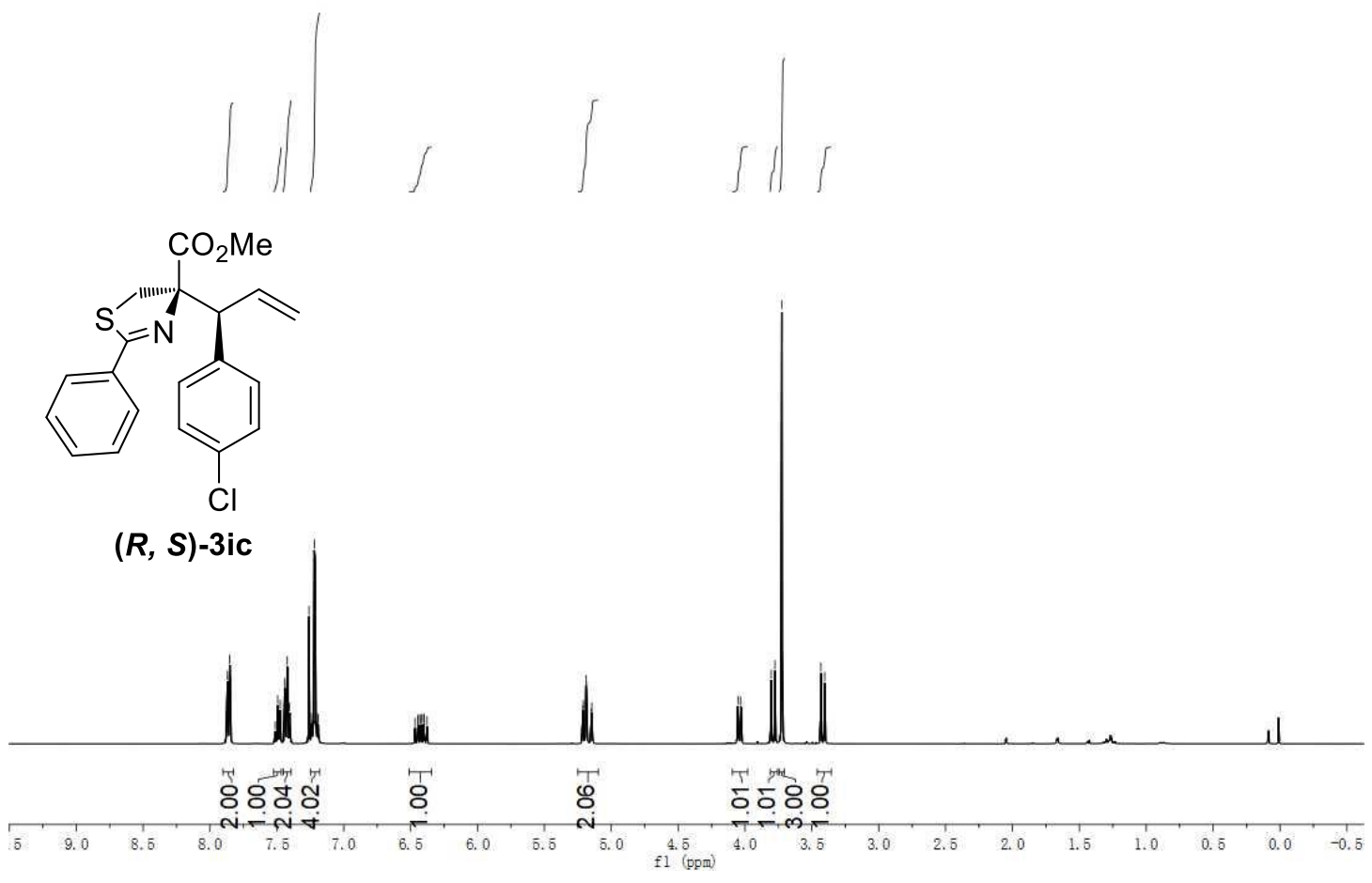

${ }^{1} \mathbf{H}$ NMR $\left(400 \mathrm{MHz}, \mathrm{CDCl}_{3}\right)$

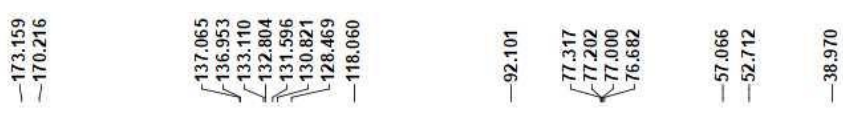

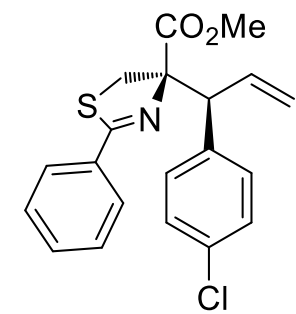

$(R, S)-3 i c$
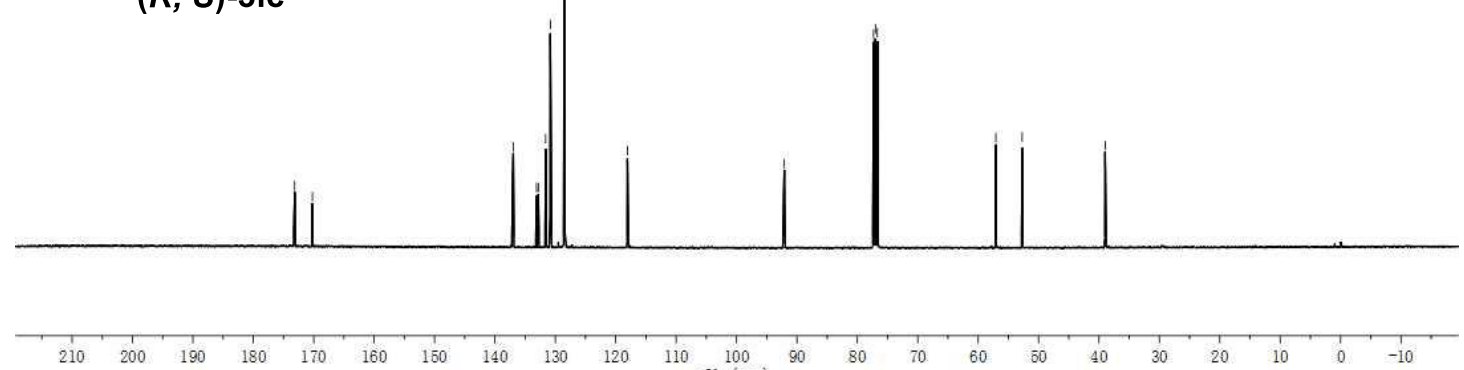

${ }^{13}$ C NMR (100 MHz, $\left.\mathrm{CDCl}_{3}\right)$ 


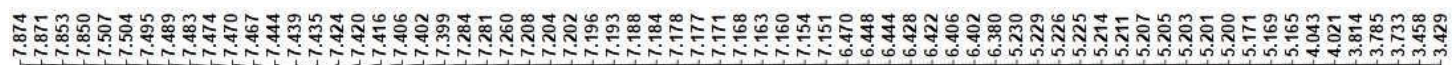
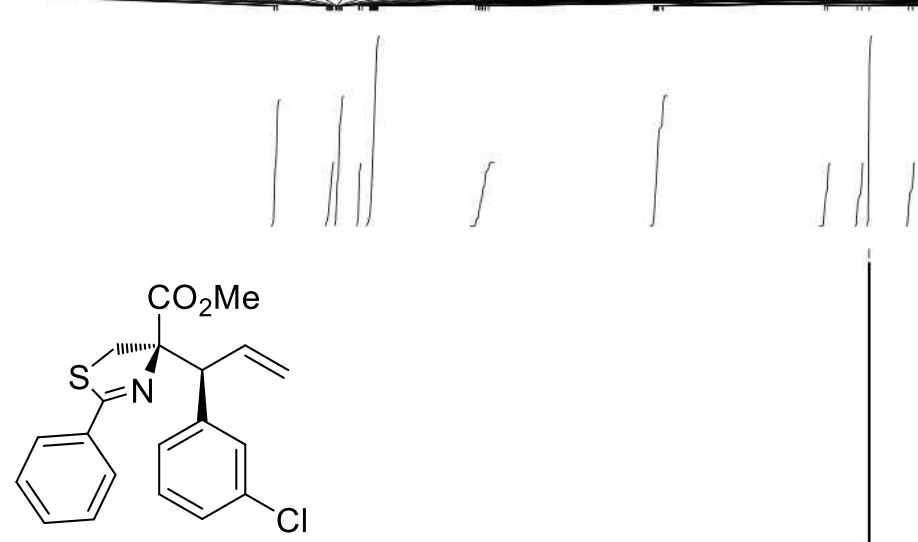

$(R, S)-3 i g$
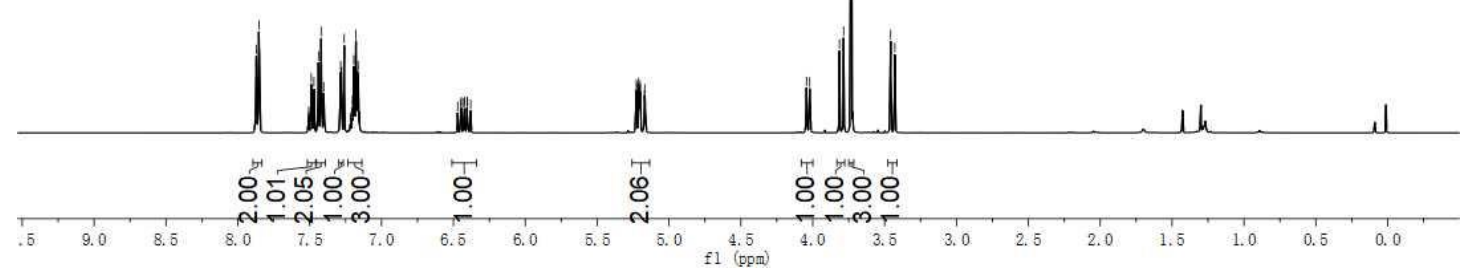

${ }^{1} \mathbf{H}$ NMR $\left(400 \mathrm{MHz}, \mathrm{CDCl}_{3}\right)$

สิ๊

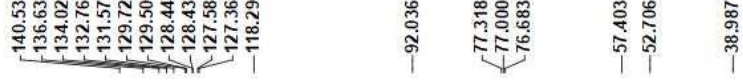

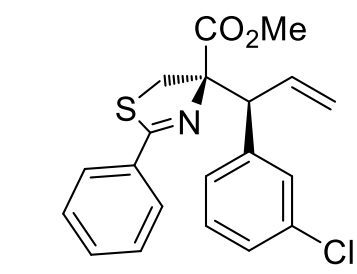

$(R, S)-3 i g$
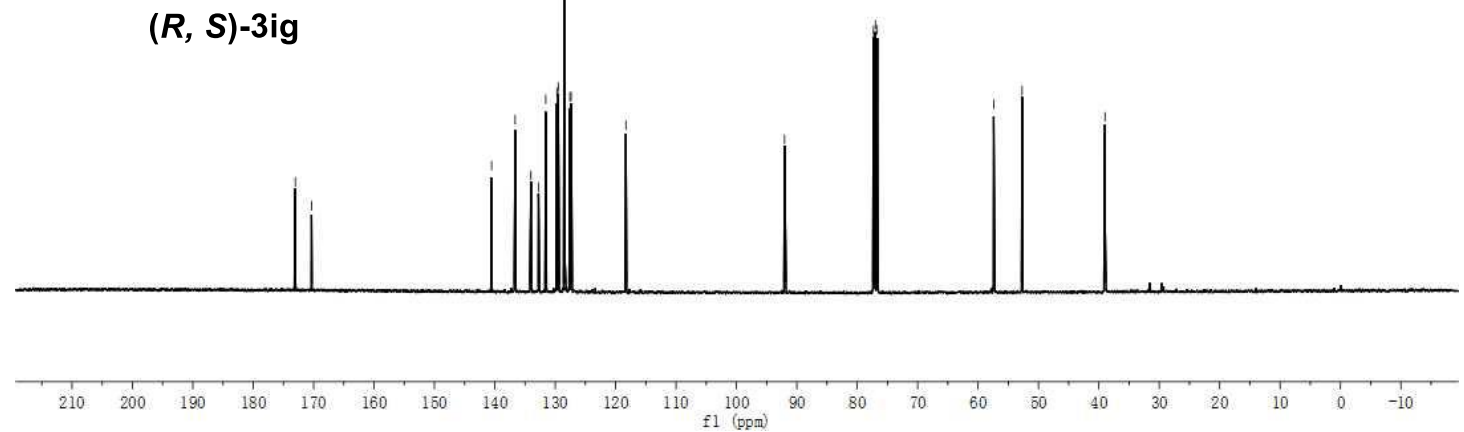

${ }^{13} \mathbf{C}$ NMR $\left(100 \mathrm{MHz}, \mathrm{CDCl}_{3}\right)$ 


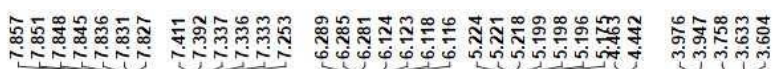
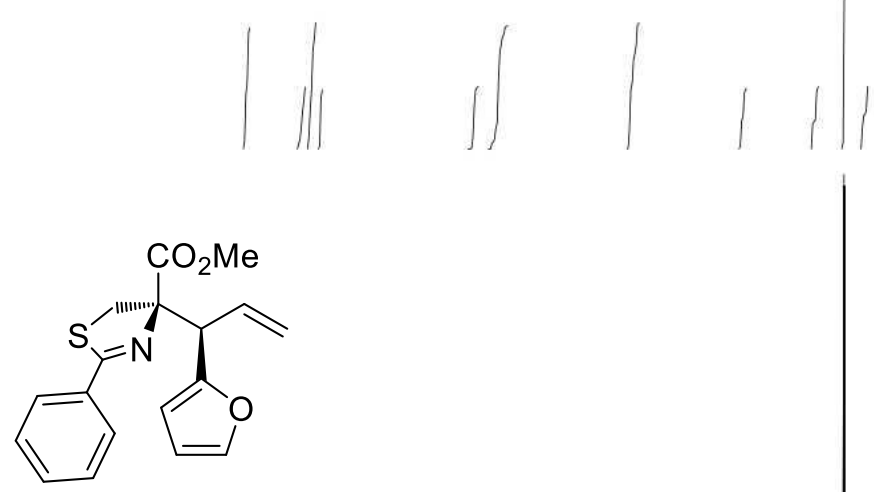

$(R, R)-3$ il

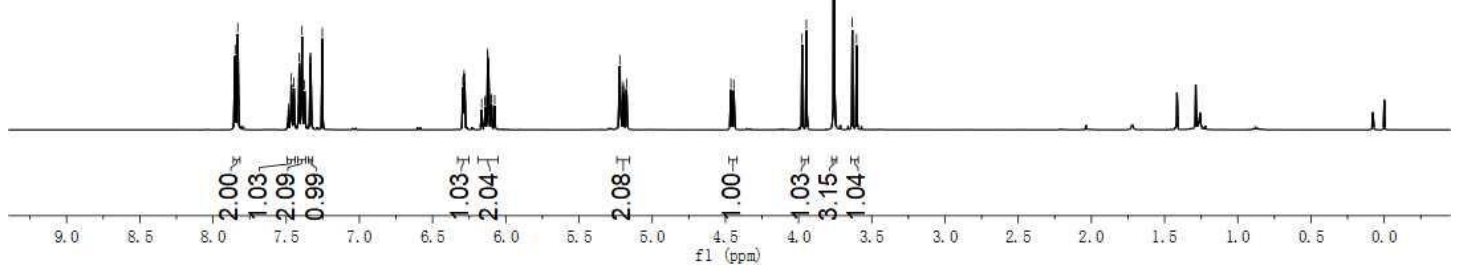

${ }^{1} \mathbf{H}$ NMR $\left(400 \mathrm{MHz}, \mathrm{CDCl}_{3}\right)$

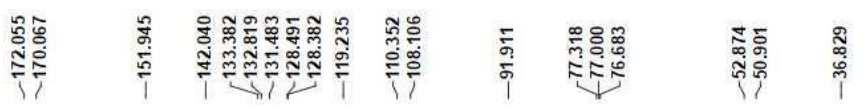

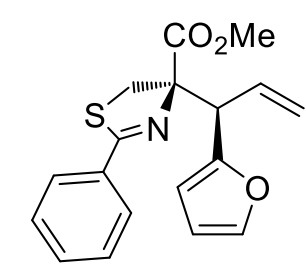

$(R, R)-3$ il
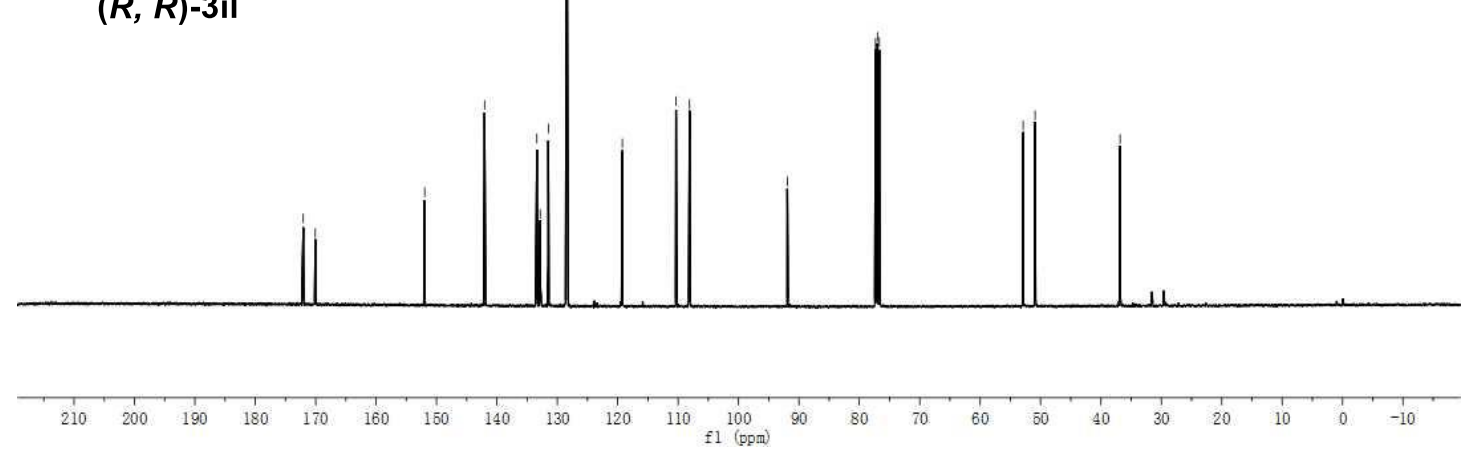

${ }^{13} \mathbf{C}$ NMR $\left(100 \mathrm{MHz}, \mathrm{CDCl}_{3}\right)$ 


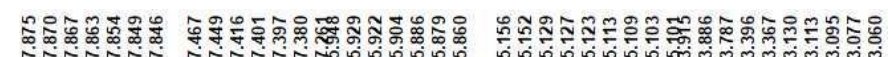

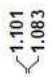

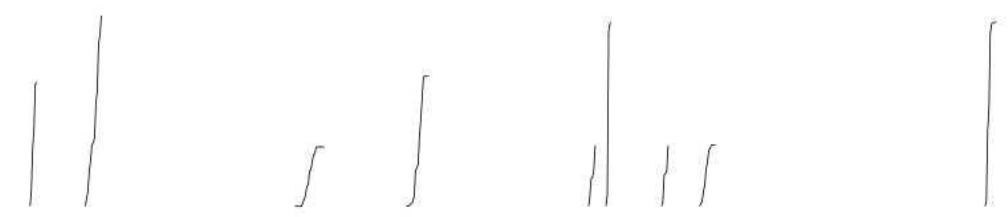

$>_{(R, R)-3 \mathrm{im}}^{\mathrm{CO}_{2} \mathrm{Me}}$

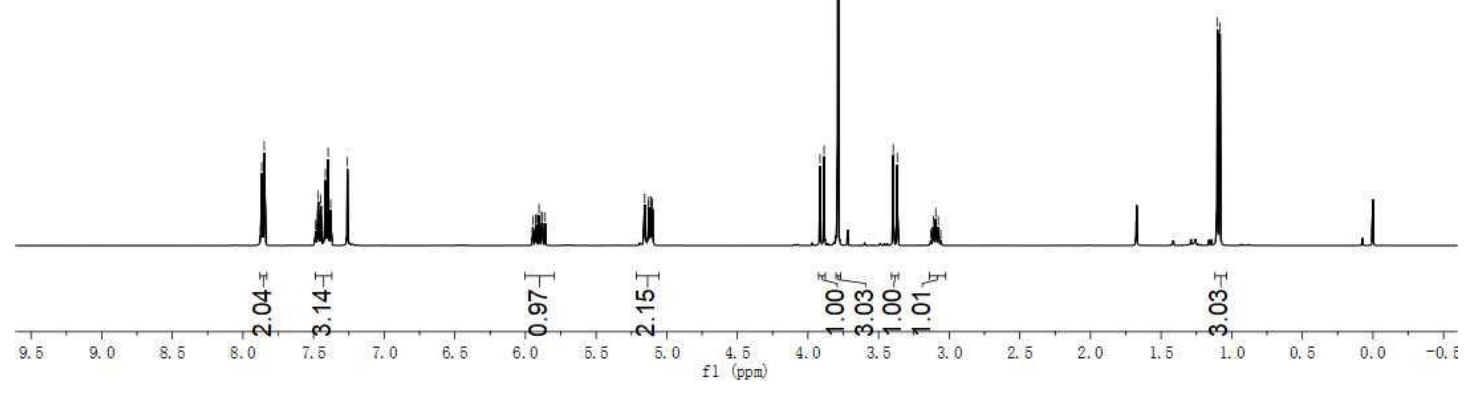

${ }^{1} \mathbf{H}$ NMR $\left(400 \mathrm{MHz}, \mathrm{CDCl}_{3}\right)$

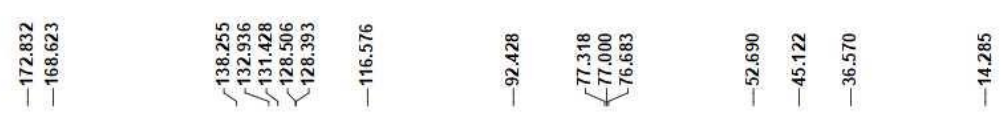
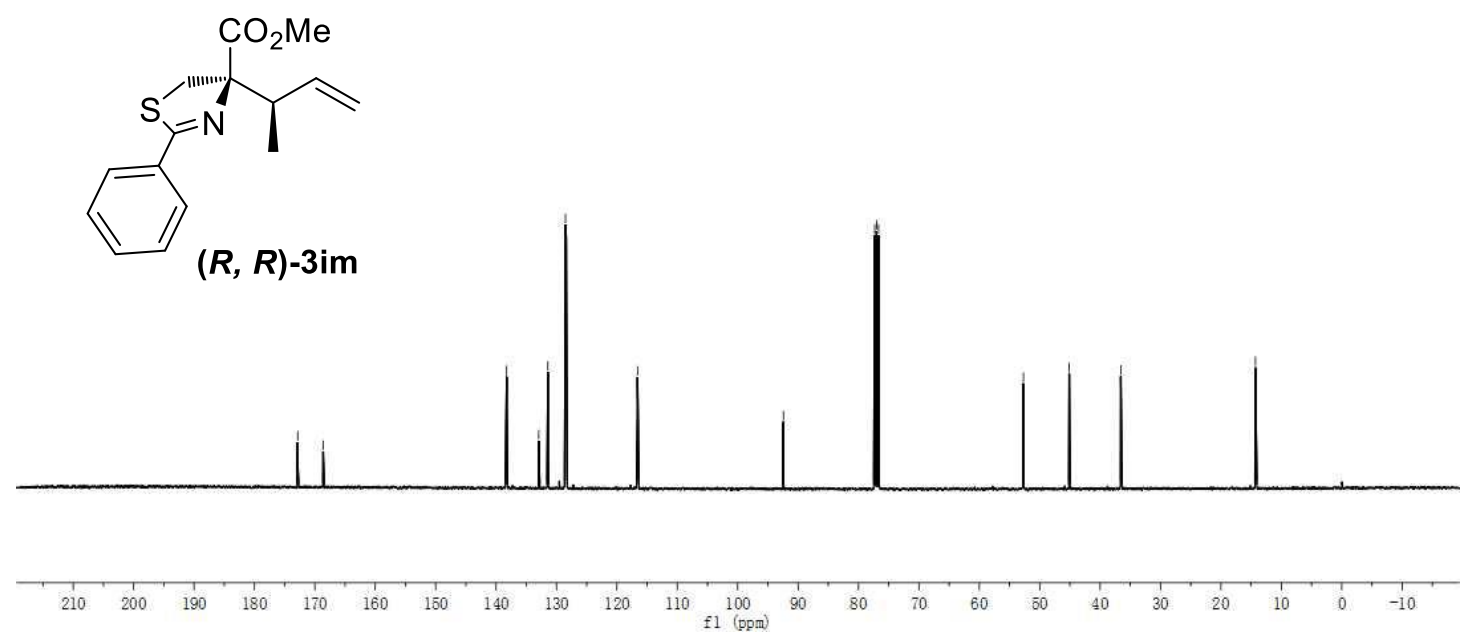

${ }^{13} \mathbf{C}$ NMR $\left(100 \mathrm{MHz}, \mathrm{CDCl}_{3}\right)$ 


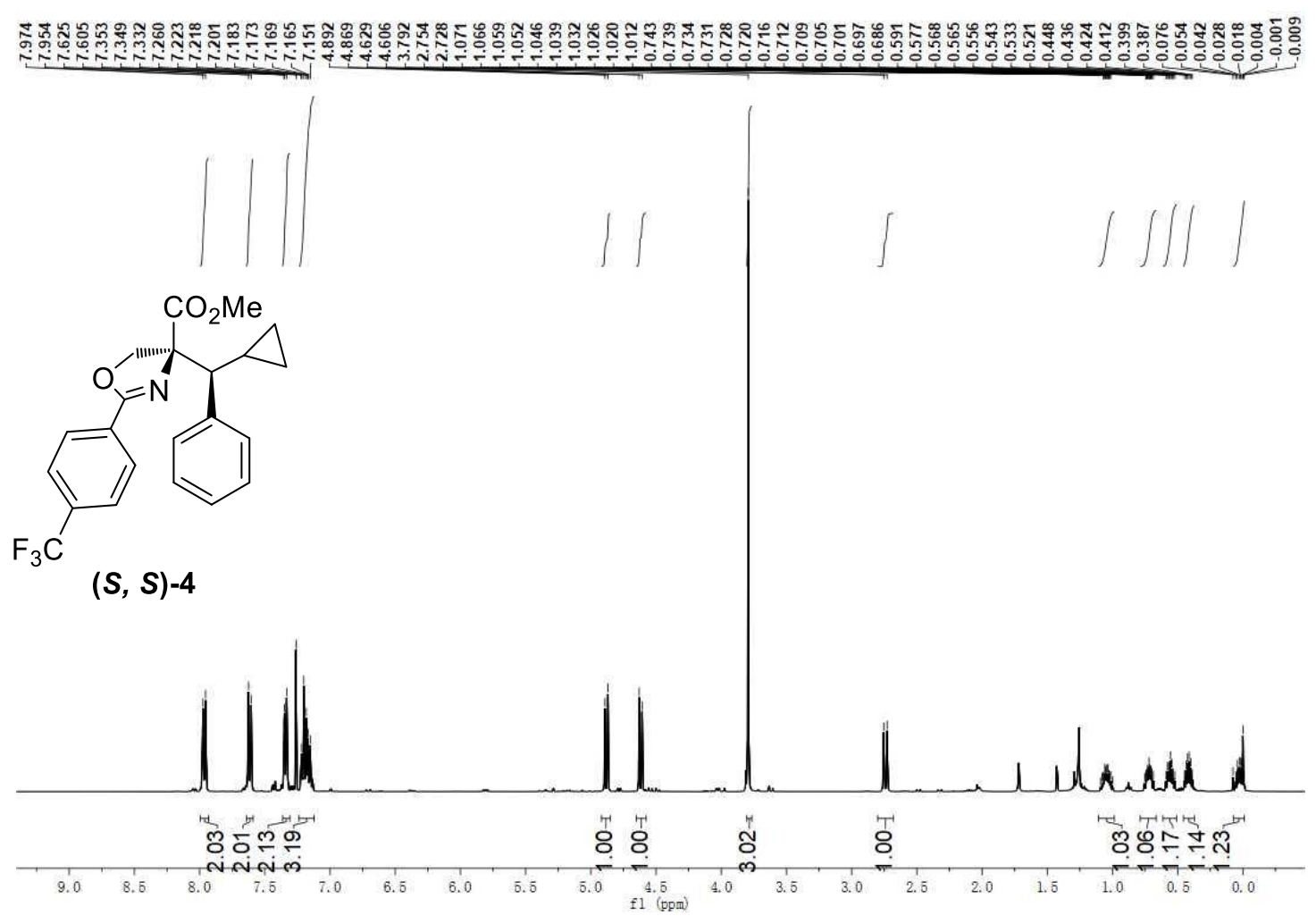

${ }^{1} \mathbf{H}$ NMR $\left(400 \mathrm{MHz}, \mathrm{CDCl}_{3}\right)$
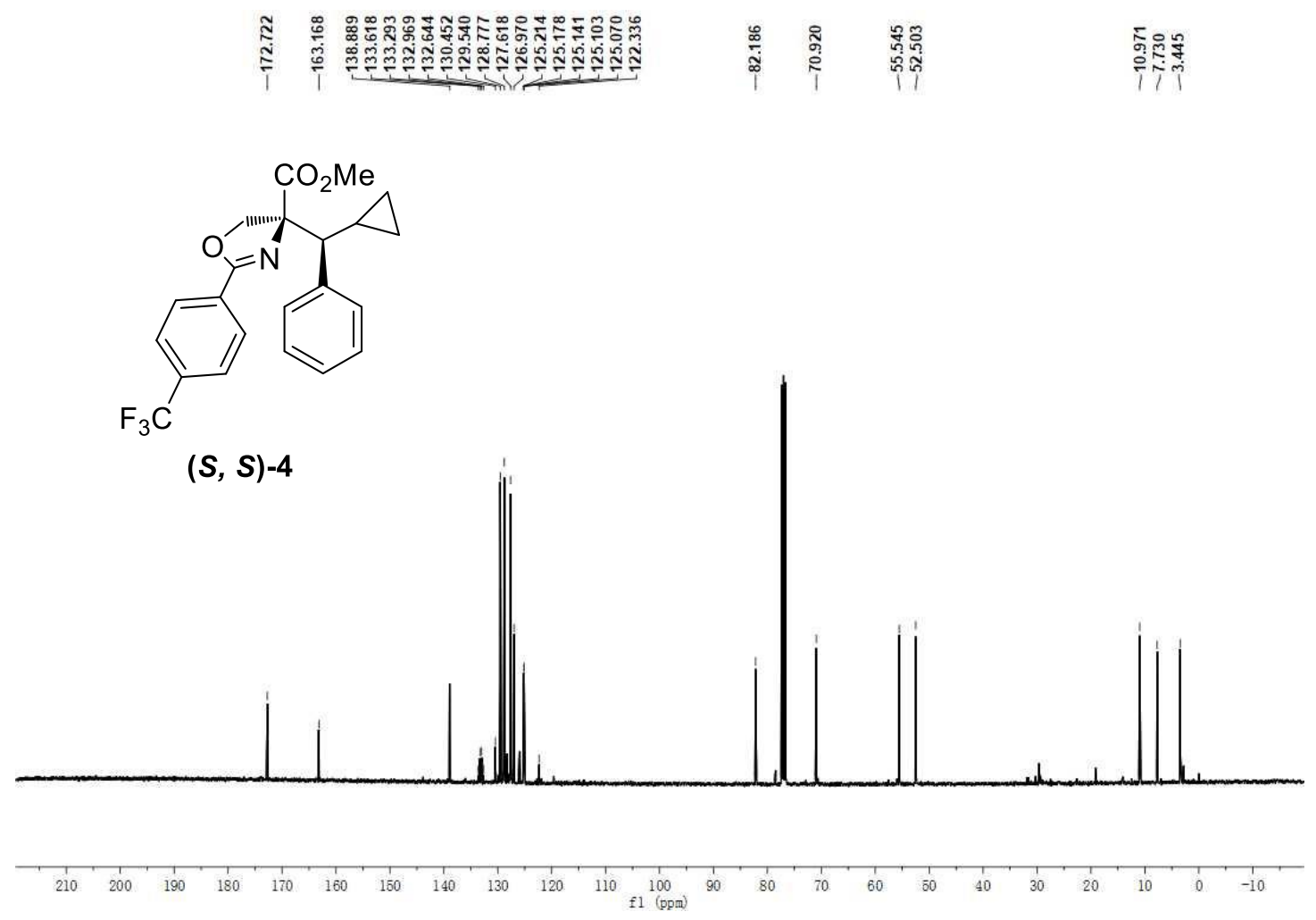

${ }^{13}$ C NMR (100 MHz, $\left.\mathrm{CDCl}_{3}\right)$ 


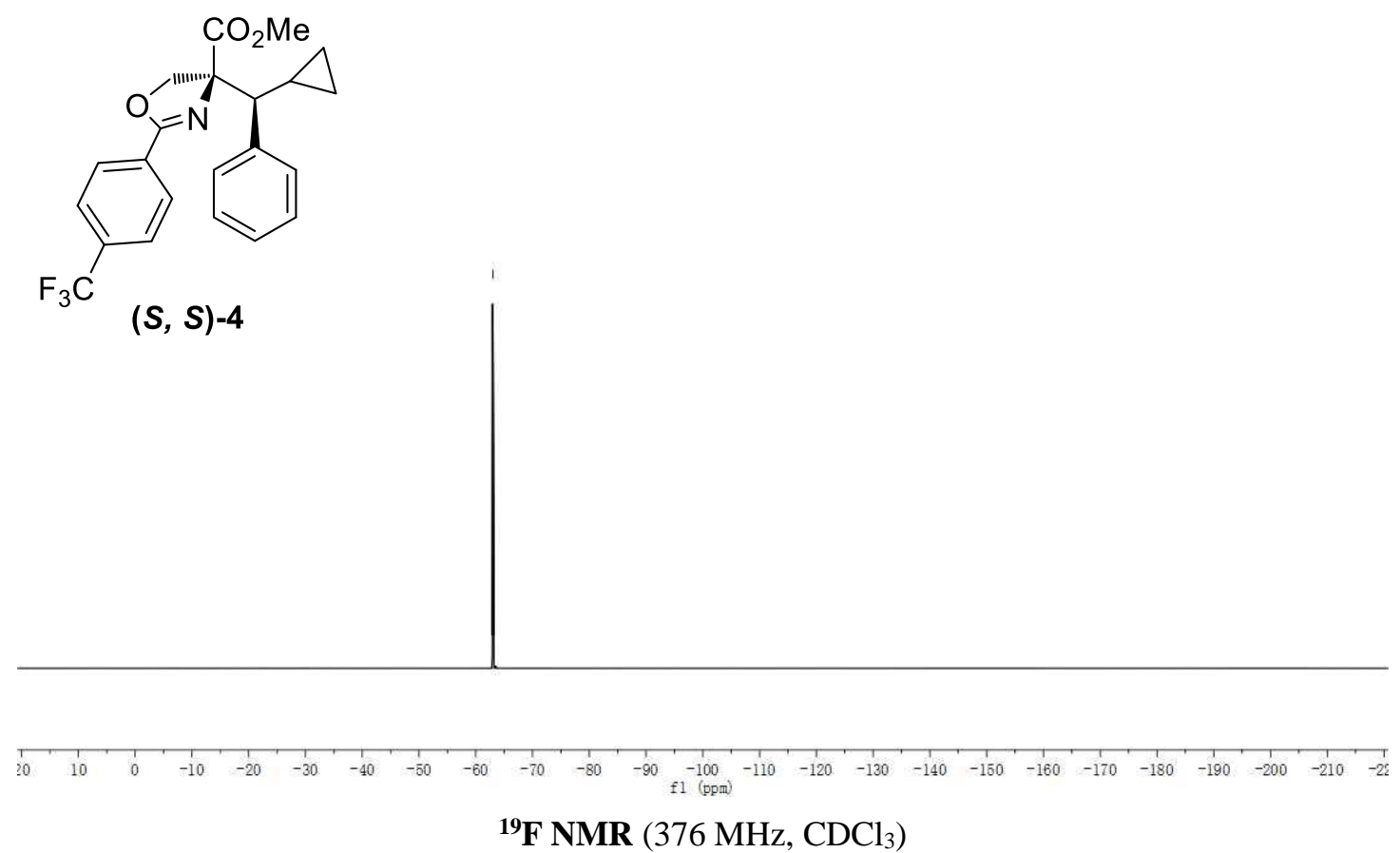




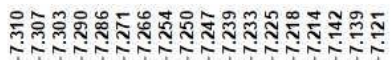

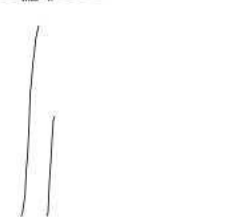

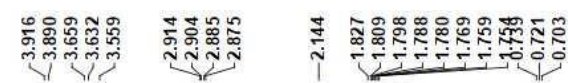

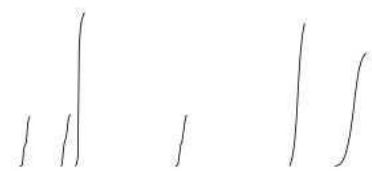<smiles>CCC(c1ccccc1)C(N)(CO)C(C)=O</smiles>

$(S, S)-5$

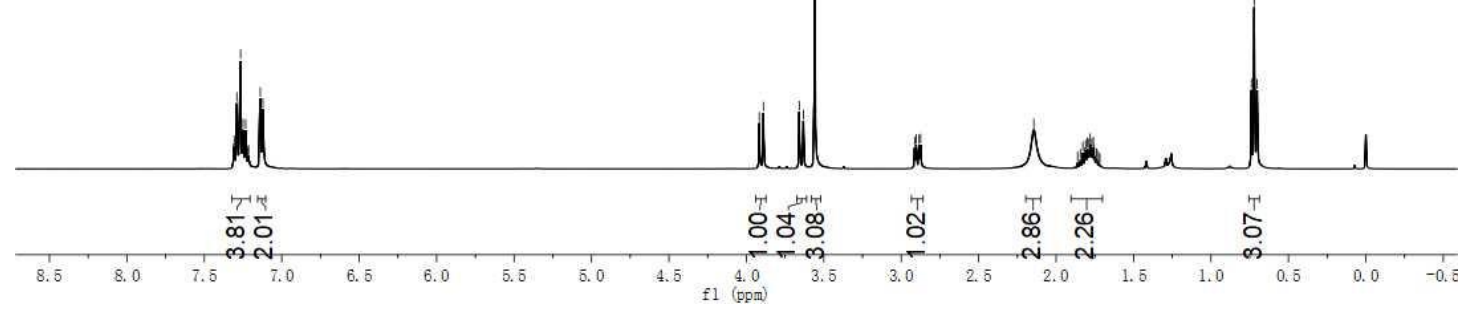

${ }^{1} \mathbf{H}$ NMR $\left(400 \mathrm{MHz}, \mathrm{CDCl}_{3}\right)$

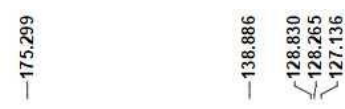

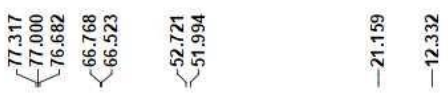

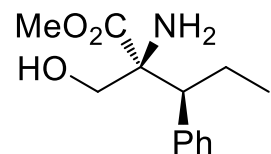

$(S, S)-5$
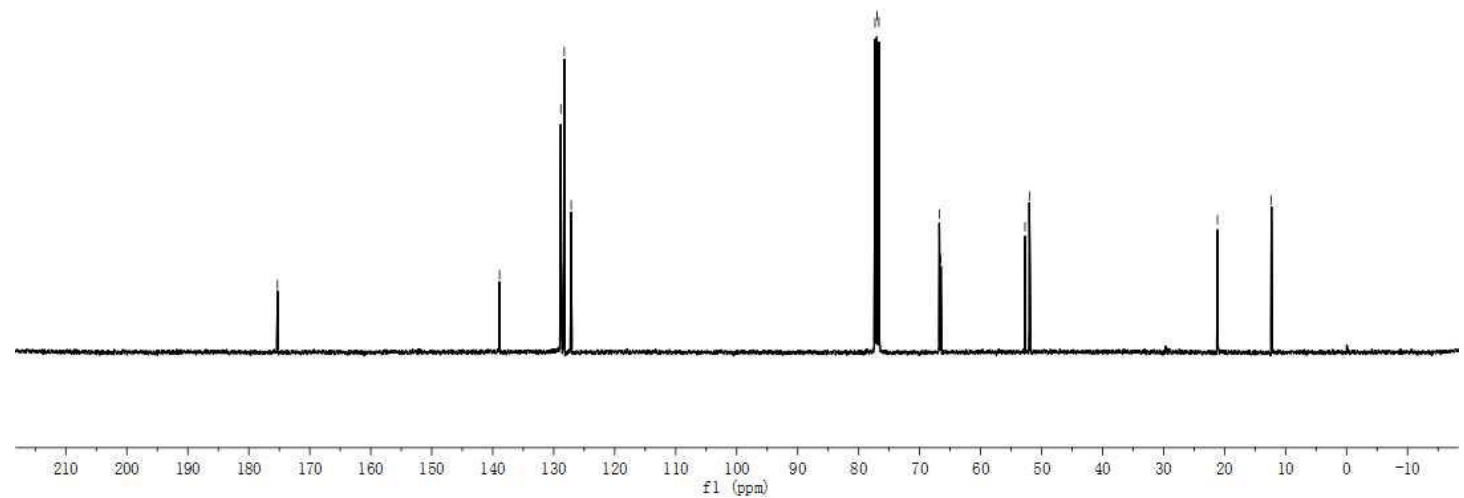

${ }^{13} \mathbf{C}$ NMR $\left(100 \mathrm{MHz}, \mathrm{CDCl}_{3}\right)$ 


\section{HPLC Spectra}<smiles>C=CC(c1ccccc1)[C@@]1(C(C)=O)COC(c2ccccc2)N1</smiles>

\section{racemic-3aa}

Data file E: \DATA WHM \WHM-2-105-RAC 2019-07-20 17-24-50\WHM-2-105-RAC1.D Sample Name: WHM-2-105-RAC
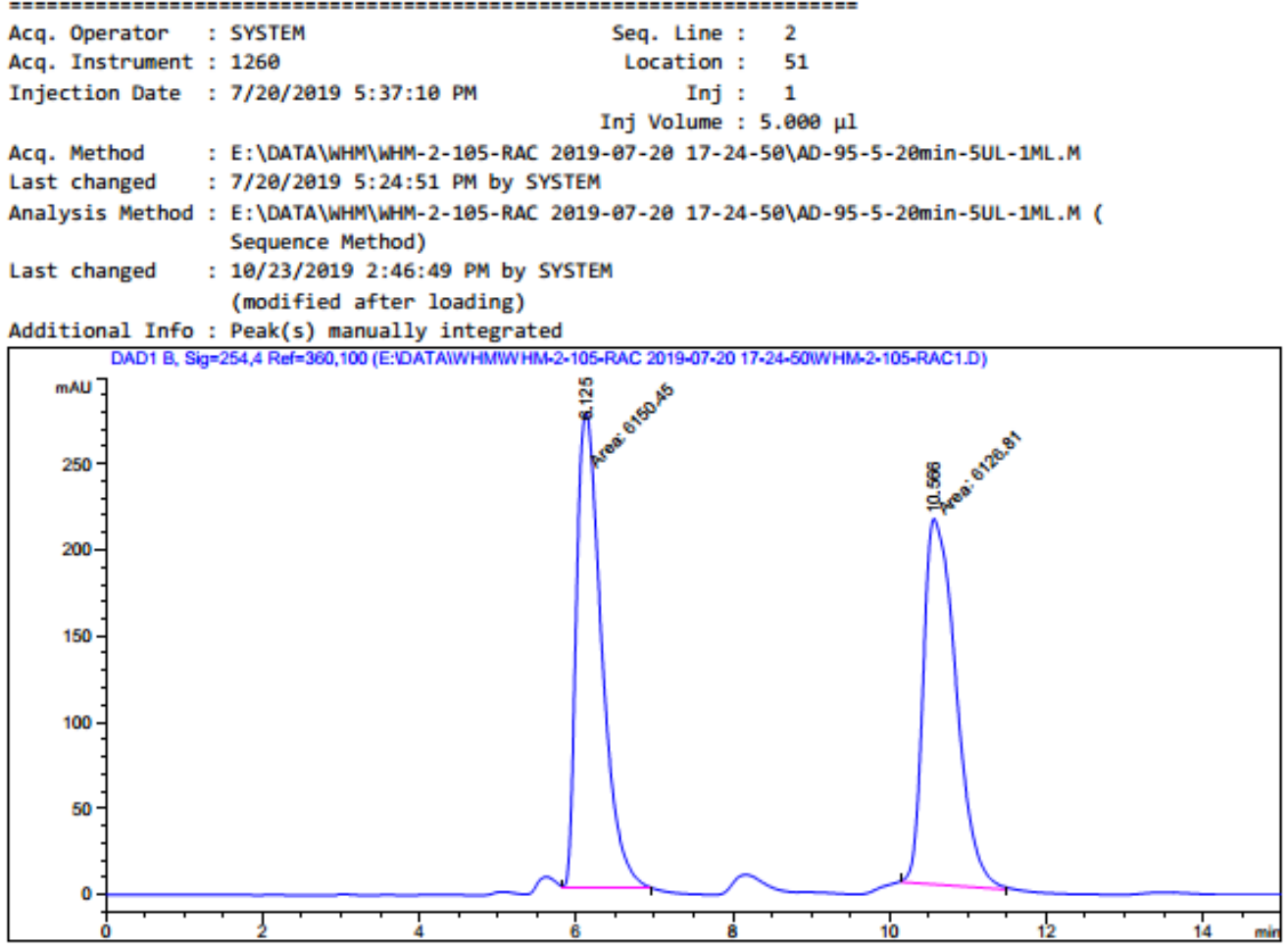

Area Percent Report

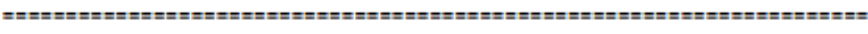

$\begin{array}{lll}\text { Sorted By } & : & \text { Signal } \\ \text { Multiplier } & : & 1.0000\end{array}$

Dilution : 1.000

Do not use Multiplier \& Dilution Factor with ISTDs

Signal 1: DAD1 B, Sig $=254,4$ Ref $=360,100$

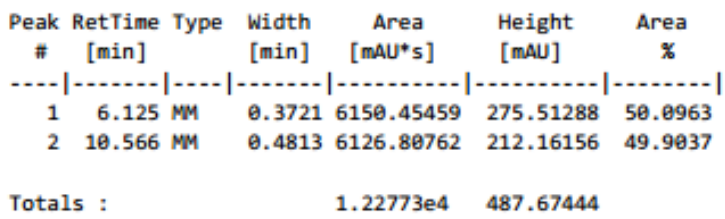


<smiles>C=CC(c1ccccc1)[C@@]1(C(C)=O)COC(c2ccccc2)N1</smiles>

\section{$(S, S)-3 a a$}

Data File E: \DATA\WHM WHM-2-105-107 2019-07-18 22-30-19\WHM-2-105-1064.D Sample Name: WHM-2-106-RS

\begin{tabular}{|c|c|c|c|}
\hline Acq. Operator & : SYSTEM & Seq. Line & 5 \\
\hline Acq. Instrument & : 1260 & Location & 26 \\
\hline Injection Date & : 7/18/2019 11:47:07 PM & $\begin{array}{r}\text { Inj } \\
\text { Inj Volume }\end{array}$ & $\begin{array}{cc}: & 1 \\
: & 5.000\end{array}$ \\
\hline
\end{tabular}

Acq. Method : E: \DATA WHMIWHM-2-105-107 2019-07-18 22-30-19\AD-95-5-20min-5UL-1ML.M Last changed : 7/18/2019 10:30:19 PM by SYSTEM

Analysis Method: E: \DATA WHM\WHM-2-105-107 2019-07-18 22-30-19\AD-95-5-20min-5UL-1ML.M ( Sequence Method)

Last changed : 10/23/2019 9:58:28 AM by SYSTEM (modified after loading)

Additional Info: Peak(s) manually integrated

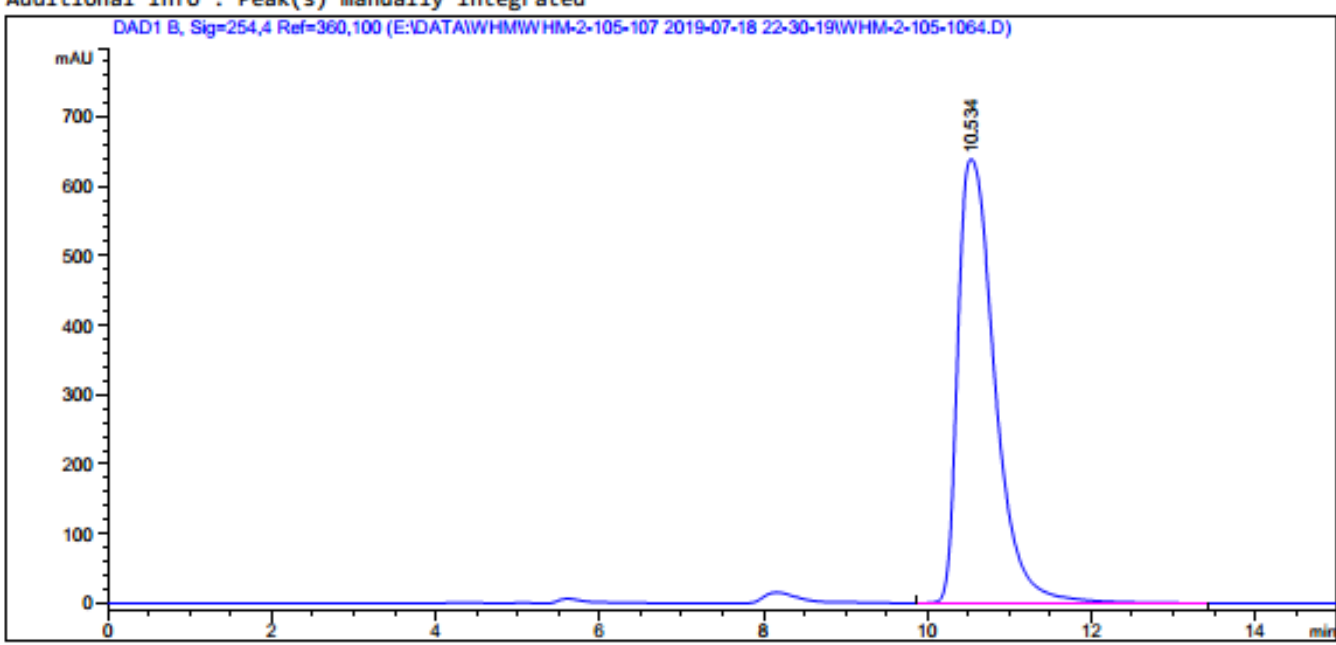

\begin{tabular}{|c|c|c|}
\hline Sorted By & : & Signal \\
\hline Multiplier & : & $1 . \theta 000$ \\
\hline Dilution & : & 1.0000 \\
\hline
\end{tabular}

Do not use Multiplier \& Dilution Factor with ISTDs

Signal 1: DAD1 B, Sig $=254,4$ Ref $=360,100$

\begin{tabular}{|c|c|c|c|c|}
\hline $\begin{array}{l}\text { Peak RetTime Type } \\
\# \text { [min] }\end{array}$ & $\begin{array}{l}\text { Width } \\
\text { [min] }\end{array}$ & $\begin{array}{c}\text { Area } \\
{[\mathrm{mAU} * \mathrm{~s}]}\end{array}$ & $\begin{array}{l}\text { Height } \\
\text { [mAU] }\end{array}$ & $\begin{array}{c}\text { Area } \\
\text { \% }\end{array}$ \\
\hline$-\cdots|-n|-|-|$ & & - & 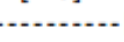 & - \\
\hline $110.534 \mathrm{BB}$ & 0.4813 & $1.99716 \mathrm{e} 4$ & 639.62867 & 100.0000 \\
\hline Totals : & & $1.99716 \mathrm{e} 4$ & 639.62067 & \\
\hline
\end{tabular}

*** End of Report *** 


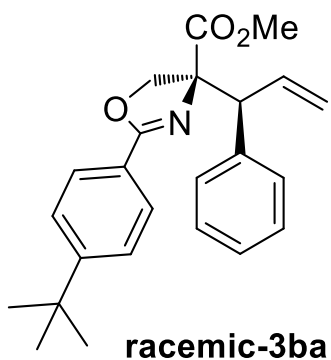

Data File E: IDATA WHM WWH-3-29-P-TBU-RAC 2019-89-17 16-19-28 WWH-3-29-P-TBU-Rac1.D Sample Name: WHM-3-29-P-TBU-RAC

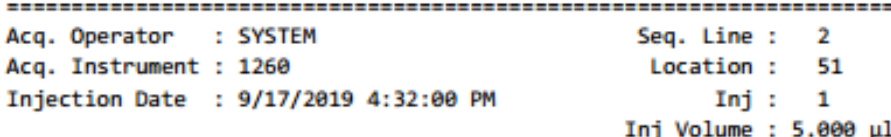

Acq. Method : E: \DATAWWHMWHM-3-29-P-TBU-Rac 2019-99-17 16-19-28\AD-95-5-20min-5UL-1ML.M Last changed : 9/17/2019 4:19:29 PM by SYSTEM

Analysis Method : E: \DATA WHM WHM-3-29-P-TBU-Rac 2019-09-17 16-19-28\AD-95-5-20min-5UL-1ML.M (Sequence Method)

Last changed : 10/23/2019 10:31:35 AM by SYSTEM

(modified after loading)

Additional Info: Peak(s) manually integrated

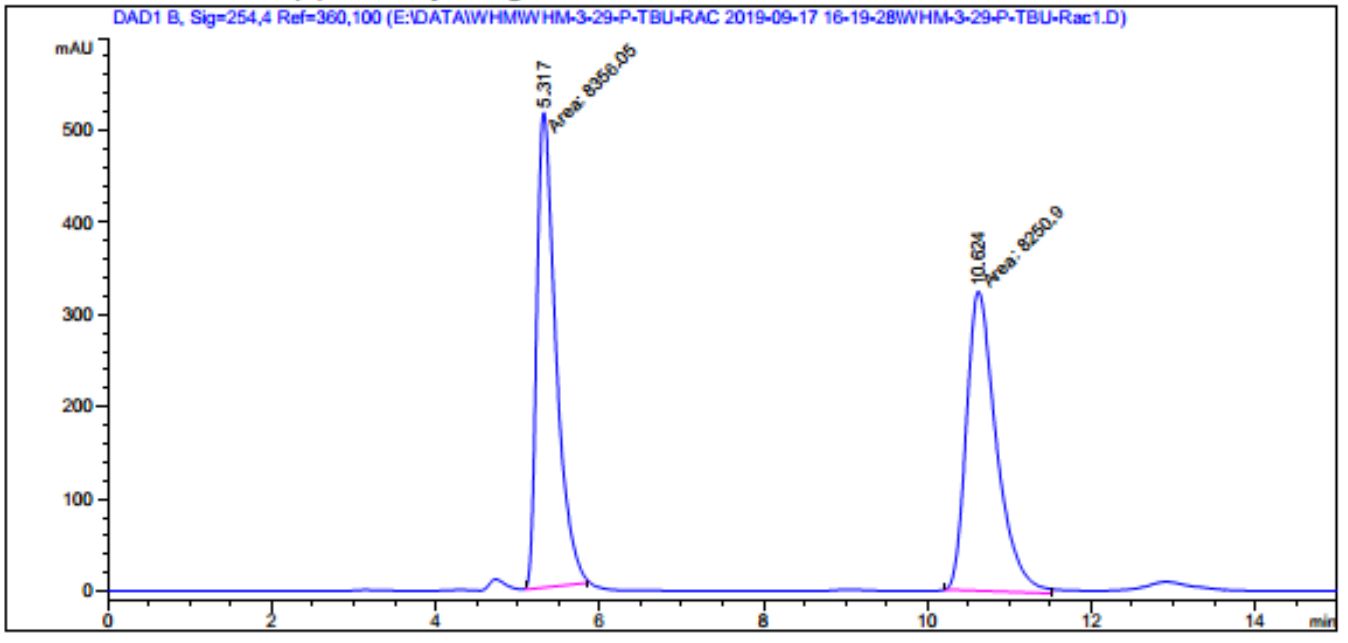

Area Percent Report

\begin{tabular}{|c|c|c|}
\hline Sorted By & : & Signal \\
\hline Multiplier & : & 1.0000 \\
\hline Dilution & : & 1.0000 \\
\hline
\end{tabular}

Do not use Multiplier \& Dilution Factor with ISTDs

Signal 1: DAD1 B, Sig $=254,4$ Ref $=360,100$

\begin{tabular}{|c|c|c|c|c|c|}
\hline $\begin{array}{c}\text { Peak } \\
\#\end{array}$ & $\begin{array}{l}\text { RetTime Type } \\
\text { [min] }\end{array}$ & $\begin{array}{l}\text { Width } \\
\text { [min] }\end{array}$ & $\begin{array}{c}\text { Area } \\
{\left[\mathrm{mAU}^{*} \mathrm{~s}\right]}\end{array}$ & $\begin{array}{l}\text { Height } \\
\text { [MAU] }\end{array}$ & $\begin{array}{c}\text { Area } \\
\%\end{array}$ \\
\hline$\ldots$ & $|-\ldots .0|-2$ & - & $|-\ldots+2|$ & |........... & $\ldots$ \\
\hline 1 & $5.317 \mathrm{MM}$ & 0.2704 & 8356.05273 & 514.97034 & 50.3166 \\
\hline 2 & $10.624 \mathrm{MM}$ & 0.4240 & 8250.90137 & 324.36035 & 49.6834 \\
\hline Total & 1s: & & $1.6607 \mathrm{le} 4$ & 839.33069 & \\
\hline
\end{tabular}

*** End of Report *** 


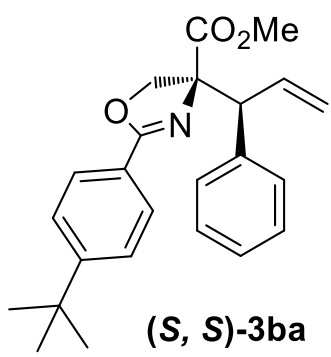

Data file E: \DATALWHM WHM-3-28-29 2019-09-09 18-23-00\WHM-3-28-293.D Sample Name: WHM-3-29-T-BU-RS
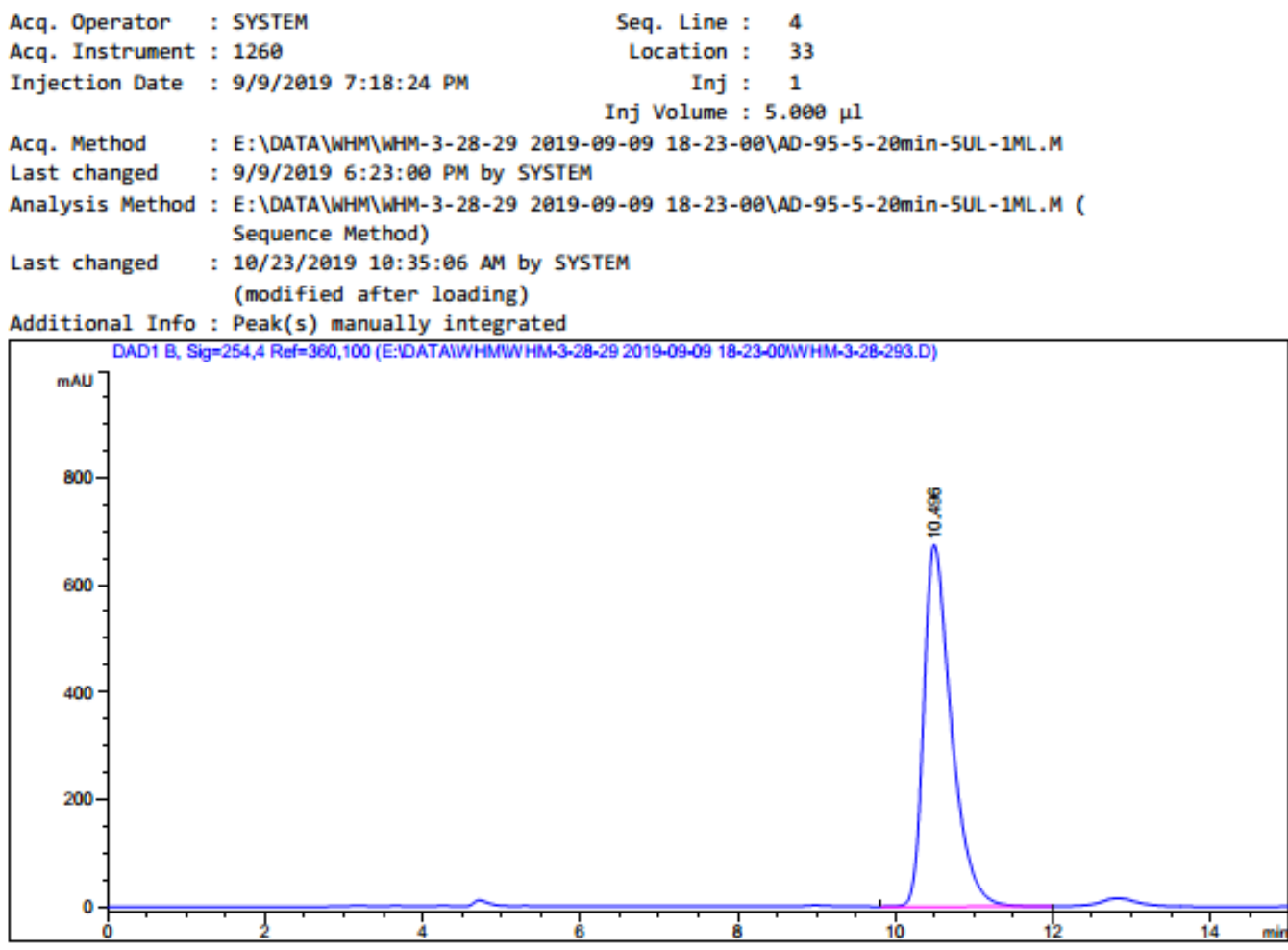

Area Percent Report

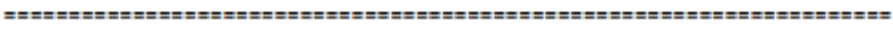

Sorted By : Signal

Multiplier : : 1.0000

Dilution : 1.0000

Do not use Multiplier \& Dilution Factor with ISTDs

Signal 1: DAD1 B, Sig $=254,4$ Ref $=360,100$

\begin{tabular}{|c|c|c|c|c|c|}
\hline $\begin{array}{c}\text { Peak } \\
\#\end{array}$ & $\begin{array}{l}\text { RetTime Type } \\
\text { [min] }\end{array}$ & $\begin{array}{l}\text { Width } \\
\text { [min] }\end{array}$ & $\begin{array}{c}\text { Area } \\
{\left[\mathrm{mAU}^{*} \mathrm{~s}\right]}\end{array}$ & $\begin{array}{l}\text { Height } \\
\text { [mAU] }\end{array}$ & $\begin{array}{c}\text { Area } \\
\text { \% }\end{array}$ \\
\hline
\end{tabular}

Totals : $\quad 1.64148 \mathrm{e} 4 \quad 674.85120$

*** End of Report *** 


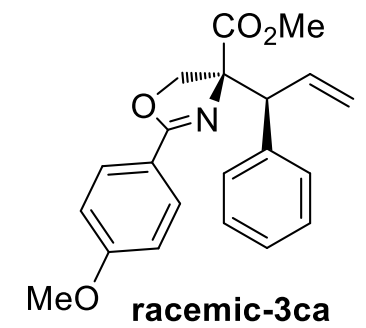

Data file E: DDATA WWHYWHM-3-38--37-RAC-T-BU- 2019-09-17 10-23-35 WWHM-3-38-37-RAC-P-TBU1.D Sample Name: WHM-3-37-P-OME-RAC

\begin{tabular}{|c|c|c|c|}
\hline Acq. Operator & : SYSTEM & Seq. Line : & 2 \\
\hline Acq. Instrument & : 1260 & Location : & 51 \\
\hline Injection Date & : 9/17/2019 10:36:04 AM & $\begin{array}{r}\text { Inj : } \\
\text { Inj Volume : }\end{array}$ & $\begin{array}{c}1 \\
2.00\end{array}$ \\
\hline
\end{tabular}

Acq. Method : E: \DATA WHMYWHM-3-38--37-RAC-T-BU- 2019-99-17 10-23-35 AD,95-5, 2UL, 1ML, 3EMIN.M

Last changed : 9/17/2019 10:23:35 AM by SYSTEM

Analysis Method : E: \DATA WHM WHM-3-38--37-RAC-T-BU- 2019-09-17 10-23-35 AD, 95-5, 2UL, 1ML, 3EMIN.M (Sequence Method)

Last changed : 10/23/2019 10:43:02 AM by SYSTEM (modified after loading)

Additional Info: Peak(s) manually integrated

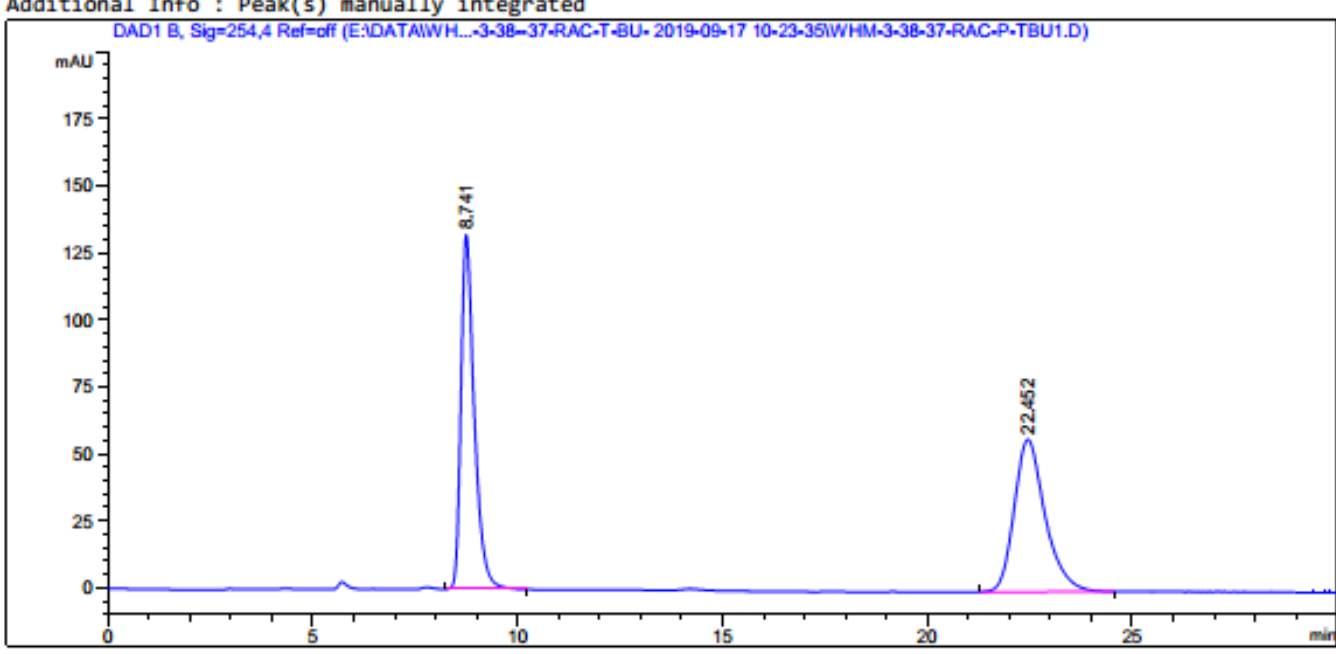

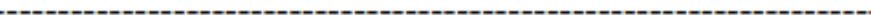

Area Percent Report

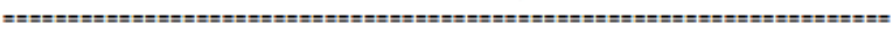

$\begin{array}{llc}\text { Sorted By } & : & \text { Signal } \\ \text { Multiplier } & : & 1.0000 \\ \text { Dilution } & : & 1.0000\end{array}$

Do not use Multiplier \& Dilution Factor with ISTDs

Signal 1: DAD1 B, Sig=254,4 Ref=off

\begin{tabular}{|c|c|c|c|c|c|}
\hline $\begin{array}{c}\text { Peak } \\
\#\end{array}$ & $\begin{array}{l}\text { RetTime Type } \\
\text { [min] }\end{array}$ & $\begin{array}{l}\text { Width } \\
\text { [min] }\end{array}$ & $\begin{array}{c}\text { Area } \\
{\left[\mathrm{mAU}^{*} \mathrm{~s}\right]}\end{array}$ & $\begin{array}{l}\text { Height } \\
\text { [mAU] }\end{array}$ & $\begin{array}{c}\text { Area } \\
\text { \% }\end{array}$ \\
\hline$\cdots$ & $--1-$ & & 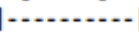 & - & - \\
\hline 1 & 8.741 BB & 0.3355 & 2991.36255 & 132.43779 & 50.0512 \\
\hline 2 & $22.452 \mathrm{BB}$ & 0.7232 & 2985.24756 & 56.88988 & 49.9488 \\
\hline Total & & & 5976.61011 & 189.32767 & \\
\hline
\end{tabular}

*** End of Report *** 


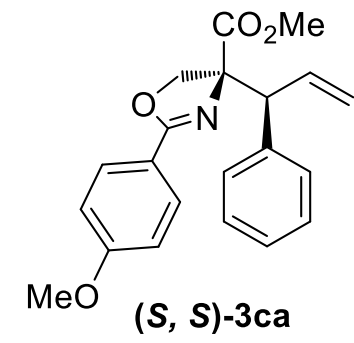

Data File E: \DATA WWH...M-3-37-P-OME-NAY-OMEY-2 2019-09-16 08-45-64\WHM-P-OME-NAY-OMEY-21.D Sample Name: WHM-3-37-P-OME-RS

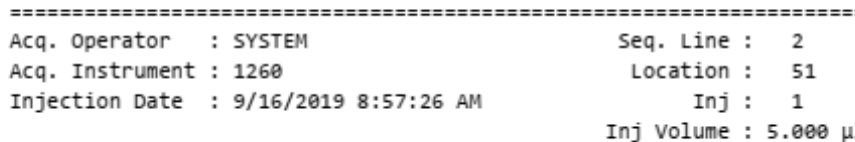

Acq. Method : E: \DATA WHHM WHM-3-37-P-OME-NAY-OMEY-2 2019-09-16 08-45-04\ADH-95-5-5UL35MIN.M

Last changed : 9/16/2019 8:45:04 AM by SYSTEM

Analysis Method: E: \DATA WHM WWHM-3-37-P-OME-NAY-OMEY-2 2019-09-16 08-45-04\ADH-95-5-5UL35MIN.M (Sequence Method)

Last changed : 10/23/2019 11:12:15 AM by SYSTEM

(modified after loading)

Additional Info: Peak(s) manually integrated

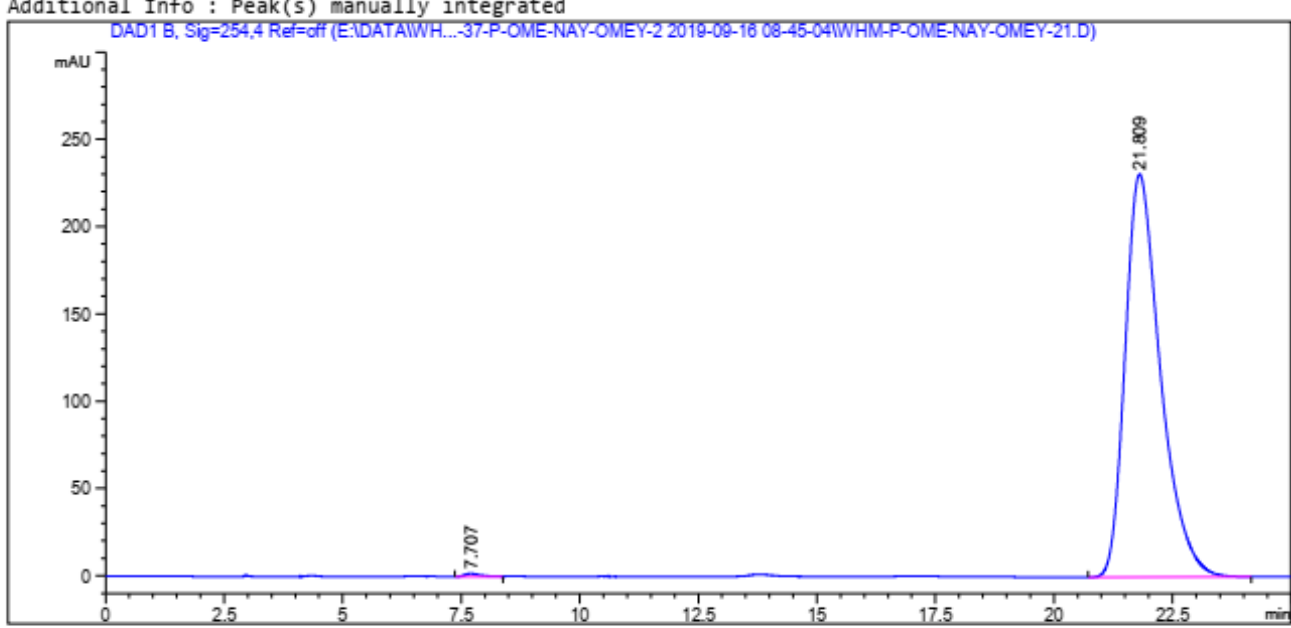

Area Percent Report

\begin{tabular}{|c|c|c|}
\hline Sorted By & : & signal \\
\hline Multiplier & : & 1.0000 \\
\hline Dilution & : & 1.0000 \\
\hline
\end{tabular}

Signal 1: DAD1 B, Sig $=254,4$ Ref $=0$ ff

\begin{tabular}{|c|c|c|c|c|c|}
\hline $\begin{array}{c}\text { Peak } \\
\#\end{array}$ & $\begin{array}{l}\text { RetTime Type } \\
\text { [min] }\end{array}$ & $\begin{array}{l}\text { Width } \\
\text { [min] }\end{array}$ & $\begin{array}{c}\text { Area } \\
{[\mathrm{mAU} * \mathrm{~s}]}\end{array}$ & $\begin{array}{l}\text { Height } \\
\text { [mAU] }\end{array}$ & $\begin{array}{c}\text { Area } \\
\%\end{array}$ \\
\hline$\cdots$ & -... & --1 & | & | & | \\
\hline 1 & $7.707 \mathrm{BB}$ & 0.2670 & 38.43193 & 1.75809 & 0.3198 \\
\hline 2 & $21.809 \mathrm{BB}$ & 0.7525 & $1.19777 \mathrm{e} 4$ & 230.80034 & 99.6802 \\
\hline
\end{tabular}

$1.20162 \mathrm{e} 4 \quad 232.55843$

$* * *$ End of Report $* * *$ 
<smiles>C=CC(c1ccccc1)C(C)(C)C(C)=NC(=O)c1cc(OC)c(OC)c(OC)c1</smiles>

\section{racemic-3da}

Data file E: \DATAWWHMWHM-3-28-29-32RAC 2019-e9-12 17-67-44\whm-3-28-29-321.D Sample Name: WHM-3-28-3-OME-RAC

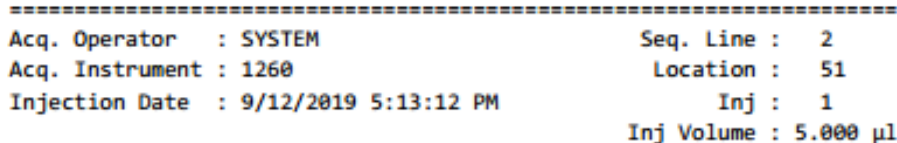

Acq. Method : E: \DATA :WHMYWHM-3-28-29-32RAC 2019-99-12 17-07-44\AD-95-5-28min-5UL-1ML.M Last changed : 9/12/2019 5:07:45 PM by SYSTEM

Analysis Method: E: \DATA WWHMWHM-3-28-29-32RAC 2019-09-12 17-07-44\AD-95-5-28min-5UL-1ML.M ( Sequence Method)

Last changed : 10/23/2019 $7: 14: 28$ PM by SYSTEM

(modified after loading)

Additional Info : Peak(s) manually integrated

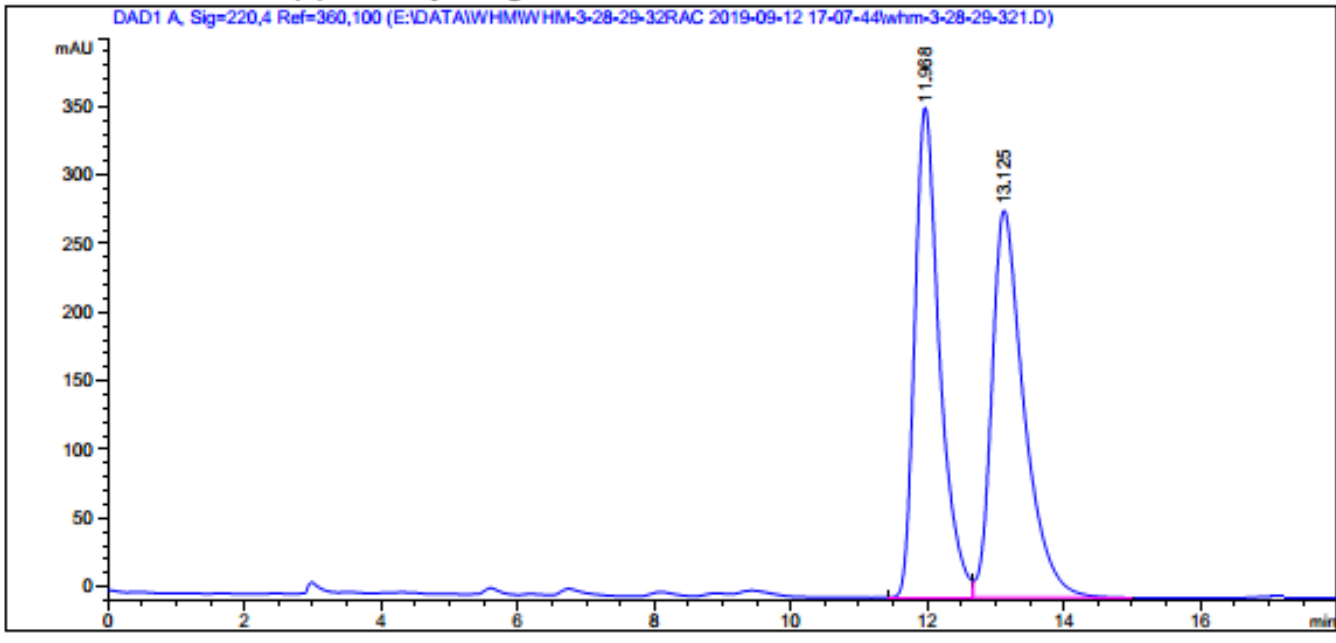

Area Percent Report

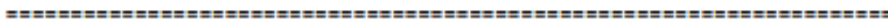

Sorted By : : Signal

Multiplier $\quad: \quad 1.0000$

Dilution : 1.0000

Do not use Multiplier \& Dilution Factor with ISTDs

Signal 1: DAD1 A, Sig=220,4 Ref $=360,100$

\begin{tabular}{|c|c|c|c|c|c|}
\hline $\begin{array}{c}\text { Peak } \\
\#\end{array}$ & $\begin{array}{l}\text { RetTime Type } \\
\text { [min] }\end{array}$ & $\begin{array}{l}\text { Width } \\
\text { [min] }\end{array}$ & $\begin{array}{c}\text { Area } \\
{\left[\mathrm{mAU}^{*} \mathrm{~s}\right]}\end{array}$ & $\begin{array}{l}\text { Height } \\
\text { [mAU] }\end{array}$ & $\begin{array}{l}\text { Area } \\
\text { \% }\end{array}$ \\
\hline-1 & 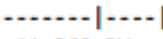 & & . & . & 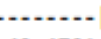 \\
\hline 1 & $11.968 \mathrm{BV}$ & 0.3784 & 9939.32617 & 357.01718 & 49.4791 \\
\hline 2 & $13.125 \mathrm{VB}$ & 0.4887 & 9229.64063 & 282.10349 & 50.5209 \\
\hline Tot: & & & $1.82690 \mathrm{e} 4$ & 639.12067 & \\
\hline
\end{tabular}

*** End of Report *** 
<smiles>C=CC(c1ccccc1)C(C)(COC)N=C(C)c1cc(OC)c(OC)c(OC)c1</smiles>

\section{$(S, S)-3 d a$}

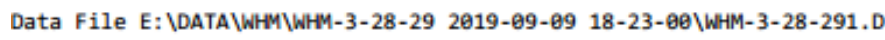
Sample Name: WHM-3-28-3-OME-RS
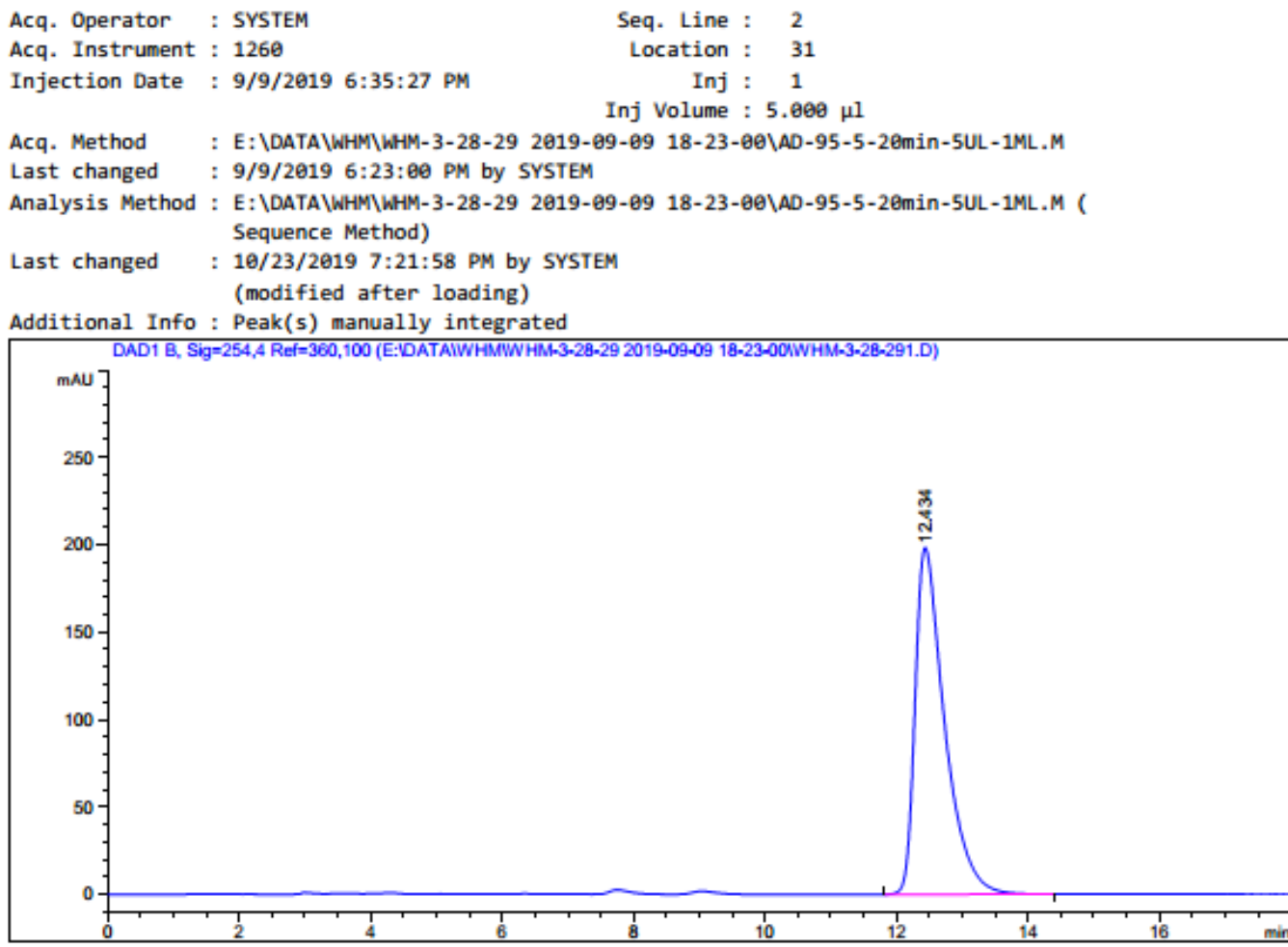

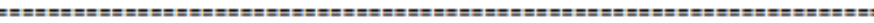

Area Percent Report

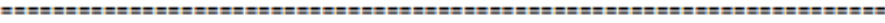

Sorted By : : Signal

Multiplier $\quad: \quad 1.0000$

Dilution : 1.0000

Do not use Multiplier \& Dilution Factor with ISTDs

Signal 1: DAD1 B, Sig $=254,4$ Ref $=360,100$

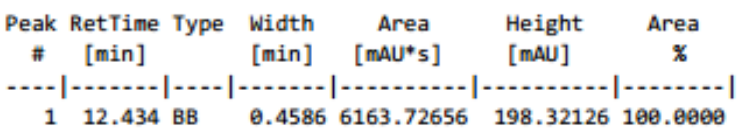

Totals:

$6163.72656 \quad 198.32126$

*** End of Report *** 


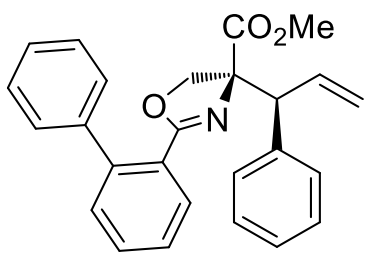

racemic-3ea

Data file E: \DATA \WHИ\WHM-3-28-29-32RAC 2019-99-12 17-67-44\whm-3-28-29-325.D Sample Name: WHM-3-32-BP-RAC
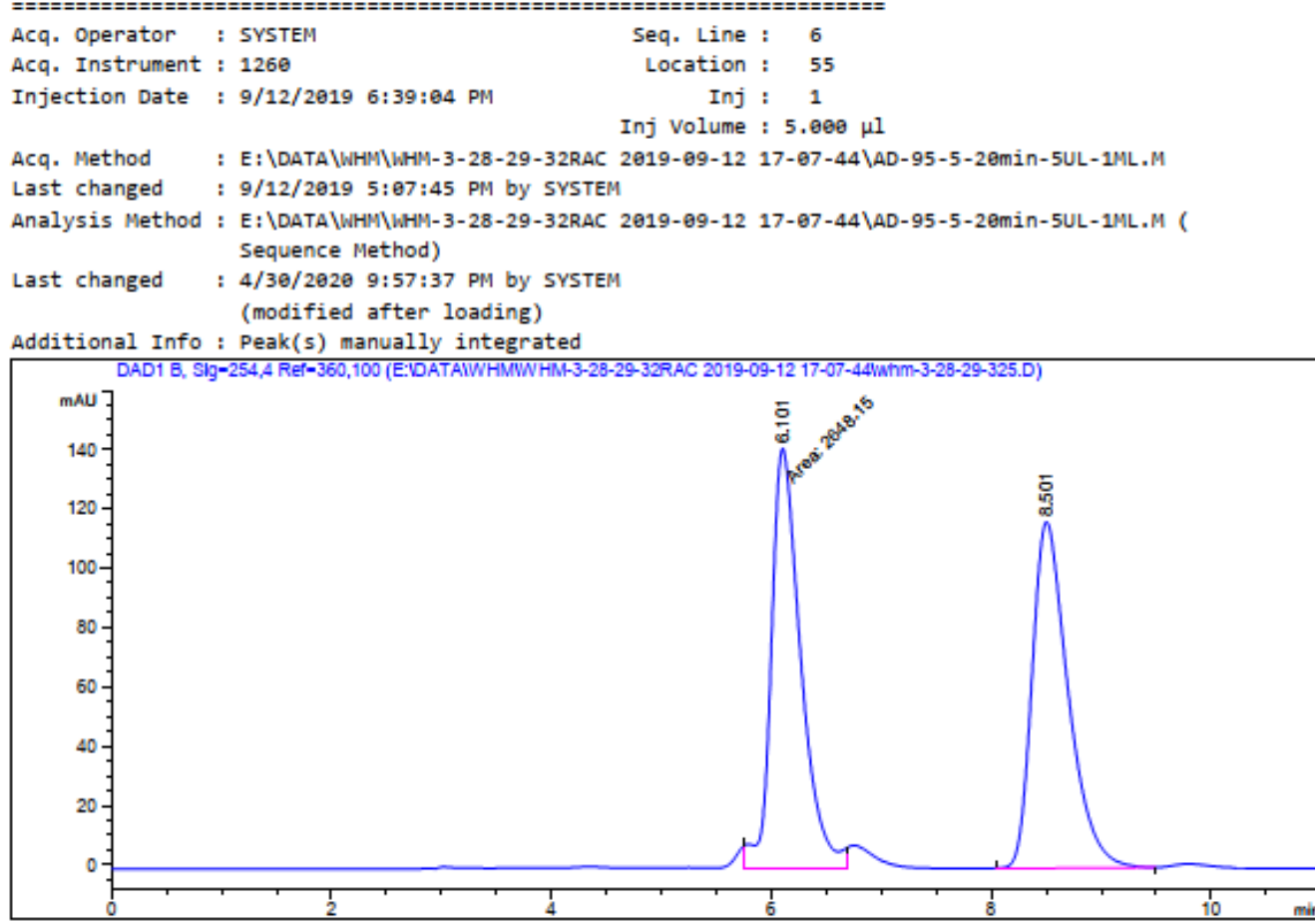

\section{Area Percent Report}

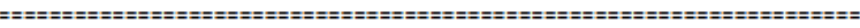

$\begin{array}{lll}\text { Sorted By } & : & \text { Signal } \\ \text { Multiplier } & : & 1.0000 \\ \text { Dilution } & : & 1.0000\end{array}$

Do not use Multiplier \& Dilution Factor with ISTDS

Signal 1: DAD1 B, Sig $=254,4$ Ref $=360,100$

\begin{tabular}{cccccc}
$\begin{array}{c}\text { Peak RetTime Type } \\
\text { \# } \\
\text { [min] }\end{array}$ & $\begin{array}{c}\text { Width } \\
\text { [min] }\end{array}$ & $\begin{array}{c}\text { Area } \\
\text { [mAU+s] }\end{array}$ & $\begin{array}{c}\text { Height } \\
\text { [mAU] }\end{array}$ & $\begin{array}{c}\text { Area } \\
\%\end{array}$ \\
\hdashline 1 & 6.101 MF & 0.3123 & 2648.15479 & 141.33090 & 49.9545 \\
2 & 8.501 BB & 0.3415 & 2652.98193 & 116.56572 & 50.6455
\end{tabular}

Totals :

$5301.13672 \quad 257.89662$

$+*+$ End of Report *** 


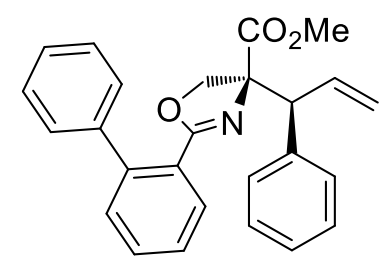

$(S, s)$-3ea

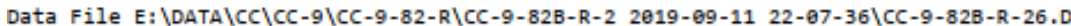
Sample Name: WHM-3-32-BP-RS

\begin{tabular}{|c|c|c|c|}
\hline Acq. Operator & : SYSTEM & Seq. Line & 7 \\
\hline Injection Date & : 9/11/2019 11:52:21 PM & Inj & 1 \\
\hline
\end{tabular}

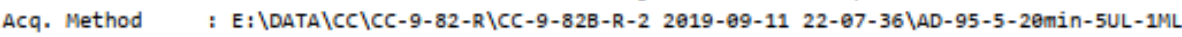
. $\mathrm{M}$

Last changed : 9/11/2019 10:18:22 PM by SYSTEM

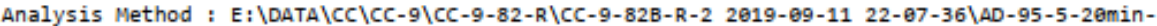
5UL-1ML.M (Sequence Method)

Last changed : 4/30/2020 9:41:28 PM by SYSTEM (modified after loading)

Additional Info: Peak(s) manually integrated

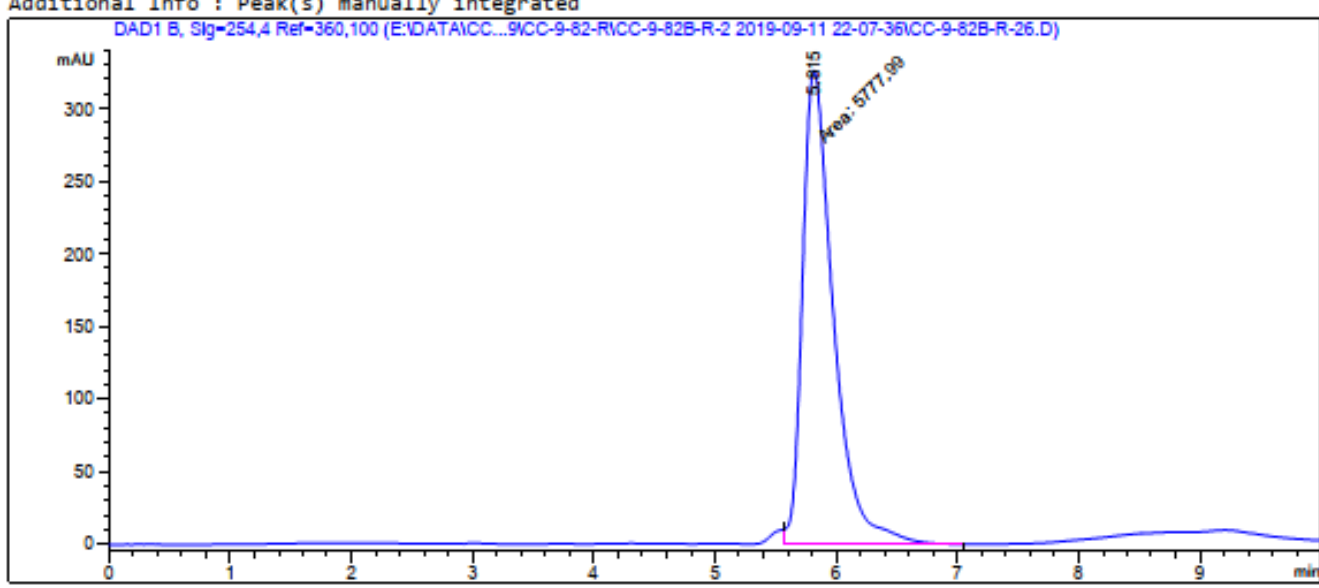

Area Percent Report

\begin{tabular}{|c|c|c|}
\hline Sorted By & : & Signal \\
\hline Multiplier & : & 1.0000 \\
\hline Dilution & : & 1.0000 \\
\hline
\end{tabular}

Do not use Multiplier \& Dilution Factor with ISTDs

Signal 1: DAD1 B, Sig $=254,4$ Ref $=360,100$

\begin{tabular}{|c|c|c|c|c|c|}
\hline $\begin{array}{c}\text { Peak } \\
\#\end{array}$ & $\begin{array}{l}\text { RetTime Type } \\
\text { [min] }\end{array}$ & $\begin{array}{l}\text { Width } \\
\text { [min] }\end{array}$ & $\begin{array}{c}\text { Area } \\
{\left[\mathrm{maU}^{+} \mathrm{s}\right]}\end{array}$ & $\begin{array}{l}\text { Height } \\
\text { [mAU] }\end{array}$ & $\begin{array}{c}\text { Area } \\
\%\end{array}$ \\
\hline$\cdots$ & $|\cdots|-\mid$ & 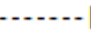 & | & | & $|+\ldots . . .6|$ \\
\hline 1 & $5.815 \mathrm{FM}$ & 0.2954 & 5777.98975 & 326.01859 & 100.0000 \\
\hline Total & Is : & & 5777.98975 & 326.01859 & \\
\hline
\end{tabular}

*** End of Report *** 


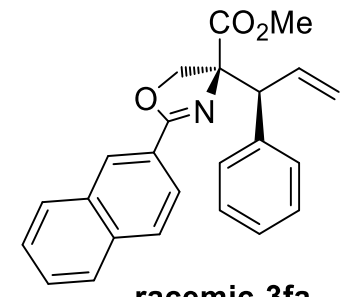

racemic-3fa

Data file E: \DATA WHM WWHM-2-133-2-NA-RAC 2019-10-28 21-45-38\WHM-2-NA-1331.D Sample Name: WHM-2-133-2-NA-RAC

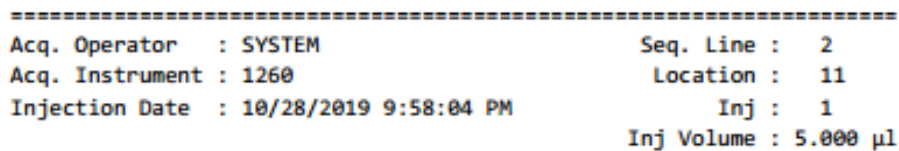

Acq. Method : E: \DATA WWMYWHM-2-133-2-NA-RAC 2019-10-28 21-45-38\AD-95-5-20min-5UL-1ML.M Last changed : 10/28/2019 9:45:38 PM by SYSTEM

Analysis Method: E: \DATA WHMYWHM-2-133-2-NA-RAC 2019-10-28 21-45-38\AD-95-5-20min-5UL-1ML.M (Sequence Method)

Last changed : 11/17/2019 11:50:06 AM by SYSTEM (modified after loading)

Additional Info: Peak(s) manually integrated
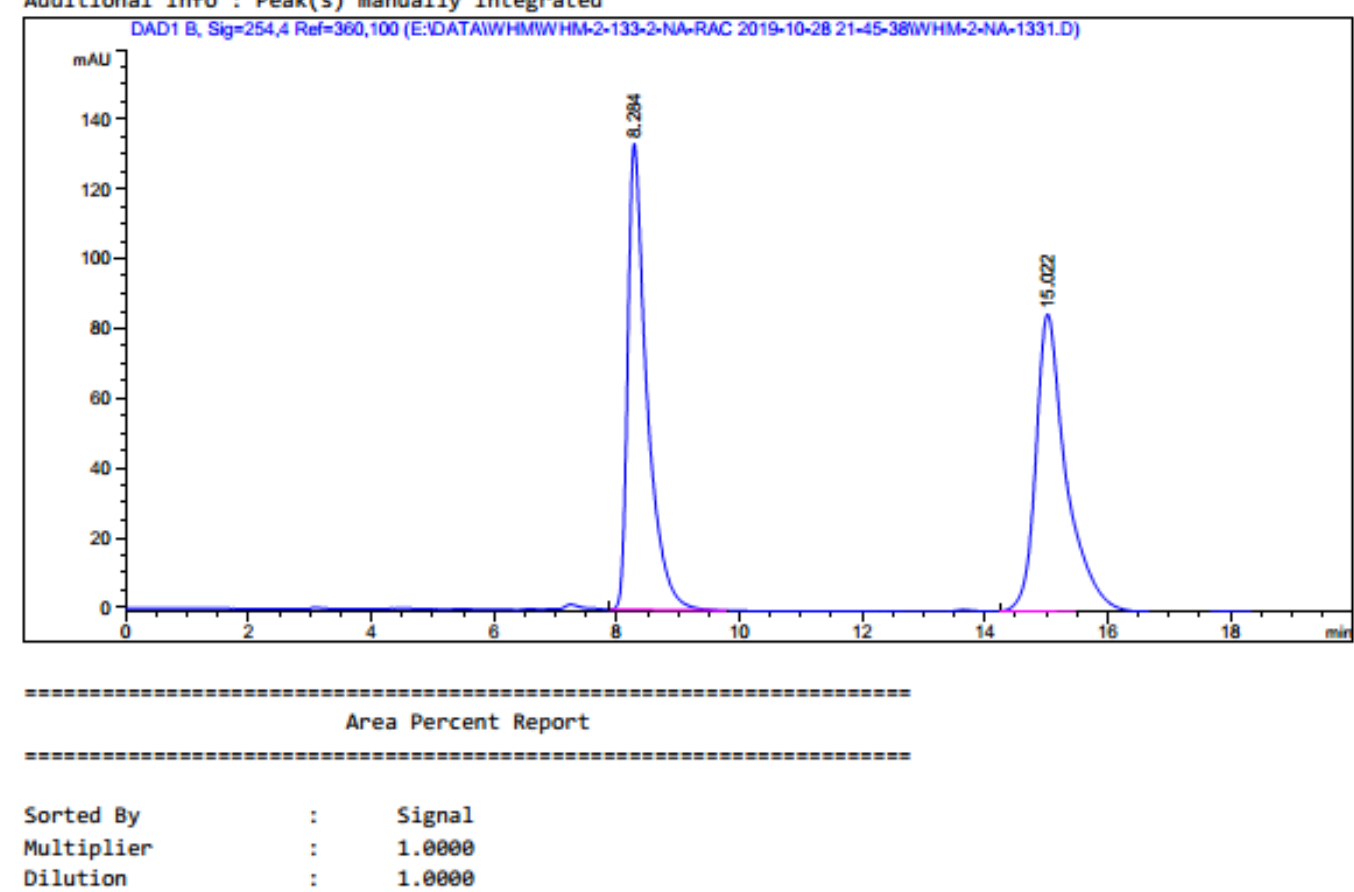

Do not use Multiplier \& Dilution Factor with ISTDs

Signal 1: DAD1 B, Sig $=254,4$ Ref $=360,100$

\begin{tabular}{|c|c|c|c|c|c|}
\hline $\begin{array}{c}\text { Peak } \\
\#\end{array}$ & $\begin{array}{l}\text { RetTime Type } \\
\text { [min] }\end{array}$ & $\begin{array}{l}\text { Width } \\
\text { [min] }\end{array}$ & $\begin{array}{c}\text { Area } \\
{\left[\mathrm{mAU}^{*} \mathrm{~s}\right]}\end{array}$ & $\begin{array}{l}\text { Height } \\
\text { [mAU] }\end{array}$ & $\begin{array}{c}\text { Area } \\
\text { \% }\end{array}$ \\
\hline 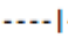 & $|-\ldots+|$ & & | & | & $\mid$ \\
\hline 1 & 8.284 BB & 0.3130 & 2884.40698 & 133.55588 & 49.6397 \\
\hline 2 & $15.022 \mathrm{BB}$ & 0.4989 & 2926.27954 & 85.15077 & 50.3603 \\
\hline נבד & & & 5810.68652 & 218.70665 & \\
\hline
\end{tabular}

*** End of Report *** 


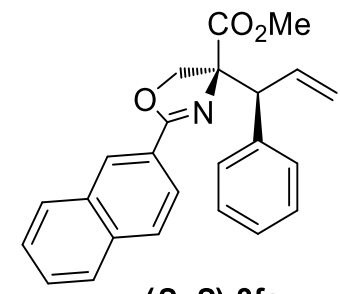

$(S, S)-3 f a$

Data File E: \DATA WHM WHM-2-109-124-133-3-13 2019-10-27 21-03-37\WHM-2-109-124-133-3-133.D Sample Name: WHM-2-133-2-NA-RS-2

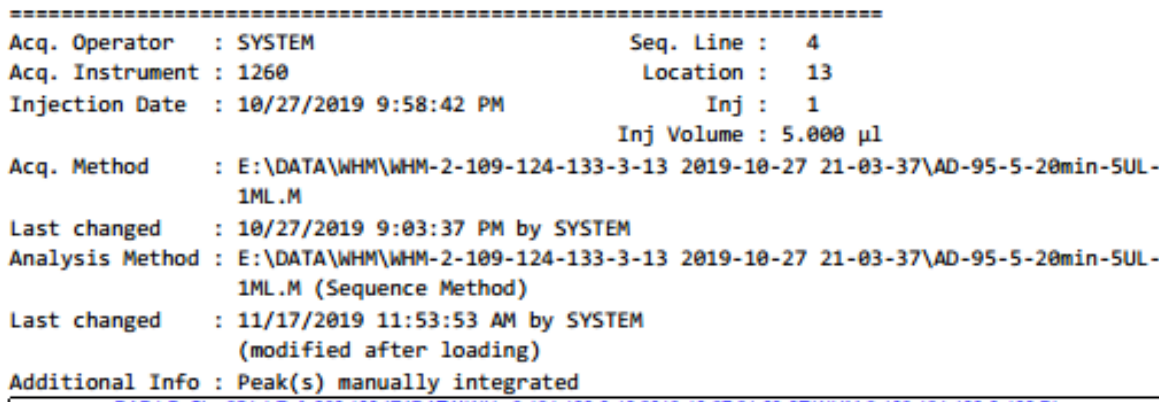

Acq. Method : E: \DATA WWM WHM-2-109-124-133-3-13 2019-10-27 21-03-37 $A D-95-5-20 m i n-5 U L-$ $1 \mathrm{ML}, \mathrm{M}$

Last changed : 10/27/2019 9:03:37 PM by SYSTEM

Analysis Method : E: \DATA\WHM\WHM-2-109-124-133-3-13 2019-10-27 21-63-37\AD-95-5-20min-5UL1ML.M (Sequence Method)

Last changed : 11/17/2019 11:53:53 AM by SYSTEM (modified after loading)

Additional Info : Peak(s) manually integrated

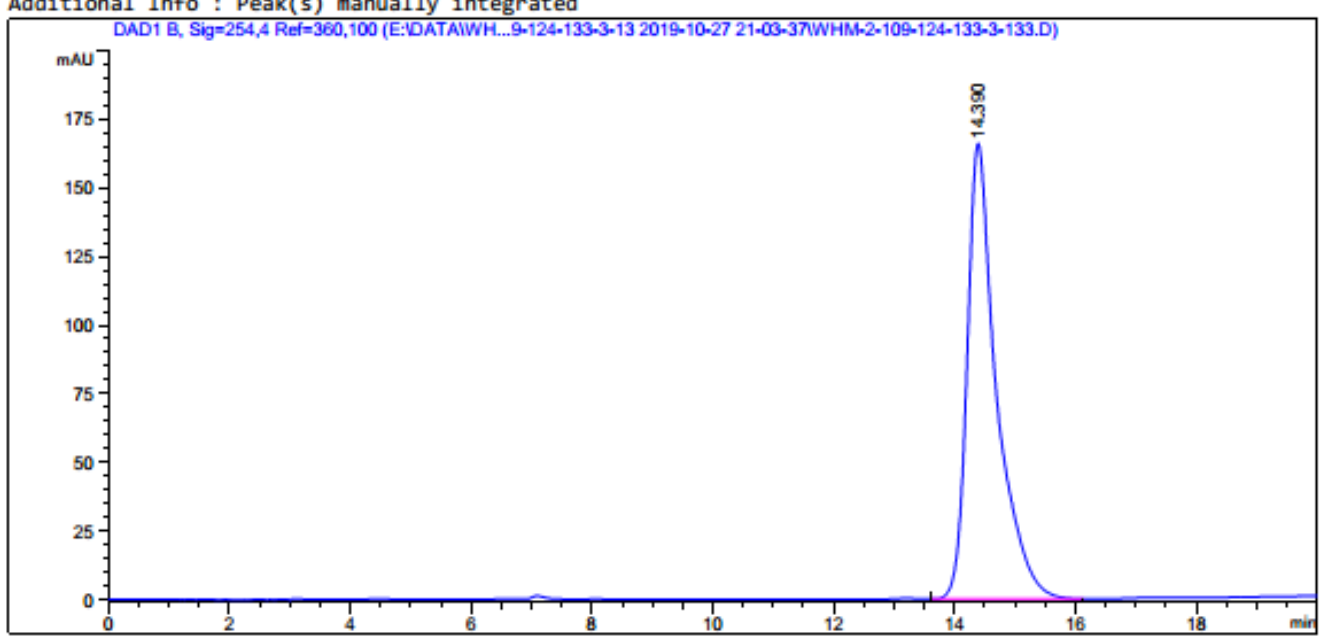

Area Percent Report

\begin{tabular}{|c|c|}
\hline Sorted By & Signal \\
\hline Multiplier & 1.0000 \\
\hline Dilution & $1 . \theta 000$ \\
\hline
\end{tabular}

Do not use Multiplier \& Dilution Factor with ISTDs

Signal 1: DAD1 B, Sig $=254,4$ Ref $=360,100$

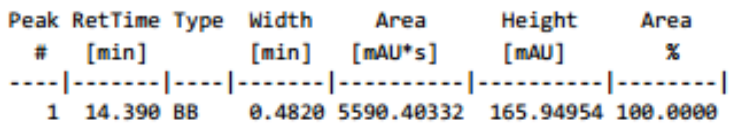

Totals :

$5590.40332 \quad 165.94954$ 


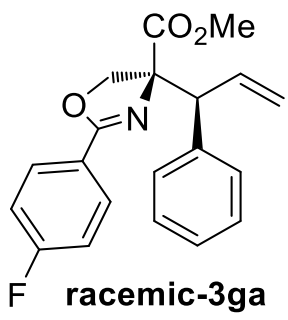

Data file E: \DATAWWHM WHM-3-38--37-RAC-T-BU- 2019-09-17 10-23-35 \WHM-3-38-37-RAC-P-TBU2.D Sample Name: WHM-3-38-P-F-RAC

\begin{tabular}{|c|c|c|c|}
\hline Acq. Operator & : SYSTEM & Seq. Line : & 3 \\
\hline Acq. Instrument & : 1260 & Location : & 52 \\
\hline Injection Date & : 9/17/2019 11:07:34 AM & $\begin{array}{r}\text { Inj : } \\
\text { Inj Volume : }\end{array}$ & $\begin{array}{c}1 \\
: 5.000 \mu 1\end{array}$ \\
\hline
\end{tabular}

Acq. Method : E: \DATA WWHMWHM-3-38--37-RAC-T-BU- 2019-99-17 10-23-35\AD-95-5-20min-5UL1ML.M

Last changed : 9/17/2019 10:23:35 AM by SYSTEM

Analysis Method: E: \DATA \WHM\WHM-3-38--37-RAC-T-BU- 2019-99-17 10-23-35\AD-95-5-20min-5UL1 ML.M (Sequence Method)

Last changed : 10/23/2019 11:22:56 AM by SYSTEM (modified after loading)

Additional Info: Peak(s) manually integrated

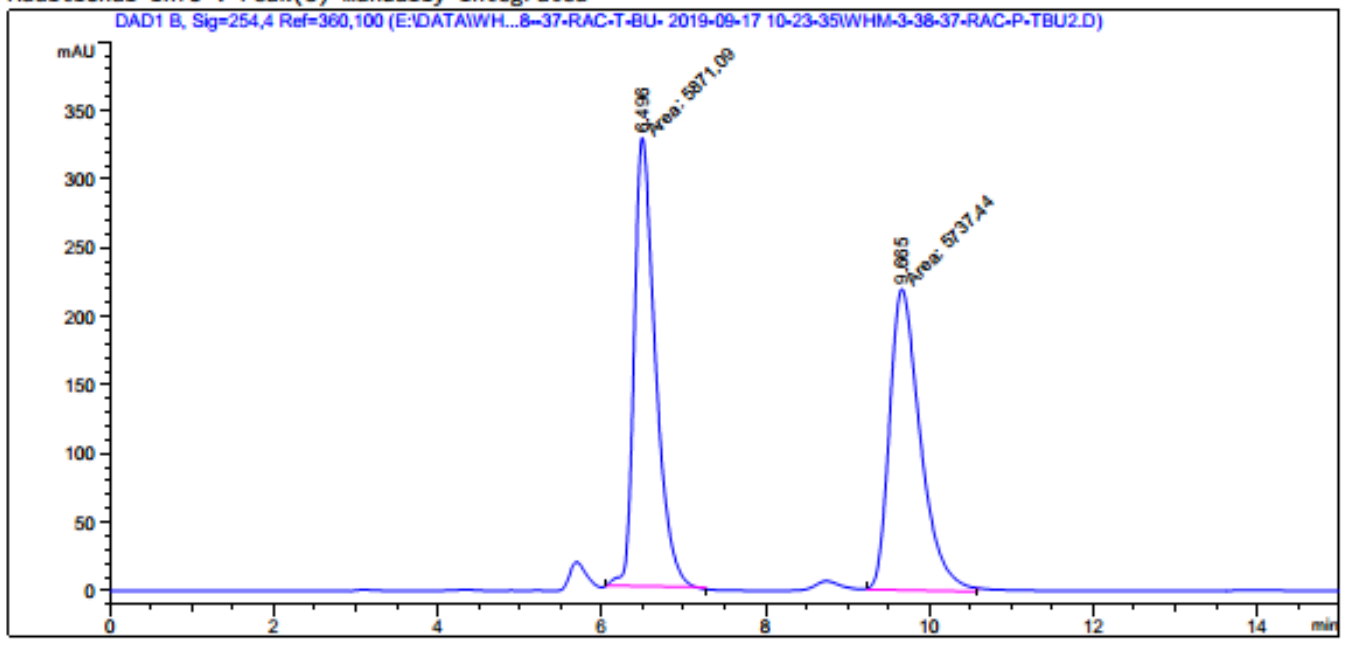

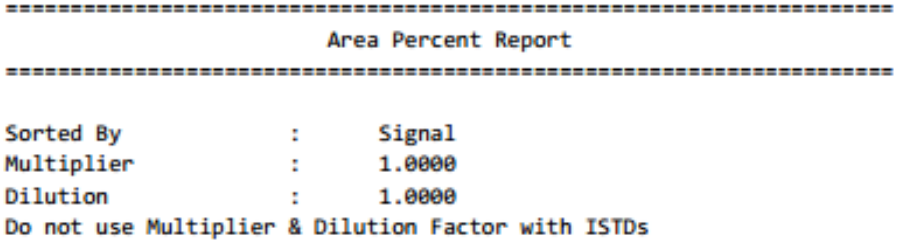

Signal 1: DAD1 B, Sig $=254,4$ Ref $=360,100$

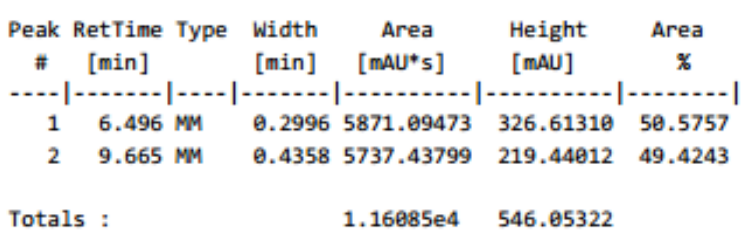

*** End of Report *** 
<smiles></smiles>

\section{$(S, S)$-3ga}

Data File E: \DATA WHM WHM-3-38-P-F 2019-09-16 20-39-36\WHM-3-381.D Sample Name: WHM-3-38-P-F-RS

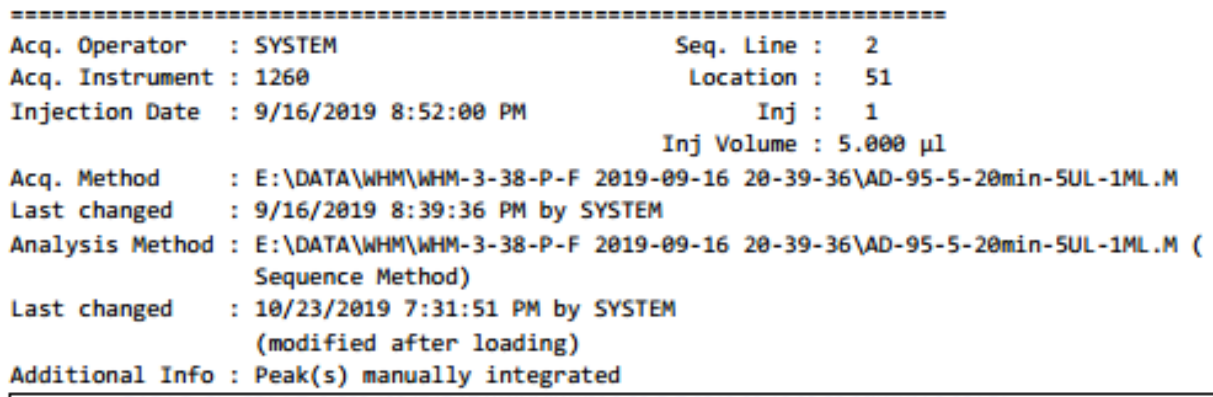

Acq. Method : E: \DATA WHMYWHM-3-38-P-F 2019-69-16 20-39-36\AD-95-5-20min-5UL-1ML.M Last changed : 9/16/2019 8:39:36 PM by SYSTEM

Analysis Method : E: \DATA WHMYWHM-3-38-P-F 2019-09-16 20-39-36\AD-95-5-20min-5UL-1ML.M ( Sequence Method)

Last changed : 10/23/2019 7:31:51 PM by SYSTEM (modified after loading)

Additional Info : Peak(s) manually integrated

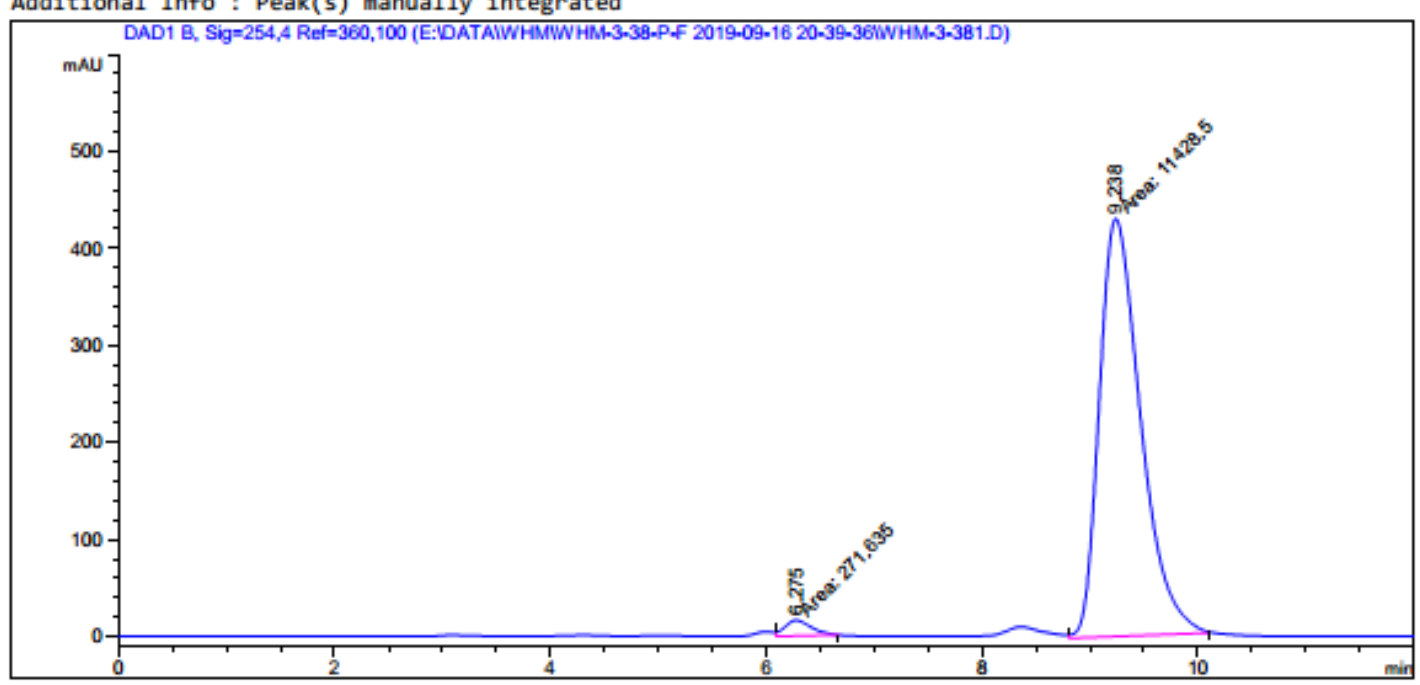

Area Percent Report

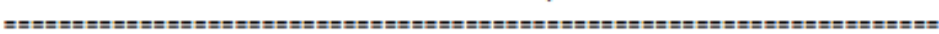

$\begin{array}{lll}\text { Sorted By } & : & \text { Signal } \\ \text { Multiplier } & : & 1.0000 \\ \text { Dilution } & : & 1.0000\end{array}$

Do not use Multiplier \& Dilution Factor with ISTDs

Signal 1: DAD1 B, Sig=254,4 Ref $=360,100$

\begin{tabular}{|c|c|c|c|c|c|}
\hline $\begin{array}{c}\text { Peak } \\
\#\end{array}$ & $\begin{array}{l}\text { RetTime Type } \\
\text { [min] }\end{array}$ & $\begin{array}{l}\text { Width } \\
\text { [min] }\end{array}$ & $\begin{array}{c}\text { Area } \\
{\left[\mathrm{mAU}^{*} \mathrm{~s}\right]}\end{array}$ & $\begin{array}{l}\text { Height } \\
\text { [mAU] }\end{array}$ & $\begin{array}{c}\text { Area } \\
\text { \% }\end{array}$ \\
\hline$\cdots$ & 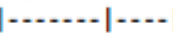 & - & - & 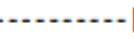 & 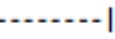 \\
\hline 1 & $6.275 \mathrm{MM}$ & 0.2830 & 271.63516 & 15.99559 & 2.3216 \\
\hline 2 & $9.238 \mathrm{MM}$ & 0.4425 & $1.14285 \mathrm{e} 4$ & 430.49796 & 97.6784 \\
\hline Tot & & & $1.17802 \mathrm{e} 4$ & 446.49354 & \\
\hline
\end{tabular}

*** End of Report *** 


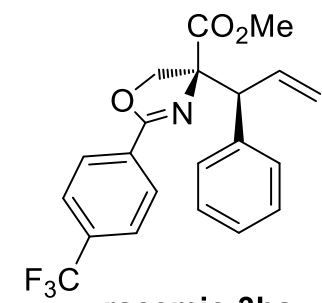

racemic-3ha

Data file E: \DATA WHM SXXS-8-131-OP 2019-09-01 13-51-64\WHM-3-13-3-15.D Sample Name: WHM-3-13-P-CF3-RAC

\begin{tabular}{|c|c|c|c|}
\hline $\begin{array}{l}\text { Acq. Operator } \\
\text { Acq. Instrument }\end{array}$ & $\begin{array}{l}: \text { SYSTEM } \\
: 1260\end{array}$ & $\begin{array}{l}\text { Seq. Line : } \\
\text { Location : }\end{array}$ & $\begin{array}{ll}: & 1 \\
: & 61\end{array}$ \\
\hline Injection Date & : 9/1/2019 1:52:27 PM & $\begin{array}{r}\text { Inj : } \\
\text { Inj Volume : }\end{array}$ & $\begin{array}{l}:=1 \\
: 3.000\end{array}$ \\
\hline
\end{tabular}

Acq. Method : E: \DATAWWHMSXXS-8-131-OP 2019-09-01 13-51-04\IDH-95-5--3UL-40MIN1. AML.M

Last changed : 9/1/2019 2:24:35 PM by SYSTEM

(modified after loading)

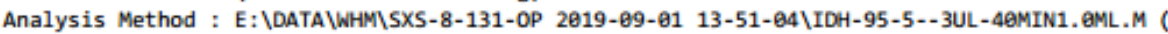
Sequence Method)

Last changed : 10/23/2019 10:02:14 AM by SYSTEM (modified after loading)

Additional Info: Peak(s) manually integrated

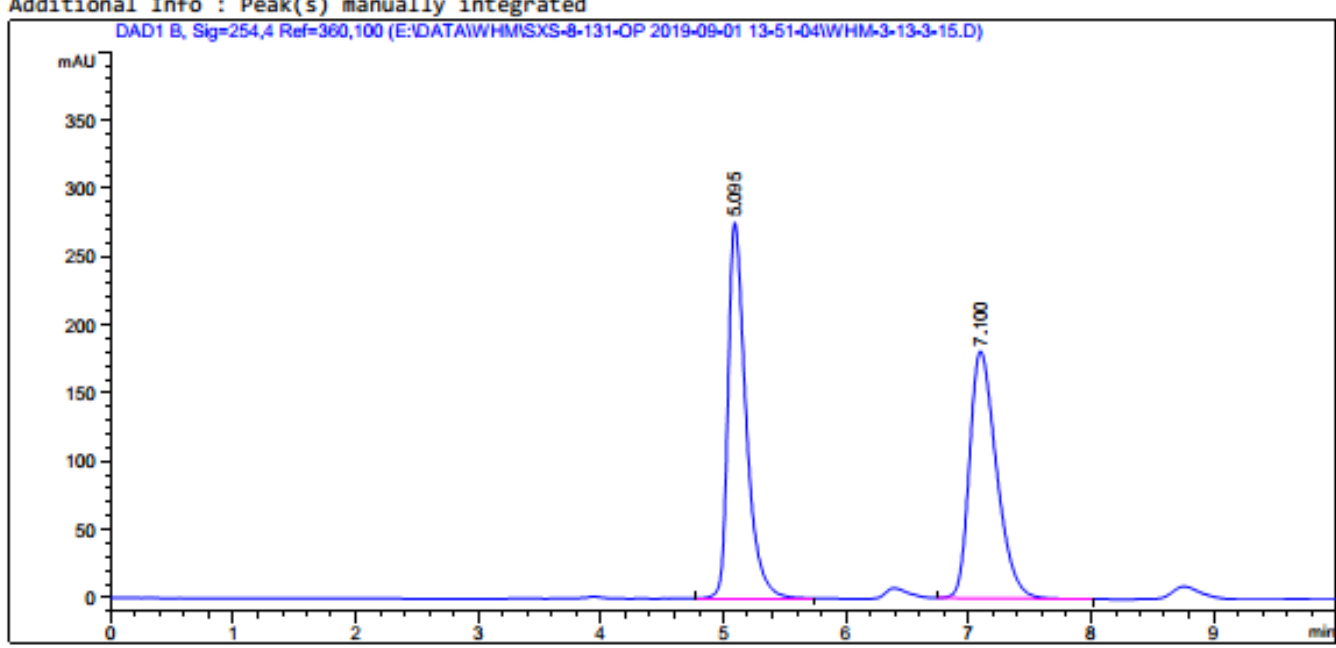

Area Percent Report

\begin{tabular}{|c|c|c|}
\hline Sorted By & : & Signal \\
\hline Multiplier & : & 1.0000 \\
\hline Dilution & : & $1 . \theta 000$ \\
\hline
\end{tabular}

Signal 1: DAD1 B, Sig $=254,4$ Ref $=360,100$

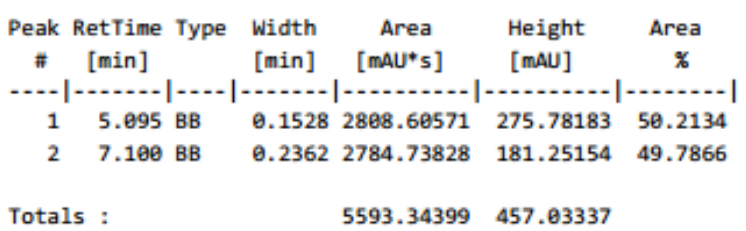

*** End of Report *** 
<smiles>C=CC(c1ccccc1)C(CO)(COCc1ccc(C(F)(F)F)cc1)C(C)=O</smiles>

$(S, S)-3$ ha

Data file E: \DATA WHM WHM-3-13-15-P-CF3-2 2019-09-08 99-15-57\WHM-3-13-15-21.D Sample Name: WHM-3-13-P-CF3-RS-2

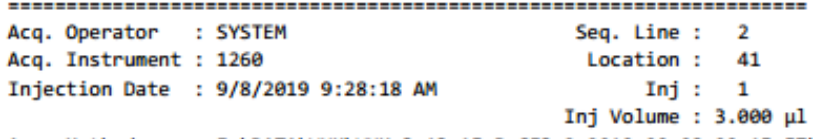

Acq. Method : E: \DATA WHMIWHM-3-13-15-P-CF3-2 2019-09-08 99-15-57\IDH-95-5--3UL-15MIN1.

Last changed : 9/8/2019 9:15:58 AM by SYSTEM

Analysis Method: E: (DATA WHMIWHM-3-13-15-P-CF3-2 2019-09-08 09-15-57\IDH-95-5--3UL-15MIN1. OML.M (Sequence Method)

Last changed : 10/23/2019 10:17:43 AM by SYSTEM

(modified after loading)

Additional Info: Peak(s) manually integrated

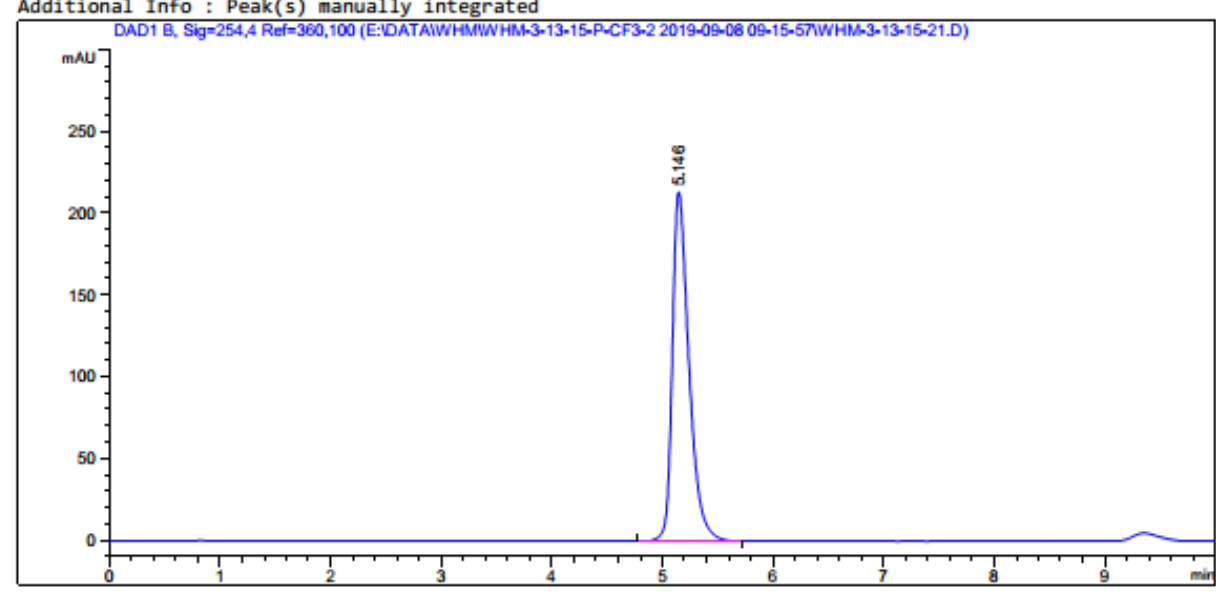

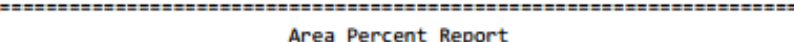

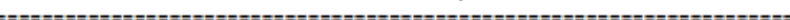

$\begin{array}{lll}\text { Sorted By } & : & \text { Signal } \\ \text { Multiplier } & : & 1.0000 \\ \text { Dilution } & : & 1.0000\end{array}$

Do not use Multiplier \& Dilution Factor with ISTD

Signal 1: DAD1 B, Sig $=254,4$ Ref $=360,100$

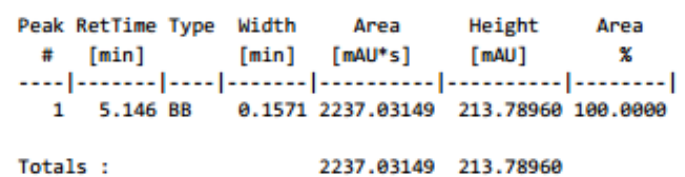

*** End of Report *** 
<smiles>C=C[C@H](c1ccccc1)C(C)(C)C(=O)Nc1ccc(C(F)(F)F)cc1</smiles>

\section{$(R, R)-3$ ha}

Data file E: \DATA WWHYWHM-2-109-124-133-3-13 2019-10-27 21-03-37\WHM-2-109-124-133-3-136.D Sample Name: WHM-3-13-P-CF3-SR-3

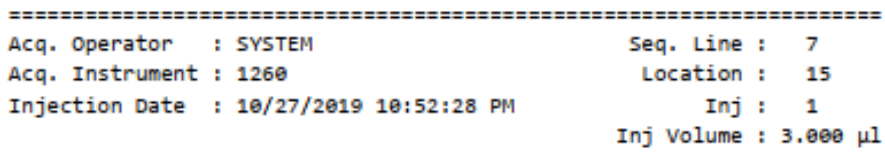

Acq. Method : E: \DATA WWHM\ИHM-2-109-124-133-3-13 2019-10-27 21-03-37\IDH-95-5--3UL-10MIN1 . OML.M

Last changed : 10/27/2019 9:03:37 PM by SYSTEM

Analysis Method: E: \DATA WWHM\ИHM-2-109-124-133-3-13 2019-10-27 21-03-37\IDH-95-5--3UL-10MIN1 . $M L . M$ (Sequence Method)

Last changed : 4/30/2020 4:15:08 PM by SYSTEM (modified after loading)

Additional Info: Peak(s) manually integrated

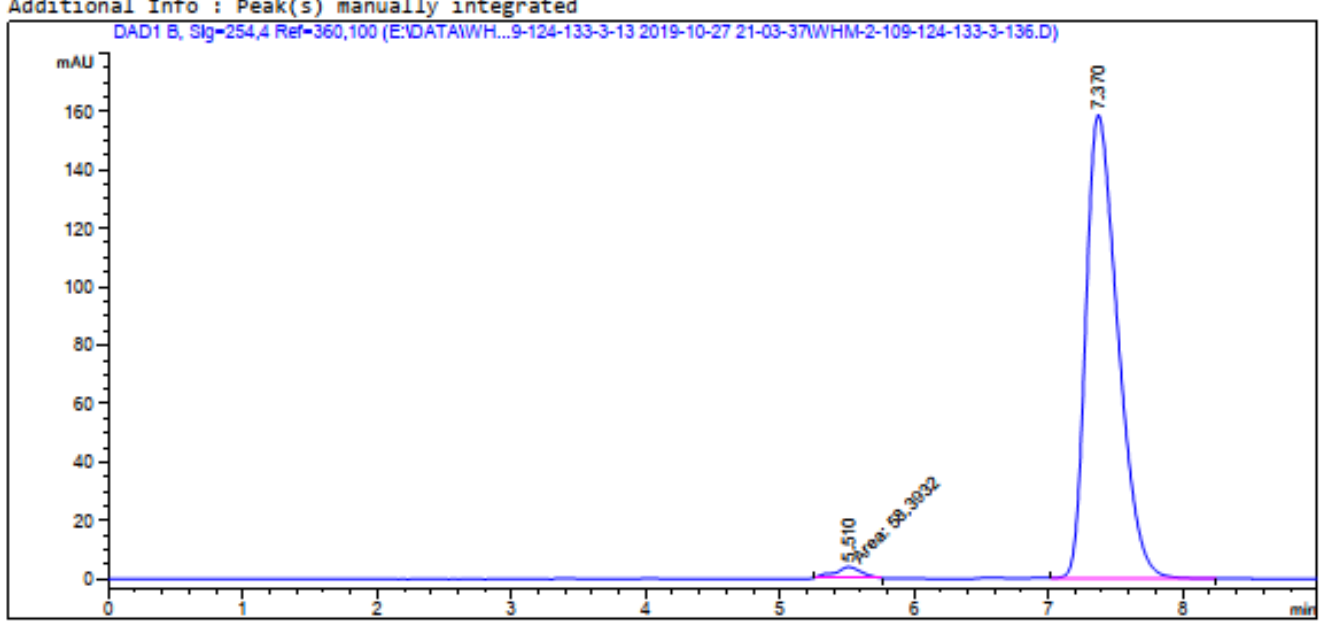

Area Percent Report

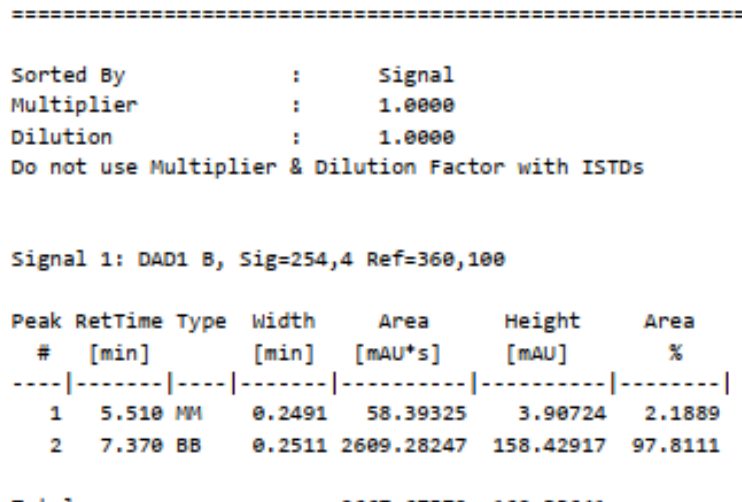

Totals :

$2667.67572 \quad 162.33641$

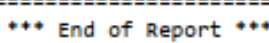


<smiles>C=C[C@H](c1ccccc1)[C@]1(C)COC(c2ccc(C(F)(F)F)cc2)=N1</smiles>

racemic-3ha

Data File E: \DATA WHM-3-28-15 2019-09-e9 21-44-16\WHM-3-15-281.D Sample Name: WHM-3-15-P-CF3-RAC-2

$\begin{array}{lrl}\text { Acq. Operator : SYSTEM } & \text { Seq. Line : } \\ \text { Acq. Instrument : } 1260 & \text { Location : } & 31 \\ \text { Injection Date : } 9 / 9 / 20199: 49: 43 \mathrm{PM} & \text { Inj : } 1\end{array}$

Analysis Method : E: \DATA\WHM-3-28-15 2019-09-09 21-44-16\AD-95-5-28min-5UL-1ML.M (Sequence Method)

Last changed : 10/23/2019 10:22:33 AM by SYSTEM (modified after loading)

Additional Info : Peak(s) manually integrated

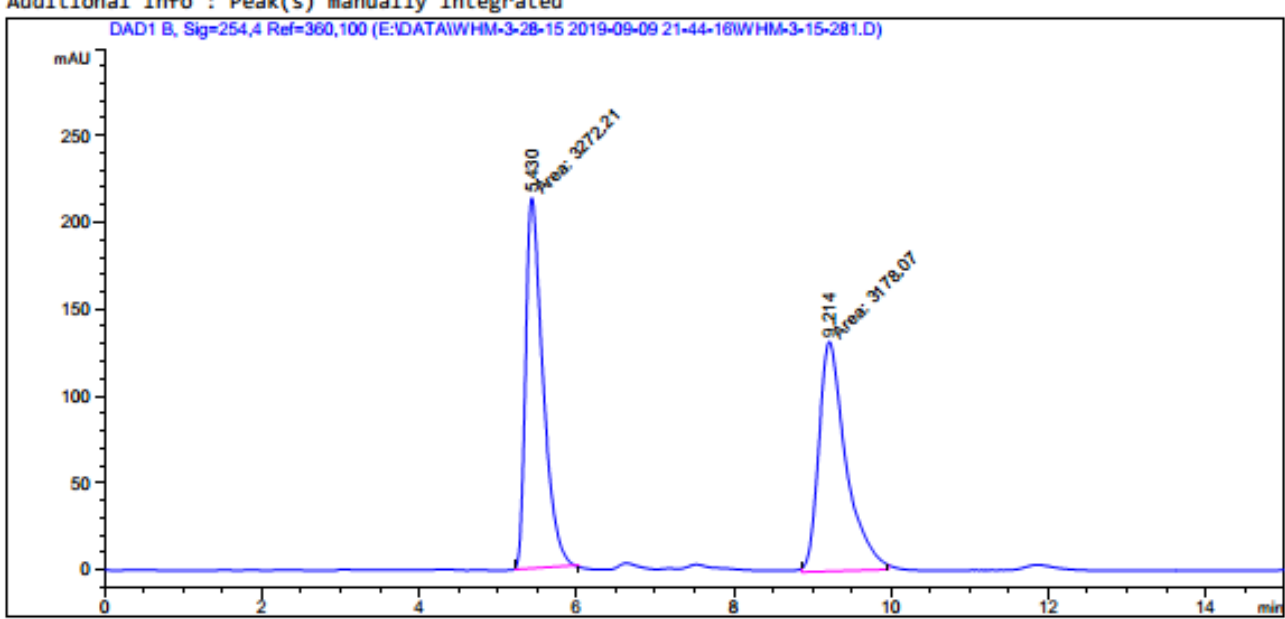

Area Percent Report

$\begin{array}{lll}\text { Sorted By } & : & \text { Signal } \\ \text { Multiplier } & : & 1.0000 \\ \text { Dilution } & : & 1.0000\end{array}$

Do not use Multiplier \& Dilution Factor with ISTDs

Signal 1: DAD1 B, Sig $=254,4$ Ref $=360,100$

\begin{tabular}{|c|c|c|c|c|c|}
\hline $\begin{array}{c}\text { Peak } \\
\#\end{array}$ & $\begin{array}{l}\text { RetTime Type } \\
\text { [min] }\end{array}$ & $\begin{array}{l}\text { Width } \\
\text { [min] }\end{array}$ & $\begin{array}{c}\text { Area } \\
{[\mathrm{mAU} * \mathrm{~s}]}\end{array}$ & $\begin{array}{l}\text { Height } \\
\text { [mAU] }\end{array}$ & $\begin{array}{c}\text { Area } \\
\%\end{array}$ \\
\hline$=1$ & 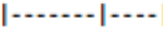 & & 年 & 年 & 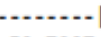 \\
\hline 1 & $5.430 \mathrm{MM}$ & 0.2556 & 3272.20825 & 213.38206 & 50.7297 \\
\hline 2 & $9.214 \mathrm{MM}$ & 0.4013 & 3178.07056 & 131.97913 & 49.2703 \\
\hline$t=2$ & & & 150.2788 & 345.36119 & \\
\hline
\end{tabular}

*** End of Report *** 
<smiles>C=C[C@H](c1ccccc1)[C@]1(C(C)=O)COC(c2ccc(C(F)(F)F)cc2)=N1</smiles>

$(S, R)-3$ ha

Data File E: \DATA WWHMWHM-3-13-15-P-CF3-2 2019-09-08 99-15-57\WHM-3-13-15-23.D Sample Name: WHM-3-15-P-CF3-RR-2

\begin{tabular}{|c|c|c|c|}
\hline Acq. Operator & : SYSTEM & Seq. Line : & 4 \\
\hline Acq. Instrument & : 1260 & Location : & 42 \\
\hline Injection Date & : 9/8/2019 9:55:45 AM & $\begin{array}{r}\text { Inj : } \\
\text { Inj Volume : }\end{array}$ & $\begin{array}{l}: \quad 1 \\
: 5.000 \mu 1\end{array}$ \\
\hline
\end{tabular}

Acq. Method : E: \DATA WHMYWHM-3-13-15-P-CF3-2 2019-09-08 99-15-57\AD-95-5-20min-5UL-1ML.M Last changed : 9/8/2019 9:15:58 AM by SYSTEM

Analysis Method: E: \DATA WWHM WHM-3-13-15-P-CF3-2 2019-09-08 99-15-57\AD-95-5-20min-5UL-1ML.M (Sequence Method)

Last changed : 10/26/2019 8:30:14 PM by SYSTEM

(modified after loading)

Additional Info: Peak(s) manually integrated

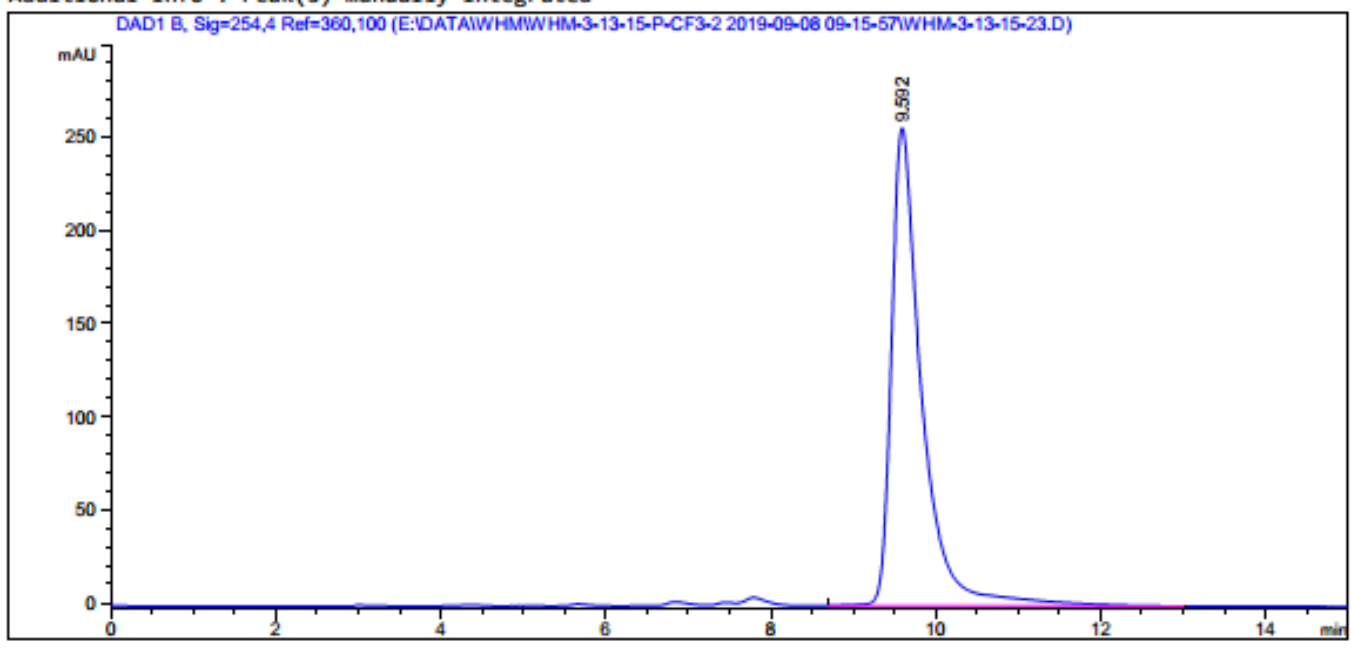

Area Percent Report

Sorted By : : Signal

Multiplier : : 1.0000

Dilution : 1.0000

Do not use Multiplier \& Dilution Factor with ISTDs

Signal 1: DAD1 B, Sig $=254,4$ Ref $=360,108$

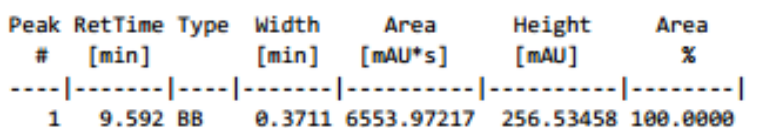

Totals :

$6553.97217 \quad 256.53458$

*** End of Report *** 
<smiles>C=C[C@H](c1ccccc1)C(C)(C)C(=O)N=C(O)c1ccc(C(F)(F)F)cc1</smiles>

\section{$(R, S)-3$ ha}

Data File E: \DATA WHM WHM-3-13-15-P-CF3-2 2019-09-08 99-15-57\WHM-3-13-15-24.D Sample Name: WHM-3-15-P-CF3-SS-2
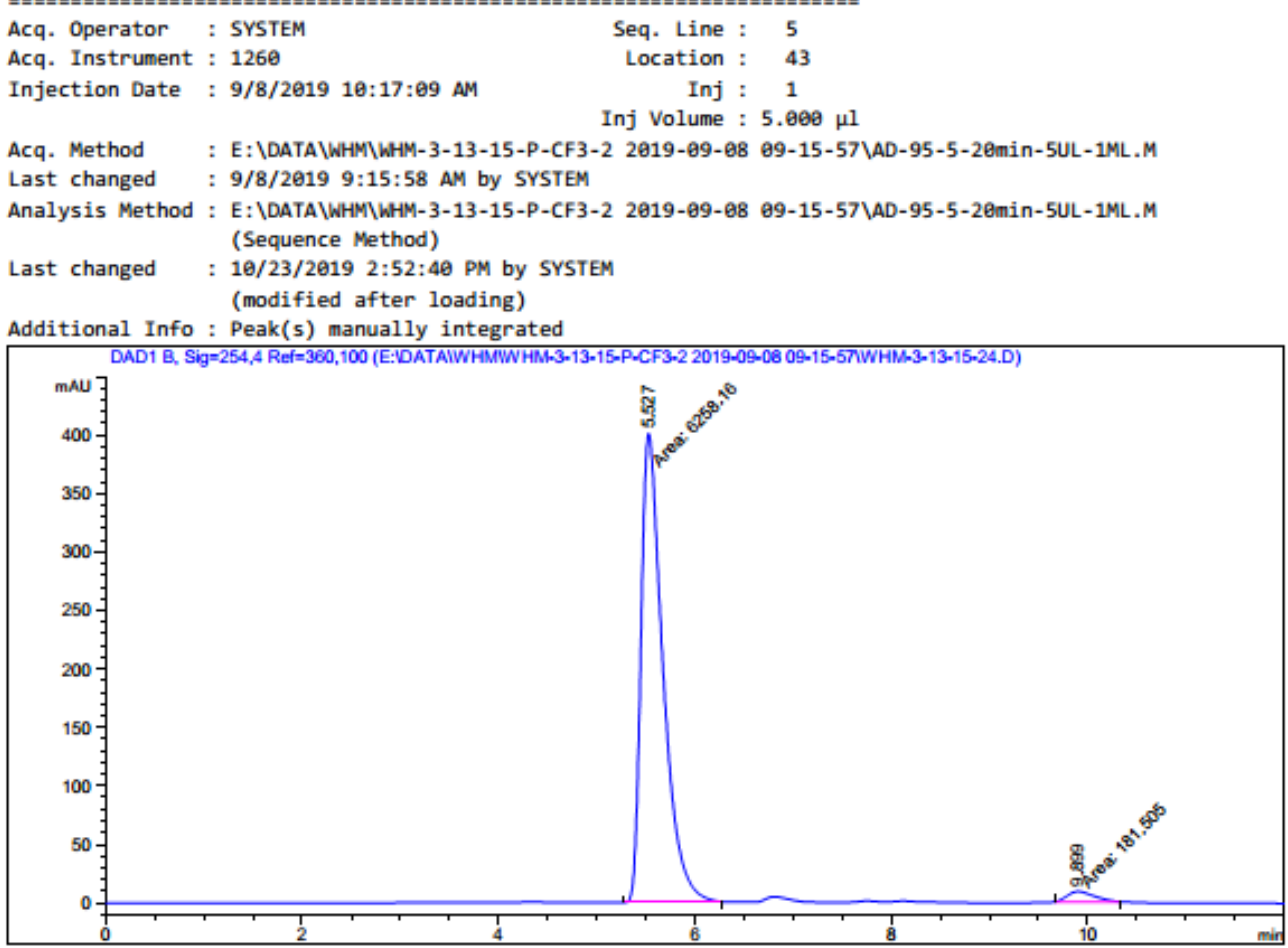

Area Percent Report

$\begin{array}{lll} & & \\ \text { Sorted By } & : & \text { Signal } \\ \text { Multiplier } & : & 1.0000 \\ \text { Dilution } & : & 1.0000\end{array}$

Dilution

Do not use Multiplier \& Dilution Factor with ISTDs

Signal 1: DAD1 B, Sig $=254,4$ Ref $=360,100$

\begin{tabular}{llllll}
$\begin{array}{c}\text { Peak RetTime Type } \\
\text { [min] }\end{array}$ & $\begin{array}{c}\text { Width } \\
\text { [min] }\end{array}$ & $\begin{array}{c}\text { Area } \\
\text { [mAU*s] }\end{array}$ & $\begin{array}{l}\text { Height } \\
\text { [mAU] }\end{array}$ & \multicolumn{1}{c}{$\begin{array}{c}\text { Area } \\
\%\end{array}$} \\
\hline 1 & $5.527 \mathrm{MM}$ & 0.2600 & 6258.16406 & 401.19919 & 97.1815 \\
2 & $9.899 \mathrm{MM}$ & 0.3318 & 181.50507 & 9.11695 & 2.8185 \\
& & & & & \\
Totals : & & 6439.66913 & 410.31614 &
\end{tabular}

*** End of Report *** 


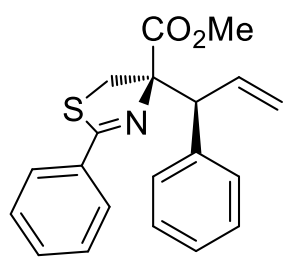

\section{racemic-3ia}

Data file E: \DATA WWM YWHM-3-75-78RAC 2019-10-15 88-40-11 \WHM-3-75-78RAC2.D Sample Name: WHM-3-78-SZ-RAC

\begin{tabular}{|c|c|c|c|}
\hline Acq. Operator & : SYSTEM & Seq. Line : & 3 \\
\hline Acq. Instrument & $: 1260$ & Location : & 52 \\
\hline Injection Date & : 10/15/2019 9:14:06 AM & $\begin{array}{r}\text { Inj : } \\
\text { Inj Volume : }\end{array}$ & $\begin{array}{c}1 \\
5.000\end{array}$ \\
\hline
\end{tabular}

Acq. Method : E: \DATAWWHMYWHM-3-75-78RAC 2019-10-15 98-40-11 \IDH-98-2--1ML-20MIN-5UL .M Last changed : 10/15/2019 8:40:11 AM by SYSTEM

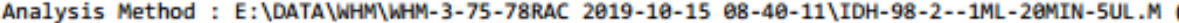
Sequence Method)

Last changed : 10/23/2019 8:47:41 PM by SYSTEM (modified after loading)

Additional Info : Peak(s) manually integrated

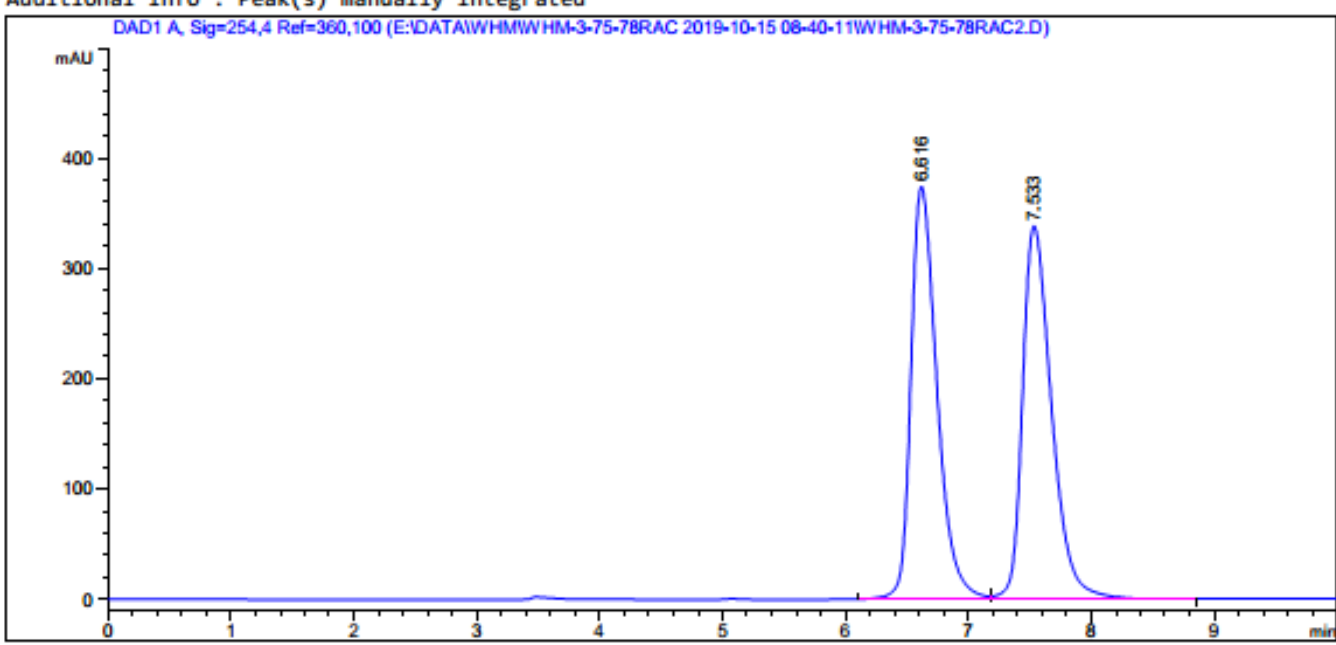

\begin{tabular}{|c|c|c|}
\hline Sorted By & : & Signal \\
\hline Multiplier & : & 1.0000 \\
\hline Dilution & : & 1.0000 \\
\hline
\end{tabular}

Do not use Multiplier \& Dilution Factor with ISTDs

Signal 1: DAD1 A, Sig $=254,4$ Ref $=360,100$

\begin{tabular}{cccccc}
$\begin{array}{c}\text { Peak RetTime Type } \\
\text { \# } \\
\text { [min] }\end{array}$ & $\begin{array}{c}\text { Width } \\
\text { [min] }\end{array}$ & $\begin{array}{c}\text { Area } \\
\text { [mAU*s] }\end{array}$ & $\begin{array}{c}\text { Height } \\
\text { [mAU] }\end{array}$ & $\begin{array}{c}\text { Area } \\
\%\end{array}$ \\
\hline 1 & 6.616 BV & 0.2268 & 5508.80957 & 374.19598 & 49.7160 \\
2 & 7.533 VB & 0.2521 & 5571.74609 & 338.37683 & 50.2840 \\
& & & & & \\
Totals : & & $1.10806 e 4$ & 712.57281 &
\end{tabular}

*** End of Report *** 


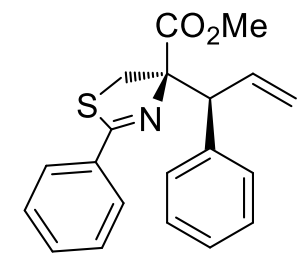

\section{$(R, S)-3$ ia}

Data File E: \DATA WHM WHM-3-75-78RSSR 2019-10-14 20-13-32\WHM-3-75-783.D Sample Name: WHM-3-78-SZ-RS

\begin{tabular}{|c|c|c|c|}
\hline Acq. Operator & : SYSTEM & Seq. Line & : \\
\hline Acq. Instrument & : 1260 & Location & 53 \\
\hline Injection Date & : 10/14/2019 9:09:02 PM & $\begin{array}{r}\text { Inj } \\
\text { Inj Volume }\end{array}$ & $\begin{array}{lc}: & 1 \\
: & 5.000\end{array}$ \\
\hline
\end{tabular}

Acq. Method : E: \DATA WHM WHM-3-75-78RSSR 2019-10-14 20-13-32\IDH-98-2--1ML-20MIN-5UL.M Last changed : 10/14/2019 8:13:32 PM by SYSTEM

Analysis Method : E: \DATA WHM\WHM-3-75-78RSSR 2019-10-14 20-13-32\IDH-98-2--1ML-20MIN-5UL.M ( Sequence Method)

Last changed : 10/23/2019 8:44:37 PM by SYSTEM (modified after loading)

Additional Info: Peak(s) manually integrated

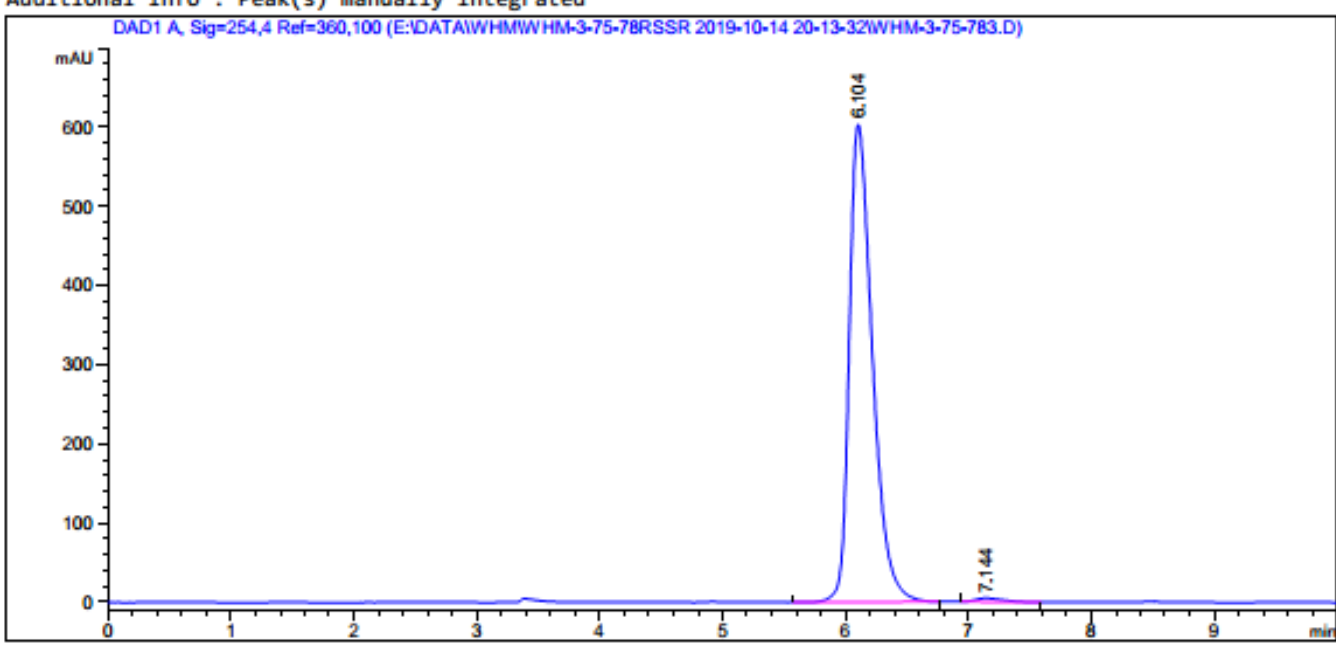

\begin{tabular}{|c|c|c|}
\hline Sorted By & : & Signa \\
\hline Multiplier & : & 1.000 \\
\hline Dilution & : & 1.0000 \\
\hline
\end{tabular}

Do not use Multiplier \& Dilution Factor with ISTDs

Signal 1: DAD1 A, $\operatorname{Sig}=254,4$ Ref $=360,100$

\begin{tabular}{|c|c|c|c|c|c|}
\hline $\begin{array}{c}\text { Peak } \\
\#\end{array}$ & $\begin{array}{l}\text { RetTime Type } \\
\text { [min] }\end{array}$ & $\begin{array}{l}\text { Width } \\
\text { [min] }\end{array}$ & $\begin{array}{c}\text { Area } \\
{\left[\mathrm{mAU}^{*} \mathrm{~s}\right]}\end{array}$ & $\begin{array}{l}\text { Height } \\
\text { [mAU] }\end{array}$ & $\begin{array}{c}\text { Area } \\
\text { \% }\end{array}$ \\
\hline$\ldots-1$ & $|\cdots+\ldots| \cdots$ & ........ & | & - & - \\
\hline 1 & $6.104 \mathrm{BB}$ & 0.2009 & 7903.39551 & 603.16797 & 99.1682 \\
\hline 2 & $7.144 \mathrm{BB}$ & 0.2038 & 66.29429 & 4.42589 & 0.8318 \\
\hline & & & 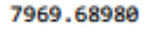 & 386 & \\
\hline
\end{tabular}

*** End of Report *** 


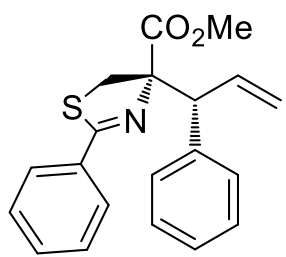

$(S, R)-3$ ia

Data File E: \DATA WHM WHM-3-75-78RSSR 2019-10-14 20-13-32\WHM-3-75-784.D Sample Name: WHM-3-78-SZ-SR

\begin{tabular}{|c|c|c|c|}
\hline Acq. Operator & : SYSTEM & Seq. Line : & 5 \\
\hline Acq. Instrument & : 1260 & Location : & 54 \\
\hline Injection Date & : 10/14/2019 9:30:32 PM & $\begin{array}{r}\text { Inj : } \\
\text { Inj Volume : }\end{array}$ & $\begin{array}{c}1 \\
5.00\end{array}$ \\
\hline
\end{tabular}

Acq. Method : E: DDATA WHMYWHM-3-75-78RSSR 2019-10-14 20-13-32\IDH-98-2--1ML-20MIN-5UL.M Last changed : 10/14/2019 8:13:32 PM by SYSTEM

Analysis Method: E: \DATA WWMM WHM-3-75-78RSSR 2019-10-14 20-13-32\IDH-98-2--1ML-20MIN-5UL.M Sequence Method)

Last changed : 10/23/2019 8:46:13 PM by SYSTEM

(modified after loading)

Additional Info: Peak(s) manually integrated

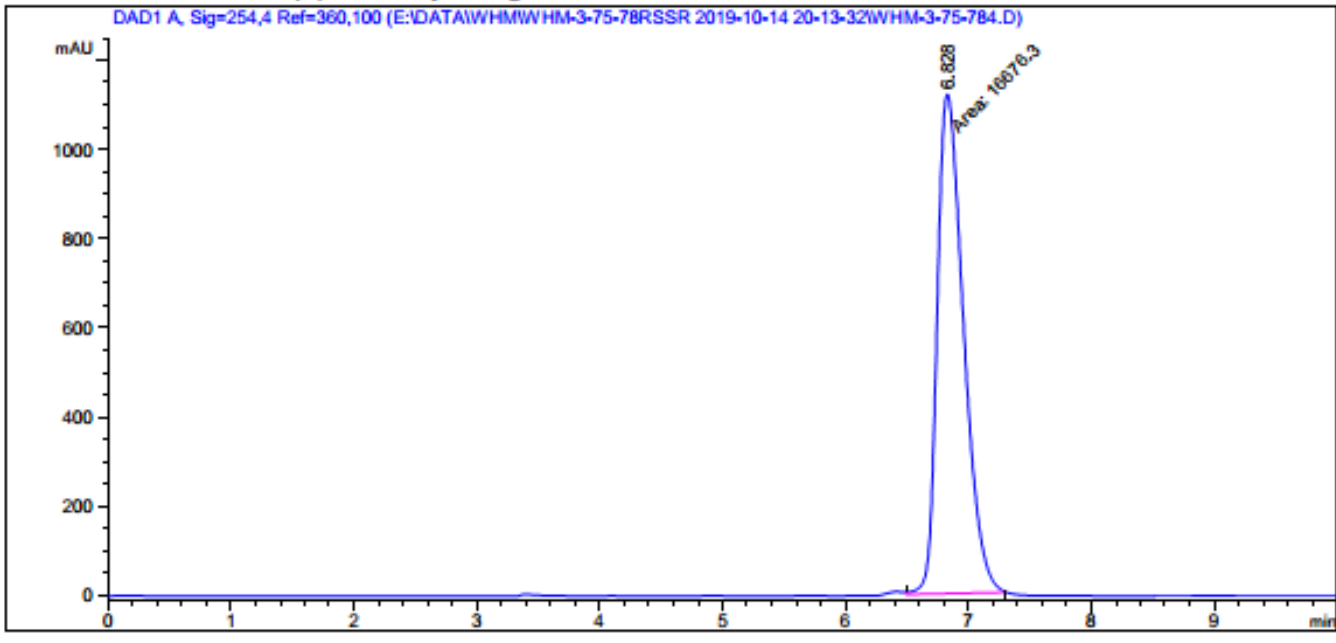

Area Percent Report

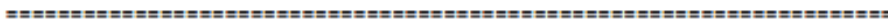

Sorted By : Signal

Multiplier : : 1.0000

Dilution : 1.0000

Do not use Multiplier \& Dilution Factor with ISTDs

Signal 1: DAD1 A, Sig $=254,4$ Ref $=360,100$

\begin{tabular}{|c|c|c|c|c|c|}
\hline $\begin{array}{c}\text { Peak } \\
\#\end{array}$ & $\begin{array}{l}\text { RetTime Type } \\
\text { [min] }\end{array}$ & $\begin{array}{l}\text { Width } \\
\text { [min] }\end{array}$ & $\begin{array}{c}\text { Area } \\
{\left[\mathrm{mAU}^{*} \mathrm{~s}\right]}\end{array}$ & $\begin{array}{l}\text { Height } \\
\text { [mAU] }\end{array}$ & $\begin{array}{c}\text { Area } \\
\text { \% }\end{array}$ \\
\hline 1 & $6.828 \mathrm{MM}$ & .2485 & $1.66763 \mathrm{e} 4$ & 118.40259 & 308 \\
\hline
\end{tabular}

Totals : $\quad 1.66763 \mathrm{e} 4 \quad 1118.49259$

*** End of Report *** 


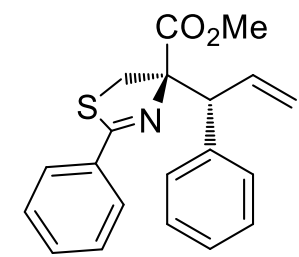

\section{racemic-3ia}

Data File E: \DATA WHM\WHM-3-83RAC-84RSSR 2019-10-19 19-43-67\WHM-3-83RAC-84RSSR1.D Sample Name: WHM-3-83-SZ-RAC
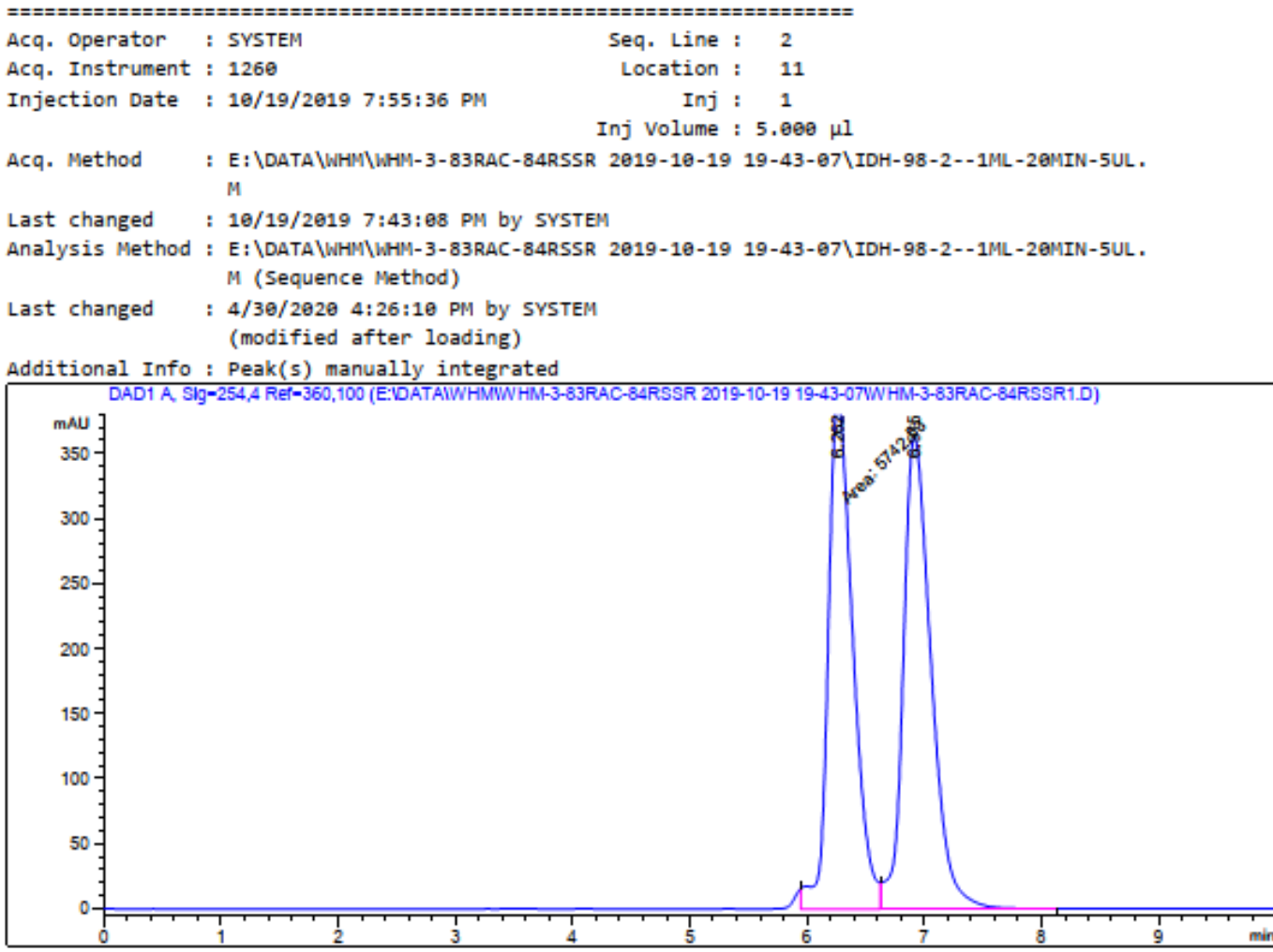

Area Percent Report

\begin{tabular}{|c|c|c|c|c|}
\hline Sorted By & $:$ & \multicolumn{3}{|l|}{ signal } \\
\hline \multirow{2}{*}{$\begin{array}{l}\text { Multiplier } \\
\text { Dilution }\end{array}$} & $:$ & \multicolumn{3}{|l|}{1.0000} \\
\hline & : & \multicolumn{3}{|l|}{$\begin{array}{l}1.0000 \\
1.0000\end{array}$} \\
\hline \multicolumn{5}{|c|}{ Do not use Multiplier \& Dilution Factor with ISTDs } \\
\hline \multicolumn{5}{|c|}{ Signal 1: DAD1 A, $\operatorname{Sig}=254,4$ Ref $=360,100$} \\
\hline Peak RetTime Type & Width & Area & Height & Area \\
\hline | & $|-\ldots|$ & $|-2 .-2 .-1|$ & {$[\mathrm{mAU}]$} & $x^{x}$ \\
\hline $16.262 \mathrm{FM}$ & 0.2458 & 5742.53076 & 389.40225 & 48.8686 \\
\hline $6.915 \mathrm{VB}$ & 0.2520 & 6008.43066 & 363.13577 & 51.1314 \\
\hline
\end{tabular}

*** End of Report *** 


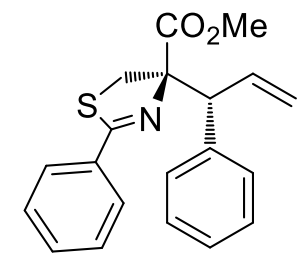

\section{$(R, R)-3$ ia}

Data File E: \DATA WWMYWHM-3-83-SZ-RRSS 2019-10-19 08-54-54 \whm-3-83-RRSS1.D Sample Name: WHM-3-83-SZ-RR

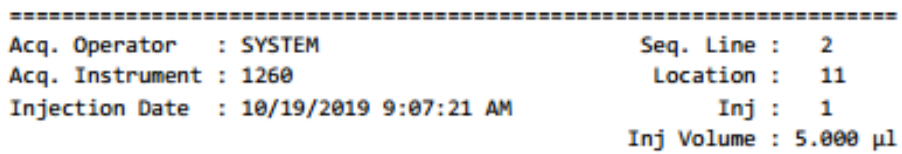

Acq. Method : E: \DATA WHMIWHM-3-83-SZ-RRSS 2019-10-19 08-54-54 IDH-98-2--1ML-20MIN-5UL.M Last changed : 10/19/2019 $8: 54: 54$ AM by SYSTEM

Analysis Method : E: \DATA WHM\WHM-3-83-SZ-RRSS 2019-10-19 68-54-54\IDH-98-2--1ML-20MIN-5UL.M (Sequence Method)

Last changed : 10/23/2019 8:49:26 PM by SYSTEM (modified after loading)

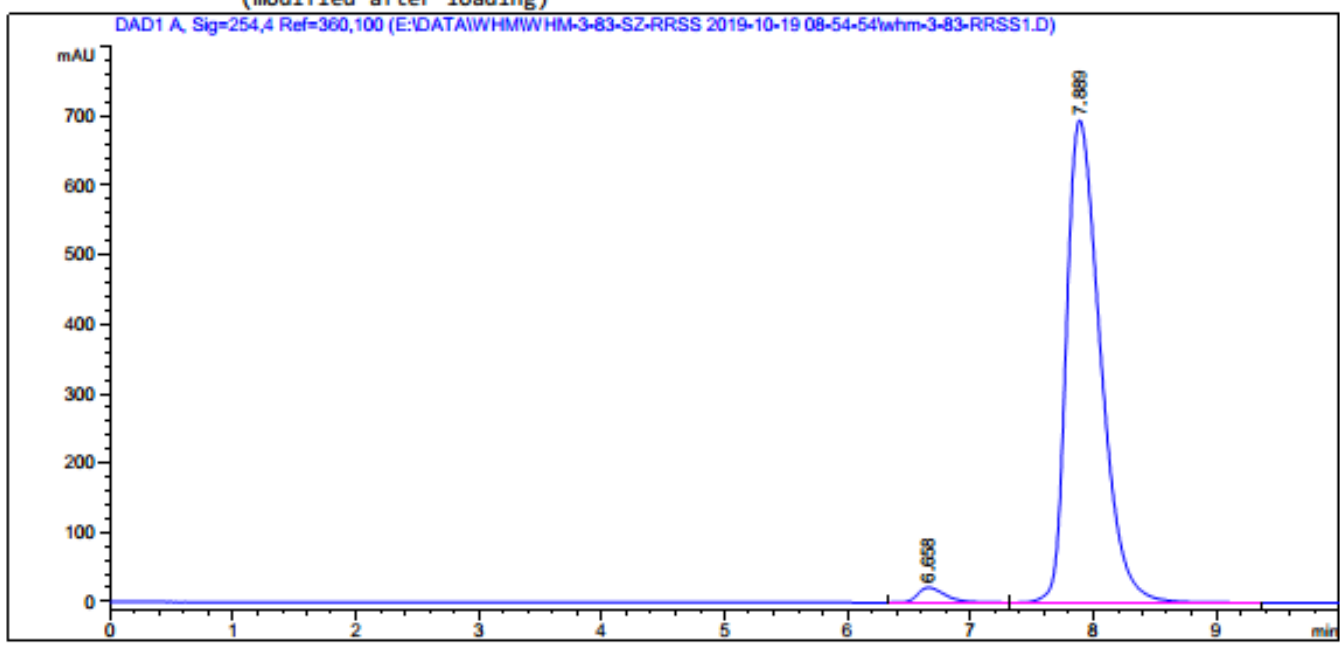

Area Percent Report

$\begin{array}{lll} & & \\ \text { Sorted By } & : & \text { Signal } \\ \text { Multiplier } & : & 1.0000 \\ \text { Dilution } & : & 1.0000\end{array}$

Do not use Multiplier \& Dilution Factor with ISTDs

Signal 1: DAD1 A, Sig $=254,4$ Ref $=360,100$

\begin{tabular}{|c|c|c|c|c|c|}
\hline $\begin{array}{c}\text { Peak } \\
\#\end{array}$ & $\begin{array}{l}\text { RetTime Type } \\
\text { [min] }\end{array}$ & $\begin{array}{l}\text { Width } \\
\text { [min] }\end{array}$ & $\begin{array}{c}\text { Area } \\
{\left[\mathrm{mAU}^{*} \mathrm{~s}\right]}\end{array}$ & $\begin{array}{l}\text { Height } \\
\text { [mAU] }\end{array}$ & $\begin{array}{c}\text { Area } \\
\text { \% }\end{array}$ \\
\hline t & i & 1 & | & | & 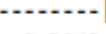 \\
\hline 1 & $6.658 \mathrm{BB}$ & 0.2441 & 345.18863 & 21.64238 & 2.5263 \\
\hline 2 & $7.889 \mathrm{BB}$ & 0.2985 & $1.33187 \mathrm{e} 4$ & 695.14417 & 97.4737 \\
\hline Tot & : & & $1.36639 \mathrm{e} 4$ & 716.78654 & \\
\hline
\end{tabular}

*** End of Report *** 


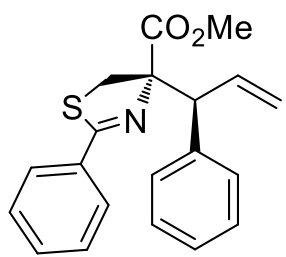

$(S, S)-3 i a$

Data file E: \DATA WHM WWH-3-83-SZ-RRSS 2019-10-19 08-54-54 \whm-3-83-RRSS2.D Sample Name: WHM-3-83-SZ-SS

\begin{tabular}{|c|c|c|c|}
\hline Acq. Operator & : SYSTEM & Seq. Line : & 3 \\
\hline Acq. Instrument & : 1260 & Location : & 12 \\
\hline Injection Date & : 10/19/2019 9:28:47 AM & $\begin{array}{r}\text { Inj : } \\
\text { ij Volume : }\end{array}$ & $\begin{array}{l}1 \\
.00 \ell\end{array}$ \\
\hline
\end{tabular}

Acq. Method : E: \DATAWWHMWHM-3-83-SZ-RRSS 2019-10-19 98-54-54\IDH-98-2--1ML-20MIN-5UL.M Last changed : 10/19/2019 8:54:54 AM by SYSTEM

Analysis Method : E: \DATA WHMIWHM-3-83-SZ-RRSS 2019-10-19 08-54-54\IDH-98-2--1ML-20MIN-5UL.M (Sequence Method)

Last changed : 10/23/2019 8:49:26 PM by SYSTEM

(modified after loading)

Additional Info : Peak(s) manually integrated

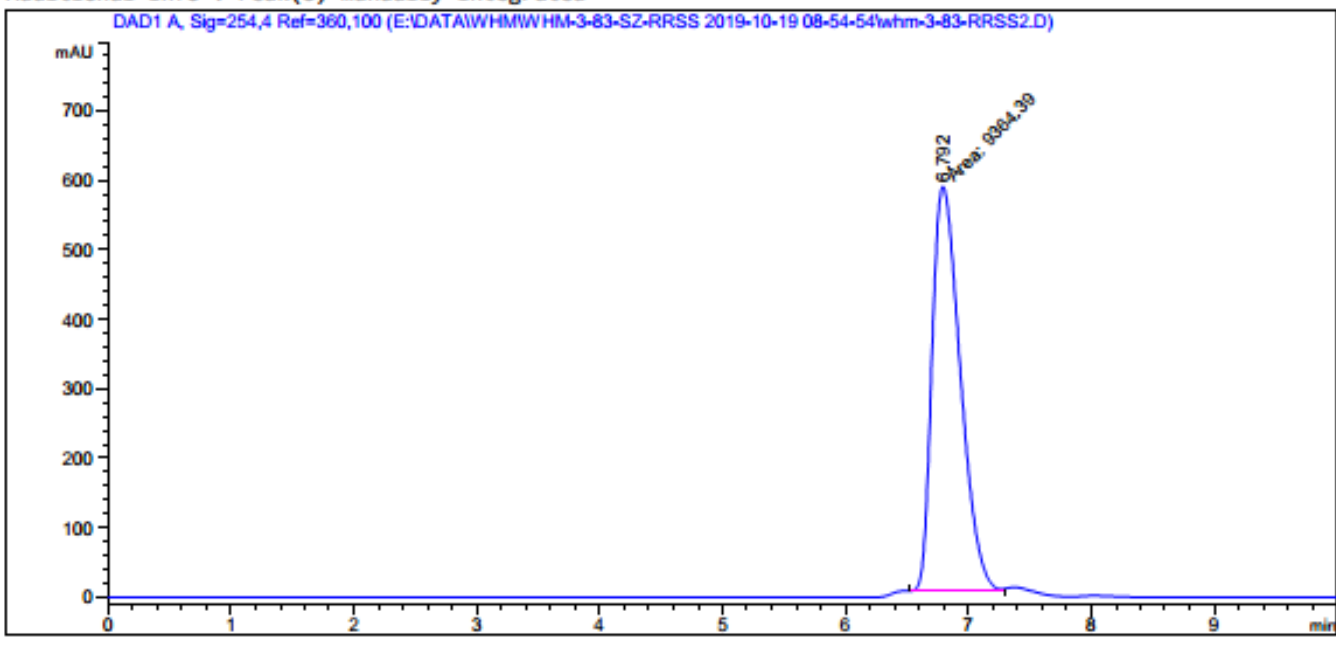

Area Percent Report

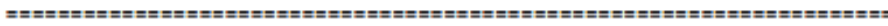

Sorted By : Signal

Multiplier $\quad: \quad 1.0000$

Dilution : 1.0000

Do not use Multiplier \& Dilution Factor with ISTDs

Signal 1: DAD1 A, Sig $=254,4$ Ref $=360,100$

\begin{tabular}{|c|c|c|c|c|c|}
\hline $\begin{array}{c}\text { Peak } \\
\#\end{array}$ & $\begin{array}{l}\text { RetTime Type } \\
\text { [min] }\end{array}$ & $\begin{array}{l}\text { Width } \\
\text { [min] }\end{array}$ & $\begin{array}{c}\text { Area } \\
{\left[\mathrm{mAU}^{*} \mathrm{~s}\right]}\end{array}$ & $\begin{array}{l}\text { Height } \\
\text { [mAU] }\end{array}$ & $\begin{array}{c}\text { Area } \\
\text { \% }\end{array}$ \\
\hline
\end{tabular}

Totals :

$9364.39063 \quad 583.96912$

*** End of Report *** 
<smiles>C=CC(c1ccc(C)cc1)C(C)(COC(C)=O)NC(=O)c1ccc(C(F)(F)F)cc1</smiles>

racemic-3hb

Data file E: \DATA WHM WWH-3-113-BU-PH-BN-SR 2019-11-13 16-48-22\WHM-3-113-BU-PH-BN-SR5.D Sample Name: WHM-3-111-P-ME-RAC

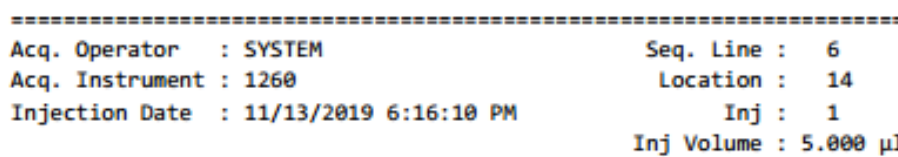

Acq. Method : E: \DATAWWHMWHM-3-113-BU-PH-BN-SR 2019-11-13 16-48-22\IDH-98-2--1ML-20MINSUL.M

Last changed : 11/13/2019 5:37:16 PM by SYSTEM

Analysis Method : E: (DATA WWHMTWHM-3-113-BU-PH-BN-SR 2019-11-13 16-48-22\IDH-98-2--1ML-20MINSUL.M (Sequence Method)

Last changed : 11/13/2019 9:43:59 PM by SYSTEM (modified after loading)

Additional Info : Peak(s) manually integrated

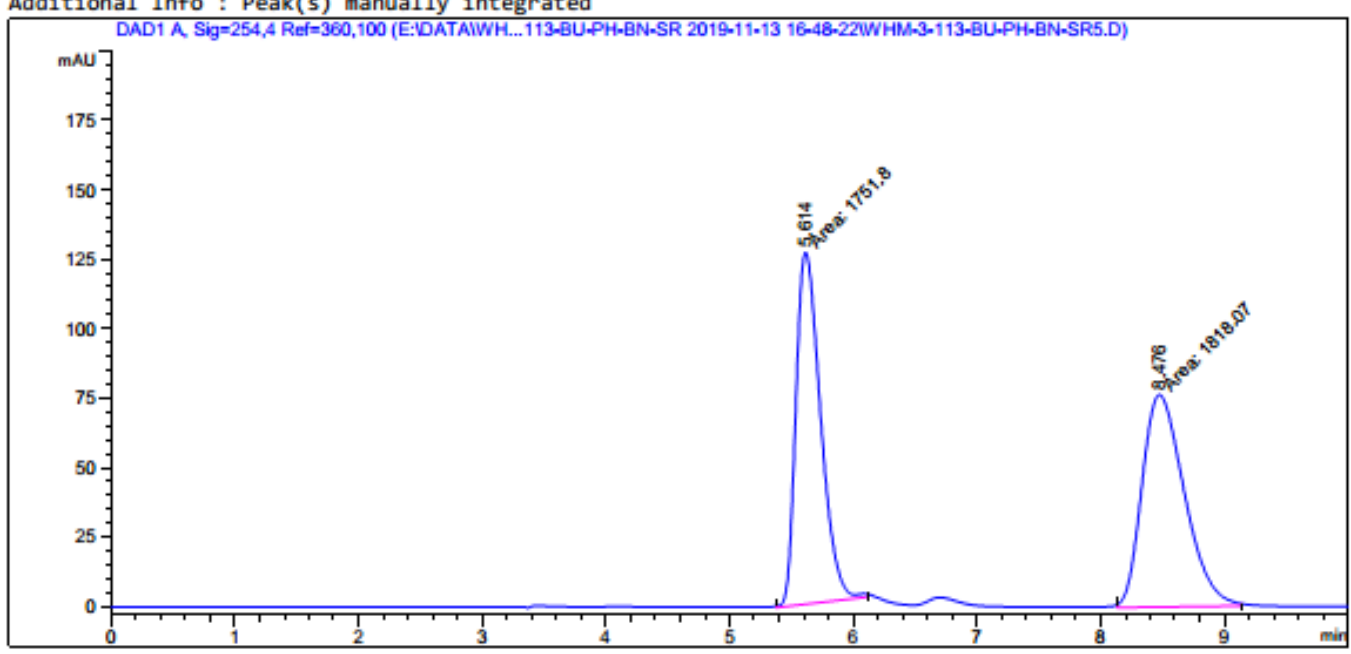

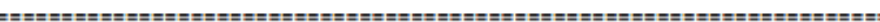

Area Percent Report

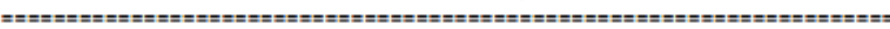

$\begin{array}{lll}\text { Sorted By } & : & \text { Signal } \\ \text { Multiplier } & : & 1.0000 \\ \text { Dilution } & : & 1.0000\end{array}$

Do not use Multiplier \& Dilution Factor with ISTDs

Signal 1: DAD1 A, Sig $=254,4$ Ref $=360,100$

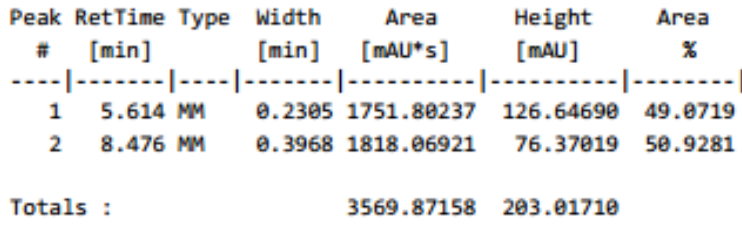

*** End of Report *** 
<smiles>C=CC(c1ccc(C)cc1)C(C)(COC(C)=O)NC(=O)c1ccc(C(F)(F)F)cc1</smiles>

$(S, S)-3 h b$

Data file E: \DATA WHM \WH-3-103-111-112 2019-11-13 09-11-00\WHM-3-103-HBW-RAC-111-1123.D Sample Name: WHM-3-111-P-ME-RS

\begin{tabular}{|c|c|c|c|}
\hline Acq. Operator & : SYSTEM & Seq. Line : & 4 \\
\hline Acq. Instrument & : 1260 & Location : & 12 \\
\hline Injection Date & : 11/13/2019 9:50:50 AM & $\begin{array}{r}\text { Inj : } \\
\text { Inj Volume : }\end{array}$ & $\begin{array}{lc}: & 1 \\
: & 5.000 \mu \mathrm{l}\end{array}$ \\
\hline
\end{tabular}

Acq. Method : E: \DATA WWHMYHM-3-103-111-112 2019-11-13 09-11-00\IDH-98-2--1ML-20MIN-5UL.M Last changed : 11/13/2019 9:11:00 AM by SYSTEM

Analysis Method: E: \DATA WHM WHM-3-103-111-112 2019-11-13 09-11-00\IDH-98-2--1ML-20MIN-5UL.M (Sequence Method)

Last changed : 11/13/2019 9:47:33 PM by SYSTEM

(modified after loading)

Additional Info: Peak(s) manually integrated

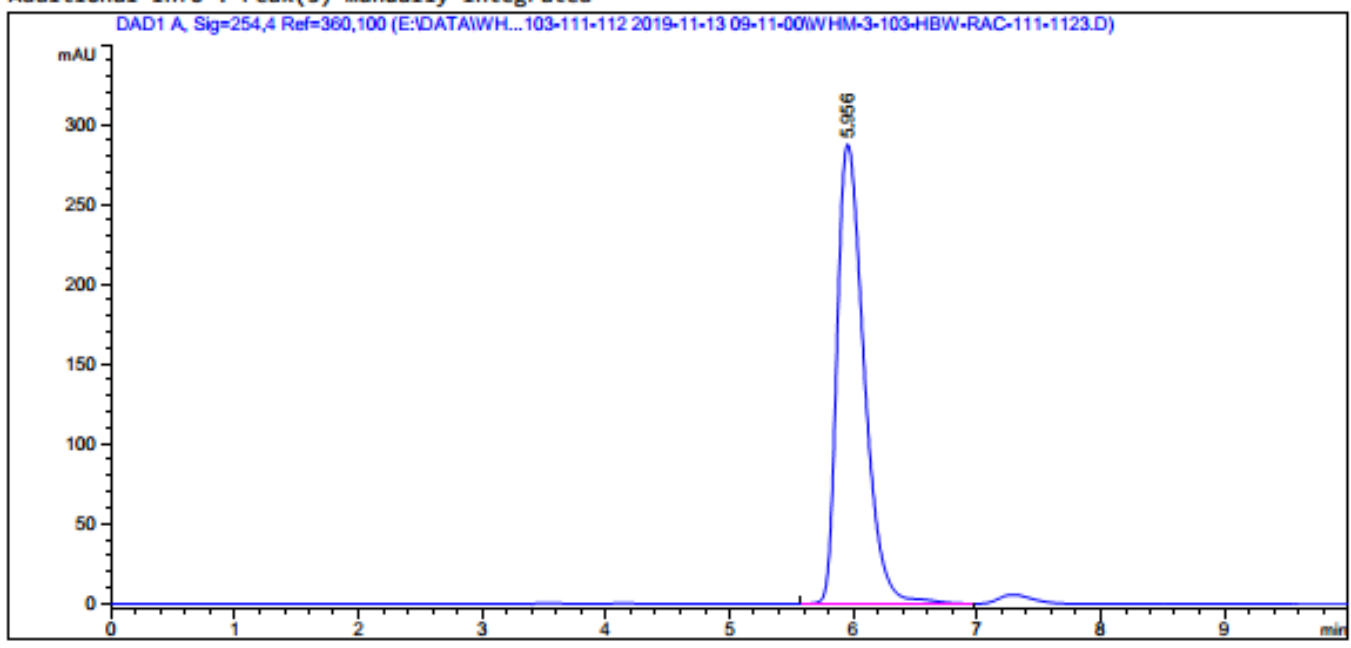

Area Percent Report

Sorted By : Signal

Multiplier : $\quad 1.0000$

Dilution 1.0000

Do not use Multiplier \& Dilution Factor with ISTDs

Signal 1: DAD1 A, Sig $=254,4$ Ref $=360,100$

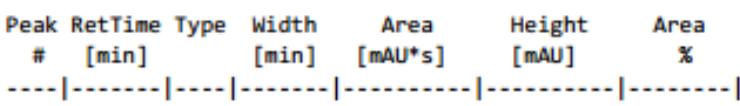

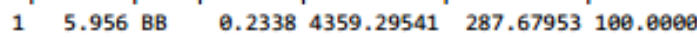

Totals :

$4359.29541 \quad 287.67953$

*** End of Report *** 
<smiles>C=CC(c1ccc(Cl)cc1)C(C)(COC)N1CCOC1c1ccc(C(F)(F)F)cc1</smiles>

racemic-3hc

Data File E: \DATA WHMWWHM-3-42-43-RAC 2019-09-21 99-19-22\WHM-3-42-43-RAC1.D Sample Name: WHM-3-42-P-CL-RAC

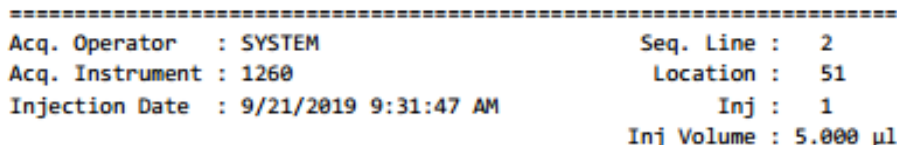

Acq. Method : E: \DATA WHM WHM-3-42-43-RAC 2019-69-21 99-19-22\AD-95-5-20min-5UL-1ML.M Last changed : 9/21/2019 9:19:22 AM by SYSTEM

Analysis Method : E: \DATA WHM WHM-3-42-43-RAC 2019-09-21 99-19-22\AD-95-5-20min-5UL-1ML.M ( Sequence Method)

Last changed : 10/23/2019 7:34:43 PM by SYSTEM

(modified after loading)

Additional Info : Peak(s) manually integrated

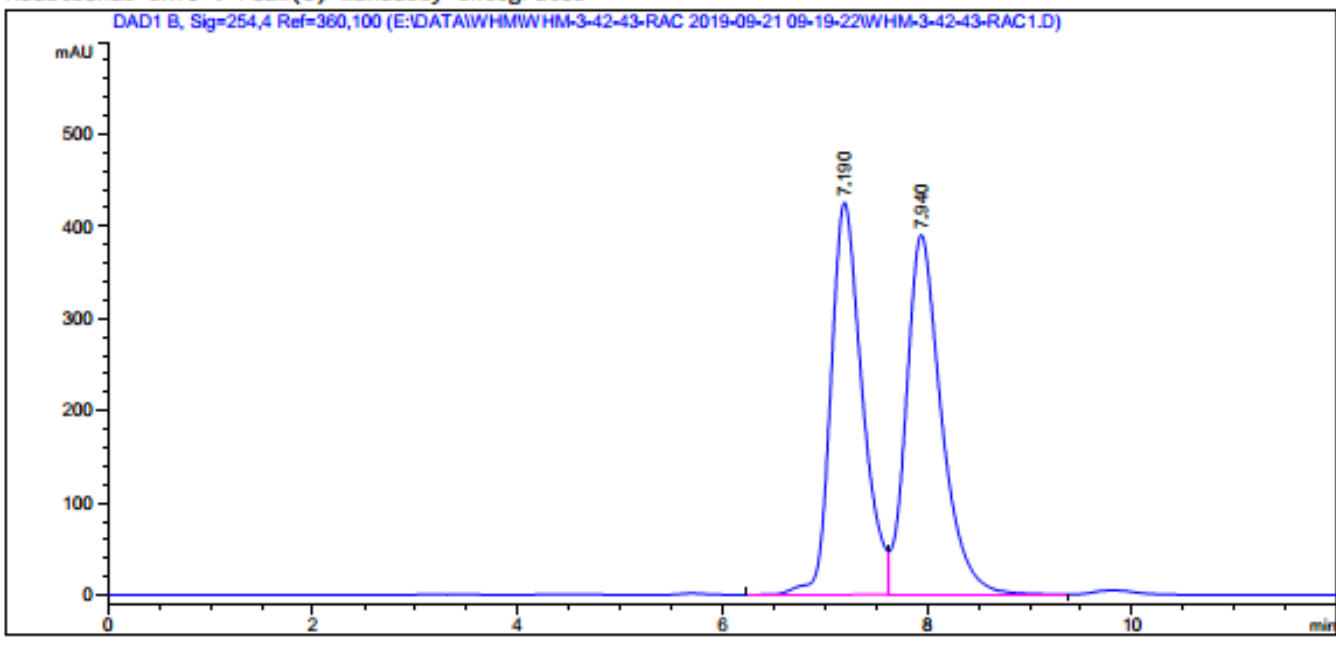

Area Percent Report

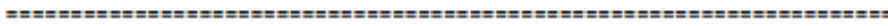

Sorted By : Signal

Multiplier : : 1.0000

Dilution : 1.0000

Do not use Multiplier \& Dilution Factor with ISTDs

Signal 1: DAD1 B, Sig $=254,4$ Ref $=360,100$

\begin{tabular}{|c|c|c|c|c|c|}
\hline $\begin{array}{c}\text { Peak } \\
\#\end{array}$ & $\begin{array}{l}\text { RetTime Type } \\
\text { [min] }\end{array}$ & $\begin{array}{l}\text { Width } \\
\text { [min] }\end{array}$ & $\begin{array}{c}\text { Area } \\
{\left[\mathrm{mAU}^{*} \mathrm{~s}\right]}\end{array}$ & $\begin{array}{l}\text { Height } \\
\text { [mAU] }\end{array}$ & $\begin{array}{c}\text { Area } \\
\text { \% }\end{array}$ \\
\hline-1 & $\cdots$ & & 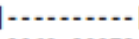 & - & . \\
\hline 1 & $7.190 \mathrm{BV}$ & 0.3244 & 9062.80078 & 425.69269 & 49.3207 \\
\hline 2 & $7.940 \mathrm{VB}$ & 0.3533 & 9312.45020 & 390.43909 & 50.6793 \\
\hline Tota] & & & $1.83753 \mathrm{e} 4$ & 816.13177 & \\
\hline
\end{tabular}

*** End of Report *** 
<smiles>C=CC(c1ccc(Cl)cc1)[C@]1(C)COC(c2ccc(C(F)(F)F)cc2)=N[C@@H]1C(C)=O</smiles>

(S, S)-3hc

Data file E: DDATA WHM \WHM-3-42-43 2019-09-20 98-13-40\WHM-3-42-431.D Sample Name: WHM-3-42-P-CL-RS
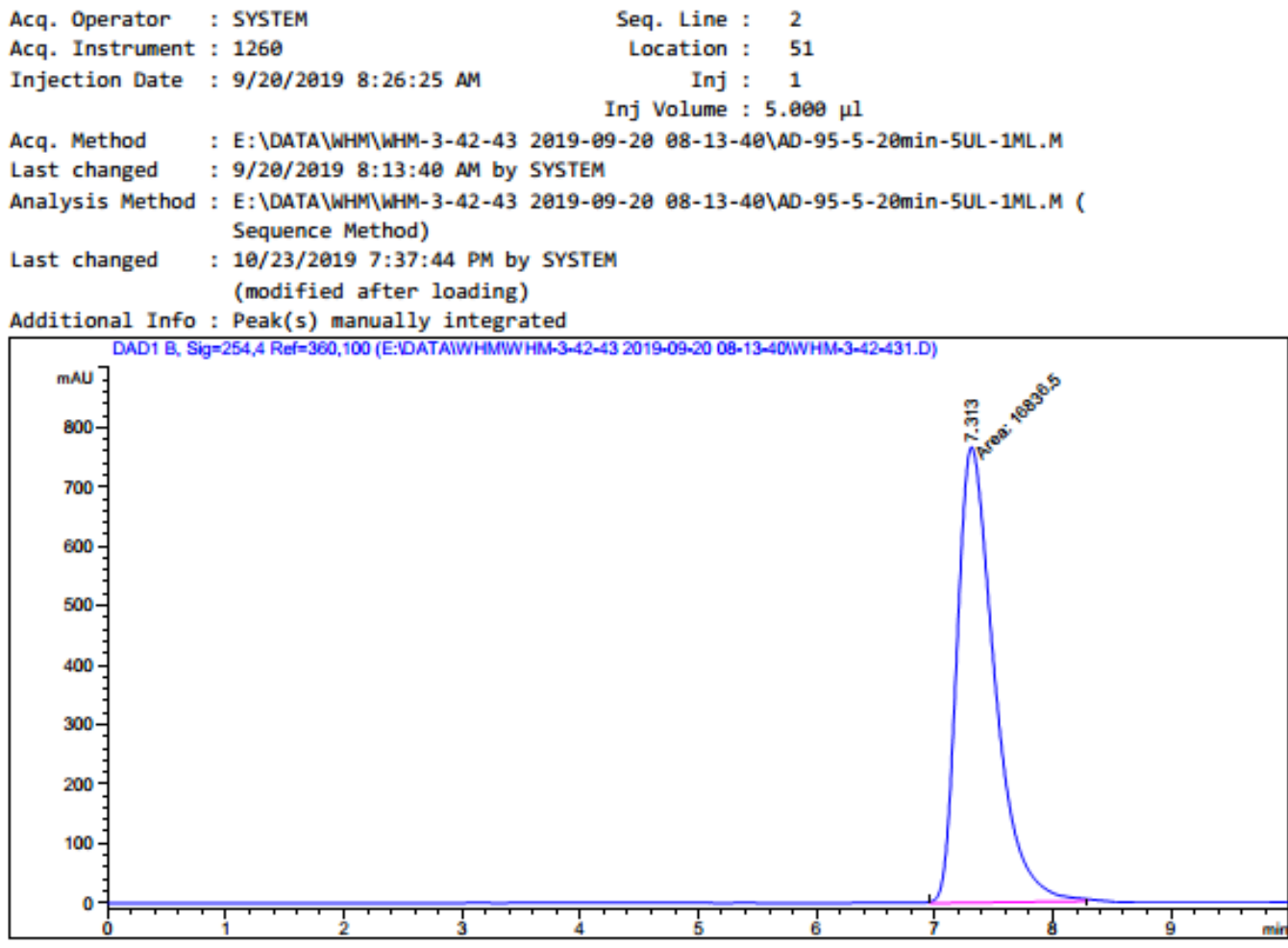

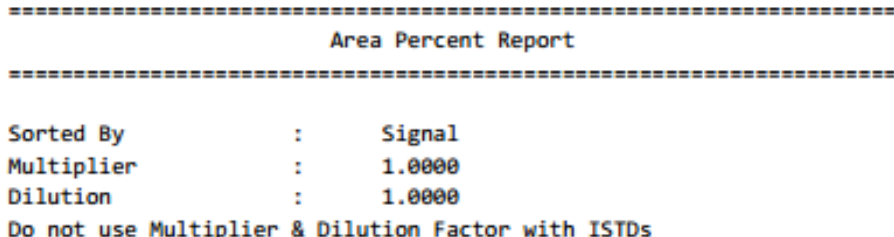

Signal 1: DAD1 B, Sig $=254,4$ Ref $=360,100$

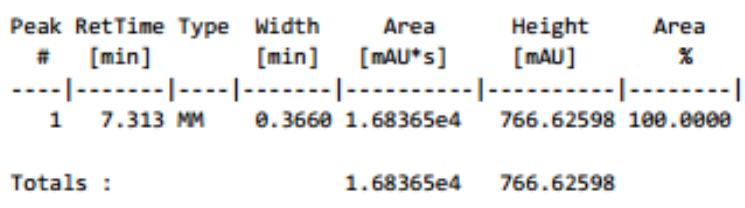

*** End of Report *** 
<smiles>C=CC(c1ccc(OC)cc1)C(C)(C)C1=NC(c2ccc(C(F)(F)F)cc2)=CC1</smiles>

racemic-3hd

Data file E: \DATA WHM WHM-3-42-43-RAC 2019-09-21 99-19-22\WHM-3-42-43-RAC2.D Sample Name: WHM-3-43-P-OME-RAC
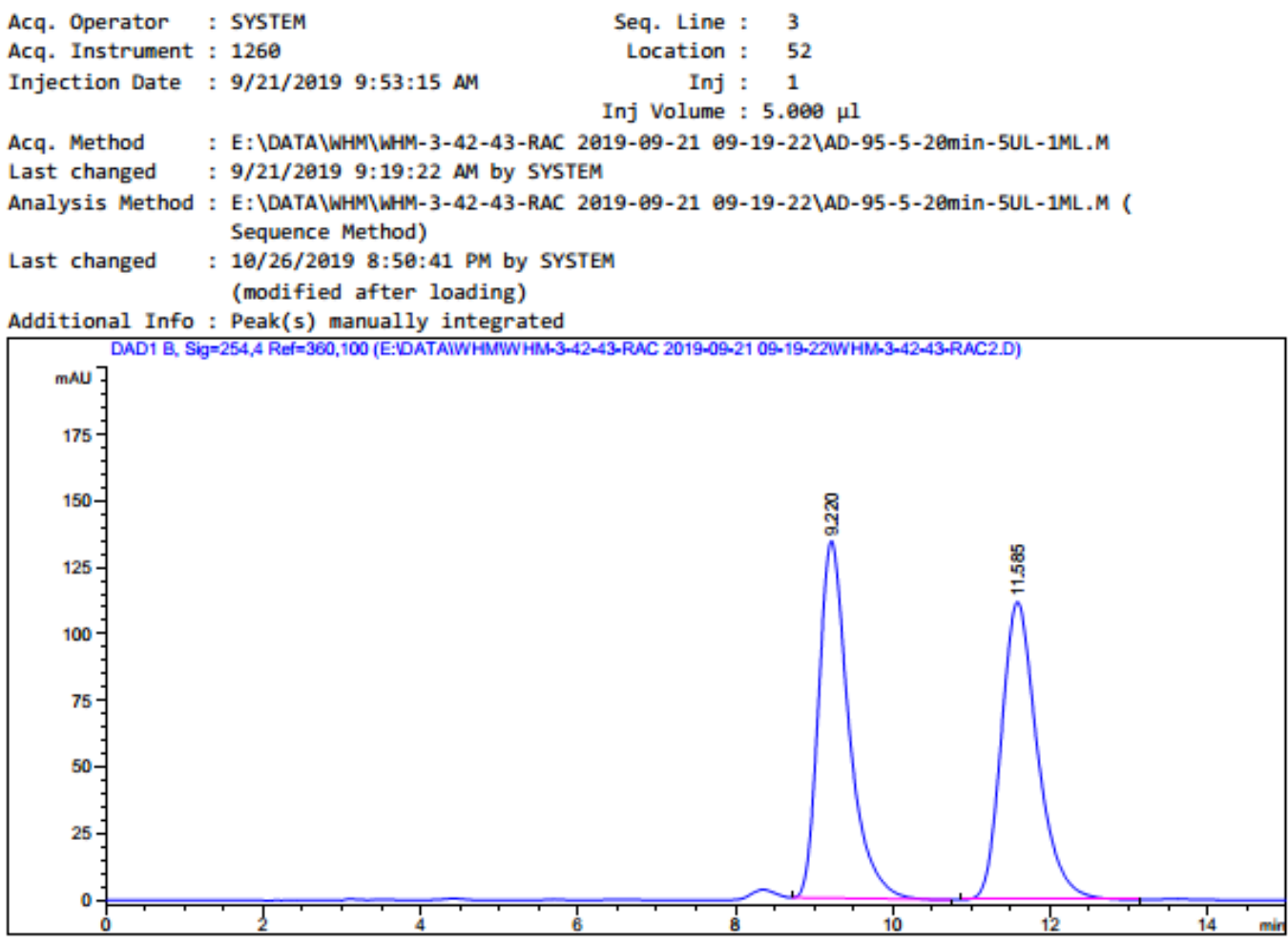

Area Percent Report

\begin{tabular}{|c|c|c|}
\hline Sorted By & : & Signal \\
\hline Multiplier & : & 1.0800 \\
\hline Dilution & : & 1.0000 \\
\hline
\end{tabular}

Dilution - $1.0 \mathrm{BO}$

Do not use Multiplier \& Dilution Factor with ISTDs

Signal 1: DAD1 B, $\operatorname{Sig}=254,4$ Ref $=360,100$

\begin{tabular}{|c|c|c|c|c|c|}
\hline $\begin{array}{c}\text { Peak } \\
\#\end{array}$ & $\begin{array}{l}\text { RetTime Type } \\
\text { [min] }\end{array}$ & $\begin{array}{l}\text { Width } \\
\text { [min] }\end{array}$ & $\begin{array}{c}\text { Area } \\
{\left[\mathrm{mAU}^{*} \mathrm{~s}\right]}\end{array}$ & $\begin{array}{l}\text { Height } \\
\text { [mAU] }\end{array}$ & $\begin{array}{c}\text { Area } \\
\text { \% }\end{array}$ \\
\hline 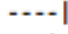 & 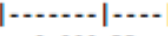 & & 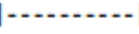 & . & - \\
\hline 1 & $9.220 \mathrm{BB}$ & 0.4038 & 3603.16089 & 134.38486 & 50.2653 \\
\hline 2 & $11.585 \mathrm{BB}$ & 0.4754 & 3565.12573 & 111.99984 & 49.7347 \\
\hline Tota & & & 7168.28662 & 246.38470 & \\
\hline
\end{tabular}

*** End of Report *** 
<smiles>C=C[C@H](c1ccc(OC)cc1)[C@]1(C(C)=O)COC(c2ccc(C(F)(F)F)cc2)=N1</smiles>

$(S, S)-3 h d$

Data file E: \DATA WHM\WHM-3-42-43 2019-09-20 98-13-40\WHM-3-42-433.D Sample Name: WHM-3-43-P-OME-RS

\begin{tabular}{|c|c|c|c|}
\hline $\begin{array}{l}\text { Acq. Operator: } \\
\text { Acq. Instrument : }\end{array}$ & $\begin{array}{l}: \text { SYSTEM } \\
: 1260\end{array}$ & $\begin{array}{l}\text { Seq. Line } \\
\text { Location : }\end{array}$ & $\begin{array}{l}4 \\
53\end{array}$ \\
\hline Injection Date : & : 9/20/2019 9:89:25 AM & Inj & $\begin{array}{c}1 \\
2.96\end{array}$ \\
\hline
\end{tabular}

Acq. Method : E: \DATA

Last changed : 9/20/2019 8:13:40 AM by SYSTEM

Analysis Method: E: \DATA WHM\WHM-3-42-43 2019-09-20 08-13-40\AD,95-5,2UL, 1ML, 30MIN.M ( Sequence Method)

Last changed : 10/23/2019 7:39:45 PM by SYSTEM

(modified after loading)

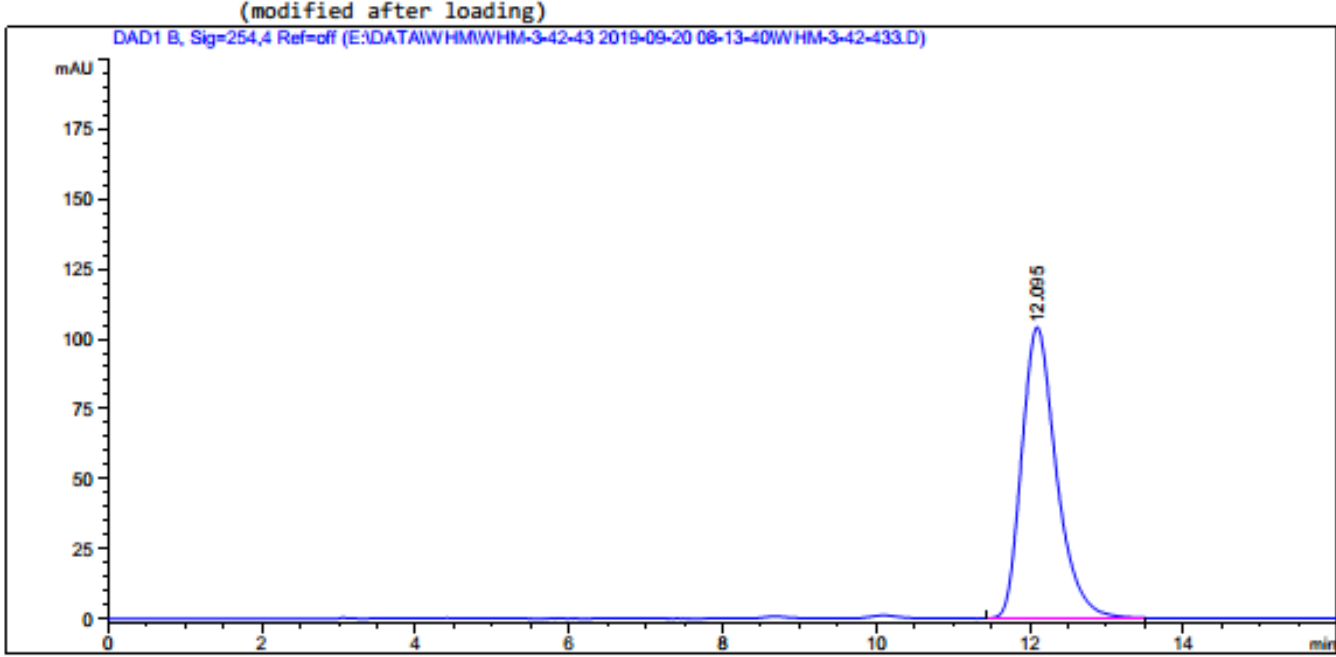

Area Percent Report

\begin{tabular}{|c|c|}
\hline Sorted By & $:$ \\
\hline Multiplier & : \\
\hline Dilution & : \\
\hline
\end{tabular}

Do not use Multiplier \& Dilution Factor with ISTDs

Signal 1: DAD1 B, Sig $=254,4$ Ref $=$ off

\begin{tabular}{l} 
Peak RetTime Type Width $\begin{array}{c}\text { Area } \\
\text { [min] } \\
\text { [mAU*s] }\end{array}$ [min] [mAU] \\
\hdashline
\end{tabular}

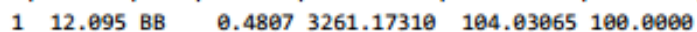

Totals : $\quad 3261.17310 \quad 104.03065$ 
<smiles>C=CC(c1ccc(Br)cc1)C(C)(C)NC(=O)c1ccc(C(F)(F)F)cc1</smiles>

racemic-3he

Data File E: \DATA WHM WHM-3-46-54-RSSRRAC 2019-99-26 14-49-93\WHM-3-47-54-RAC6.D Sample Name: WHM-3-52-P-BR-RAC

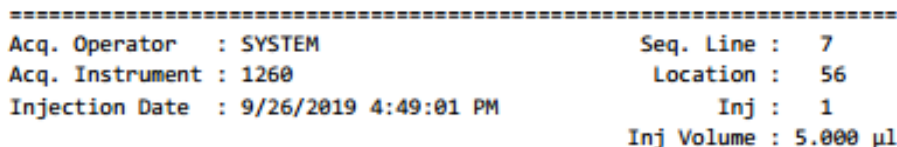

Acq. Method : E: \DATA WHMYWHM-3-46-54-RSSRRAC 2019-09-26 14-49-03\IDH-98-2--1ML-20MIN-5UL

Last changed $\quad$ : 9/26/2019 2:49:03 PM by SYSTEM

Analysis Method : E: \DATA WHMThHM-3-46-54-RSSRRAC 2019-09-26 14-49-03\IDH-98-2--1ML-20MIN-5UL .M (Sequence Method)

Last changed : 10/23/2019 8:04:22 PM by SYSTEM (modified after loading)

Additional Info: Peak(s) manually integrated

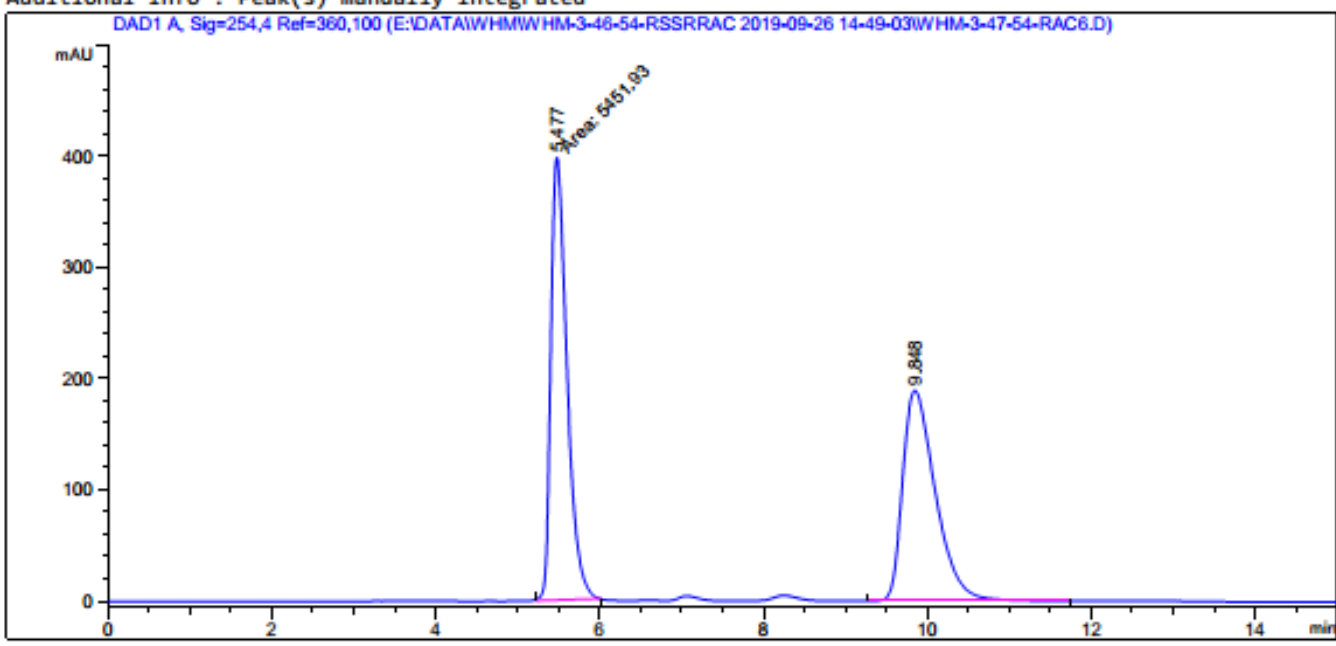

Area Percent Report

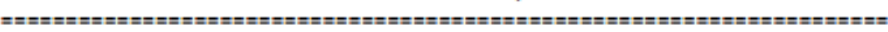

$\begin{array}{llc}\text { Sorted By } & : & \text { Signal } \\ \text { Multiplier } & : & 1.0000 \\ \text { Dilution } & : & 1.0000\end{array}$

Do not use Multiplier \& Dilution Factor with ISTDs

Signal 1: DAD1 A, Sig $=254,4$ Ref $=360,100$

\begin{tabular}{cccccc}
$\begin{array}{c}\text { Peak RetTime Type } \\
\text { [min] }\end{array}$ & $\begin{array}{c}\text { Width } \\
\text { [min] }\end{array}$ & $\begin{array}{c}\text { Area } \\
\text { [mAU*s] }\end{array}$ & $\begin{array}{l}\text { Height } \\
\text { [mAU] }\end{array}$ & $\begin{array}{c}\text { Area } \\
\%\end{array}$ \\
\hline 1 & $5.477 \mathrm{MM}$ & 0.2282 & 5451.92725 & 398.18448 & 50.8947 \\
2 & $9.848 \mathrm{BB}$ & 0.4253 & 5260.23828 & 189.24489 & 49.1053 \\
& & & & & \\
Totals : & & $1.07122 \mathrm{e} 4$ & 587.42937
\end{tabular}

*** End of Report *** 
<smiles>C=CC(c1ccc(Br)cc1)C(C)(C)NC(=O)c1ccc(C(F)(F)F)cc1</smiles>

$(S, S)-3$ he

Data file E: DDATA WHM \WHM-3-51-52 2019-09-24 17-28-59\WHM-3-51-522.D Sample Name: WHM-3-52-P-BR-RS

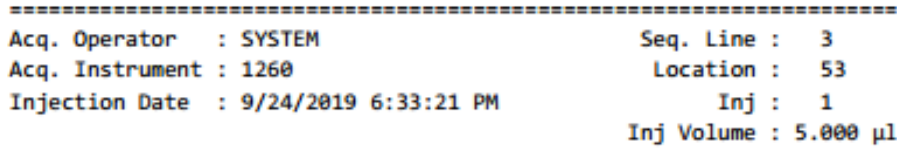

Acq. Method : E: \DATAWWHM\WHM-3-51-52 2019-69-24 17-28-59\ID-98-2-3emin-5UL.M

Last changed : 9/24/2019 5:28:59 PM by SYSTEM

Analysis Method : E: \DATA WHMYWHM-3-51-52 2019-09-24 17-28-59\ID-98-2-38min-5UL.M (Sequence Method)

Last changed : 10/23/2019 4:37:50 PM by SYSTEM

(modified after loading)

Additional Info : Peak(s) manually integrated

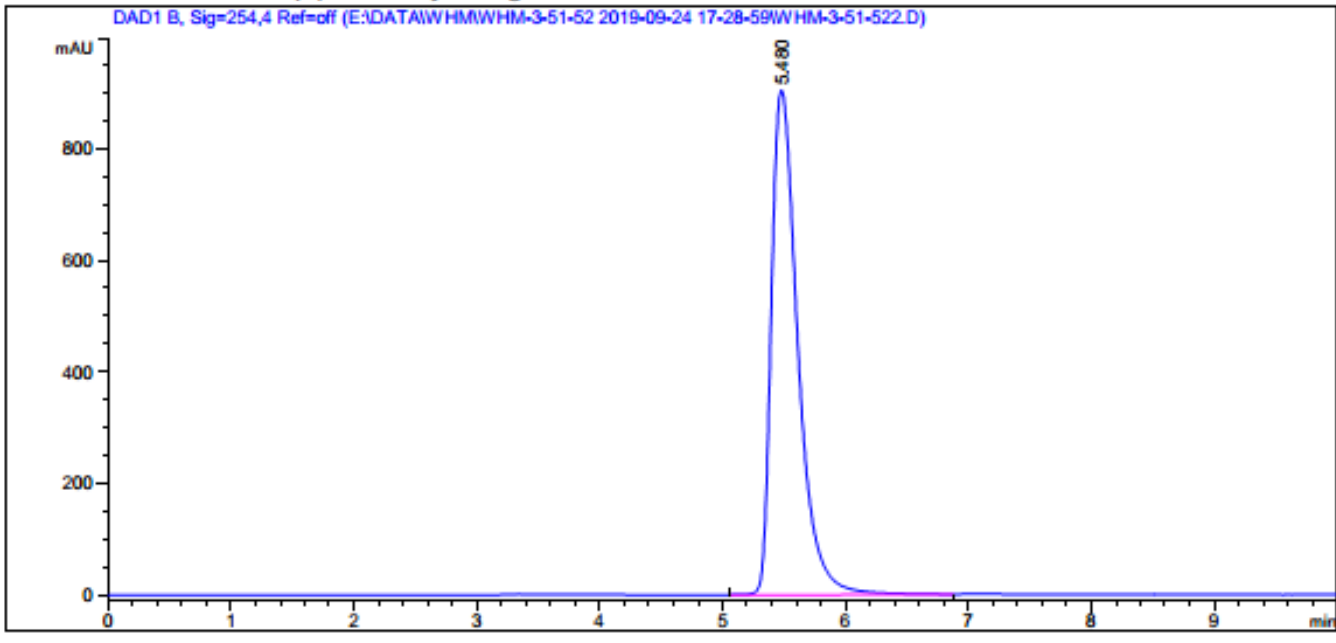

Area Percent Report

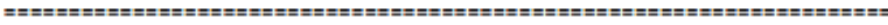

Sorted By : : Signal

Multiplier : : 1.0000

Dilution : 1.0000

Do not use Multiplier \& Dilution Factor with ISTDs

Signal 1: DAD1 B, Sig=254,4 Ref=off

\begin{tabular}{|c|c|c|c|c|c|}
\hline $\begin{array}{c}\text { Peak } \\
\#\end{array}$ & $\begin{array}{l}\text { RetTime Type } \\
\text { [min] }\end{array}$ & $\begin{array}{l}\text { Width } \\
\text { [min] }\end{array}$ & $\begin{array}{c}\text { Area } \\
{\left[\mathrm{mAU}^{*} \mathrm{~s}\right]}\end{array}$ & $\begin{array}{l}\text { Height } \\
\text { [mAU] }\end{array}$ & $\begin{array}{c}\text { Area } \\
\text { \% }\end{array}$ \\
\hline
\end{tabular}

Totals : $\quad 1.35425 \mathrm{e} 4 \quad 965.75879$

*** End of Report *** 
<smiles>C=C[C@H](c1ccc(Cl)c(Cl)c1)[C@]1(C(C)=O)COC1c1ccc(C(F)(F)F)cc1</smiles>

racemic-3hf

Data File E: \DATA WHMWWHM-3-46-54-RSSRRAC 2019-99-26 14-49-93\WHM-3-47-54-RAC7.D Sample Name: WHM-3-53-M,P-CL-RAC

\begin{tabular}{|c|c|c|c|}
\hline Acq. Operator & : SYSTEM & Seq. Line : & 8 \\
\hline Acq. Instrument & : 1260 & Location : & 57 \\
\hline Injection Date & : 9/26/2019 5:10:33 PM & $\begin{array}{r}\text { Inj : } \\
\text { Inj Volume : }\end{array}$ & 1 \\
\hline
\end{tabular}

Inj Volume : $5.000 \mu 1$

Acq. Method : E: \DATA WHMYWHM-3-46-54-RSSRRAC 2019-09-26 14-49-03\IDH-98-2--1ML-20MIN-5UL

Last changed : 9/26/2019 2:49:03 PM by SYSTEM

Analysis Method : E: (DATA WHMTWHM-3-46-54-RSSRRAC 2019-09-26 14-49-03\IDH-98-2--1ML-20MIN-5UL .M (Sequence Method)

Last changed : 10/23/2019 8:06:10 PM by SYSTEM

(modified after loading)

Additional Info: Peak(s) manually integrated

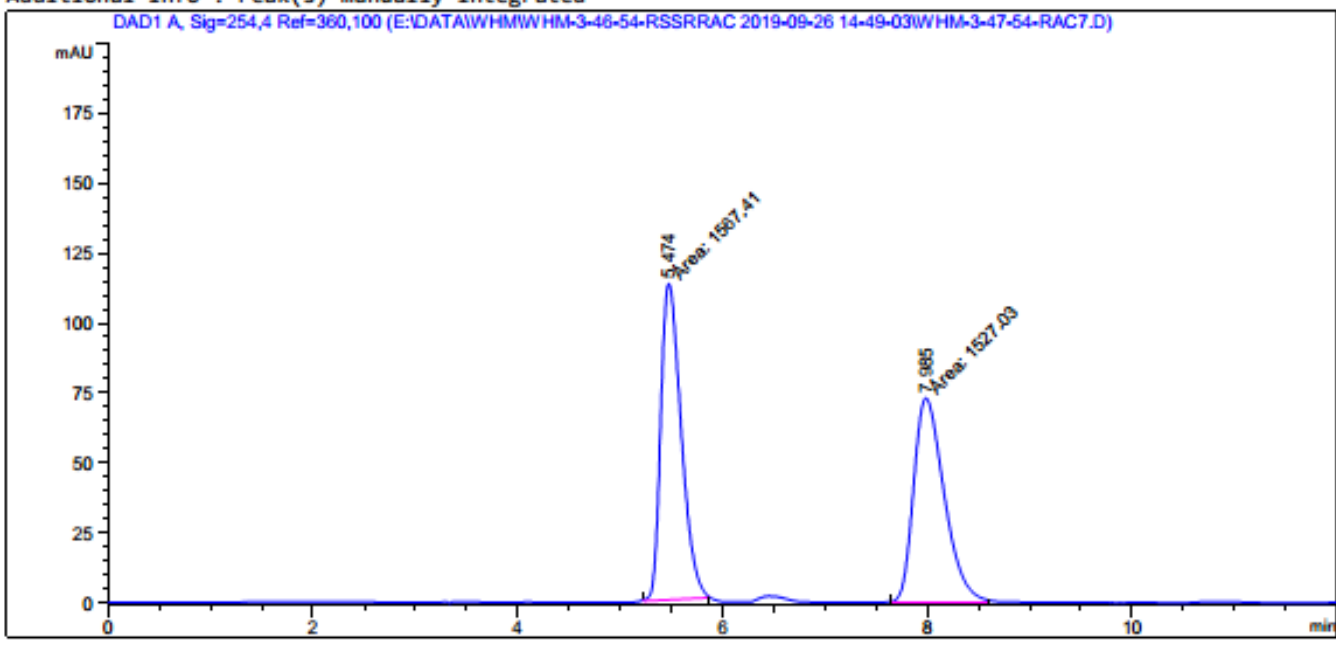

Area Percent Report

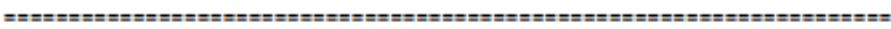

$\begin{array}{llc}\text { Sorted By } & : & \text { Signal } \\ \text { Multiplier } & : & 1.0000 \\ \text { Dilution } & : & 1.0000\end{array}$

Do not use Multiplier \& Dilution Factor with ISTDs

Signal 1: DAD1 A, Sig $=254,4$ Ref $=360,100$

\begin{tabular}{cccccc}
$\begin{array}{l}\text { Peak RetTime Type } \\
\text { [min] }\end{array}$ & $\begin{array}{c}\text { Width } \\
\text { [min] }\end{array}$ & $\begin{array}{c}\text { Area } \\
\text { [mAU*s] }\end{array}$ & $\begin{array}{c}\text { Height } \\
\text { [mAU] }\end{array}$ & $\begin{array}{c}\text { Area } \\
\%\end{array}$ \\
\hline 1 & $5.474 \mathrm{MM}$ & 0.2311 & 1567.41089 & 113.06055 & 50.6525 \\
2 & $7.985 \mathrm{MM}$ & 0.3479 & 1527.02551 & 73.16455 & 49.3475 \\
& & & & & \\
Totals : & & 3094.43640 & 186.22511
\end{tabular}

*** End of Report *** 
<smiles>C=C[C@H](c1ccc(Cl)c(Cl)c1)[C@@]1(C(C)=O)COCC1c1ccc(C(F)(F)F)cc1</smiles>

\section{$(S, S)-3 h f$}

Data File E: \DATA WHM WHM-3-53 2019-69-25 21-24-11\WHM-3-531.D Sample Name: WHM-3-53-M,P-CL-RS

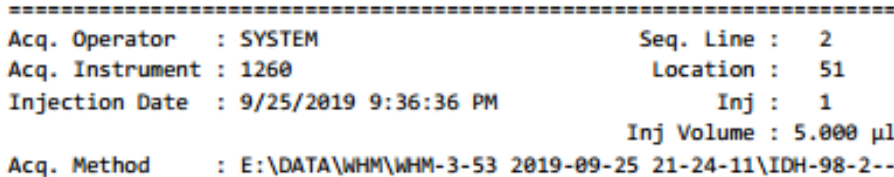

Acq. Method : E: \DATA WHMYWHM-3-53 2019-69-25 21-24-11 \IDH-98-2--1ML-20MIN-5UL.M Last changed : 9/25/2019 9:24:12 PM by SYSTEM

Analysis Method : E: \DATA WHMYWHM-3-53 2019-09-25 21-24-11】IDH-98-2--1ML-20MIN-5UL.M ( Sequence Method)

Last changed : 10/23/2019 4:39:41 PM by SYSTEM (modified after loading)

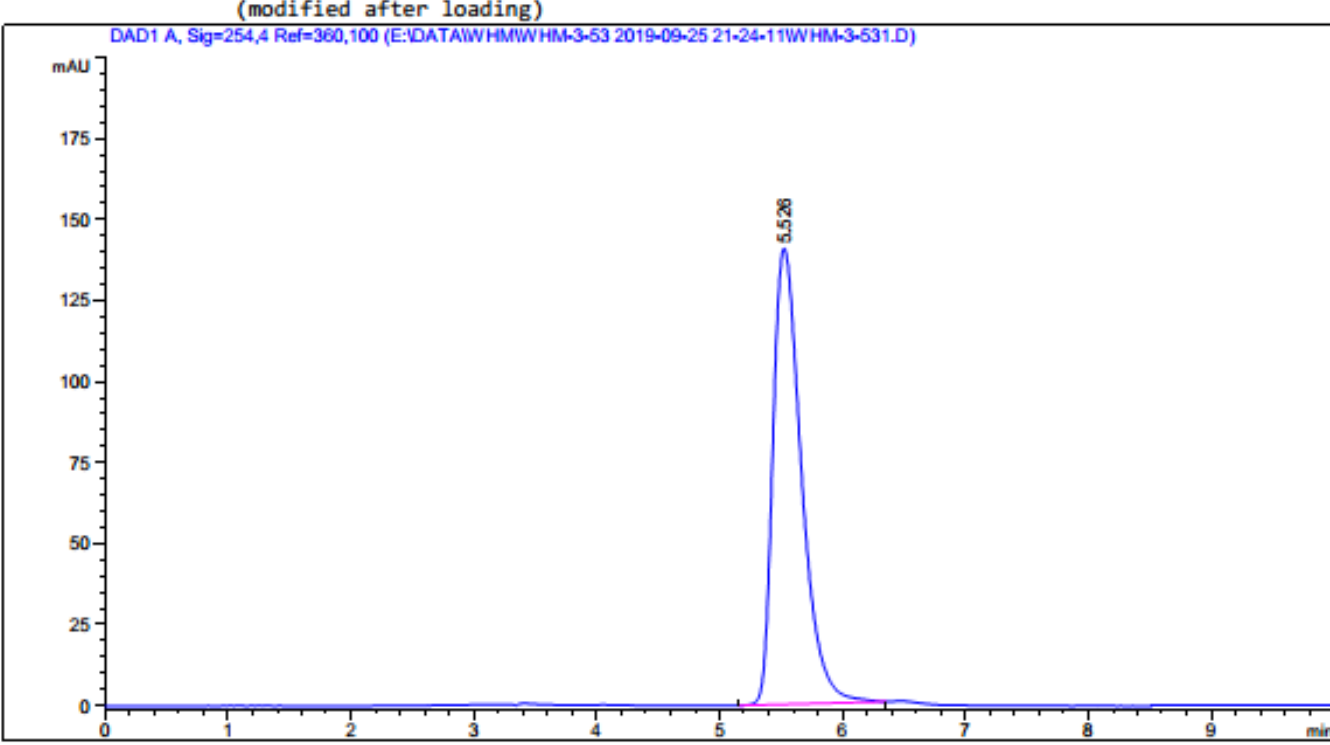

Area Percent Report

\begin{tabular}{|c|c|c|}
\hline Sorted By & : & Signal \\
\hline Multiplier & : & 1.0000 \\
\hline Dilution & : & 1.0000 \\
\hline
\end{tabular}

Use Multiplier \& Dilution Factor with ISTDs

Signal 1: DAD1 A, Sig=254,4 Ref=360, 100

\begin{tabular}{|c|c|c|c|c|c|}
\hline $\begin{array}{c}\text { Peak } \\
\#\end{array}$ & $\begin{array}{l}\text { RetTime Type } \\
\text { [min] }\end{array}$ & $\begin{array}{l}\text { Width } \\
\text { [min] }\end{array}$ & $\begin{array}{c}\text { Area } \\
{\left[\mathrm{mAU}^{*} \mathrm{~s}\right]}\end{array}$ & $\begin{array}{l}\text { Height } \\
\text { [mAU] }\end{array}$ & $\begin{array}{c}\text { Area } \\
\text { \% }\end{array}$ \\
\hline
\end{tabular}

$\begin{array}{lllllll}1 & 5.526 & \text { BB } & 0.2479 & 2265.18286 & 140.65492 & 100.0090\end{array}$

Totals : $\quad 2265.18286 \quad 140.65402$ 
<smiles>C=CC(c1cccc(Cl)c1)[C@]1(C)COC(=O)C1(C)C(C)(C)C</smiles>

Data file E: \DATA WHM WHM-3-46-54-RSSRRAC 2019-09-26 14-49-93\WHM-3-47-54-RAC4.D Sample Name: WHM-3-50-P-CL-RAC

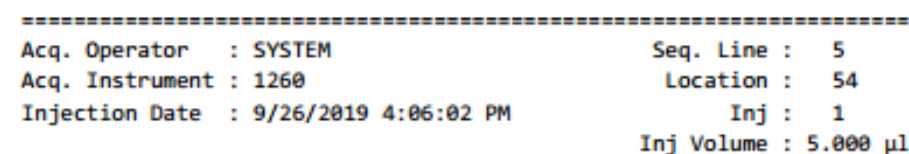

Acq. Method : E: \DATAVWHMYWHM-3-46-54-RSSRRAC 2019-09-26 14-49-03\IDH-98-2--1ML-20MIN-5UL

Last changed : 9/26/2019 2:49:03 PM by SYSTEM

Analysis Method : E: \DATA WHMThHM-3-46-54-RSSRRAC 2019-09-26 14-49-03\IDH-98-2--1ML-20MIN-5UL .M (Sequence Method)

Last changed : 10/23/2019 8:01:33 PM by SYSTEM

(modified after loading)

Additional Info: Peak(s) manually integrated

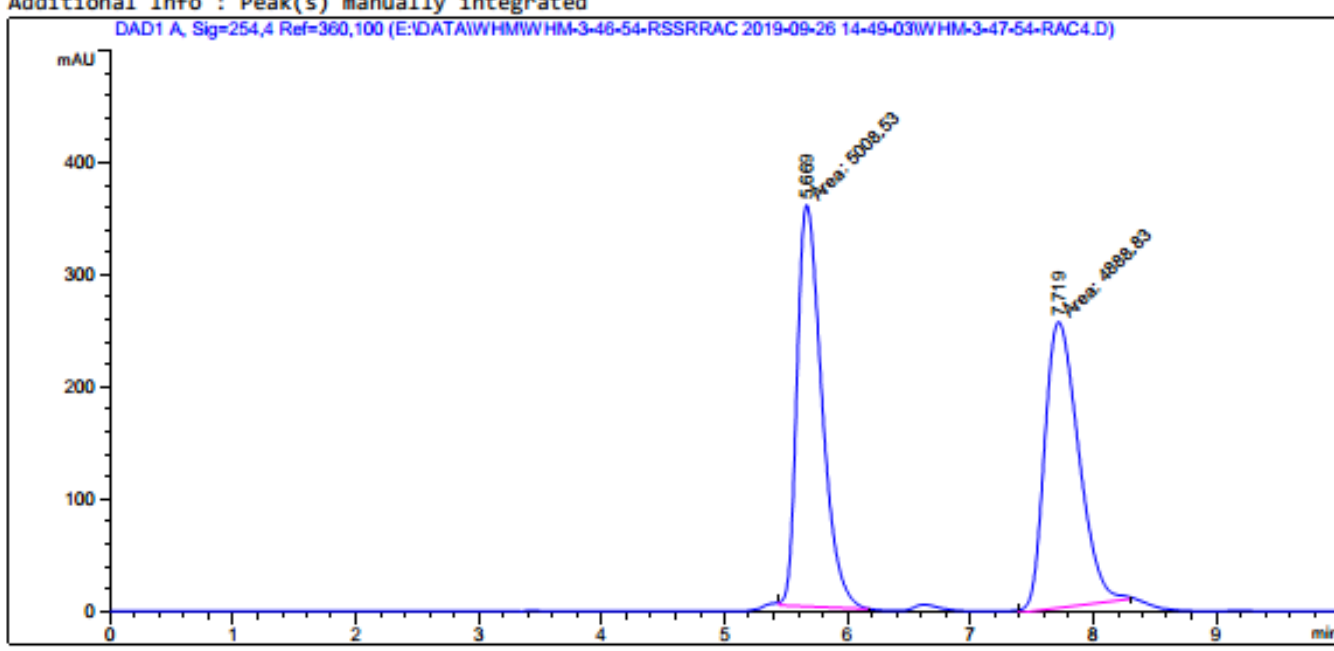

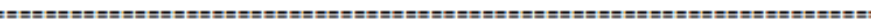

Area Percent Report

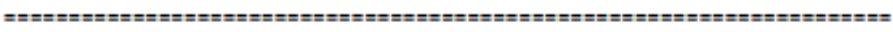

$\begin{array}{lcc}\text { Sorted By } & : & \text { Signal } \\ \text { Multiplier } & : & 1.0000 \\ \text { Dilution } & : & 1.0000\end{array}$

Do not use Multiplier \& Dilution Factor with ISTDs

Signal 1: DAD1 A, Sig $=254,4$ Ref $=360,100$

\begin{tabular}{cccccc}
$\begin{array}{l}\text { Peak RetTime Type } \\
\text { [min] }\end{array}$ & $\begin{array}{c}\text { Width } \\
\text { [min] }\end{array}$ & $\begin{array}{c}\text { Area } \\
\text { [mAU*s] }\end{array}$ & $\begin{array}{l}\text { Height } \\
\text { [mAU] }\end{array}$ & $\begin{array}{c}\text { Area } \\
\%\end{array}$ \\
\hline 1 & $5.669 \mathrm{MM}$ & 0.2334 & 5008.53418 & 357.72272 & 50.6047 \\
2 & $7.719 \mathrm{MM}$ & 0.3200 & 4888.83105 & 254.61388 & 49.3953 \\
& & & & & \\
Totals : & & 9897.36523 & 612.33659
\end{tabular}

*** End of Report *** 
<smiles>C=C[C@H](c1cccc(Cl)c1)[C@@]1(C)COC(c2ccc(C(F)(F)F)cc2)=N[C@H]1C(C)=O</smiles>

\section{$(S, S)$-3hg}

Data file E: \DATA WHM WHM-3-50-47 2019-09-24 99-15-56\WHM-3-50-2.D Sample Name: WHM-3-50-M-CL-RS
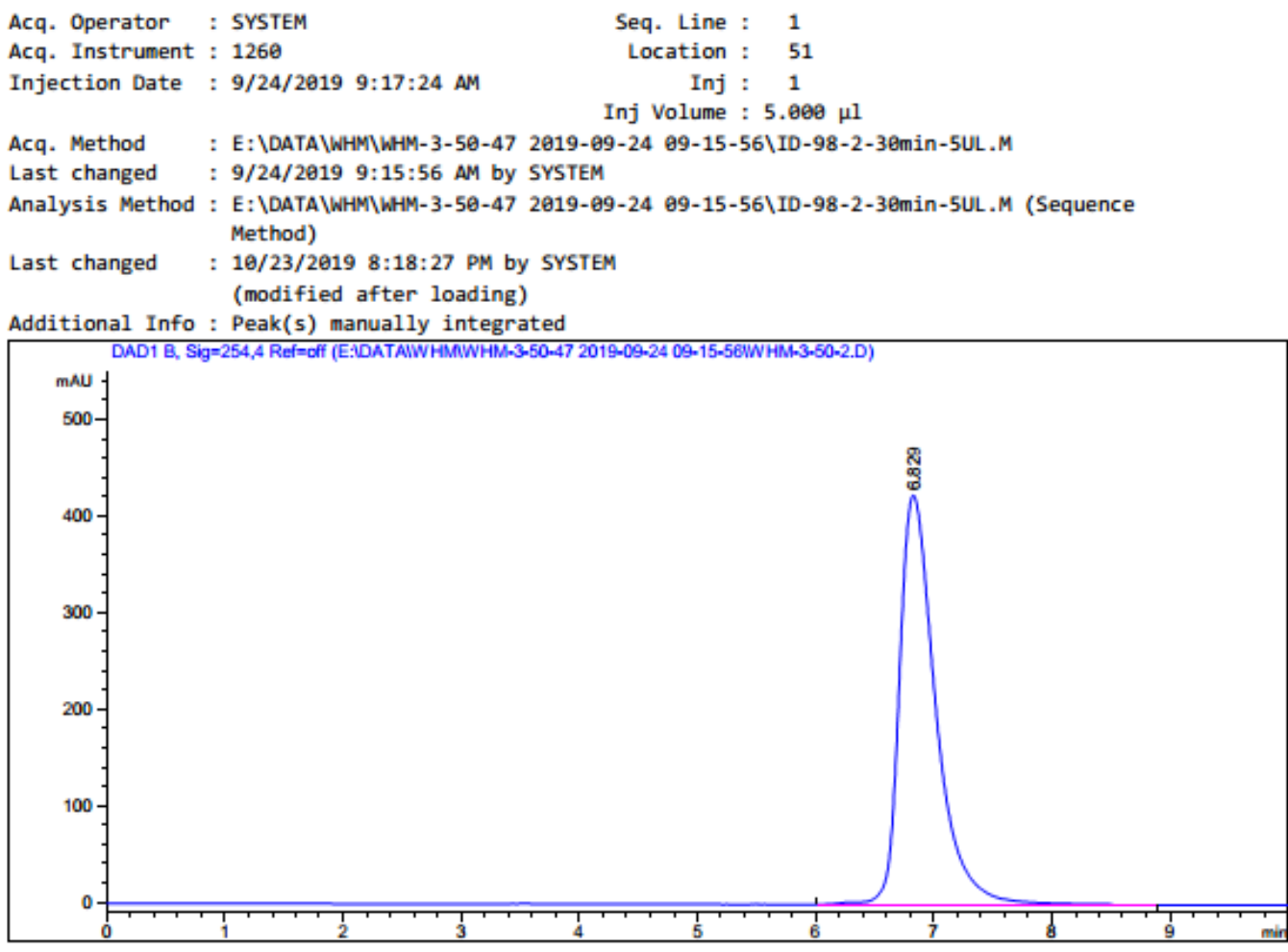

Area Percent Report

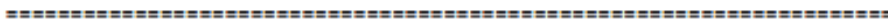

Sorted By : Signal

Multiplier : $\quad 1.0000$

Dilution : 1.0000

Do not use Multiplier \& Dilution Factor with ISTDs

Signal 1: DAD1 B, Sig $=254,4$ Ref $=$ off

\begin{tabular}{|c|c|c|c|c|c|}
\hline $\begin{array}{c}\text { Peak } \\
\#\end{array}$ & $\begin{array}{l}\text { RetTime Type } \\
\text { [min] }\end{array}$ & $\begin{array}{l}\text { Width } \\
\text { [min] }\end{array}$ & $\begin{array}{c}\text { Area } \\
{\left[\mathrm{mAU}^{*} \mathrm{~s}\right]}\end{array}$ & $\begin{array}{l}\text { Height } \\
\text { [mAU] }\end{array}$ & $\begin{array}{c}\text { Area } \\
\text { \% }\end{array}$ \\
\hline
\end{tabular}

Totals:

$9955.77051 \quad 423.66367$

*** End of Report *** 
<smiles>C=CC(c1cccc(C)c1)[C@@]1(C(C)=O)COCC1c1ccc(C(F)(F)F)cc1</smiles>

racemic-3hh

Data file E: \DATA WHM WHM-3-46-49-RAC 2019-99-27 99-36-40\WHM-3-46-49-RAC2.D Sample Name: WHM-3-49-M-ME-RAC

\begin{tabular}{|c|c|c|c|}
\hline $\begin{array}{l}\text { Acq. Operator } \\
\text { Acq. Instrument }\end{array}$ & : SYSTEM & Seq. Line : & 3 \\
\hline Injection Date & : 9/27/2019 10:10:32 AM & Inj & 1 \\
\hline
\end{tabular}

Acq. Method : E: \DATA \WHM\WHM-3-46-49-RAC 2019-99-27 99-36-40\IDH-98-2--1ML-20MIN-5UL.M Last changed : 9/27/2019 9:36:40 AM by SYSTEM

Analysis Method: E: \DATA WWHMWHM-3-46-49-RAC 2019-99-27 99-36-40\IDH-98-2--1ML-20MIN-5UL.M ( Sequence Method)

Last changed : 10/23/2019 7:46:日9 PM by SYSTEM

(modified after loading)

Additional Info: Peak(s) manually integrated

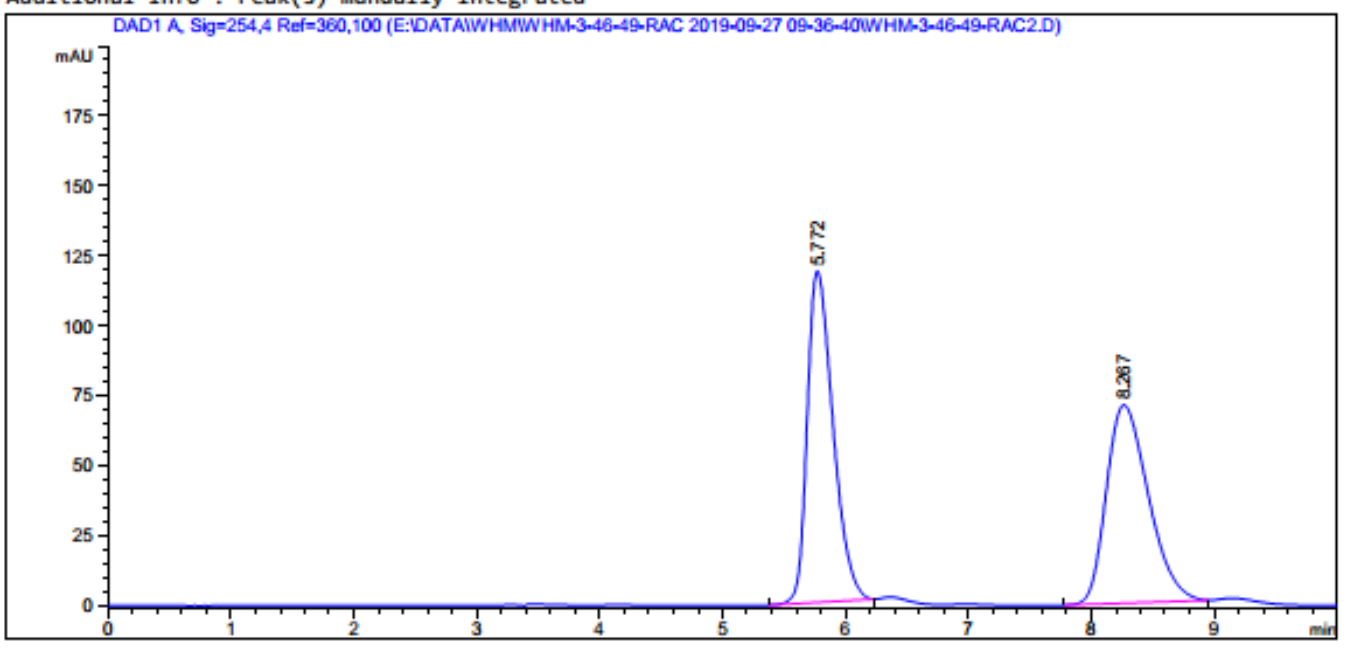

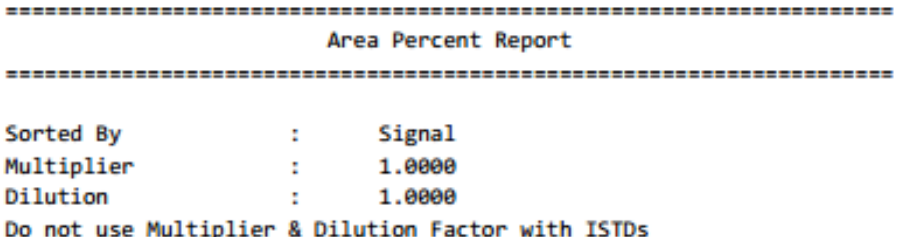

\begin{tabular}{|c|c|c|c|c|c|}
\hline $\begin{array}{c}\text { Peak } \\
\#\end{array}$ & $\begin{array}{l}\text { RetTime Type } \\
\text { [min] }\end{array}$ & $\begin{array}{l}\text { Width } \\
\text { [min] }\end{array}$ & $\begin{array}{c}\text { Area } \\
{\left[\mathrm{mAU}^{*} \mathrm{~s}\right]}\end{array}$ & $\begin{array}{l}\text { Height } \\
\text { [mAU] }\end{array}$ & $\begin{array}{c}\text { Area } \\
\text { \% }\end{array}$ \\
\hline- & & & 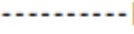 & & - \\
\hline 1 & 5.772 BB & 0.2270 & 1724.78528 & 118.38918 & 50.6386 \\
\hline 2 & $8.267 \mathrm{BB}$ & 0.3701 & 1681.28601 & 70.79421 & 49.3614 \\
\hline
\end{tabular}

Totals : $\quad 3406.07129 \quad 189.18340$

*** End of Report *** 
<smiles>C=CC(c1cccc(C)c1)[C@@]1(C(C)=O)COCC1c1ccc(C(F)(F)F)cc1</smiles>

\section{$(S, S)-3 h h$}

Data File E: \DATA WHMWWHM-3-49-M-ME-RSSR 2019-89-26 21-16-41 \WHM-3-49-RSSR1.D Sample Name: WHM-3-49-M-ME-RS

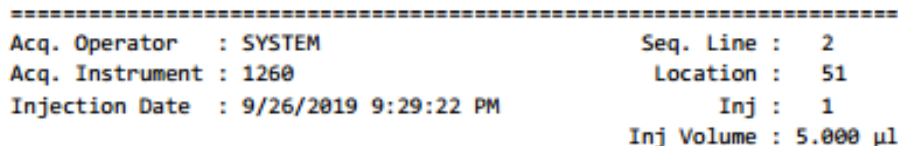

Acq. Method : E: \DATAWWHMWHM-3-49-M-ME-RSSR 2019-99-26 21-16-41\IDH-98-2--1ML-20MIN-5UL.

Last changed : 9/26/2019 9:16:42 PM by SYSTEM

Analysis Method : E: (DATAWWHMWHM-3-49-M-ME-RSSR 2019-09-26 21-16-41\IDH-98-2--1ML-20MIN-5UL. $M$ (Sequence Method)

Last changed : 10/23/2019 8:16:11 PM by SYSTEM (modified after loading)

Additional Info: Peak(s) manually integrated

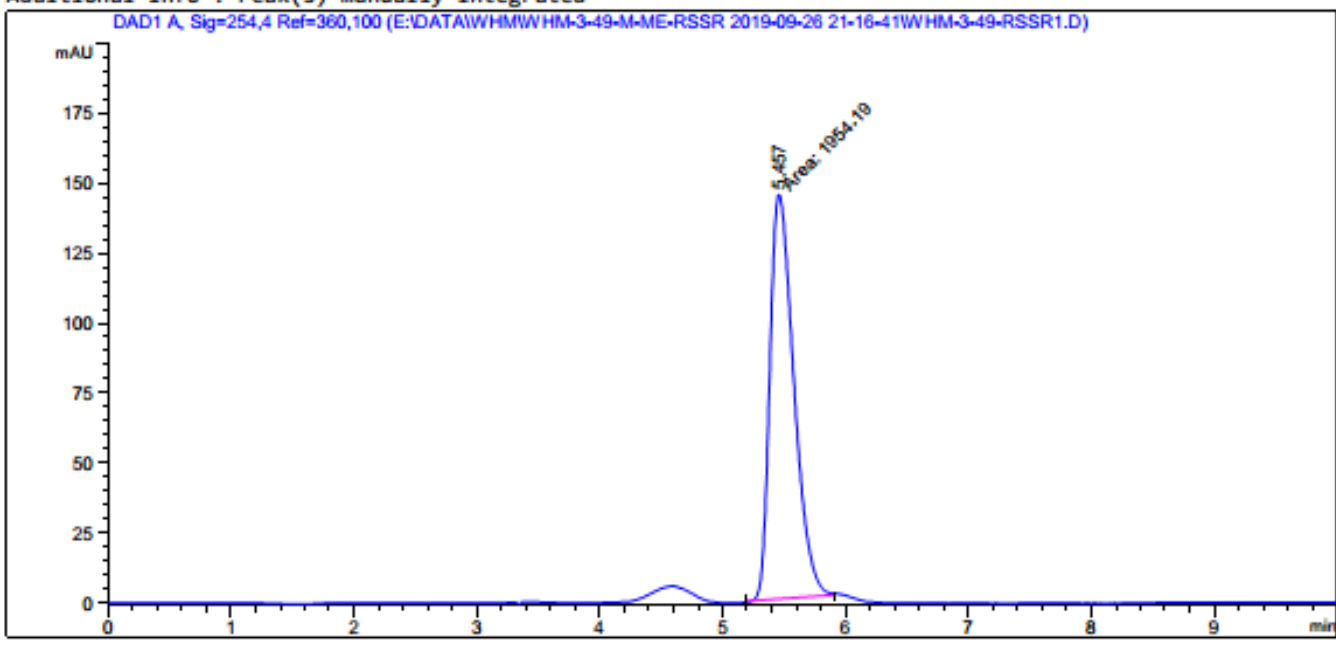

Area Percent Report

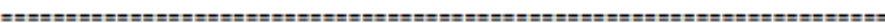

$\begin{array}{lcc}\text { Sorted By } & : & \text { Signal } \\ \text { Multiplier } & : & 1.0000 \\ \text { Dilution } & : & 1.000 \ominus \\ \text { Do not use Multiplier } & \& & \text { Dilution Factor with ISTDs }\end{array}$

Signal 1: DAD1 A, Sig $=254,4$ Ref $=360,100$

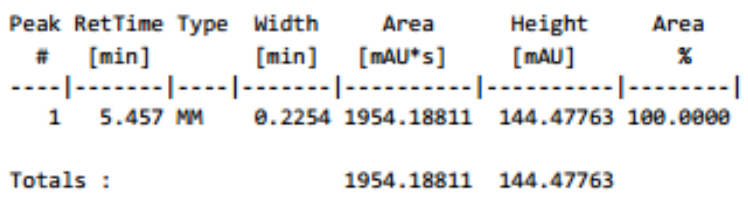

*** End of Report *** 
<smiles>C=CC(c1cccc(OC)c1)[C@]1(C(C)=O)COC(c2ccc(C(F)(F)F)cc2)N1</smiles>

Data file E: \DATA WHM WHM-3-46-54-RSSRRAC 2019-09-26 14-49-03\WHM-3-47-54-RAC5.D Sample Name: WHM-3-51-M-OME-RAC

\begin{tabular}{|c|c|c|c|}
\hline Acq. Operator & : SYSTEM & Seq. Line : & 6 \\
\hline Acq. Instrument & : 1260 & Location : & 55 \\
\hline Injection Date & : 9/26/2019 4:27:33 PM & $\begin{array}{r}\text { Inj : } \\
\text { Inj Volume : }\end{array}$ & 1 \\
\hline
\end{tabular}

Acq. Method : E: \DATAVWHMYWHM-3-46-54-RSSRRAC 2019-09-26 14-49-03\IDH-98-2--1ML-20MIN-5UL

Last changed : 9/26/2019 2:49:03 PM by SYSTEM

Analysis Method : E: \DATA WHMThHM-3-46-54-RSSRRAC 2019-09-26 14-49-03\IDH-98-2--1ML-20MIN-5UL .M (Sequence Method)

Last changed : 10/23/2019 8:03:07 PM by SYSTEM

(modified after loading)

Additional Info: Peak(s) manually integrated

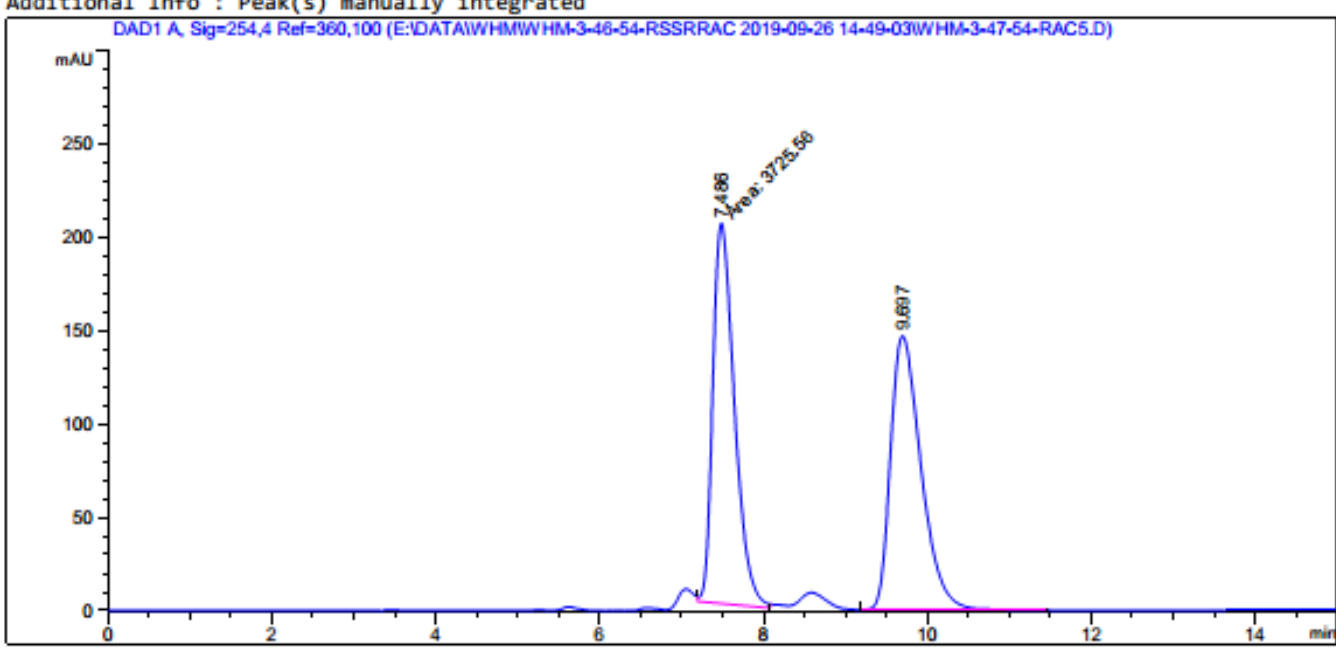

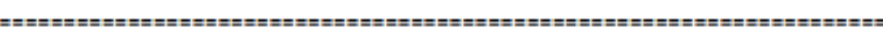

Area Percent Report

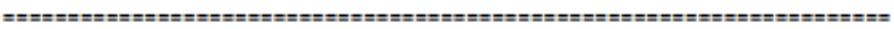

$\begin{array}{llc}\text { Sorted By } & : & \text { Signal } \\ \text { Multiplier } & : & 1.000 \theta \\ \text { Dilution } & : & 1.0000\end{array}$

Do not use Multiplier \& Dilution Factor with ISTDs

Signal 1: DAD1 A, Sig $=254,4$ Ref $=360,100$

\begin{tabular}{llllll}
$\begin{array}{l}\text { Peak RetTime Type } \\
\text { [min] }\end{array}$ & $\begin{array}{c}\text { Width } \\
\text { [min] }\end{array}$ & $\begin{array}{c}\text { Area } \\
\text { [mAU*s] }\end{array}$ & $\begin{array}{c}\text { Height } \\
\text { [mAU] }\end{array}$ & $\begin{array}{c}\text { Area } \\
\%\end{array}$ \\
\hline 1 & $7.486 \mathrm{MM}$ & 0.3053 & 3725.55591 & 203.39958 & 49.4461 \\
2 & $9.697 \mathrm{BB}$ & 0.4030 & 3809.02197 & 146.68326 & 50.5539 \\
& & & & &
\end{tabular}

*** End of Report *** 
<smiles>C=CC(c1cccc(OC)c1)C1(C(C)=O)COC(c2ccc(C(F)(F)F)cc2)=N1</smiles>

$(S, S)-3 h i$

Data file E: \DATA WHM\WHM-3-51-52 2019-09-24 17-28-59\WHM-3-51-52.D Sample Name: WHM-3-51-M-OME-RS

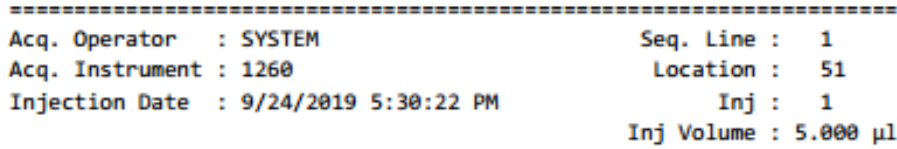

Acq. Method : E: \DATAWWHMWWHM-3-51-52 2019-09-24 17-28-59\ID-98-2-38min-5UL.M

Last changed : 9/24/2019 5:28:59 PM by SYSTEM

Analysis Method : E: \DATA WWHMWHM-3-51-52 2019-99-24 17-28-59\ID-98-2-3emin-5UL.M (Sequence Method)

Last changed : 10/23/2019 $8: 21: 53$ PM by SYSTEM

(modified after loading)

Additional Info: Peak(s) manually integrated

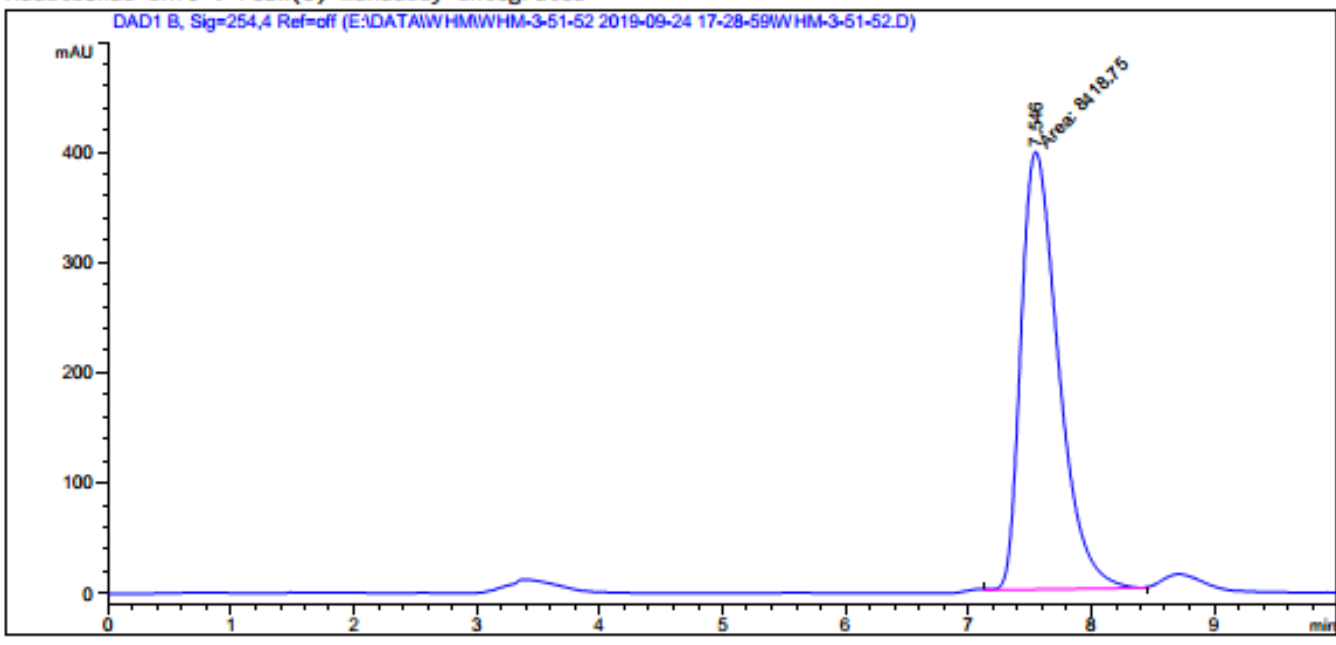

Area Percent Report

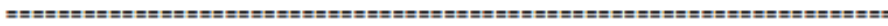

Sorted By : : Signal

Multiplier : : 1.0000

Dilution : 1.0000

Do not use Multiplier \& Dilution Factor with ISTDs

Signal 1: DAD1 B, Sig $=254,4$ Ref $=$ off

\begin{tabular}{|c|c|c|c|c|c|}
\hline $\begin{array}{c}\text { Peak } \\
\#\end{array}$ & $\begin{array}{l}\text { RetTime Type } \\
\text { [min] }\end{array}$ & $\begin{array}{l}\text { Width } \\
\text { [min] }\end{array}$ & $\begin{array}{c}\text { Area } \\
{\left[\mathrm{mAU}^{*} \mathrm{~s}\right]}\end{array}$ & $\begin{array}{l}\text { Height } \\
\text { [mAU] }\end{array}$ & $\begin{array}{c}\text { Area } \\
\text { \% }\end{array}$ \\
\hline
\end{tabular}

Totals

$8418.74962 \quad 396.88333$

*** End of Report *** 


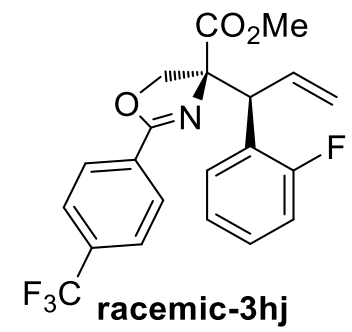

Data file E: (DATA WHM WHM-3-75-0-F-RAC-3 2019-10-16 08-57-34 WWHM-3-75-0-F-RAC1.D Sample Name: WHM-3-75-0-F-RAC

\begin{tabular}{|c|c|c|c|}
\hline Acq. Operator & : SYSTEM & Seq. Line : & 2 \\
\hline Acq. Instrument : & : 1260 & Location : & 11 \\
\hline Injection Date & : 10/16/2019 9:09:56 AM & $\begin{array}{r}\text { Inj : } \\
\text { Inj Volume : }\end{array}$ & $\begin{array}{c}1 \\
.00\end{array}$ \\
\hline
\end{tabular}

Acq. Method : E: \DATAWWHMWHM-3-75-0-F-RAC-3 2019-10-16 98-57-34\IDH-98-2--1ML-20MIN-5UL.

Last changed : 10/16/2019 8:57:34 AM by SYSTEM

Analysis Method : E: \DATAWWHM WHM-3-75-0-F-RAC-3 2019-10-16 08-57-34\IDH-98-2--1ML-20MIN-5UL. $M$ (Sequence Method)

Last changed : 10/23/2019 8:39:33 PM by SYSTEM (modified after loading)

Additional Info : Peak(s) manually integrated
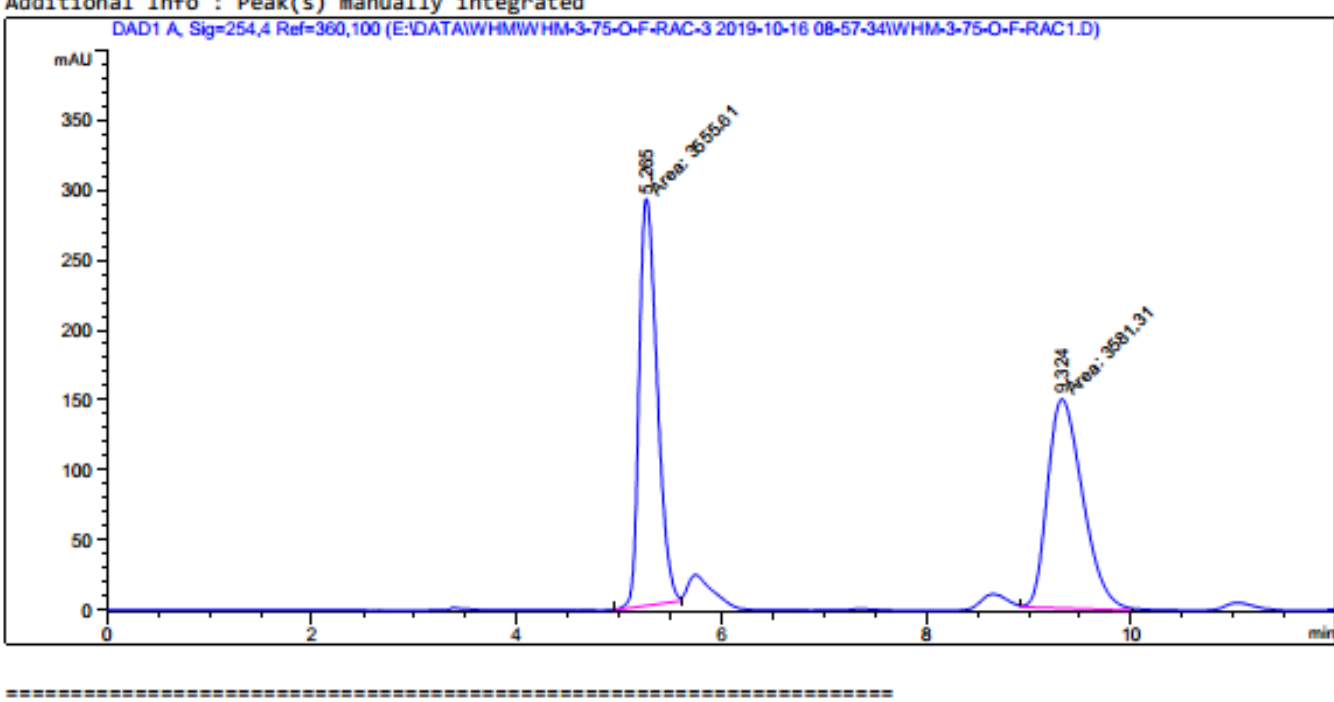

Area Percent Report

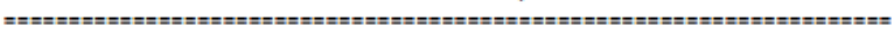

$\begin{array}{lcc}\text { Sorted By } & : & \text { Signal } \\ \text { Multiplier } & : & 1.0000 \\ \text { Dilution } & : & 1.0000\end{array}$

Signal 1: DAD1 A, Sig $=254,4$ Ref $=360,100$

\begin{tabular}{llllll}
$\begin{array}{l}\text { Peak RetTime Type } \\
\text { [min] }\end{array}$ & $\begin{array}{c}\text { Width } \\
\text { [min] }\end{array}$ & $\begin{array}{c}\text { Area } \\
\text { [mAU*s] }\end{array}$ & $\begin{array}{l}\text { Height } \\
\text { [mAU] }\end{array}$ & $\begin{array}{c}\text { Area } \\
\%\end{array}$ \\
\hline 1 & $5.265 \mathrm{MM}$ & 0.2035 & 3555.60547 & 291.13937 & 49.8199 \\
2 & $9.324 \mathrm{MM}$ & 0.3991 & 3581.31274 & 149.57440 & 50.1801 \\
& & & & & \\
Totals : & & 7136.91821 & 440.71378
\end{tabular}

*** End of Report *** 
<smiles>C=CC(c1ccccc1F)[C@@]1(C(C)=O)COC(=O)N1C(=O)c1ccc(C(F)(F)F)cc1</smiles>

Data File E: \DATA WWHM\WHM-3-75-0-F-3 2019-10-15 17-22-52 WWHM-3-75-0-F-31.D Sample Name: WHM-3-75-0-F-RS

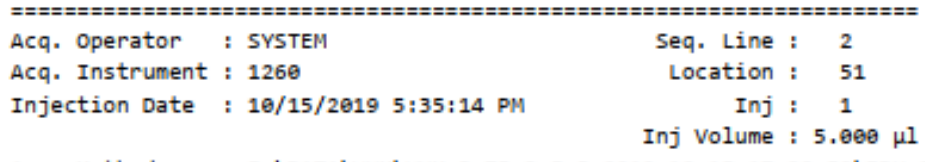

Acq. Method : E: \DATA WWHM\WHM-3-75-0-F-3 2019-10-15 17-22-52\IDH-98-2--1ML-20MIN-5UL. M Last changed : 10/15/2019 5:22:52 PM by SYSTEM

Analys is Method: E: \DATA WHM\WHM-3-75-0-F-3 2019-10-15 17-22-52\IDH-98-2--1ML-20MIN-5UL. M ( Sequence Method)

Last changed : 4/30/2020 10:33:41 PM by SYSTEM (modified after loading)

Additional Info: Peak(s) manually integrated

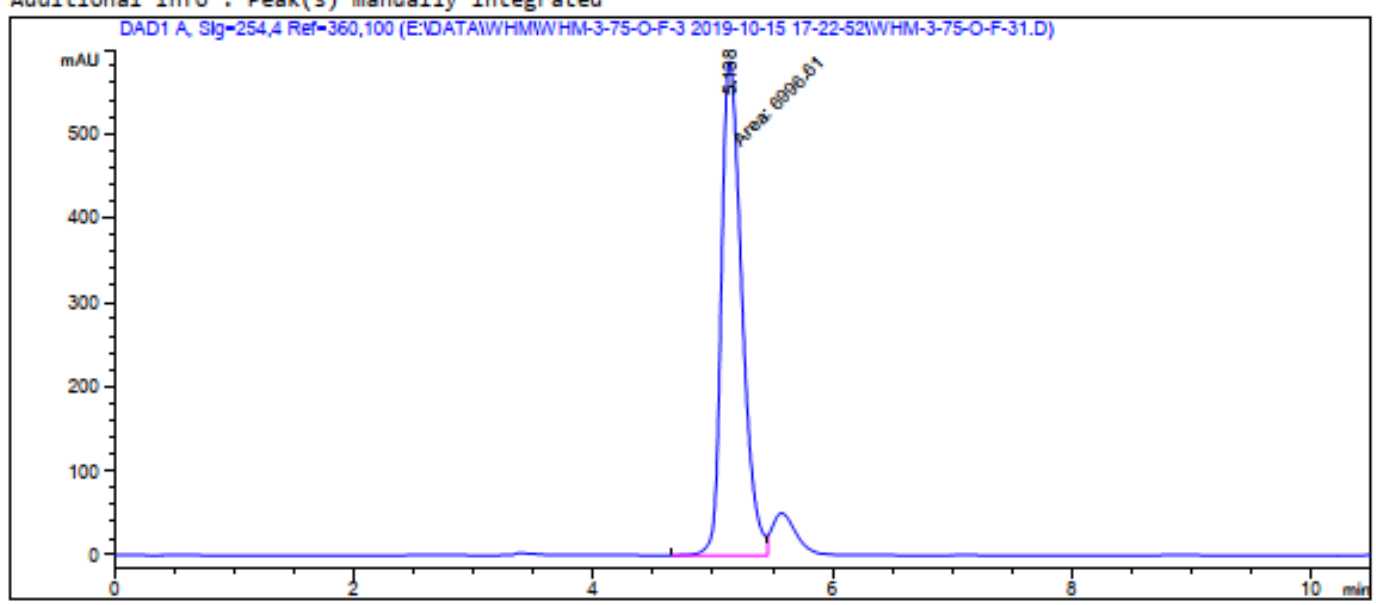

\section{Area Percent Report}

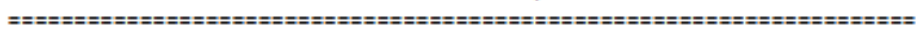

$\begin{array}{lll}\text { Sorted By } & : & \text { Signal } \\ \text { Multiplier } & : & 1.0000 \\ \text { Dilution } & : & 1.0000\end{array}$

Dilution 1.0000

Do not use Multiplier \& Dilution Factor with ISTDs

Signal 1: DAD1 A, Sig $=254,4$ Ref $=360,100$

\begin{tabular}{|c|c|c|c|c|c|}
\hline $\begin{array}{c}\text { Peak } \\
\#\end{array}$ & $\begin{array}{l}\text { RetTime Type } \\
\text { [min] }\end{array}$ & $\begin{array}{l}\text { Width } \\
\text { [min] }\end{array}$ & $\begin{array}{c}\text { Area } \\
{\left[\mathrm{mAu}^{+} \mathrm{s}\right]}\end{array}$ & $\begin{array}{l}\text { Height } \\
\text { [mAU] }\end{array}$ & $\begin{array}{c}\text { Area } \\
\%\end{array}$ \\
\hline 1 & $5.138 \mathrm{MF}$ & 0.1988 & 6996.60889 & 586.54407 & 100.0000 \\
\hline ota] & : & & 6996.60889 & 586.54407 & \\
\hline
\end{tabular}


<smiles>C=CC(c1ccc2ccccc2c1)C(C)(COC(=O)c1ccc(C(F)(F)F)cc1)C(C)=O</smiles>

racemic-3hk

Data File E: \DATA WHM \WHM-3-50-47 2019-99-24 99-15-56\WHM-3-50-22.D Sample Name: WHM-3-47-2-NA-RAC

\begin{tabular}{|c|c|c|c|}
\hline Acq. Operator & : SYSTEM & Seq. Line & 3 \\
\hline Acq. Instrument & : 1260 & Location & 53 \\
\hline Injection Date & : 9/24/2019 10:20:22 AM & $\begin{array}{r}\text { Inj } \\
\text { Inj Volume }\end{array}$ & $\begin{array}{lc}: & 1 \\
: & 5.000 \mu 1\end{array}$ \\
\hline
\end{tabular}

Acq. Method : E: \DATA :WHM\WHM-3-50-47 2019-09-24 99-15-56\ID-98-2-30min-5UL.M

Last changed : 9/24/2019 9:15:56 AM by SYSTEM

Analysis Method : E: \DATA WWHM\WHM-3-50-47 2019-99-24 99-15-56\ID-98-2-3emin-5UL.M (Sequence Method)

Last changed : 10/23/2019 7:52:16 PM by SYSTEM

(modified after loading)

Additional Info : Peak(s) manually integrated

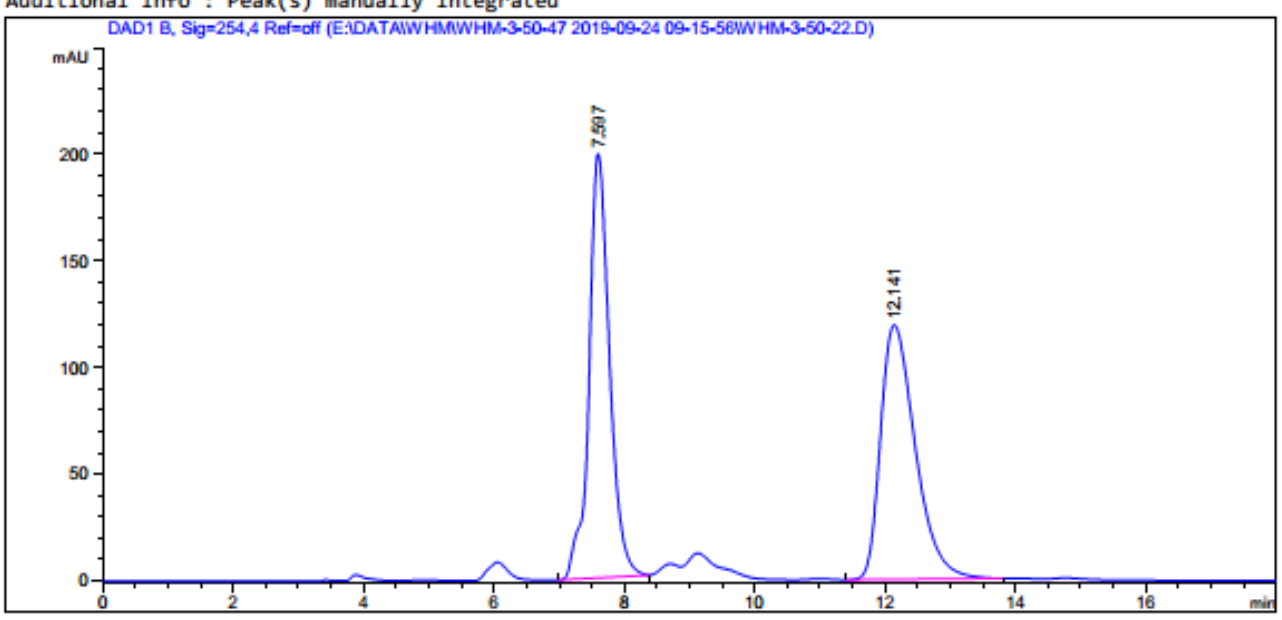

Area Percent Report

$\begin{array}{lll}\text { Sorted By } & : & \text { Signal } \\ \text { Multiplier } & : & 1.0000 \\ \text { Dilution } & : & 1.0000\end{array}$

1.0000

Do not use Multiplier \& Dilution Factor with ISTDs

Signal 1: DAD1 B, Sig=254,4 Ref=off

\begin{tabular}{|c|c|c|c|c|c|}
\hline $\begin{array}{c}\text { Peak } \\
\#\end{array}$ & $\begin{array}{l}\text { RetTime Type } \\
\text { [min] }\end{array}$ & $\begin{array}{l}\text { Width } \\
\text { [min] }\end{array}$ & $\begin{array}{c}\text { Area } \\
{[\mathrm{maU} * \mathrm{~s}]}\end{array}$ & $\begin{array}{l}\text { Height } \\
\text { [maU] }\end{array}$ & $\begin{array}{c}\text { Area } \\
\text { \% }\end{array}$ \\
\hline$\cdots$ & $|\cdots|$ & ........ & 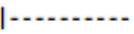 & | & ............ \\
\hline 1 & $7.597 \mathrm{BB}$ & 0.3280 & 4347.61328 & 198.92542 & 49.5797 \\
\hline 2 & $12.141 \mathrm{BB}$ & 0.5653 & 4421.31934 & 119.32416 & 50.4203 \\
\hline
\end{tabular}

Totals: $8768.93262 \quad 318.24957$

*** End of Report *** 
<smiles>C=CC(c1ccc2ccccc2c1)C(C)(CO)NC(=O)c1ccc(C(F)(F)F)cc1</smiles>

$(S, S)-3 h k$

Data File E: \DATA WWHM\WHM-3-46-54-RSSRRAC 2019-99-26 14-49-03\WHM-3-47-54-RAC3.D Sample Name: WHM-3-47-2-NA-RS

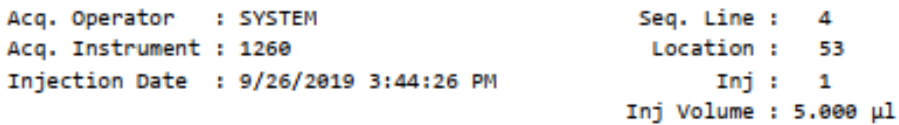

Acq. Method : E: \DATA WWHM WHM-3-46-54-RSSRRAC 2019-09-26 14-49-93\IDH-98-2--1ML-20MIN-5UL .M

Last changed : 9/26/2019 2:49:03 PM by SYSTEM

Analysis Method: E: \DATA WHMM WHM-3-46-54-RSSRRAC 2019-09-26 14-49-93\IDH-98-2--1ML-20MIN-5UL .M (Sequence Method)

Last changed : 4/30/2020 6:02:07 PM by SYSTEM (modified after loading)

Additional Info: Peak(s) manually integrated

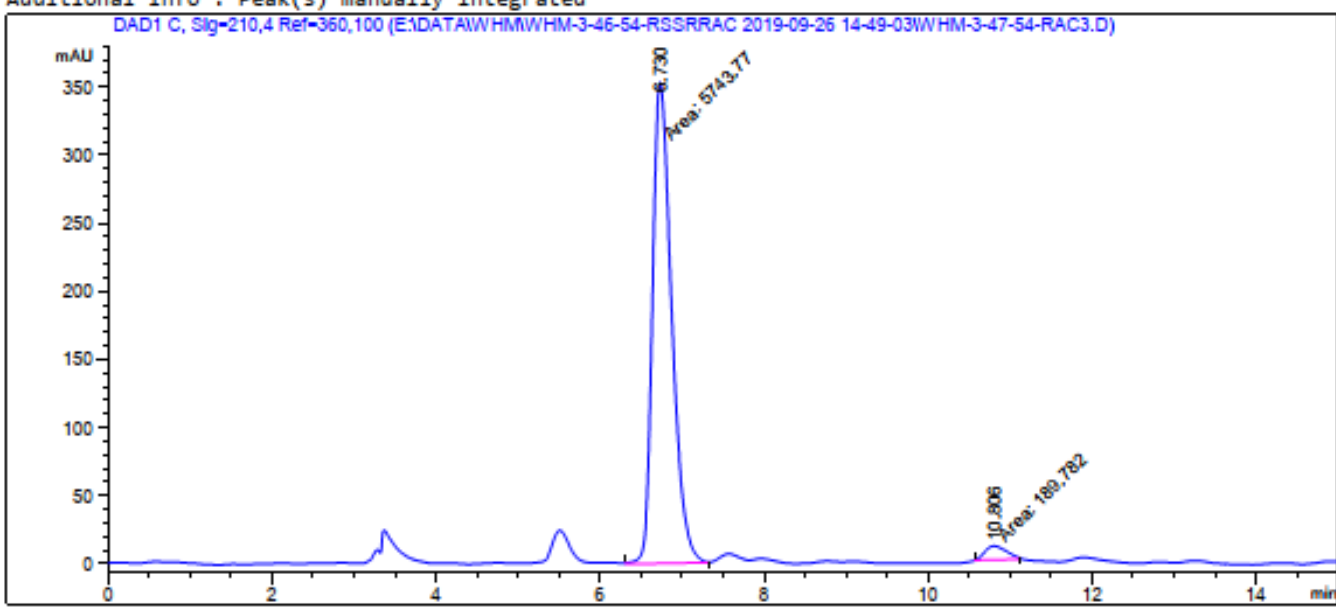

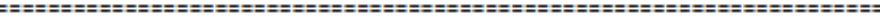

Area Percent Report

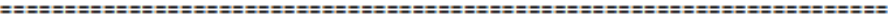

Sorted By : signal

Multiplier : 1.0000

Dilution : 1.0000

Do not use Multiplier \& Dilution Factor with ISTDs

Signal 1: DAD1 C, Sig $=210,4$ Ref $=360,100$

\begin{tabular}{|c|c|c|c|c|c|}
\hline $\begin{array}{c}\text { Peak } \\
\#\end{array}$ & $\begin{array}{l}\text { RetTime Type } \\
\text { [min] }\end{array}$ & $\begin{array}{l}\text { Width } \\
\text { [min] }\end{array}$ & $\begin{array}{c}\text { Area } \\
{\left[\mathrm{mau}^{+} \mathrm{s}\right]}\end{array}$ & $\begin{array}{l}\text { Height } \\
\text { [MAU] }\end{array}$ & $\begin{array}{c}\text { Area } \\
\%\end{array}$ \\
\hline$\ldots$ & $|\ldots \ldots|-\ldots$ & & $\mid-\ldots$. & . & $\ldots \ldots$ \\
\hline 1 & $6.730 \mathrm{~mm}$ & 0.2710 & 5743.77148 & 353.30453 & 96.8016 \\
\hline 2 & $10.806 \mathrm{~mm}$ & 0.3070 & 189.78154 & 10.30281 & 3.1984 \\
\hline ota: & : & & 5933.55302 & 363.60735 & \\
\hline
\end{tabular}

*** End of Report *** 


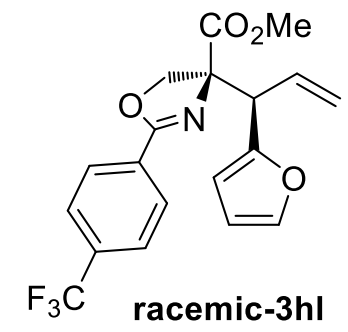

Data File E: \DATA WHM WHM-3-113-BU-PH-BN-SR 2019-11-13 16-48-22\WHM-3-113-BU-PH-BN-SR6.D Sample Name: WHM-3-112-0-RAC

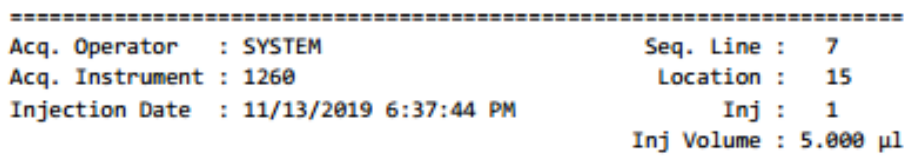

Acq. Method : E: \DATA WWHM WHM-3-113-BU-PH-BN-SR 2019-11-13 16-48-22 \IDH-98-2--1ML-28MINSUL.M

Last changed : 11/13/2019 5:37:16 PM by SYSTEM

Analysis Method : E: \DATAWWHMWHM-3-113-BU-PH-BN-SR 2019-11-13 16-48-22\IDH-98-2--1ML-20MIN5UL.M (Sequence Method)

Last changed : 11/13/2019 9:36:04 PM by SYSTEM (modified after loading)

Additional Info : Peak(s) manually integrated
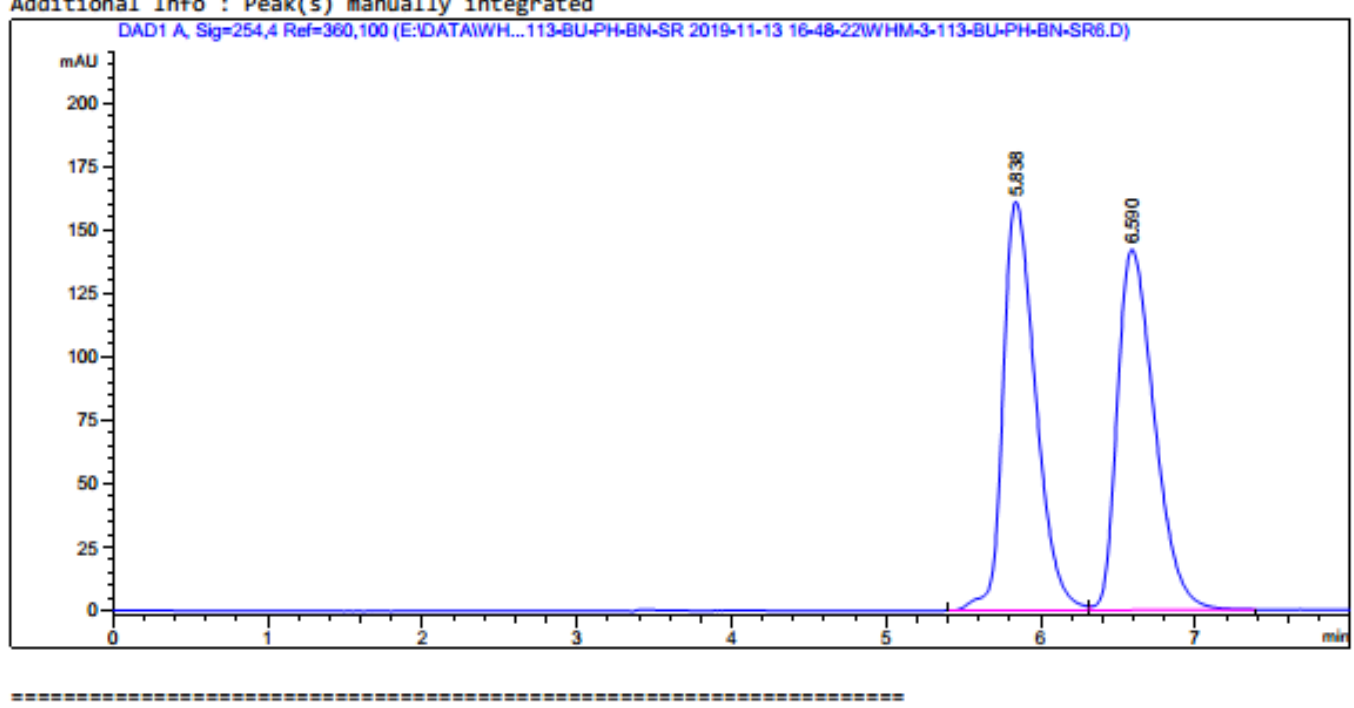

Area Percent Report

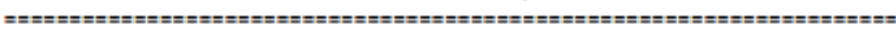

$\begin{array}{lll}\text { Sorted By } & : & \text { Signal } \\ \text { Multiplier } & : & 1.0000 \\ \text { Dilution } & : & 1.0000\end{array}$

Do not use Multiplier \& Dilution Factor with ISTDs

Signal 1: DAD1 A, Sig $=254,4$ Ref $=360,100$

\begin{tabular}{|c|c|c|c|c|c|}
\hline $\begin{array}{c}\text { Peak } \\
\#\end{array}$ & $\begin{array}{l}\text { RetTime Type } \\
\text { [min] }\end{array}$ & $\begin{array}{l}\text { Width } \\
\text { [min] }\end{array}$ & $\begin{array}{c}\text { Area } \\
{\left[\mathrm{mAU}^{*} \mathrm{~s}\right]}\end{array}$ & $\begin{array}{l}\text { Height } \\
\text { [mAU] }\end{array}$ & $\begin{array}{c}\text { Area } \\
\%\end{array}$ \\
\hline$\cdots$ & $|\cdots|$ & 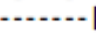 & | & |-..- & - \\
\hline 1 & $5.838 \mathrm{BV}$ & 0.2257 & 2350.43408 & 160.68533 & 49.8630 \\
\hline 2 & $6.590 \mathrm{VB}$ & 0.2585 & 2363.35059 & 141.78075 & 50.1370 \\
\hline Total & 1s: & & 4713.78467 & 302.46608 & \\
\hline
\end{tabular}

*** End of Report *** 


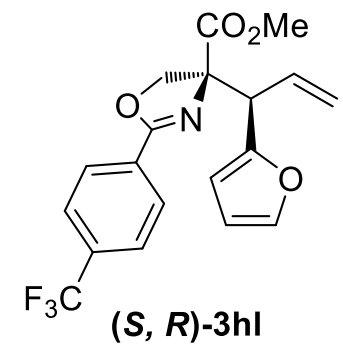

Data File E: \DATA WHM WHM-3-103-111-112 2019-11-13 09-11-00\WHM-3-103-HBW-RAC-111-1125.D Sample Name: WHM-3-112-0-RS

\begin{tabular}{|c|c|c|c|}
\hline $\begin{array}{l}\text { Acq. Operator } \\
\text { Acq. Instrument }\end{array}$ & $\begin{array}{l}: \text { SYSTEM } \\
: 1260\end{array}$ & $\begin{array}{l}\text { Seq. Line : } \\
\text { Location : }\end{array}$ & $\begin{array}{l}6 \\
14\end{array}$ \\
\hline Injection Date & : 11/13/2019 10:33:47 AM & $\begin{array}{r}\text { Inj : } \\
\text { Inj Volume : }\end{array}$ & $\begin{array}{l}1 \\
5 . \theta 00\end{array}$ \\
\hline
\end{tabular}

Acq. Method : E: \DATA WWHMIWHM-3-103-111-112 2019-11-13 99-11-00\IDH-98-2--1ML-20MIN-5UL.M Last changed : 11/13/2019 9:11:00 AM by SYSTEM

Analysis Method : E: \DATA WHMYWHM-3-103-111-112 2019-11-13 09-11-00\IDH-98-2--1ML-20MIN-5UL.M (Sequence Method)

Last changed : 11/13/2019 9:47:33 PM by SYSTEM

(modified after loading)

Additional Info: Peak(s) manually integrated

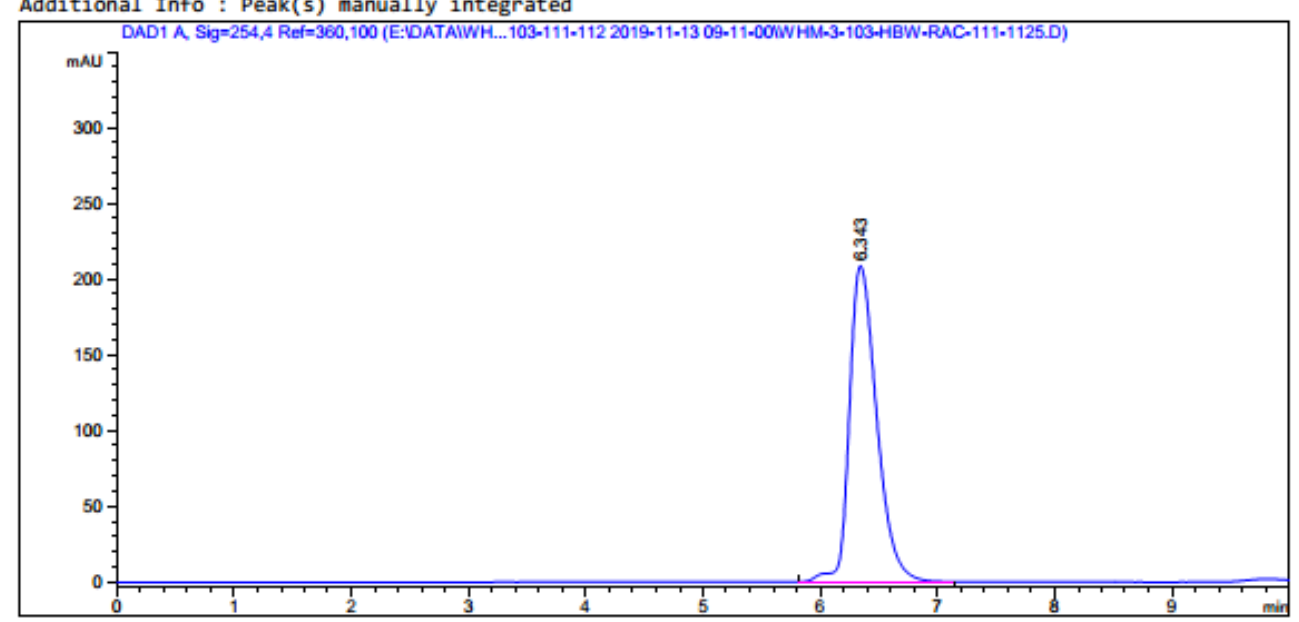

Area Percent Report

$\begin{array}{lll}\text { Sorted By } & : & \text { Signal } \\ \text { Multiplier } & : & 1.0000 \\ \text { Dilution } & : & 1.0000\end{array}$

1.0600

Do not use Multiplier \& Dilution Factor with ISTDs

Signal 1: DAD1 A, Sig $=254,4$ Ref $=360,100$

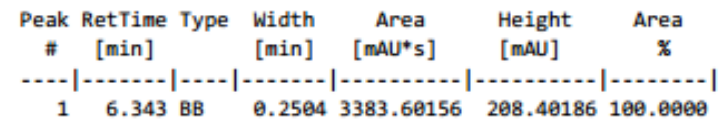

$\begin{array}{llllll}1 & 6.343 \mathrm{BB} & 0.2504 & 3383.69156 & 208.40186 & 100.0009\end{array}$

$\begin{array}{lll}\text { Totals : } & 3383.60156 & 208.40186\end{array}$

*** End of Report *** 


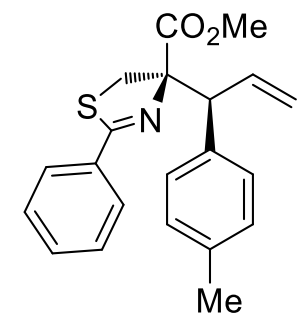

racemic-3ib

Data File E: \DATA WWHYWHM-3-115-P-ME-RSSR 2019-11-14 19-26-24\WHM-3-115-P-ME3.D Sample Name: WHM-3-115-P-ME-RAC

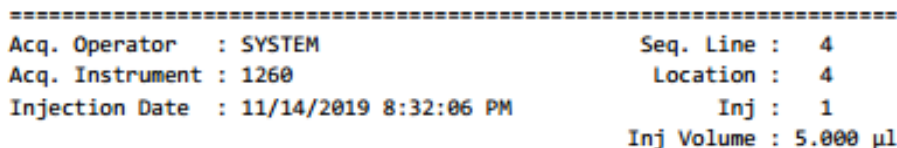

Acq. Method : E: \DATA WHMM WHM-3-115-P-ME-RSSR 2019-11-14 19-26-24\IDH-98-2-5UL-10MIN-1ML.

Last changed : 11/14/2019 $8: 26: 28$ PM by SYSTEM

Analysis Method : E: \DATA WWMM WHM-3-115-P-ME-RSSR 2019-11-14 19-26-24\IDH-98-2-5UL-10MIN-1ML. $M$ (Sequence Method)

Last changed : 11/17/2019 11:18:07 AM by SYSTEM (modified after loading)

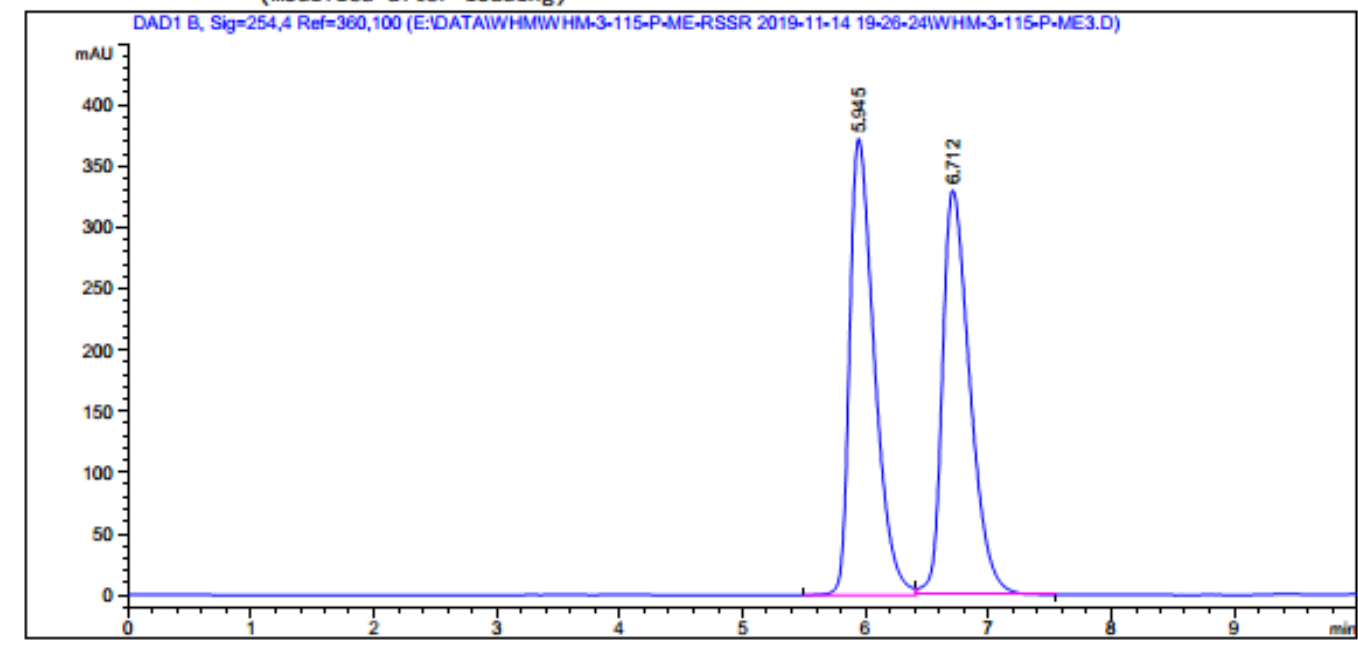

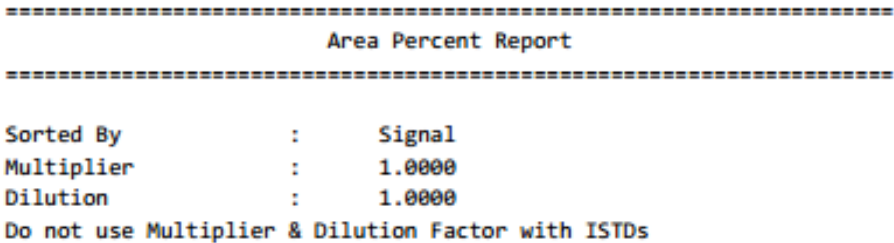

Signal 1: DAD1 B, Sig $=254,4$ Ref $=360,100$

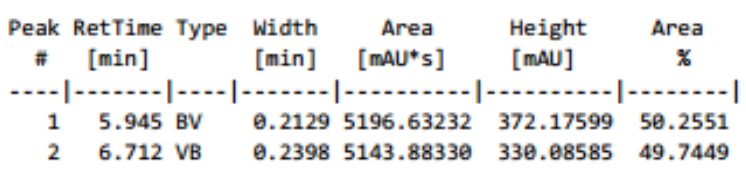

Totals : $\quad 1.03405 \mathrm{e} 4 \quad 702.26184$ 


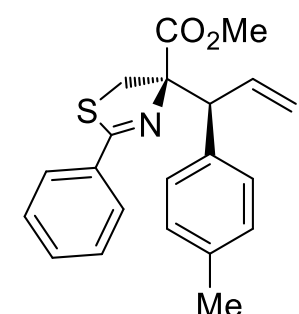

$(R, S)-3 i b$

Data File E: \DATA WHM WHM-3-115-P-ME-RSSR 2019-11-14 19-26-24\WHM-3-115-P-ME.D Sample Name: WHM-3-115-P-ME-RS

\begin{tabular}{|c|c|c|c|}
\hline Acq. Operator & : SYSTEM & Seq. Line : & 1 \\
\hline Acq. Instrument & : 1260 & Location : & 1 \\
\hline Injection Date & : 11/14/2019 7:27:50 PM & $\begin{array}{r}\text { Inj : } \\
\text { Inj Volume : }\end{array}$ & $\begin{array}{cc}: & 1 \\
: & 5.000 \mu \mathrm{l}\end{array}$ \\
\hline
\end{tabular}

Acq. Method : E: \DATA WHMIWHM-3-115-P-ME-RSSR 2019-11-14 19-26-24\IDH-98-2--1ML-20MIN-5UL .M

Last changed : 11/14/2019 7:26:25 PM by SYSTEM

Analysis Method : E: \DATA WHMI WHM-3-115-P-ME-RSSR 2019-11-14 19-26-24\IDH-98-2--1ML-20MIN-5UL .M (Sequence Method)

Last changed : 11/17/2019 11:22:43 AM by SYSTEM

(modified after loading)

Additional Info : Peak(s) manually integrated

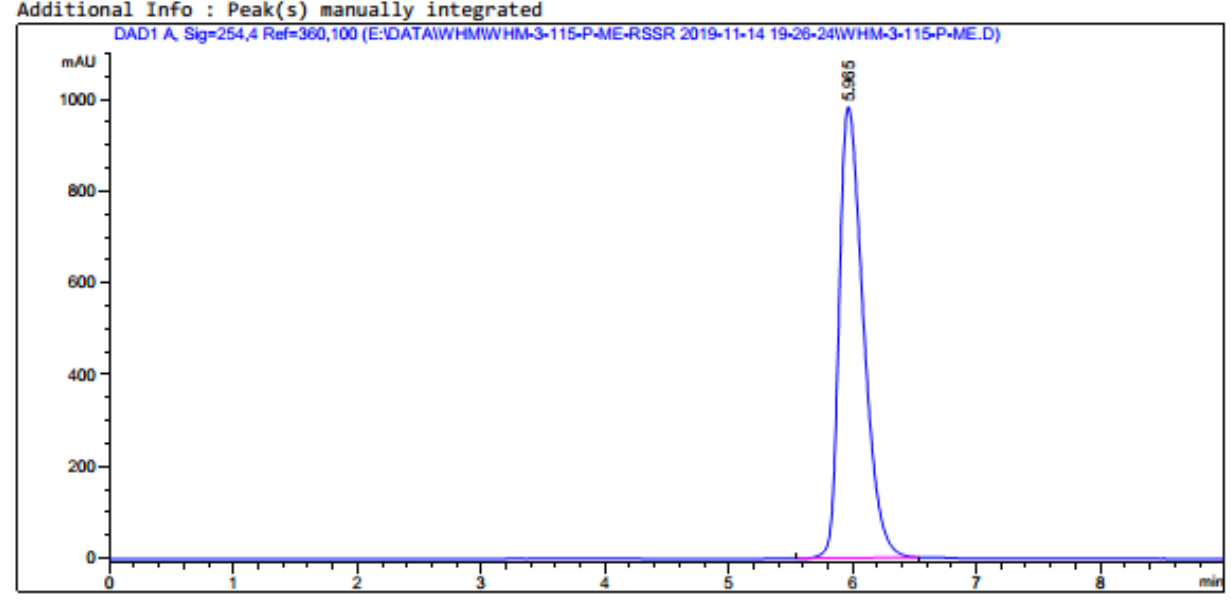

Area Percent Report

\begin{tabular}{|c|c|c|c|c|}
\hline Sorted By & : & \multicolumn{3}{|l|}{ Signal } \\
\hline Multiplier & : & \multicolumn{3}{|l|}{1.0000} \\
\hline Dilution & : & \multicolumn{3}{|l|}{$1 . \ominus 000$} \\
\hline \multicolumn{5}{|c|}{ Do not use Multiplier \& Dilution Factor with ISTDs } \\
\hline \multicolumn{5}{|c|}{ Signal 1: DAD1 A, Sig=254,4 Ref $=360,100$} \\
\hline $\begin{array}{l}\text { Peak RetTime Type } \\
\# \text { [min] }\end{array}$ & $\begin{array}{l}\text { Width } \\
\text { [min] }\end{array}$ & $\begin{array}{c}\text { Area } \\
{\left[\mathrm{mAU}^{*} \mathrm{~s}\right]}\end{array}$ & $\begin{array}{l}\text { Height } \\
\text { [mAU] }\end{array}$ & $\begin{array}{c}\text { Area } \\
\%\end{array}$ \\
\hline$-\cdots|-1-n-1-n|$ & -....... & 1-n.............. & 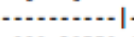 & $|-\cdots+2|$ \\
\hline 15.965 вв & 0.2091 & $1.33127 \mathrm{e} 4$ & 982.32550 & 100.0000 \\
\hline Totals : & & $1.33127 \mathrm{e} 4$ & 982.32550 & \\
\hline
\end{tabular}

*** End of Report *** 


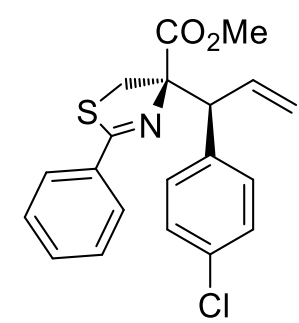

racemic-3ic

Data file E: \DATA WWMYWHM-3-115-P-ME-RSSR 2019-11-14 19-26-24\WHM-3-115-P-ME2.D Sample Name: WHM-3-114-P-CL--RAC

\begin{tabular}{|c|c|c|c|}
\hline Acq. Operator & : SYSTEM & Seq. Line & 3 \\
\hline Acq. Instrument & : 1260 & Location : & 3 \\
\hline Injection Date & : 11/14/2019 8:10:32 PM & $\begin{array}{r}\text { Inj } \\
\text { Inj Volume }\end{array}$ & $\begin{array}{cc}: & 1 \\
: & 5.000 \mu 1\end{array}$ \\
\hline
\end{tabular}

Acq. Method : E: \DATA WHMYWHM-3-115-P-ME-RSSR 2019-11-14 19-26-24\IDH-98-2--1ML-20MIN-5UL .M

Last changed : 11/14/2019 7:26:25 PM by SYSTEM

Analysis Method: E: \DATA WWHM WHM-3-115-P-ME-RSSR 2019-11-14 19-26-24\IDH-98-2--1ML-20MIN-5UL .M (Sequence Method)

Last changed : 11/17/2019 11:25:07 AM by SYSTEM

(modified after loading)

Additional Info: Peak(s) manually integrated

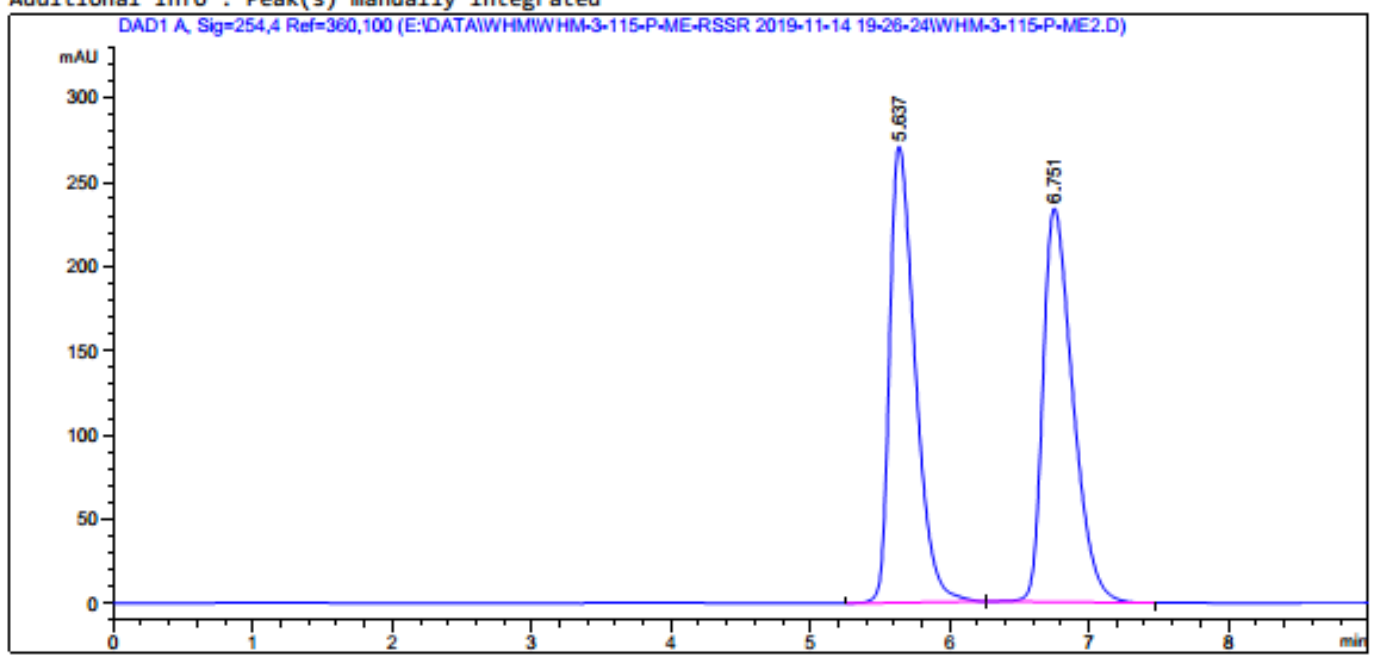

Area Percent Report

\begin{tabular}{|c|c|}
\hline Sorted By & Signal \\
\hline Multiplier & 1.0000 \\
\hline Dilution & 1.0000 \\
\hline
\end{tabular}

Do not use Multiplier \& Dilution Factor with ISTDs

Signal 1: DAD1 A, Sig $=254,4$ Ref $=360,100$

\begin{tabular}{|c|c|c|c|c|c|}
\hline $\begin{array}{c}\text { Peak } \\
\#\end{array}$ & $\begin{array}{l}\text { RetTime Type } \\
\text { [min] }\end{array}$ & $\begin{array}{l}\text { Width } \\
\text { [min] }\end{array}$ & $\begin{array}{c}\text { Area } \\
{\left[\mathrm{mAU}^{*} \mathrm{~s}\right]}\end{array}$ & $\begin{array}{l}\text { Height } \\
\text { [mAU] }\end{array}$ & $\begin{array}{c}\text { Area } \\
\text { \% }\end{array}$ \\
\hline$\ldots$ & $-\ldots$. & ........ & $|-\ldots . .2|$ & | & - \\
\hline 1 & $5.637 \mathrm{BB}$ & 0.1962 & 3441.20874 & 270.81540 & 49.7145 \\
\hline 2 & $6.751 \mathrm{BB}$ & 0.2258 & 3480.73560 & 233.63716 & 50.2855 \\
\hline Tot & : & & 6921.94434 & 584.45256 & \\
\hline
\end{tabular}

\section{*** End of Report ***}




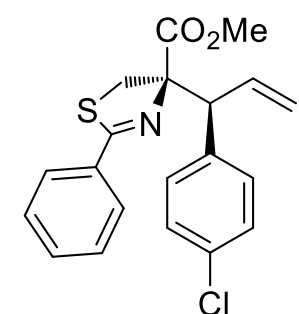

\section{$(R, S)-3$ ic}

Data file E: \DATA WWHMWHM-3-114-SZ-P-CL 2019-11-14 12-20-47\WHM-3-114-SZ-P-CL1.D Sample Name: WHM-3-114-SZ-P-CL-RS

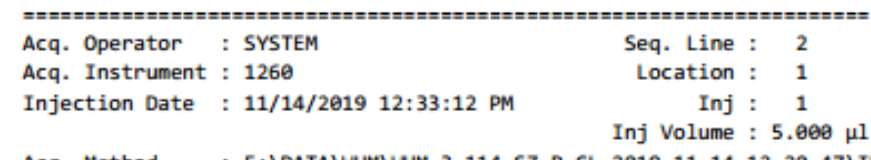

Acq. Method : E: \DATA WWHMIWHM-3-114-SZ-P-CL 2019-11-14 12-20-47\IDH-98-2--1ML-20MIN-5UL.M Last changed : 11/14/2019 12:20:47 PM by SYSTEM

Analysis Method : E: \DATA WHM WHM-3-114-SZ-P-CL 2019-11-14 12-20-47\IDH-98-2--1ML-20MIN-5UL.M (Sequence Method)

Last changed : 11/17/2019 11:27:42 AM by SYSTEM

(modified after loading)

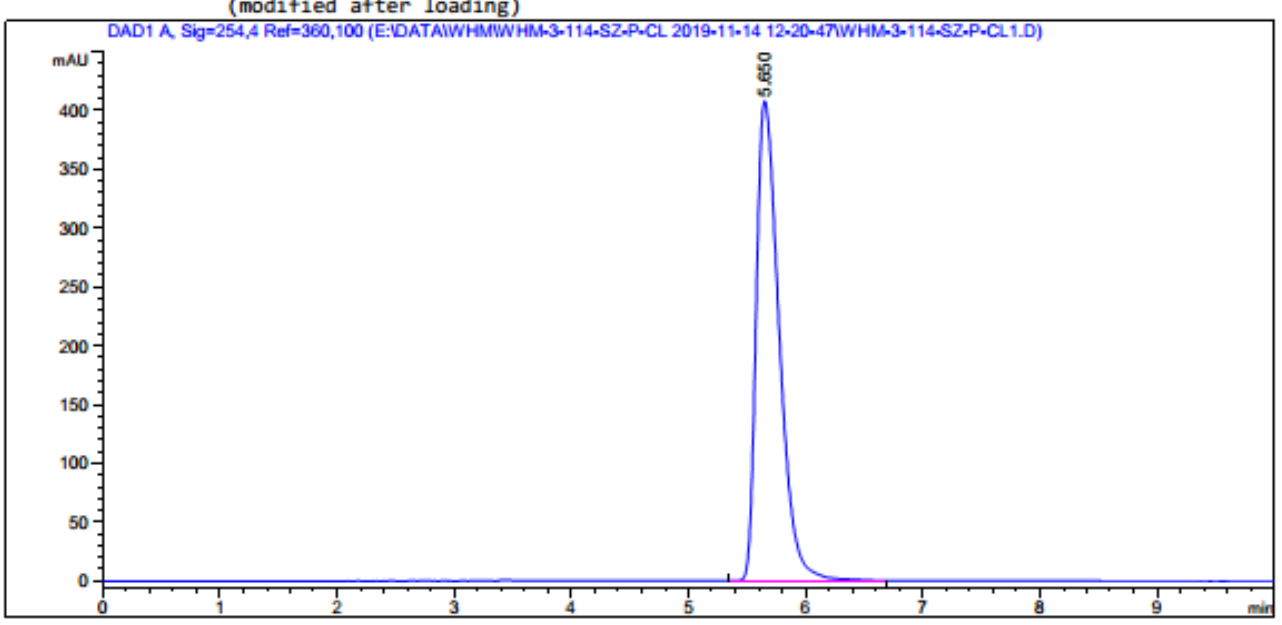

Area Percent Report

\begin{tabular}{|c|c|}
\hline Sorted By & Signal \\
\hline Multiplier & $1 . \theta 000$ \\
\hline Dilution & 1.0000 \\
\hline
\end{tabular}

Do not use Multiplier \& Dilution Factor with ISTDs

Signal 1: DAD1 A, Sig $=254,4$ Ref $=360,100$

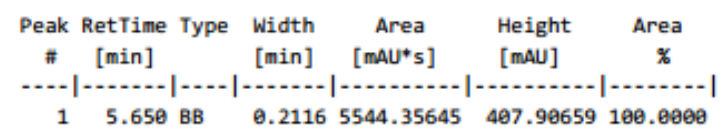

Totals : $\quad 5544.35645 \quad 467.96659$

*** End of Report *** 
<smiles>C=CC(c1cccc(Cl)c1)[C@]1(C(C)=O)CSC(c2ccccc2)=N1</smiles>

\section{racemic-3ig}

Data file E: \DATA WWM WWH-3-116-117-RACT 2019-11-16 09-39-35 WWHM-3-116-117-RAC2.D Sample Name: WHM-3-117-M-CL-RAC

\begin{tabular}{|c|c|c|c|}
\hline Acq. Operator & : SYSTEM & Seq. Line : & 3 \\
\hline Acq. Instrument & : 1260 & Location : & 2 \\
\hline Injection Date & : 11/16/2019 10:03:16 AM & $\begin{array}{r}\text { Inj : } \\
\text { Inj Volume : }\end{array}$ & $\begin{array}{l}: \quad 1 \\
: 5.900 \mu 1\end{array}$ \\
\hline
\end{tabular}

Acq. Method : E: \DATA WHM WHM-3-116-117-RACt 2019-11-16 99-39-35\IDH-98-2-5UL-10MIN-1ML.M Last changed : 11/16/2019 9:39:36 AM by SYSTEM

Analysis Method : E: \DATA WHMYWHM-3-116-117-RACt 2019-11-16 99-39-35\IDH-98-2-5UL-10MIN-1ML.M (Sequence Method)

Last changed : 11/17/2019 11:30:53 AM by SYSTEM

(modified after loading)

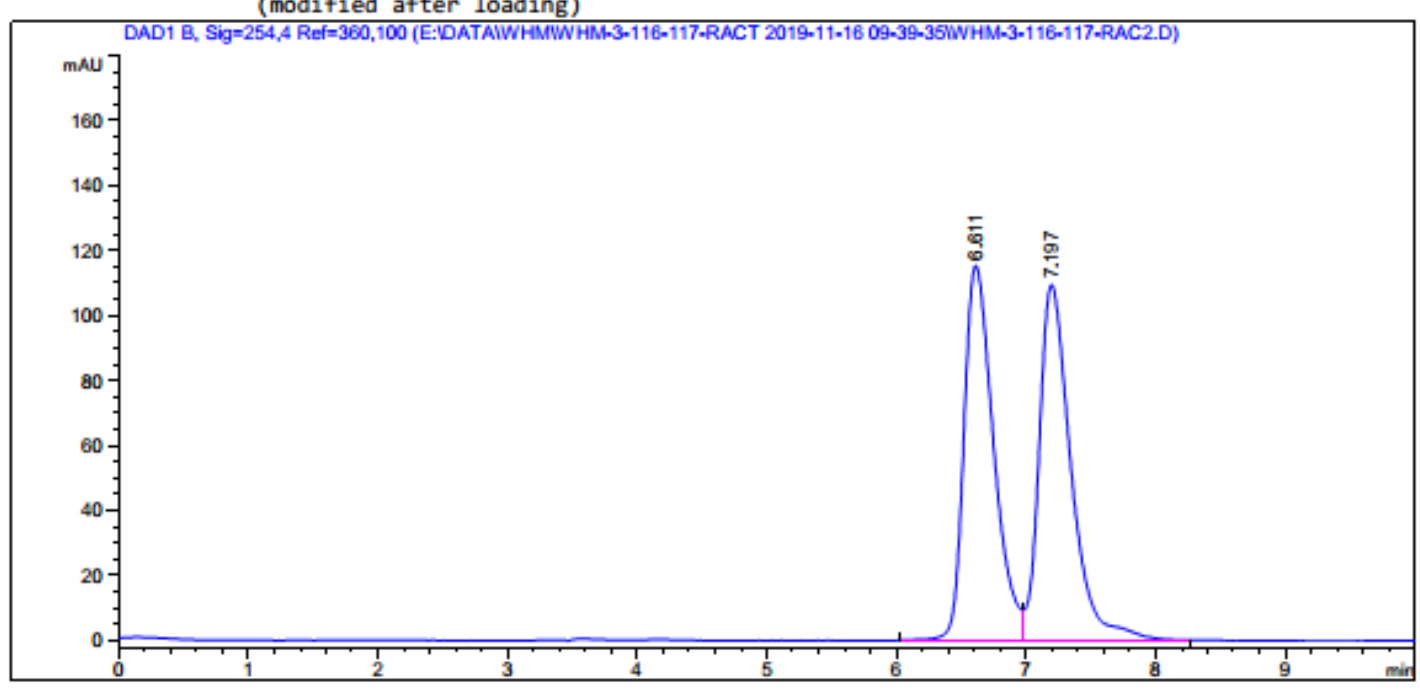

Area Percent Report

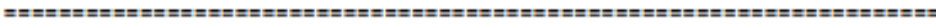

$\begin{array}{lll}\text { Sorted By } & : & \text { Signal } \\ \text { Multiplier } & : & 1.0000 \\ \text { Dilution } & : & 1.0000\end{array}$

Do not use Multiplier \& Dilution Factor with ISTDs

Signal 1: DAD1 B, Sig=254,4 Ref $=360,100$

\begin{tabular}{|c|c|c|c|c|c|c|}
\hline $\begin{array}{c}\text { Peak } \\
\#\end{array}$ & $\begin{array}{c}\text { RetTime } \\
\text { [min] }\end{array}$ & Type & $\begin{array}{l}\text { Width } \\
\text { [min] }\end{array}$ & $\begin{array}{c}\text { Area } \\
{\left[\mathrm{mAU}^{+} \mathrm{s}\right]}\end{array}$ & $\begin{array}{l}\text { Height } \\
\text { [mAU] }\end{array}$ & $\begin{array}{c}\text { Area } \\
\%\end{array}$ \\
\hline - & - & & ... & | & $\ldots$ & $\cdots$ \\
\hline 1 & 6.611 & BV & 0.2422 & 1831.33179 & 115.33579 & 49.5590 \\
\hline 2 & 7.197 & vB & 0.2566 & 1863.92712 & 109.49821 & 50.4410 \\
\hline Tot: & 5: & & & 3695.25891 & 224.83400 & \\
\hline
\end{tabular}

*** End of Report *** 
<smiles>C=CC(c1cccc(Cl)c1)[C@](C)(CS)C(C)=O</smiles>

$(R, S)-3 i g$

Data File E: \DATA \WHM \CWHM-3-116-117-RSSR 2019-11-15 12-20-16\WHM-3-116-117-RSSR3.D Sample Name: WHM-3-117-M-CL-RS
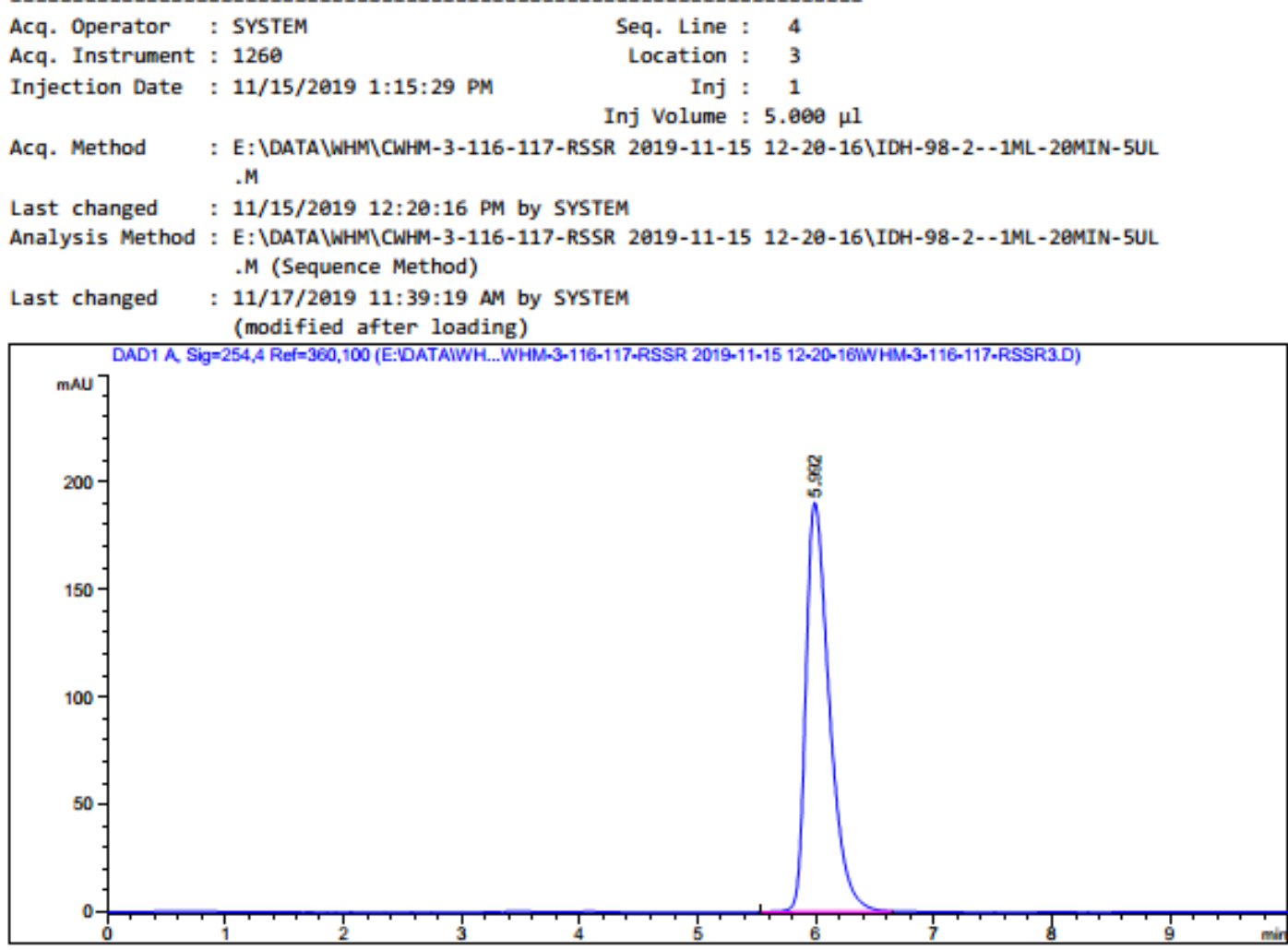

Area Percent Report

$\begin{array}{lll}\text { Sorted By } & : & \text { Signal } \\ \text { Multiplier } & : & 1.0000 \\ \text { Dilution } & : & 1.0000\end{array}$

Do not use Multiplier \& Dilution Factor with ISTDs

Signal 1: DAD1 A, Sig $=254,4$ Ref $=360,100$

\begin{tabular}{|c|c|c|c|c|c|}
\hline $\begin{array}{c}\text { Peak } \\
\#\end{array}$ & $\begin{array}{l}\text { RetTime Type } \\
\text { [min] }\end{array}$ & $\begin{array}{l}\text { Width } \\
\text { [min] }\end{array}$ & $\begin{array}{c}\text { Area } \\
{\left[\mathrm{mAU}^{*} \mathrm{~s}\right]}\end{array}$ & $\begin{array}{l}\text { Height } \\
\text { [mAU] }\end{array}$ & $\begin{array}{c}\text { Area } \\
\text { \% }\end{array}$ \\
\hline 1 & $5.992 \mathrm{BB}$ & 0.2098 & 2623.34644 & 190.30032 & 00.0000 \\
\hline
\end{tabular}

Totals : $\quad 2623.34644 \quad 190.39032$

*** End of Report *** 


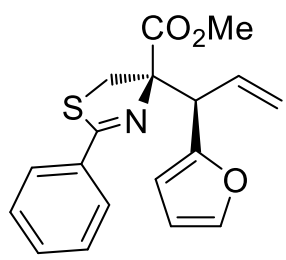

racemic-3il

Data File E: \DATA WHMYWHM-3-116-0-RAC-2 2019-11-16 15-22-19\WHM-3-116-0-RAC-21.D Sample Name: WHM-3-116-0-RAC-2

\begin{tabular}{|c|c|c|c|}
\hline Acq. Operator & : SYSTEM & Seq. Line & 2 \\
\hline Acq. Instrument & : 1260 & Location & 1 \\
\hline Injection Date & : 11/16/2019 3:34:34 PM & $\begin{array}{r}\text { Inj } \\
\text { Inj Volume }\end{array}$ & $\begin{array}{c}: \quad 1 \\
: 5.000 \mu \mathrm{l}\end{array}$ \\
\hline
\end{tabular}

Acq. Method : E: \DATA WHMIWHM-3-116-0-RAC-2 2019-11-16 15-22-19\IDH-98-2--1ML-20MIN-5UL.M Last changed : 11/16/2019 3:48:33 PM by SYSTEM (modified after loading)

Analysis Method : E: \DATA WHM\WHM-3-116-0-RAC-2 2019-11-16 15-22-19\IDH-98-2--1ML-20MIN-5UL.M (Sequence Method)

Last changed : 11/17/2019 11:34:35 AM by SYSTEM

(modified after loading)

Additional Info : Peak(s) manually integrated

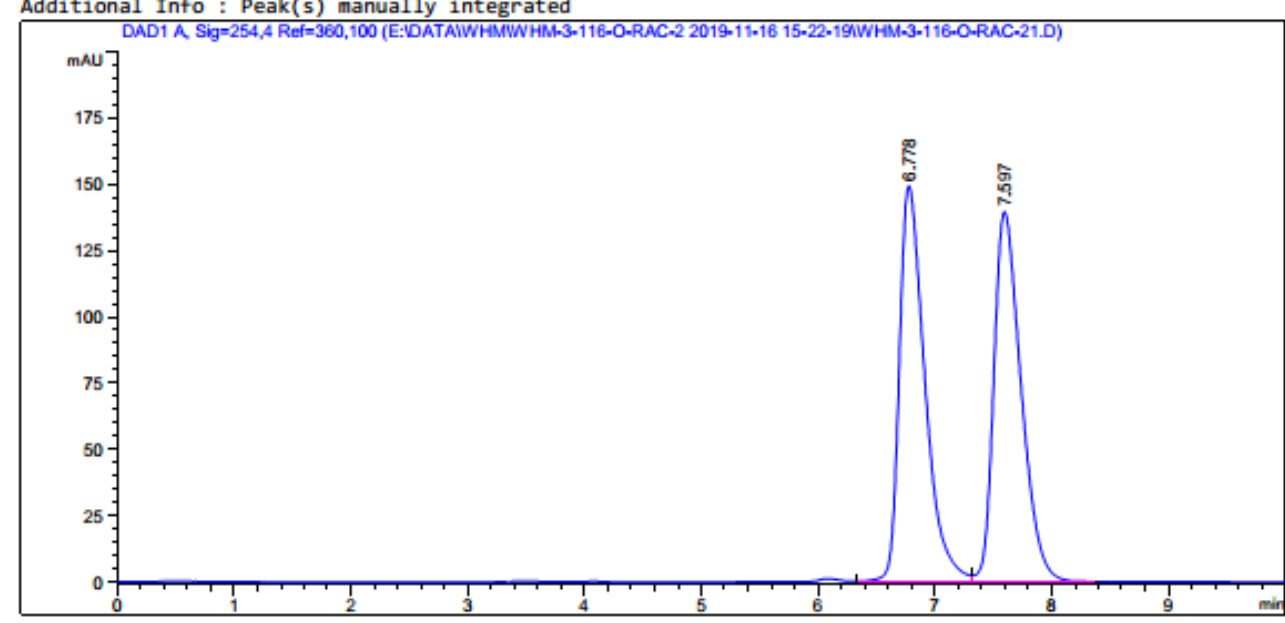

Area Percent Repor

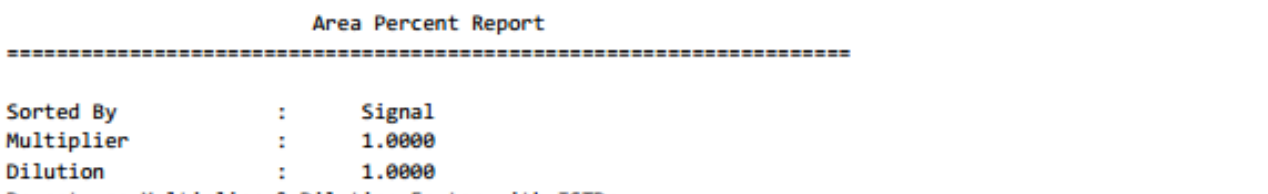

Do not use Multiplier \& Dilution Factor with ISTDs

Signal 1: DAD1 A, Sig $=254,4$ Ref $=360,100$

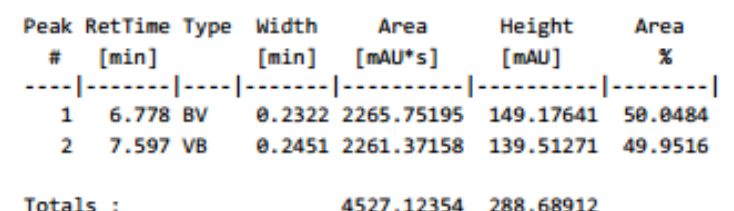

Totals : $\quad 4527.12354 \quad 288.68912$

*** End of Report *** 


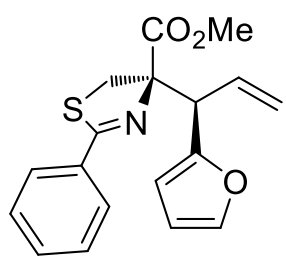

$(R, R)-3$ il

Data File E: \DATAWWHM \CWHM-3-116-117-RSSR 2019-11-15 12-20-16\WHM-3-116-117-RSSR1.D Sample Name: WHM-3-116-0-RS

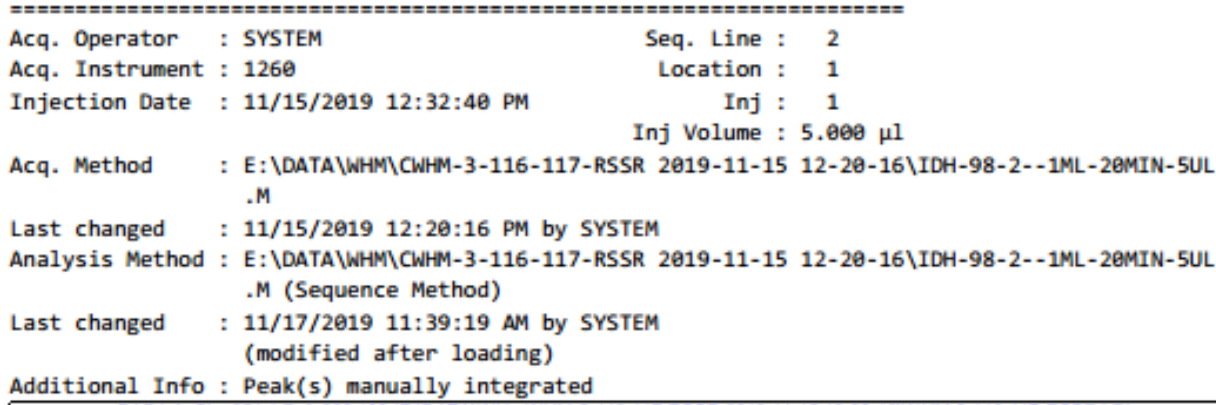

Acq. Method : E: \DATA WHM CWHM-3-116-117-RSSR 2019-11-15 12-20-16\IDH-98-2--1ML-20MIN-5UL M

Last changed : 11/15/2019 12:20:16 PM by SYSTEM

Analysis Method : E: \DATA WHMYCWHM-3-116-117-RSSR 2019-11-15 12-20-16\IDH-98-2--1ML-20MIN-5UL .M (Sequence Method)

Last changed : 11/17/2019 11:39:19 AM by SYSTEM

(modified after loading)

Additional Info: Peak(s) manually integrated

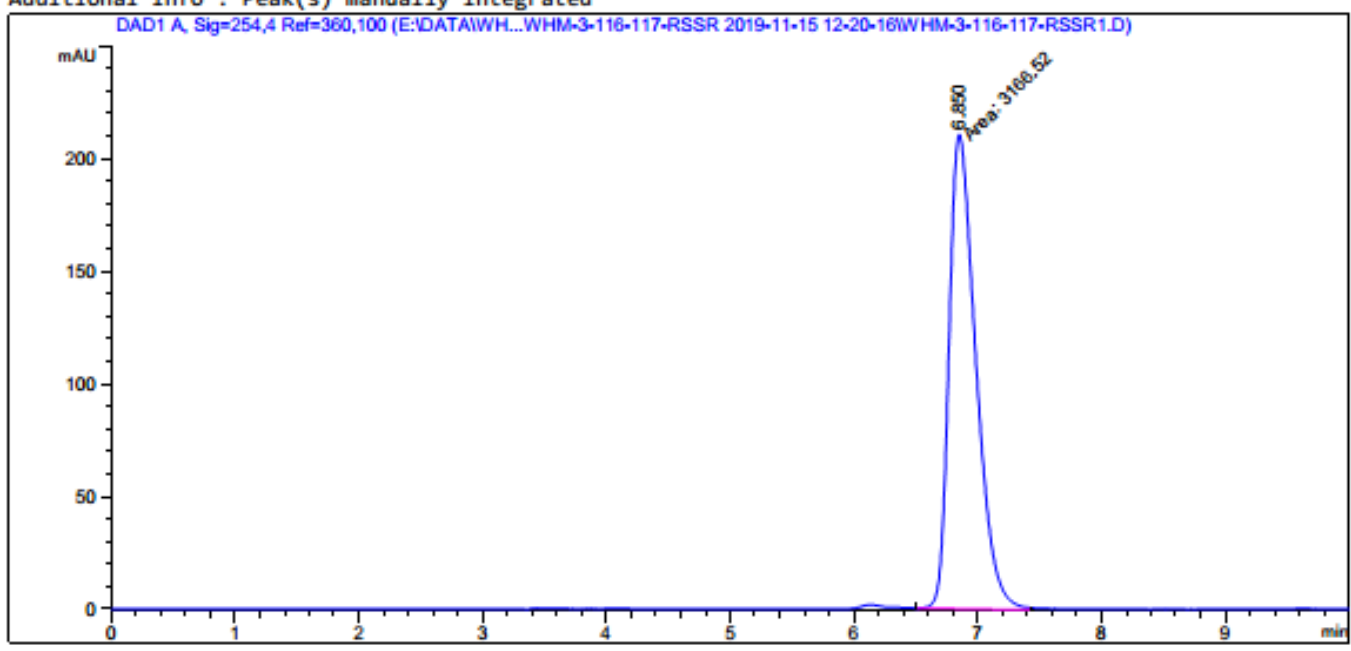

Area Percent Report

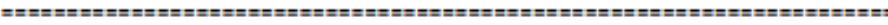

Sorted By : : Signal

Multiplier : $\quad 1.0000$

Dilution : 1.0000

Do not use Multiplier \& Dilution Factor with ISTDs

Signal 1: DAD1 A, Sig $=254,4$ Ref $=360,100$

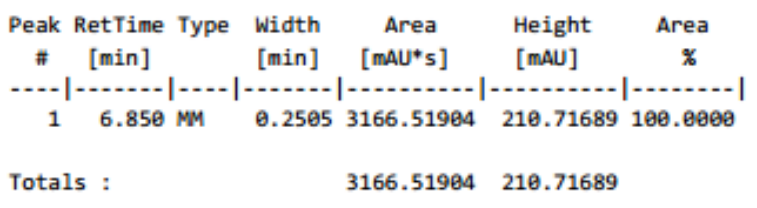

*** End of Report *** 


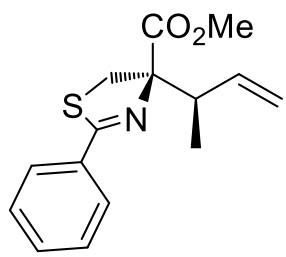

\section{racemic-3im}

Data file E: \DATA WHM WWH-3-84-ME-RSSR-3 2019-10-20 10-28-12\WHM-3-84-ME-RAC.D Sample Name: WHM-3-84-ME-RAC

\begin{tabular}{|c|c|c|c|}
\hline Acq. Operator & : SYSTEM & Seq. Line & : \\
\hline Acq. Instrument & : 1260 & Location & 11 \\
\hline Injection Date & : 10/20/2019 10:29:39 AM & $\begin{array}{r}\text { Inj } \\
\text { Inj Volume }\end{array}$ & $\begin{array}{c}1 \\
: 5.000 \mu 1\end{array}$ \\
\hline
\end{tabular}

Acq. Method : E: \DATA WHMYWHM-3-84-ME-RSSR-3 2019-10-20 10-28-12\O0-H-99-1--1ML-5ul-10min -DADALL.M

Last changed : 10/20/2019 10:28:12 AM by SYSTEM

Analysis Method : E: \DATA WHM WHM-3-84-ME-RSSR-3 2019-10-20 10-28-12\OO-H-99-1--1ML-5ul-10min -DADALL.M (Sequence Method)

Last changed : 10/23/2019 8:56:55 PM by SYSTEM

(modified after loading)

Additional Info : Peak(s) manually integrated

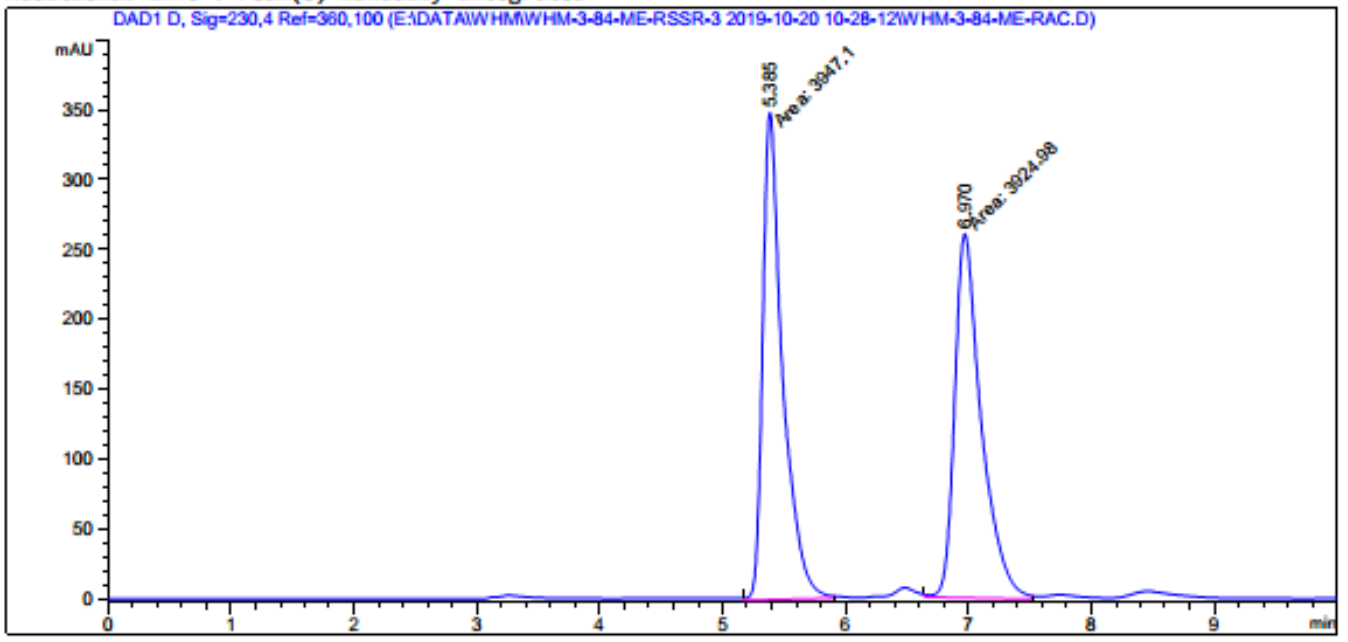

Area Percent Report

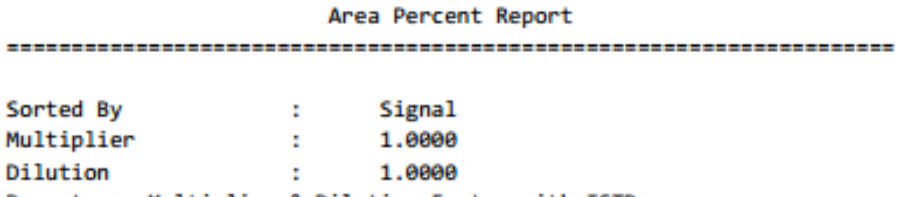

Do not use Multiplier \& Dilution Factor with ISTDs

Signal 1: DAD1 D, Sig=230,4 Ref=360,100

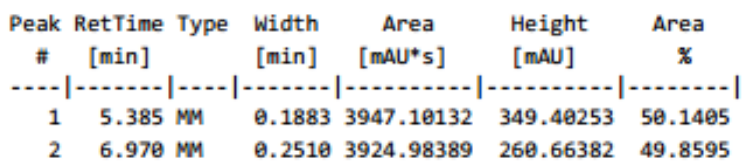

Totals : $\quad 7872.08521610 .06635$

*** End of Report *** 


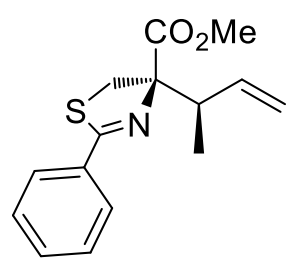

$(R, R)$-3im

Data file E: \DATA WHM WWHM-3-84-ME-RSSR-3 2019-10-20 08-41-35 \WHM-3-84-ME-RSSR-31.D Sample Name: WHM-3-84-ME-RS-3

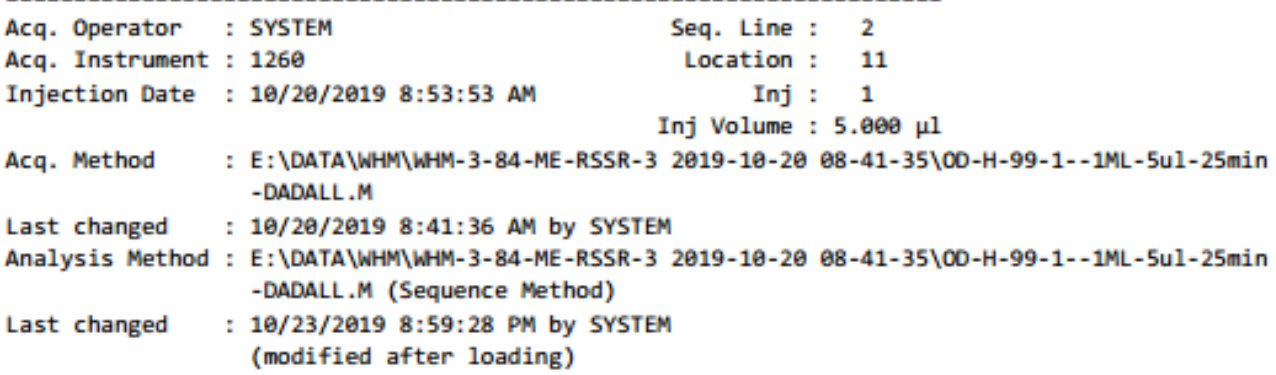

Acq. Method : E: \DATA WHMYWHM-3-84-ME-RSSR-3 2019-10-20 98-41-35 -DADALL.M

Last changed : 10/20/2019 8:41:36 AM by SYSTEM

Analysis Method : E: \DATA \WHM\WHM-3-84-ME-RSSR-3 2019-10-20 08-41-35\O0-H-99-1--1ML-5ul-25min DADALL.M (Sequence Method)

Last changed : 10/23/2019 8:59:28 PM by SYSTEM (modified after loading)

Additional Info: Peak(s) manually integrated
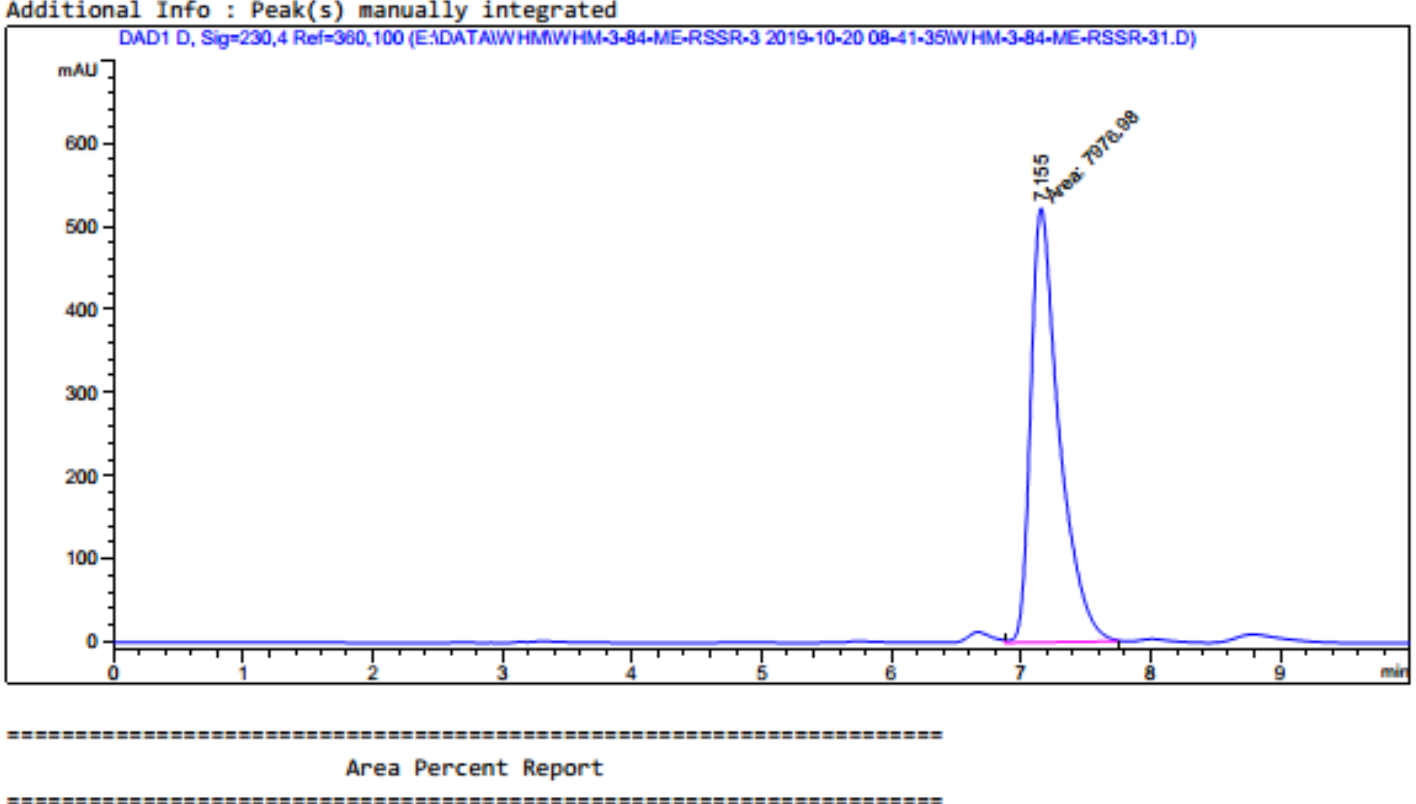

$\begin{array}{lll}\text { Sorted By } & : & \text { Signal } \\ \text { Multiplier } & : & 1.0000 \\ \text { Dilution } & : & 1.0000\end{array}$

Do not use Multiplier \& Dilution Factor with ISTDs

Signal 1: DAD1 D, Sig $=230,4$ Ref $=360,100$

$$
\begin{aligned}
& \text { Peak RetTime Type Width Area Height Area } \\
& \text { \# [min] [min] [mAU*s] [mAU] }
\end{aligned}
$$

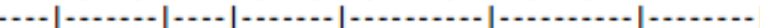

$$
\begin{aligned}
& \begin{array}{llllll}
1 & 7.155 \mathrm{MM} & 0.2544 & 7976.97949 & 522.50623 & 100.0009
\end{array} \\
& \text { Totals : } \quad 7976.97949 \quad 522.50623
\end{aligned}
$$

$$
\text { *** End of Report *** }
$$


<smiles>COC(=O)C1(C(c2ccccc2)C2CC2)COC(c2ccc(C(F)(F)F)cc2)N=C1C(C)(C)C</smiles>

Data file E: \DATA WHM WHM-3-103-111-112 2019-11-13 99-11-00\WHM-3-103-HBW-RAC-111-1121.D Sample Name: WHM-3-103-HBW-RAC

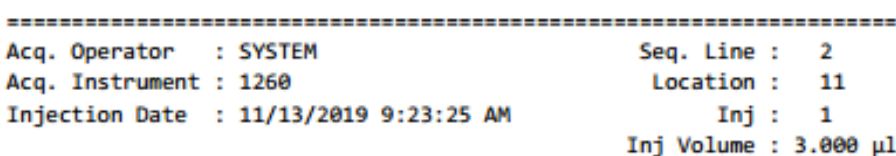

Inj Volume : $3.000 \mu \mathrm{l}$

Acq. Method : E: \DATA WHMYWHM-3-103-111-112 2019-11-13 99-11-e日\IDH-95-5--3UL-15MIN1. eML. $M$

Last changed : 11/13/2019 9:11:00 AM by SYSTEM

Analysis Method : E: \DATA WHM WHM-3-103-111-112 2019-11-13 09-11-00\IDH-95-5--3UL-15MIN1. eML.

$M$ (Sequence Method)

Last changed : 11/13/2019 9:52:40 PM by SYSTEM (modified after loading)
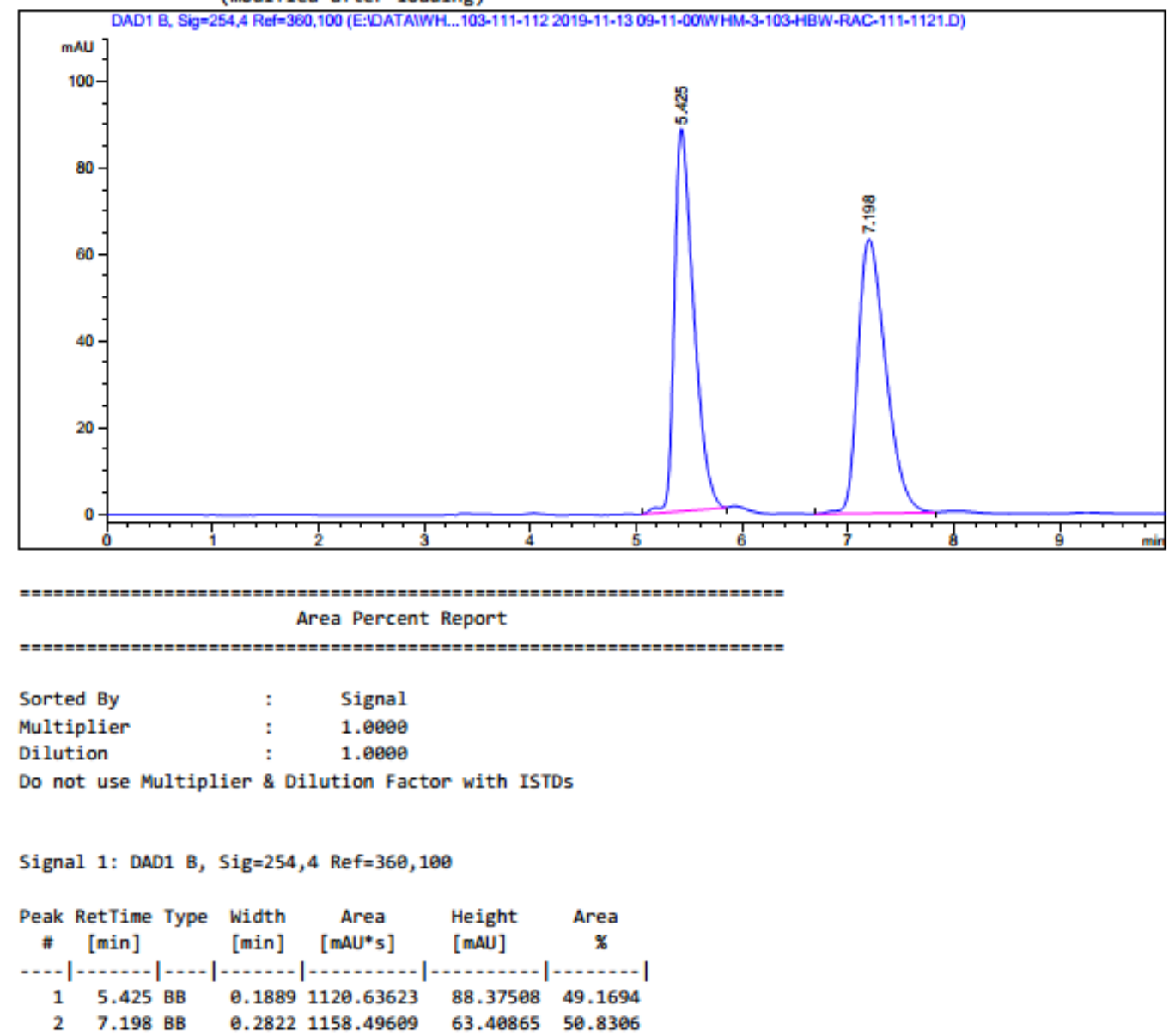

Totals : $\quad 2279.13232 \quad 151.78372$

\section{*** End of Report ***}


<smiles>COC(=O)[C@]1(C(c2ccccc2)C2CC2)COC(c2ccc(C(F)(F)F)cc2)=N1</smiles>

Data file E: \DATA WHM WWH-3-87-103-RSSR-HBW 2019-11-11 20-20-13\WHM-3-87-103-HBW-RSSR1.D Sample Name: WHM--3-87-RS-HBW

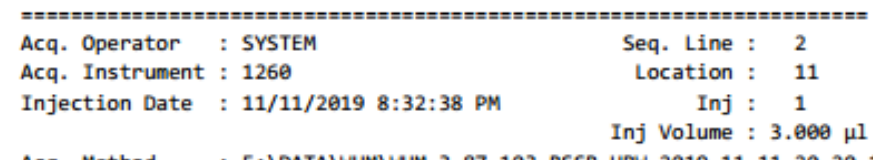

Acq. Method : E: \DATA WWHMWHM-3-87-103-RSSR-HBW 2019-11-11 20-20-13\IDH-95-5--3UL-15MIN1. OML.M

Last changed : 11/11/2019 $8: 20: 13$ PM by SYSTEM

Analysis Method : E: \DATA WHMTWHM-3-87-103-RSSR-HBW 2019-11-11 20-20-13\IDH-95-5--3UL-15MIN1. OML.M (Sequence Method)

Last changed : 11/13/2019 9:56:54 PM by SYSTEM (modified after loading)

Additional Info : Peak(s) manually integrated
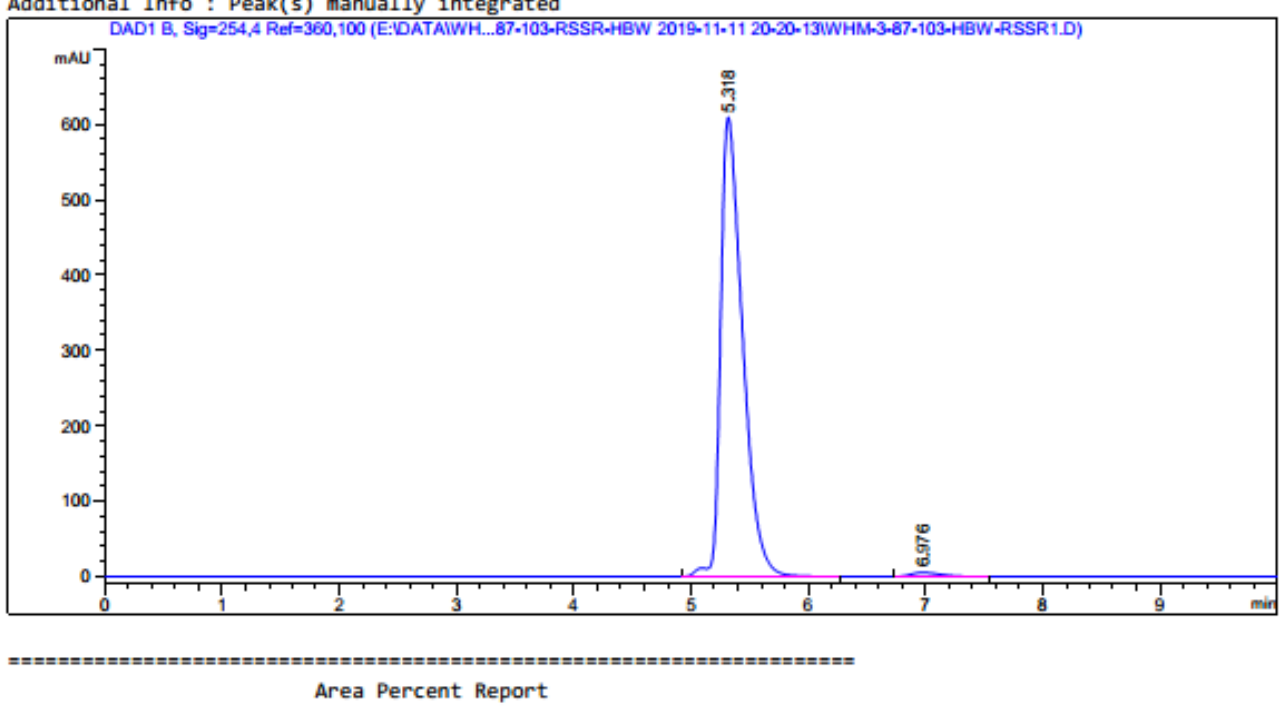

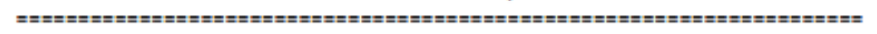

$\begin{array}{lcc}\text { Sorted By } & : & \text { Signal } \\ \text { Multiplier } & : & 1.000 \ominus \\ \text { Dilution } & : & 1.0000 \\ \text { Do not use Multiplier } & \& & \text { Dilution Factor with ISTDs }\end{array}$

Signal 1: DAD1 B, Sig $=254,4$ Ref $=360,100$

\begin{tabular}{|c|c|c|c|c|c|c|}
\hline $\begin{array}{c}\text { Peak } \\
\#\end{array}$ & $\begin{array}{l}\text { RetTime } \\
\text { [min] }\end{array}$ & Type & $\begin{array}{l}\text { Width } \\
\text { [min] }\end{array}$ & $\begin{array}{c}\text { Area } \\
{\left[\mathrm{mAU} \mathbf{u}^{*} \mathrm{~s}\right]}\end{array}$ & $\begin{array}{l}\text { Height } \\
\text { [mAU] }\end{array}$ & $\begin{array}{c}\text { Area } \\
\text { \% }\end{array}$ \\
\hline 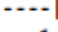 & & & & 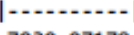 & 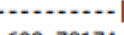 & . \\
\hline 1 & 5.318 & VB R & 0.1922 & 7930.07178 & 609.78174 & 98.8824 \\
\hline 2 & 6.976 & BB & 0.2487 & 89.62975 & 5.68449 & 1.1176 \\
\hline
\end{tabular}

Totals :

$8019.70152 \quad 614.86623$

\section{*** End of Report ***}

